\title{
Versuche über den Einfluss der Rissbildung auf die dynamischen Eigenschaften von Leichtbeton- und Betonbalken
}

\section{Working Paper}

Author(s):

Bachmann, Hugo; Dieterle, Rudolf

Publication date:

1979

Permanent link:

https://doi.org/10.3929/ethz-a-000187922

Rights / license:

In Copyright - Non-Commercial Use Permitted

Originally published in:

Bericht / Institut für Baustatik und Konstruktion ETH Zürich 7501(1) 
Versuche über den Einfluss der Rissbildung auf die dynamischen Eigenschaften von Leichtbetonund Betonbalken

Rudolf Dieterle

Hugo Bachmann 
CIP-Kurztitelaufnahme der Deutschen Bibliothek

\section{Dieterle, Rudolf:}

Versuche über den Einfluss der Rissbildung auf die dynamischen Eigenschaften von Leichtbetonund Betonbalken / Rudolf Dieterle ; Hugo Bachmann. - Basel, Boston, Stuttgart : Birkhäuser, 1979.

(Bericht / Institut für Baustatik und Konstruktion ETH Zürich ; Nr. 7501-1)

ISBN 3-7643-1163-0

NE: Bachmann, Hugo:

Nachdruck verboten.

Alle Rechte, insbesondere das der Übersetzuna in fremde Sprachen und der Reproduktion auf photostatischem Wege oder durch Mikrofilm,

vorbehalten.

(C) Birkhäuser Verlag Basel, 1979

ISBN 3-7643-1163-0 


\title{
Versuche über den Einfluss der Rissbildung auf die dynamischen Eigenschaften von Leichtbeton- und Betonbalken
}

\author{
von \\ Dipl. Ing. Rudolf Dieterle \\ Prof. Dr. Hugo Bachmann \\ Institut für Baustatik und Konstruktion \\ Eidgenössische Technische Hochschule Zürich
}


1. EINLEITUNG

1.1 Allgemeines

1.2 Zielsetzung

2. THEORETISCHE GRUNDLAGEN 2

2.1 Allgemeines 2

2.2 Uebęrblick über die Dämpfung 4

2.2 .1 Dämpfungsarten 4

2.2.2 Ursachen der Dämpfung 4

2.2.3 Modelle zur Berücksichtigung der Dämpfung 5

2.2.4 Möglichkeiten zur experimentellen Bestimmung der Dämpfung 7

2.2.5 Umrechnung der Dämpfungskenngrössen 11

2.3 Frühere Versuche zur Bestimmung der Dämpfungseigenschaften von
Stahlbeton- und Spannbeton-Bauteilen

3. VERSUCHSBALKEN 12

3.1 Versuchsplanung $\quad 12$

3.1.1 Statisches System und wichtigste Abmessungen 12

3.1 .2 Armierungen 13

3.1 .3 Betonarten 13

3.1 .4 Zusatzmassen 13

3.1.5 Prüfarten 14

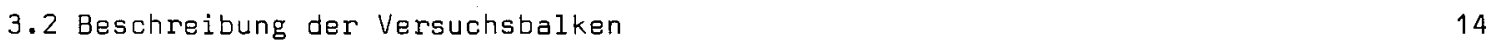

3.2.1 Allgemeines $\quad 14$

3.2.2 Armierungsstahl 14

3.2.3 Leichtbeton und Beton 15

3.2.4 Rechnerische Werte 16

4. VERSUCHSDLRCHFUEHRUNG 17

$\begin{array}{ll}4.1 \text { Versuchsanlage } & 17\end{array}$

4.1.1 Ausschwingvorrichtung 17

$\begin{array}{ll}4.1 .2 & \text { Schwingungserreger }\end{array}$

4.1.3 Statische Belastungsvorrichtung 18

$\begin{array}{ll}4.1 .4 \text { Zusatzmassen } & 18\end{array}$

4.1 .5 Lager $\quad 18$

4.2 Versuchsablauf $\quad 19$

4.3 Messungen $\quad 21$

4.4 Auswertungen $\quad 22$

4.4.1 Auswertung von Hand $\quad 22$

4.4.2 Auswertung durch Prozessrechner 23

5. VERSUCHSRESULTATE 25

5.1 Durchbiegungen und Steifigkeiten $\quad 25$

5.1 .1 Bei statischer Belastung 26

5.1.2 Bei dynamischer Belastung 28

$\begin{array}{ll}5.2 \text { Beschleunigungen } & 30\end{array}$

5.3 Biegemomente und Querkräfte 31

$\begin{array}{ll}5.4 \text { Dehnungen } & 31\end{array}$

$\begin{array}{ll}5.5 \text { Rissverhalten } & 33\end{array}$

$\begin{array}{lr}5.6 \text { Eigenfrequenzen } & 34\end{array}$ 
Seite

5.7 Dämpfung 35

5.7.1 Bestimmung aus Ausschwingversuchen 35

5.7.2 Bestimmung aus Resonanzversuchen 36

5.7.3 Wesentliche Erkenntnisse 36

ZUSAMMENFASSUNG

$\begin{array}{ll}\text { RESUME } & 41\end{array}$

$\begin{array}{lr}\text { SUMMARY } & 44\end{array}$

$\begin{array}{lr}\text { VERDANKUNGEN } & 47\end{array}$

BEZEICHNUNGEN

$\begin{array}{ll}\text { LITERATURVERZEICHNIS } & 51\end{array}$

TABELLEN UND BILDER

\begin{tabular}{ll} 
ANHANG & A1 \\
\hline &
\end{tabular} 


\subsection{Allgemeines}

Die Technik der dynamischen Analyse von Tragwerken mittels Computerprogrammen ist heute weit fortgeschritten, und es besteht darüber eine umfangreiche Literatur. Die Ergebnisse können jedoch nur brauchbar sein, wenn sie auf wirklichkeitsnahen Grundlagen beruhen. Solche Grundlagen fehlen heute auf dem Gebiet des Stahlleichtbetons noch weitgeheñd, für den Stahlbeton sind sie noch sehr unvollständig. Insbesondere fehlen sowahl für Leichtbeton- als auch für Betonkonstruktionen grundlegende Kenntnisse über den Einfluss der Rissbildung, der Beanspruchungshöhe, des Armierungsgehaltes, des Vorspanngrades und der . Betonart auf Bauwerkseigenschaften wie zu erwartende Eigenfrequenz, voraussichtliches Dämpfungsverhalten sowie Grösse der Amplituden bei erzwungenen Schwingungen.

Leichtbeton weist gegenüber Beton ein geringeres Raumgewicht und einen wesentlich kleineren E-Modul auf, erreicht jedoch ungefähr dieselbe Festigkeit. Durch Verwendung von Leichtbeton können deshalb Bauwerke mit gegenüber Beton grösserer Schlankheit wirtschaftlich erstellt werden. Mit zunehmender Schlankheit steigt jedoch die Anfälligkeit für dynamische Krafteinwirkungen.

Bis heute sind noch keine systematischen Untersuchungen zum Vergleich des dynamischen Verhaltens von Leichtbetankonstruktionen mit demjenigen analog ausgebildeter Konstruktionen aus normalem Beton bekannt geworden. Ein solcher Vergleich kann jedoch für die Baustoffwahl von wesentlicher Bedeutung sein.

Zur Klärung der aufgeführten Fragen sind am Institut für Baustatik und Konstruktion an der ETH Zürich insgesamt sieben schlaff armierte Leichtbeton- und Betonbalken in dynamischen Versuchen geprüft warden. Die drei Leichtbetonbalken mit Leichtzuschlagstoffen Leca hade unterschieden sich in der Grässe der Längsarmierung. Die drei Betonbalken wurden als Duplikate der Leichtbetonbalken ausgebildet. Damit konnte ein direkter Vergleich zwischen analogen Balken aus Leichtbeton und Beton durchgeführt werden. Zusätzlich wurde ein vierter Leichtbetonbalken mit Leichtzuschlagstoffen Leca geprüft.

\subsection{Zielsetzung}

Die vorliegende Untersuchung dient der Erforschung dès Einflusses

- der Rissbildung

- der Beanspruchungshöhe

- des Armierungsgehaltes

auf die dynamischen Eigenschaften von schlaff armierten Leichtbeton- und Betonbalken, insbesondere auf

- die Steifigkeit resp. die Eigenfrequenz und

- das Dämpfungsverhalten.

Dem Vergleich analoger Leichtbeton- und Betonbalken war besondere Aufmerksamkeit zu schenken. Die Versuchsresultate sollten als Grundlage dienen für die Entwicklung theoretischer Modelle zur wirklichkeitsnahen Erfassung des dynamischen Verhaltens von Leichtbeton- und Betonkonstruktionen. 
In diesem Kapitel wird versucht, die Grundlagen zum besseren Verständnis der durchgeführten Versuche bereitzustellen. Nach einer kurzen Darstellung der für eine dynamische Berechnung benötigten Schwingungsdifferentialgleichungen wird ausführlicher auf die Dämp fung eingegangen. Am Schluss werden die wesentlichen Erkenntnisse aus der Auswertung von andernorts erfolgten Versuchen über das Dämpfungsverhalten von Stahlbetonbalken wiedergegeben.

\subsection{Allgeme ines}

Dynamische Beanspruchungen von Bauteilen und Tragwerken können entstehen durch

- Wind

- Erdbeben

- Wasserwellen

- Explosionen

- laufende Maschinen

- etc.

Diese periodischen, nicht periodischen oder stochastischen Belastungen bewirken beim Tragwerk mit der Zeit veränderliche Verformungen, Geschwindigkeiten und Beschleunigungen. Letztere sind die Ursache von Trägheitskräften, die zusätzlich zur statischen Belastung auf das Tragwerk einwirken. Je nach deren Grösse können die dynamischen Zusatzbeanspruchungen für die Bemessung eines Tragwerks massgebend werden.

Tragwerke sind Schwingsysteme mit - theoretisch gesehen - unendlich vielen Freiheits graden. Dem entsprechen Differentialgleichungssysteme mit unendlich vielen Gleichungen. In der Regel kann jedoch die Zahl der relevanten Freiheitsgrade beschränkt und damit der Lösungsaufwand in erträglichem Rahmen gehalten werden.

Wendet man das Prinzip von d'Alembert auf den einfachsten Schwingertyp, den Einmassenschwinger, an, so erhält man die Differentialgleichung

$$
m \cdot \ddot{w}+R(z, \dot{z})=P(t)
$$

Die Trägheitskraft $m \bullet \ddot{w}$ und die bewegungshemmende Kraft $R(z, \dot{z})$ müssen also zu jedem Zeitpunkt mit der äusseren Belastung $P(t)$ im Gleichgewicht sein.

Durch die Belastung $P(t)$ wird dem Tragwerk resp. Schwingsystem Energie zugeführt. Kann diese Energie nicht vom Tragwerk absorbiert werden (z.B. durch Umwandlung in Wärme), so wird das Tragwerk immer weiter aufgeschaukelt bis es zu Bruch geht. Bekanntlich besitzt jedoch jedes Material die Fähigkeit, allerdings in sehr unterschiedlichem Ausmass, Bewegungsenergie zu absorbieren. Diese Eigenschaft wird Dämpfungsvermögen genannt. Die Grösse der Dämpfung eines Materials bzw. einer Konstruktion hat einen grossen Einfluss auf die auftretenden Beschleunigungen und Trägheitskräfte.

Man unterscheidet zwischen linearen und nichtlinearen Schwingern resp. Differentialgleichungen.

Bei linearen Einmassenschwingern resp. Differentialgleichungen wird $R$ aufgeteilt in einen Teil $k \cdot z$, der nur zu $z$ und einen Teil $c \cdot \dot{z}$, der nur zu $\dot{z}$ proportional ist ( $k$, $c=k o n-$ stant). Wird zugleich angenommen, dass die absolute Verschiebung $w$ und die relative Verschiebung $z$ gleich sind (ruhender Aufhängepunkt) so erhält man die Bewegungsgleichung 
eines Einmassenschwingers mit viskoser Dämpfung

$$
m \cdot \ddot{z}+c \cdot \dot{z}+k \cdot z=P(t)
$$

In Bild 1 ist das Modell des zur Gl. (2) gehörenden Einmassenschwingers abgebildet: $k \cdot z$ entspricht der elastischen Rückstellkraft der Feder, c·ż der Dämpfungskraft des im Modell angegebenen Dämpfungselementes. Damit $c$ und $k$ als konstant betrachtet werden dürfen, müssen sie unabhängig von der Geschichte der Bewegung, der Amplitude, der Belastung und der Zeit sein. Ungedämpfte Systeme $(c=0)$ sind konservativ. Sobald die Dämpfung in die Betrachtung miteinbezogen wird, haben wir es mit einem nichtkonservativen System zu tun, da Energie dissipiert wird.

Bei linearen Mehrmassenschwingein mit $n$ Freiheitsgraden müssen $n$ Differentialgleichungen mit zu Gl. (2) analogem Aufbau aufgestellt werden. Benützt man die Matrizen-Schreibweise, so führt dies zum Differentialgleichungssystem

$$
[M]\{\ddot{z}\}+[C]\{\dot{z}\}+[K]\{z\}=\{P(t)\}
$$

Für [C] wird häufig ein steifigkeitsproportionaler, ein massenproportionaler oder ein kombinierter steifigkeits-massenproportionaler Ansatz gemacht:

$$
\begin{aligned}
& {[C]=\alpha[K]} \\
& {[C]=\beta[M]} \\
& {[C]=\alpha[K]+\beta[M]}
\end{aligned}
$$

Darin sind $\alpha$ und $\beta$ zu wählende Konstanten. Die obigen Ansätze (4) werden proportional genannt. Mit diesen speziellen Ansätzen für die Dämpfungsmatrix können die Differentialgleichungssysteme (3) durch Entkoppelung der einzelnen Differentialgleichungen vereinfacht gelöst werden.

Bei proportionaler Dämpfung schwingt, das System in einer Kombination von verschiedenen Eigenformen, welche die gleiche Form wie beim ungedämpften System haben, und deren Amplituden im Laufe der Zeit über das ganze System gleichmässig abnehmen. Die Schwingungsknoten bleiben stets am gleichen Ort.

Bei nichtlinearen Einmassenschwingern ergeben sich nichtlineare Differentialgleichungen. Nichtlinearität kann durch ein nichtlineares Kraft-Verschiebungs-Gesetz (Federgesetz), d.h. durch

- $k \neq$ konstant und/ader

- eine Abweichung vom verschiebungsproportionalen Ansatz

und/oder durch ein nichtlineares Dämpfungsgesetz, d.h. durch

- c $\neq$ konstant und/oder

- eine Abweichung vom geschwindigkeitsproportionalen Ansatz

bedingt sein.

Bei nichtlinearen Mehrmassenschwingern können die nichtlinearen Differentialgleichungssysteme nicht entkoppelt werden. Die Lösung erfolgt über Näherungsverfahren (Verfahren der Harmonischen Balance, Verfahren von Krylow/Bogoljubow, etc.) oder über numerische Methoden.

Je nach der Modell-Vorstellung, die der Dämpfung zugrunde gelegt wird, muss für das 
Dämpfungsglied in den Gieichungen (2) und (3) ein entsprechender linearer oder nichtlinearer Ansatz gemacht werden.

2.2 Ueberblick über die Dämpfung

In diesem Abschnitt wird ein Ueberblick über die Dämpfungsphänomene gegeben, und anschliessend werden einige Modelle zur Erfassung der Dämpfung beschrieben.

Unter der Dämpfung eines mechanischen Schwingers versteht man den Vorgang der nicht umkehrbaren Umwandlung potentieller und kinetischer Energie in andere Energieformen, vornehmlich in Wärme [6].

Die Dämpfung entspricht dem Betrag an mechanischer Energie, der einem schwingenden System bei jedem Schwingungszyklus entzogen wird [5]. Das Vorhandensein der Dämpfung verursacht die Dissipation von Energie, so dass die Schwingungsamplituden reduziert werden, und die Bewegung zum Erliegen kommt, wenn die ganze ursprünglich gespeicherte Energie dissipiert ist [3].

\section{$\underline{2.2 .1 \text { Dämpfungsarten }}$}

Die Dämpfung mechanischer Systeme lässt sich anhand des in Bild 2 angegebenen Schemas klassieren.

Ueblicherweise wird zwischen innerer und äusserer Dämpfung unterschieden, je nachdem, ob die irreversible Energieumwandlung an den Systemgrenzen oder im Innern des Systems erfolgt. Dämpfungskräfte können sowohl vom schwingenden system selbst als auch von den umgebenden Elementen verursacht werden.

Unter Materialdämpfung versteht man die Energiedissipation innerhalb eines Kontinuums. Dagegen erfasst die Strukturdämpfung die Dämpfungserscheinungen, die zwischen den Grenzflächen von unterscheidbaren Partikeln des betreffenden Stoffes auftreten.

Unter mechanischer Dämpfung werden Dämpfungserscheinungen an den Grenzen eines Kontinuums verstanden, die z.B. durch Lagerreibung, Reibung an Systemgrenzen oder auch durch künstliche Dämpfungsvorrichtungen hervorgerufen werden.

Die Zuordnung bestimmter Dämpfungserscheinungen hängt häufig von dèr Definition und Abgrenzung des betrachteten Systems ab. Zum Beispiel kann die Dämpfung an den Korngrenzen der Zuschlagstoffe eines Betons oder an den Kontaktflächen zwischen Beton und Armierung als innere oder äussere bzw. als Materialdämpfung oder als Strukturdämpfung bezeichnet. werden.

Aus gemessenen. Dämpfungswerten lässt sich der Charakter der Energiedissipation oft nicht erkennen. Dies hat seine Ursache darin, dass die unmittelbare Folge der diesbezüglichen inneren Vorgänge im Material, nämlich der Verlust an Schwingungsenergie, nur als pauschale, makroskopische Erscheinung auftritt. Es ist zu erwarten, dass beim Beton beide Hauptarten der inneren Dämpfung, d.h. sowohl Struktur- als auch Materialdämpfung vorkommen.

\subsubsection{Ursachen der Dämpfung}

Ueber die Ursachen der Dämpfung und die zugehörigen Mechanismen ist bis jetzt sehr wenig bekannt.

Nach [5] und [6] können vermutlich besonders die folgenden Phänomene massgebend sein: 
- Flüssige Reibung (Materialdämpfung):

Der Anteil der flüssigen Reibung an der inneren Dämpfung bei Beton-Bauteilen und -Konstruktionen dürfte bedeutend sein. Das in den Gel-Partikeln des Zementes absorbierte Wasser dissipiert Schwingungsenergie durch molekulare Diffusion.

- Plastifizierungseffekte (Materialdämpfung):

Auch im sog. elastischen Beanspruchungsbereich eines als homogen aufgefassten Materials sind irreversible Verformungen auf mikroskopischer Ebene möglich (z.B. infolge von Spannungskonzentrationen an Berührungsstellen von Partikeln). Der Anteil der Plastifizierungseffekte an der gesamten Materialdämpfung dürfte jedoch nur bei hohen Beanspruchungen nennenswert sein.

\section{- Thermoelastische Effekte (Materialdämpfung):}

Bei einem schwingenden Biegeträger werden die jeweiligen Zugfasern abgekühlt, die Druckfasern erwärmt, so dass innerhalb des Querschnittes ein Wärmeausgleich vor sich gehen will. Bei periodisch wechselnden Spannungen entsteht ein periodisch wechselnder Temperaturgradient. Bei sehr hohen Frequenzen bleibt keine Zeit für einen Wärmefluss (adiabatische Verhältnisse), bei sehr niedrigen frequenzen wird das Wärmegleichgewicht ständig aufrecht erhalten (isotherme Verhältnisse). In beiden Extremfällen entsteht kein Energieverlust, während bei mittleren Frequenzen Energie dissipiert wird.

- Trockene Reibung (Strukturdämpfung):

Trockene Reibung oder Coulomb-Reibung kann durch Gleitbewegungen zwischen benachbarten Partikeln entstehen. Der Anteil der trockenen Reibung an der Gesamtdämpfung ist unter anderem vom Spannungszustand abhängig.

- Rissbildung an Haftflächen (Strukturdämpfung):

Beim Bruch zwischen Gruppen von benachbarten Partikeln kann Schwingungsenergie dissipiert werden.

\subsubsection{Modelle zur Berücksichtigung der Dämpfung}

Aufgrund der verschiedenen Vorstellungen über die Ursachen der Dämpfung resultieren verschiedene Modelle zur rechnerischen Berücksichtigung der Dämpfungseigenschaften eines Schwingers.

\section{a) Viskose Dämpfung (Flüssige Reibung)}

Durch das Modell der viskosen Dämpfung lässt sich insbesondere die flüssige Reibung erfassen.

Viskose Dämpfung entspricht einem linearen, geschwindigkeitsproportionalen Dämpfungsgesetz. Wenn der Schwinger zudem ein lineares federgesetz aufweist, kännen samit wiederum die Differentialgleichungen in der Art der Gleichungen (2) und (3) angesetzt werden. $c$ wird dabei als konstant vorausgesetzt und Dämpfungskoeffizient genannt. Bei der dynamischen Berechnung von Baukonstruktionen wird meistens vom Ansatz der viskosen Dämpfung ausgegangen, da die Lösung der Schwingungsdifferentialgleichungen damit besonders vereinfacht wird.

Eine grosse Bedeutung hat der Begriff der kritischen Dämpfung $c_{k r}$. Für einen Einmassenschwinger gilt bei freier Schwingung:

$$
c_{k r}=2 \cdot \sqrt{k \cdot m}
$$


Für $c \geqslant c_{k r}=2 \cdot \sqrt{k \cdot m}$ entsteht keine Schwingung, der Körper strebt ohne periodische Bewegung direkt die Ruhelage an. Für $c<c_{k r}=2 \cdot \sqrt{k \cdot m}$ (üblicher Fall) führt der körper eine periodische Bewegung aus, die je nach der Grösse der Dämpfung mehr oder weniger rasch der Ruhelage zustrebt.

Sehr häufig wird als Mass für die viskose Dämpfung das sogenannte Dämpfungsmass $\xi$, d.h. das Verhältnis der vorhandenen Dämpfung c zur kritischen Dämpfung $c_{k r}$ eingesetzt:

$$
\xi=\frac{c}{c_{k r}}
$$

Nebst dem Dämpfungskoeffizienten $c$ und dem Dämpfungsmass $\xi$ werden oft auch das logarithmische Dekrement $v$, der Verlustfaktor d und die Dämpfungskapazität $\Psi$ verwendet. Der Zusammenhang dieser Kenngrössen mit dem oben eingeführten Dämpfungskoeffizienten resp. dem Dämpfungsmass ist aus Bild 14 ersichtlich.

\section{b) Hysteresis-Dämpfung (Plastifizierungseffekte)}

Durch das Modell der Hysteresisdämpfung lassen sich vor allem die Plastifizierungseffekte erfassen.

In Bild 3 ist die elastische Rückstellkraft $R$ eines Einmassenschwingers in Funktion der Verschiebung $z$ aufgetragen. Bei einem elastischen, ungedämpften Schwinger erfolgt die Bewegung entlang der Geraden CA. Die schraffierte Fläche F zwischen der z-Achse und der Geraden $O A$ entspricht der maximalen potentiellen Energie, die im System gespeichert ist. Ohne Dämpfung erfolgt während jeder Periode ein vollständiger Austausch von kinetischer und potentieller Energie. Im Punkt $O$ ist die kinetische Energie $=F$, im Punkt $A$ ist die potentielle Energie $=\mathrm{F}$.

Ist zusätzlich eine Hysteresisdämpfung vorhanden, so verläuft die Bewegung nicht mehr entlang der Geraden CA, sondern es bildet sich eine Hysteresiskurve ABCD (gestrichelt). Es ist somit eine nichtlineare Beziehung zwischen Rückstellkraft und Verschiebung vorhanden. Je grösser die Dämpfung, desto grösser wird die von der Hysteresiskurve umschlossene Fläche. Bei jedem Schwingungszyklus wird dem System die der umschlossenen Fläche entsprechende Energie entzogen. Sofern diese Energie nicht fortwährend ersetzt, d.h. neu zugeführt wird, nimmt die Auslenkung mit jedem Zyklus ab (z.B. Ausschwingversuch).

Dieser Schwinger mit einer nichtlinearen Beziehung zwischen Rückstellkraft und Verschiebung kann bei der rechnerischen Behandlung im allgemeinen ersetzt werden durch einen linearen Schwinger mit viskoser Dämpfung, dessen Federgesetz entlang der Geraden CA verläuft. Die Energiedissipation des.nichtlinearen Schwingers wird ersetzt durch eine äquivalente viskose Dämpfung des linearen Schwingers, so dass sowohl der nichtlineare als auch der lineare Schwinger dieselben Perioden haben und bei beiden während eines Schwingungszyklus dieselbe Energie dissipiert wird.

Dịe Berechnung des äquivalenten viskosen Dämpfungsmasses $\xi$ kann entsprechend der Beziehung

$$
\xi=\frac{1}{2 \pi} \cdot \frac{\text { Fläche ABCDA }}{\text { Fläche DAE + Fläche OCF }}
$$

vorgenommen werden.

c) Coulomb-Dämpfung (Trockene Reibung)

Mit dem Modell der Coulomb-Dämpfung kann die Trockene Reibung erfasst werden. 
In Bild 4 ist das Modell eines Einmassenschwingers mit Coulomb-Dämpfung dargestellt. Die bewegungshemmende Reibungs- resp. Dämpfungskraft $F_{\mathrm{C}}$ hängt von der Normalkraft $N$ zwischen dem bewegten Körper und der Oberfläche, auf der er sich verschiebt sowie vom Gleitreibungskoeffizienten $\mu$ ab. Damit wird die Reibungs- resp. Dämpfungskraft $F_{C} z u$

$$
F_{c}=\mu \cdot N(z, \dot{z}, \ddot{z}) \cdot(-\operatorname{sgn} \dot{z})
$$

Der Term -sgn $\dot{z}$ gibt an, dass die Reibungskraft $F_{c}$ stets der Richtung der Relativgeschwindigkeit $\dot{z}$ entgegengesetzt wirkt.

Die resultierende Schwingungsdifferentialgleichung ist nichtlinear.

Für einen Einmassenschwinger mit linearem Federgesetz und Coulomb-Dämpfung ergibt sich somit die folgende Bewegungsgleichung

$$
m \cdot \ddot{z}+\mu \cdot N \cdot \operatorname{sgn} \dot{z}+k \cdot z=P(t)
$$

\subsubsection{Möglichkeiten zur experimentellen Bestimmung der Dämpfung}

\section{a) Ausschwingversuche}

In Bild 5 ist der theoretische Verlauf der Verschiebung $z$ während eines Ausschwingversuches in Funktion der Zeit angegeben.

Beim Ausschwingversuch wird dem Prüfkörper, z.B. einem Balken, eine Anfangsdurchbiegung $z_{0}$ aufgezwungen. Wird die Verbindung, mit welcher diese Verschiebung aufgebracht wurde, plötzlich gelöst, so kann der Prüfkörper eine freie Schwingung ausführen. Anstelle einer Anfangsverschiebung kann dem Prüfkörper auch ein Stoss erteilt werden, worauf er nach dem Abklingen der durch den Stoss angeregten Oberschwingungen ebenfalls frei ausschwingt. Die freie Schwingung kann nur in einer. Grundfrequenz und Grundform erfolgen, die durch die anfänglich aufgezwungene Verschiebung bzw." den Ort des Stosses bestimmt werden. Je nach der Grösse der Dämpfung klingt, die freie Schwingung mehr oder weniger stark ab.

Ausgehend vom Modeli der viskosen Dämpfung wird beim. Ausschwingversuch üblicherweise als Mass für die Dämpfung das logarithmische Dekrement v verwendet, das aus dem Verhältnis aufeinander folgender maximaler Amplituden mit der Beziehung

$$
\vartheta=\frac{1}{n} \cdot \ln \frac{z_{m}}{z_{m+n}}
$$

berechnet wird. Das logarithmische Dekrement v kann mit

$$
\xi=\frac{c}{c_{k r}}=\frac{\vartheta}{2 \pi}
$$

auf einfache Art in das oben eingeführte Dämpfungsmass $\xi$ umgerechnet werden.

Unter der Annahme einer viskosen Dämpfung lautet die Differentialgleichung eines Einmassenschwingers bei einer freien Schwingung $P(t)=0$

$$
m \cdot \ddot{z}+c \cdot \dot{z}+k \cdot z=0
$$

Mit der Kreisfrequenz $w$ des ungedämpften Schwingers

$$
\omega=\sqrt{\frac{k}{m}}
$$

erhält man 
$m \cdot \ddot{z}+2 \cdot m \cdot \xi \cdot w \cdot \dot{z}+k \cdot z=0$

Mit den Anfangsbedingungen

$$
\begin{aligned}
& z(t=0)=z_{0} \\
& \dot{z}(t=0)=0
\end{aligned}
$$

und der Kreisfrequenz $\omega^{\prime}$ des gedämpften Schwingers

$$
\omega^{\prime}=\omega \cdot \sqrt{1-\xi^{2}}
$$

ergibt sich als Lösung von Gl. (13)

$$
z(t)=z_{0} \cdot e^{-\xi \omega t} \cdot\left(\cos \left(\omega^{\prime} t\right)+\frac{\omega}{\omega^{\prime}} \cdot \sin \left(\omega^{\prime} t\right)\right)
$$

Zur Kontrolle des Modells des viskosen Dämpfung kann wie folgt vorgegangen werden (vgl. Bild 6): Auf der Abszisse des abgebildeten Diagramms werden die Anzahl der Schwingungsperioden $n$ aufgetragen, auf der Ordinate der natürliche Logarithmus der beim Ausschwingversuch nach $n$ Perioden erzielten maximalen Amplitude. Ist die Verbindungslinie durch die einzelnen Punkte eine Gerade, so ist die Annahme der viskosen Dämpfung korrekt, andernfalls ergibt sich eine gekrümmte Linie. Die Neigung der Geraden entspricht der Grösse des logarithmischen Dekrementes $v$.

\section{b) Resonanzversuche}

In Bild 7 ist der theoretische Verlauf einer Resonanzkurve eines Zweimassenschwingers abgebildet.

Eine Resonanzkurve entsteht, wenn ein Prüfkörper einer erzwungenen Schwingung $(P(t) \neq 0)$ unterworfen wird, bei welcher die Erregerfrequenz $\Omega$ kontinuierlich variiert wird. Werden die resultierenden maximalen Verschiebungen $z$ oder Dehnungen $\varepsilon$ im Frequenz-AmplitudenDiagramm aufgetragen, so entstehen dort Amplitudenmaxima, wo die Erregerfrequenz gleich einer Eigenfrequenz des Prüfkörpers ist (Resonanz). Je nach dem Verlauf des Federgesetzes und der Grässe der Dämpfung ändert sich die Form der Resonanzkurve. Je kleiner die Dämpfung ist, desto grösser werden die Amplituden und desto schmaler wird die Spitze im Resonanzbereich.

In Bild $\theta$ ist der theoretische Verlauf der Resonanzkurve für einen Einmassenschwinger mit mit unterlinearem, linearem und überlinearem Federgesetz dargestellt.

Bei linearem Verhalten (lineares Federgesetz, viskose Dämpfung) ist die Resonanzkurve vertikal und weitgehend symmetrisch zur vertikalen Achse durch das Amplitudenmaximum bei Resonanz.

Bei nichtlinearem Verhalten (nichtlineares Federgesetz) wird die Eigenfrequenz amplitudenabhängig.

Bei unterlinearem Verhalten (unterlineares Federgesetz), d.h. wenn die Durchbiegung stärkèr zunimmt als die Last, wird der Versuchskörper mit zunehmender Durchbiegung "weicher", womit die Eigenfrequenz sinkt. Deshalb kippt die Resonanzkurve nach links.

Bei ueberlinearem Verhalten (überlineares Federgesetz), wo die Durchbiegung langsamer zunimmt als die Last, verhält es sich umgekehrt. Mit zunehmender Durchbiegung wird der Versuchskörper "steifer", womit die Eigenfrequenz zunimmt und die Resonanzkurve nach rechts kippt. 
Bei kleiner Dämpfung und stark nichtlinearem Verhalten wird sehr rasch ein Zustand erreicht, bei welchem einer Erregerfrequenz zwei oder sogar drei mägliche Amplituden entsprechen. Bei der experimentellen Bestimmung der Resonanzkurve kann der Schwinger somit plötzlich von einem Niveau mit kleinen Amplituden auf ein Niveau mit grossen Amplituden (oder umgekehrt) springen.

Die Differenzialgleichung für einen linearen Einmassenschwinger mit viskoser, geschwindigkeitsproportionaler Dämpfung, der einer sinusförmig verlaufenden Erregerkraft unterworfen wird, hat die form

$$
m \cdot \ddot{z}+2 \cdot m \cdot \xi \cdot \omega \cdot \dot{z}+k \cdot z=P_{0} \cdot \sin (\Omega t)
$$

'Mit

$$
\omega=\sqrt{\frac{k}{m}} \quad \text { und } \quad \omega^{\prime}=\omega \cdot \sqrt{1-\xi^{2}}
$$

und dem Frequenzverhältnis $\beta$

$$
\beta=\frac{\Omega}{\omega}
$$

erhält man als Lösung von Gl. (15)

$$
\begin{aligned}
z(t) & =e^{-\xi \omega t} \cdot\left(A \cdot \sin \left(\omega^{\prime} t\right)+B \cdot \cos \left(\omega^{\prime} t\right)\right)+ \\
& +\frac{P}{k} \frac{1}{\left(1-\beta^{2}\right)^{2}+(2 \cdot \xi \cdot \beta)^{2}} \cdot\left[\left(1-\beta^{2}\right) \cdot \sin (\Omega t)-2 \cdot \xi \cdot \beta \cdot \cos (\Omega t)\right]
\end{aligned}
$$

Der erste Teil von Gl. (16) wird durch den Term e $e^{-\xi \omega t}$ mit der Zeit "herausgedämpft". Der zweite Teil stellt die stationäre Schwingung $z(t)$ stationär dar, die durch die Erregerkraft aufrecht erhalten wird. Die maximale stationäre Amplitude $z_{\text {max }}$ beträgt

$$
z_{\max }=\frac{P_{O}}{k} \cdot\left[\left(1-\beta^{2}\right)^{2}+(2 \cdot \xi \cdot \beta)^{2}\right]^{-V / 2}
$$

Mit dem Phasenwinkel $\theta$

$$
\theta=\tan ^{-1} \frac{2 \cdot \xi \cdot \beta}{1-\beta^{2}}
$$

welcher ein Mass ist für den Zeitabstand, mit dem die "Antwort" des Schwingers $z(t)$ der Erregerkraft $P(t)$ nacheilt, kann der stationäre Teil $z(t)$ stationär von Gl. (16) vereinfacht werden als

$$
z(t)_{\text {stationär }}=z_{\max } \cdot \sin (\Omega t-\theta)
$$

Der Vergrösserungsfaktor $V_{\max }$ gibt an, wievielmal grösser die dynamische Amplitude $z_{\max }$ gegenüber der unter gleich grosser Kraft erzielten statischen Verschiebung $z_{\text {statisch }}$ ist.

$$
v_{\text {max }}=\frac{z_{\text {max }}}{z_{\text {statisch }}}=\frac{z_{\max }}{P_{0} / k}=\left[\left(1-\beta^{2}\right)^{2}+(2 \cdot \xi \cdot \beta)^{2}\right]^{-1 / 2}
$$

In Bild $g$ ist der Vergrösserungsfaktor $V$ für einen linearen Einmassenschwinger und für verschiedene Dämpfungsmasse $\xi$ in Funktion des Frequenzverhältnisses $\beta$ angegeben. In Bild 10 ist die Phasenverschiebung $\theta$ für denselben Schwinger und dieselben Dämpfungsmasse $\xi$ wie in Bild 9 und ebenfalls in Funktion von $\beta$ angegeben. Bei Resonanz $(\beta=1)$ beträgt die Phasenverschiebung unabhängig vom Dämpfungsmass $90^{\circ}$. 
Zur Bestimmung der Dämpfung aus der Resonanzkurve können verschiedene Verfahren zur Anwendung kommen:

- Berechnung aus dem Vergrösserungsfaktor V (Methode der Resonanzverstärkung):

Die Dämpfung lässt sich aus der maximalen Amplitude bei Resonanz berechnen. Das Verhältnis $\xi$ der vorhandenen zur kritischen Dämpfung beträgt

$$
\xi=\frac{1}{2 V_{\max }} \text { (Näherung); } \quad V_{\max }=\frac{1}{2 \cdot \xi \cdot \sqrt{1-\xi^{2}}} \text { (genau) }
$$

Dieses Vorgehen hat den Vorteil, dass für die Bestimmung der Dämpfung nur die Kenntnis der maximalen Amplitude notwendig ist, die Form der Resonanzkurve jedoch nicht bekannt sein muss. Dem steht der Nachteil gegenüber, dass die statische Verschiebung $z_{\text {statisch }}$ unter der last $P_{0}$ und damit die Steifigkeit $k$ oft nur ungenau bestimmt werden kann.

- Berrechnung aus der Form resp. Breite der Resonanzkurve (Methode der halben Bandbreite): Bild 11 soll die im Versuch gemessene Resonanzkurve eines linearen Einmassenschwingers darstellen. Aus der gemessenen maximalen Amplitude $z_{\max }$ bei Resonanz und dem bekannten Verlauf der Resonanzkurve können die zu den beiden reduzierten Amplituden $z_{\text {max }} / \sqrt{2}$ gehörigen Frequenzen $f_{1}$ und $f_{2}$ resp. $\beta_{1}$ und $\beta_{2}$ herausgelesen werden. Aus diesen Grössen kann das Dämpfungsmass zu

$$
\xi=\frac{1}{2} \cdot\left(\beta_{2}-\beta_{1}\right) \quad \text { oder } \quad \xi=\frac{f_{2}-f_{1}}{f_{2}+f_{1}}
$$

berechnet werden.

Der bei der zuerst genannten Methode erwähnte Nachteil fällt weg, dafür muss die Form der Resonanzkurve experimentell genau ermittelt werden, was häufig mit Schwierigkeiten verbunden ist.

- Berechnung aus dem Energieverlust pro Periode:

Damit der Energieverlust pro Periode infolge der Dämpfung bestimmt werden kann, muss die Phasenverschiebung $\theta$ zwischen Störkraft $P(t)$ und resultierender Verschiebung $z(t)$ genau gemessen werden können. Im Resonanzfall beträgt die Phasenverschiebung genau $90^{\circ}$. Daraus ergibt sich folgendes Vorgehen:

Die Erregerfrequenz wird variiert, bis die Phasenverschiebung $\theta=90^{\circ}$ beträgt. Im stationären Zustand ist die durch die Erregerkraft zugeführte Energie gleich der durch das Schwingsystem dissipierten Energie (vgl. Bild 12). Die Dämpfungskraft $F_{D}$ wird somit durch die Erregerkraft $P(t)$ gerade kompensiert. Daraus ergibt sich der Dämpfungskoeffizient $c$ aus dem Verhältnis der maximalen Dämpfungskraft $F_{D \text {, max }}$ zur maximalen Geschwindigkeit $\dot{z}_{\max } z u$

$$
c=\frac{F_{D, \text { max }}}{\dot{z}_{\max }}=\frac{P_{\text {max }}}{w \cdot z_{\max }}
$$

Eine andere Möglichkeit ergibt sich, wenn im Resonanzfall $\left(\theta=90^{\circ}\right)$ das Kraft-Verschiebungs-Diagramm aufgetragen wird (Bild 13). Wenn das Dämpfungsgesetz dem linear-viskosen Ansatz folgt, resultiert als Figur eine Ellipse. Bei anderen Dämpfungsverhalten ergibt sich eine beliebige geschlossene Kurve (gestrichelt). In diesem Fall kann in die geschlossene Kurve eine flächengleiche Ellipse eingezeichnet werden, welche auf der Abszisse ebenfalls durch den Punkt $z_{\max }$ geht. Mit Hilfe der sich innerhalb der Figur ergebenden Fläche $E$

$$
E=P_{\max } \cdot z_{\max } \cdot \pi
$$


kann der Dämpfungskoeffizient c berechnet werden zu

$$
c=\frac{E}{\pi \cdot w \cdot z_{\max }^{2}}
$$

Wenn das Dämpfungsverhalten nicht-linear-viskos ist, wird mit der GI. (24) für c ein analoger viskoser Dämpfungskoeffizient gefunden. Für E muss dabei die Fläche innerhalb der gestrichelten Kurve eingesetzt werden.

Der ermittelte Dämpfungskoeffizient ist frequenzabhängig.

\subsubsection{Umrechnung der Dämpfungskenngrössen}

Aus Bild 14 können die Umrechnungsfaktoren der für dynamische Berechnungen am häufigsten verwendeten Dämpfungskenngrössen entnommen werden. Nebst den hier angegebenen Kenngrössen für die Dämpfung können noch weitere Grössen verwendet werden, z.日. Verlustwinkel, Halbwertbreite, relative Dämpfung, Abklingungskonstante, Dämpfungskonstante, etc. Für deren Umrechnungen in andere Grössen sei auf [5] verwiesen.

2.3 Frühere Versuche zur Bestimmung der Dämpfungseigenschaften von Stahlbeton- und Spannbeton-Bauteilen

Nebst zahlreichen Berichten über dynamische Versuche, in welchen an Stahl- und Spannbetonkonstruktionen (Brücken, Decken, Türme, etc.) Dämpfungswerte gemessen wurden, standen diverse weitere Angaben über früher durchgeführte Versuche an Stahl- und Spannbetonbalken zur Verfügung. Versuche an schlaff armierten Stahlbetonbalken wurden z.B. von Bock [8], Ehlers [9], Lenk [10] und Penzien [11] durchgeführt. Denkhaus und Dück [12], Müller [13], Fritz-de-la-Orta [14] und Penzien [11] nahmen Versuche an Spannbetonbalken vor.

Die meisten der in den oben erwähnten Versuchen geprüften Versuchsbalken hatten relativ geringe Abmessungen und erste Eigenfrequenzen im Bereich zwischen 35 und $120 \mathrm{~Hz}$. Die Balken wurden teils in Ausschwingversuchen, teils in Resonanzversuchen geprüft. Die dabei erzeugten Verschiebungen waren jeweils sehr gering. Die gemessenen Werte für die Dämpfung streuen innerhalb eines sehr weiten Bereichs. Aus den zitierten Versuchen lässt sich im wesentlichen Folgendes herauslesen:

- Die Dämpfung von Stahlbetonbalken wird stark von der Rissbildung beeinflusst. Gerissene Balken weisen gegenüber ihrem ungerissenen Zustand in der Regel eine grössere Dämpfung auf. Gerissene Balken mit hoher Betongüte sowie mit starker und glatter Bewehrung ergeben besonders niedrige Werte für die Dämpfung.

- Die Dämpfung von Spannbetonbalken im ungerissenen Zustand ist meistens geringer als bei schlaff armierten und gerissenen Betonbalken. Mit zunehmender zentrischer Spannung aus der Vorspannung nimmt die Dämpfung im allgemeinen leicht ab. Erst bei sehr hohen Spannungen infolge von Vorspannung wird wieder ein Ansteigen der Dämpfung beobachtet.

- Versuche über die Dämpfung von Stahlleichtbetonbalken und von Spannleichtbetonbalken sowie von teilweise vorgespannten Beton- und Leichtbetonbalken sind nicht bekannt geworden. 
3. VERSUCHSBALKEN

In einem ersten Abschnitt werden die hauptsächlichsten Ueberlegungen und Zusammenhänge aufgezeigt, die zur gewählten Konzeption der Versuche führten. In einem zweiten Teil werden die Versuchskörper und die Eigenschaften der verwendeten Materialien im einzelnen beschrieben.

\subsection{Versuchsplanung}

Aus der in Abschnitt 2.2.2 angegebenen Darstellung der Dämpfungsursachen und den in $A b-$ schnitt 2.3 dargelegten Resultaten aus früher durchgeführten Versuchen ist ersichtlich, dass die dynamischen Eigenschaften von Stahlbetonkonstruktionen durch eine Vielzahl von nur wenig erforschten Parametern beeinflusst werden. Grosse Bedeutung scheinen der Rissbildung und der Höhe der Beanspruchung sowie dem Vorspanngrad zuzukommen.

Wegen der grossen Anzahl von Einfluss-Parametern und deren zum Teil gegenseitigen Abhängigkeit wurde angestrebt, anhand möglichst einfacher Versuche den jeweiligen Einfluss der wichtigsten einzelnen Parameter auf die dynamischen Eigenschaften von Leichtbeton- und Betonbalken abzuklären. In der Versuchsserie, über die hier berichtet wird, wurde der Einfluss der Vorspannung ausgeklammert. Vorgespante Balken sollen in einer späteren Serie geprüft werden.

\subsubsection{Statisches System und wichtigste Abmessungen}

Mit den hier beschriebenen Versuchen sollte insbesondere das dynamische Verhalten unter Biegebeanspruchung untersucht werden. Die Prüfkörper wurden als einfache Balken statisch bestimmt gelagert. Damit konnte auch gewährleistet werden, dass vorgängig und nachträglich vorgenommene theoretische Untersuchungen mit vertretbarem Aufwand und genügender Genauigkeit durchgeführt werden konnten.

Die Eigenfrequenzen der Prüfkörper sollten etwa den an ausgeführten Tragwerken festgestellten Eigenfrequenzen entsprechen, damit aus der vorgenommenen Untersuchung praxisnahe Folgerungen gezogen werden können. Es wurden deshalb möglichst tiefe erste Eigenfrequenzen im Bereich von 2 bis $10 \mathrm{~Hz}$ angestrebt.

Die Höhe der Eigenfrequenzen wird nicht nur durch die Schlankheit und das statische System des objektes, sondern ebenso durch dessen absolute Grösse und das gewählte Material bestimmt. Dies geht aus folgendem Zusammenhang hervor: Die Eigenfrequenzen $\omega_{i}$ eines einfachen Balkens mit konstanter, verteilter Masse q je Längeneinheit und konstanter Biegesteifigkeit EI sind gegeben durch

$$
\omega_{i}=\frac{i^{2} \cdot \pi^{2}}{l^{2}} \cdot \sqrt{E I \cdot \frac{g}{q}} \quad i=1,2, \ldots, n
$$

wobei 1 die Stützweite des Trägers und g die Erdbeschleunigung bedeuten.

Für einen Rechteckquerschnitt mit der Höhe $H$ und der Breite B wird mit

$$
\begin{aligned}
& I=c_{1} \cdot B \cdot H^{3}, \quad q=c_{2} \cdot B \cdot H \quad \text { und } c=f\left(c_{1}, c_{2}\right) \\
& \omega=c \cdot \frac{1}{1^{2}} \sqrt{\frac{H^{3}}{H}}=c \cdot \frac{H}{1} \cdot \frac{1}{1}
\end{aligned}
$$


In G1. (26) stellt der Term H/I den Einfluss der Schlankheit und der zweite Term $1 / 1$ den Einfluss der absoluten Grösse des Trägers dar.

In Bild 15 sind die nach G1. (25) theoretisch berechneten ersten Eigenfrequenzen eines Betonbalkens in Funktion der Stützweite 1 für zwei verschiedene Schlankheiten $H / l$ aufgetragen.

Aus dem Diagramm ist ersichtlich, dass das Verhältnis $H / 1$ relativ gering, die Länge der Versuchsbalken jedoch recht gross gewählt werden musste, um innerhalb des gewünschten Frequenzbereiches zu liegen.

Um einen breiteren Frequenzbereich abdecken zu können, wurden alle Prüfkörper auch mit . verkleinerter Stützweite und entsprechenden Kragarmen geprüft (vgl. Bild 16). Dank der so erhöhten Systemsteifigkeit konnten die Eigenfrequenzen praktisch auf das Doppelte der am einfachen Balken ohne Kragarme erzielbaren Eigenfrequenzen gesteigert werden. Die Trägerhöhe musste so gewählt werden, dass die erforderliche Schlankheit eingehalten wurde und die Balken in der anfänglichen Ruhelage noch weitgehend ungerissen waren. Zudem mussten in der Konstruktionspraxis übliche Grössenordnungen - auch bezüglich Durchmesser der Armierungsstäbe - erreicht werden können.

Die Breite der Balken musste relativ klein sein, um die Masse der Versuchskörper und damit auch die notwendige Erregerkraft gering halten zu können.

Aufgrund der angeführten Ueberlegungen wurden als Versuchskörper einfache Balken der Gesamtlänge $L=8.40 \mathrm{~m}$, der Breite $B=0.40 \mathrm{~m}$ und der Hähe $H=0.24 \mathrm{~m}$ gewählt, die mit den Stützweiten $1=7.88 \mathrm{~m}$ und $1=4.84 \mathrm{~m}$ geprüft wurden.

\section{1 .2 Armierungen}

Die Balken dieser Versuchsserie wurden ausschliesslich schlaff armiert. Die Grösse der Druckarmierung wurde gleich derjenigen der Zugarmierung gewählt, damit negative Momente infolge negativer (nach oben gerichteter) Durchbiegungen oder infolge auskragender Balkenenden aufgenommen werden konnten. Die Armierungsgehalte $\mu$ wurden so gewählt, dass in der Praxis übliche Werte vorhanden waren und keine spröden Brüche auftreten konnten

$\left(\mu_{\text {krit }}<\mu<\mu_{\text {Grenz }} d . h\right.$. Betonbruch während Stahlfliessen). Die Bügelarmierung wurde überall so ausgebildet, dass ein Schubbruch von vorneherein ausgeschlossen werden konnte.

\section{1 .3 Betonarten}

Der Zielsetzung gemäss sollte nebst normalem Kiesbeton ein Leichtbeton verwendet werden, der in der Praxis für tragende Konstruktionen zum Einsatz kommt. Zusätzlich sollte ein Leichtbeton mit noch geringerem Raumgewicht zur Anwendung kommen, der für weniger stark beanspruchte Elemente verwendet wird.

\section{1 .4 Zusatzmassen}

Während des Prüfvorganges wurden zusätzliche Massen $\Delta M_{1}=152.4 \mathrm{~kg} / \mathrm{m}$ und $\Delta M_{2}=295.2 \mathrm{~kg} / \mathrm{m}$ auf die Prüfkörper aufgebracht (vgl. Bild 17). Damit konnten zwei Effekte erzielt werden: Einerseits wurde durch die Zusatzmassen die Eigenfrequenz der Versuchsbalken verringert. Damit konnte die angestrebte grosse Breite des untersuchten Frequenzbereichs nach unten erweitert werden. Andererseits hatte das Aufbringen der Zusatzmassen zur Folge, dass die Ruhelage der Versuchsbalken und damit die Beanspruchungen und Rissweiten verändert wurden. Da zu erwarten war, dass das Dämpfungsverhalten sehr stark durch die auftretenden Risse beeinflusst würde, konnte so der Einfluss der veränderten Ruhelage resp. Rissöffnung auf die Dämpfung erfasst werden. 


\subsubsection{Prüfarten}

Von den Möglichkeiten zur Ermittlung von Eigenfrequenz und Dämpfung wurden Ausschwingversuche und Resonanzversuche (vgl. Abschnitt 2.2.4) als am zweckmässigsten erachtet. Zusätzlich zu den dynamischen Versuchen wurden sämtliche Balken auch statisch belastet, um deren Steifigkeit genauer zu ermitteln.

Die verwendeten Belastungseinrichtungen werden im Abschnitt 4.1 beschrieben.

\subsection{Beschreibung der Versuchsbalken}

\subsubsection{Allgemeines}

Tabelle 1 gibt eine Uebersicht über die Versuchsserie. Abmessungen und Armierungen gehen aus Bild 18 hervor. Die Gesamtlänge von $L=8.40 \mathrm{~m}$, die Breite $B=0.40 \mathrm{~m}$ und die Höhe von $H=0.24 \mathrm{~m}$ waren bei allen Balken gleich.

Der Längsarmierungsgehalt $\mu$ der Balken B1, B2 und B3 betrug $0.57 \%$, $1.01 \%$ und $1.57 \%$, was je 4 Stäben Ø 12, Ø 16 und Ø $20 \mathrm{~mm}$ entsprach. Die Balken LB1, LB2 und LB3 entsprachen genau den Balken $81, B 2$ und 83 , waren jedoch aus einem Leichtbeton mit hochfesten Lecahade-Leichtzuschlagstoffen und einem Raumgewicht von ca. $1.76 \mathrm{t} / \mathrm{m}^{3}$ (ohne Armierung) hergestellt. Der Balken LB4 wies die gleiche Längsarmierung auf wie die Balken B2 und LB2. Hier kam jedoch ein Leichtbeton mit nọrmalen Leca-Leichtzuschlagstoffen und einem Raumgewicht von $1.53 \mathrm{t} / \mathrm{m}^{3}$ (ohne Armierung) zur Anwendung. Alle Balken wiesen dieselbe Bügelarmierung auf. Die Mindestüberdeckungen der Armierung betrug $2 \mathrm{~cm}$.

Für die Herstellung der Balken wurde eine Holzschalung verwendet. Die pro Balken notwendige Betonmenge wurde in einem Zwangsmischer in drei Mischchargen hergestellt. Der Beton wurde nach dem Einbringen mit Nadelvitbratoren verdichtet. Jeweils eine Woche nach dem Betonieren wurden die Balken ausgeschalt und bis zum Einbau in die Versuchsanlage in der Betonierhalle gelagert.

\subsubsection{Armi erungsstahl}

Die Längs - und Bügelarmierungen bestanden aus kaltverformtem, hochwertigem Armierungsstahl (TOR). In einem Zugversuch wurden von jedem Durchmesser an je 3 Stäben die Span-

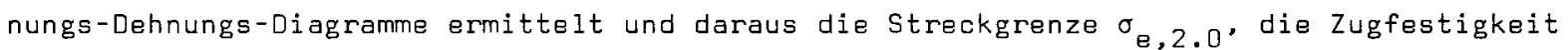
$B_{z}$ und der E-Modul $E_{e}$ herausgelesen. Am geprüften Armierungsstahl wurden die Bruchdehnungen $\lambda$ und die Brucheinschnürung $\Psi$ bestimmt. Die gemittelten Werte sind in Tabelle 2 angegeben.

Die Spannungs-Dehnungs-Diagramme wurden mittels einer dehnungsgesteuerten Zugmaschine aufgenommen. Naşh einer Vorbelastung bis ca. $0.5 \sigma_{8,2.0}$ wurden die Armierungsstäbe wieder bis ca. $0.2 \sigma_{e, 2.0}$ entlastet und aus den gemessenen Spannungs- resp. Dehnungsänderungen der E-Modul bestimmt. Daran anschliessend wurden die $\sigma-\varepsilon-D i a g r a m m e ~ a u f g e z e i c h n e t, ~ w o b e i$ die Dehngeschwindigkeit jeweils auf $0.5 \%$ /Minute festgelegt war. Die so erhaltenen, gemittelten $\sigma-\varepsilon$-Diagramme sind in Bild 19 wiedergegeben.

Für den verwendeten Armierungsstahl standen keine wählerkurven zur Verfügung, aus denen der Festigkeitsabfall in Funktion der Anzahl Lastwechsel hätte bestimmt werden können. Es ist allerdings fraglich, ob aus solchen Kurven Schlüsse für das Ermüdungsverhalten auch von einbetonierten Armierungsstählen gezogen werden dürfen. Zwar war dieses Verhalten nicht Gegenstand der vorliegenden Untersuchung. Da jedoch die Ober- und Unterspannung während der Versuche zum Teil sehr weit auseinander lagen und auch das Total der Anzahl Lastwechsel recht gross war, konnte ein Ermüdungsbruch nicht von vorneherein ausgeschlossen werden. Untersuchungen zur Ermüdungsfestigkeit von Stahlbetonbalken wurden 
zum Beispiel von Soretz [15], [16] durchgeführt. Wegen der während des ganzen Versuchs variierenden Beanspruchungshöhe (Ober- und Unterspannung) sind diese Resultate nicht direkt anwendbar. Man beschränkte sich deshalb darauf, bei jeder Beanspruchungsstufe die dazu gehörige Anzahl Lastwechsel zu registrieren.

In den Bildern 20a, 20b und 20c sind die pro Beanspruchungsstufe (vgl. Abschnitt 4.2) erreichten Spannungen $\sigma_{e}$ in der Längsarmierung dargestellt. Der eingezeichnete Punkt bezeichnet die jeweilige Spannung in der Ruhelage des Balkens $\sigma_{e, 0}$ und es sind die bei Resonanz erzielten maximalen und minimalen Spannungen und damit die Schwingweite eingetragen. Am oberen Rand jeder Darstellung ist die bei der zugehörigen Beanspruchungsstufe erreichte Anzahl Lastwechsel aufgeführt. Dabei gilt es jedoch zu beachten, dass sich die angegebene Lastwechselzahl auf die gesamte Beanspruchungsstufe bezieht (zwei Resonanzkurven mit steigender und fallender Frequenz, vgl. Abschnitt 4.2), die eingezeichnete maximale Schwingweite jedoch nur bei einem Bruchteil der hier angegebenen Lastwechsel erreicht wurde.

Ein Ermüdungsbruch der Stahleinlagen ist trotz der hohen Schwingweiten und beträchtlicher Lastwechselzahlen nie aufgetreten.

\subsubsection{Leichtbeton und Beton}

Leichtbetone: In Tabelle 3 ist die Zusammensetzung der für die Balken LB1, LB2, LB3 und LB4 verwendeten Leichtbetone angegeben. Die Mischrezepte entsprachen den Angaben der Firma Hunziker \& Cie AG, Zürich. Als Leichtzuschlagstoffe wurden Leca-hade- resp. LecaLeichtzuschlagstoffe (Blähton) verwendet. Als Bindemittel wurden normaler Portlandzement sowie hydraulischer Kalk zur Verbesserung der Verarbeitbarkeit beigegeben.

Beton: In Tabelle 3 ist die Zusammensetzung des für die Balken B1, B2 und B3 verwendeten Frischbetons angegeben. Als Zuschlagstoffe wurden Sand und Kies, getrennt nach vier Komponenten, beigegeben. Vor dem Betonieren wurden die Siebkurve und der Wassergehalt der Zuschlagstoffe bestimmt. Die Siebkurve der Mis'chung entsprach ungefähr der Fuller-Kurve. Als Bindemittel wurde normaler Portlandzement verwendet.

Untersuchungen: Die erhaltenen Resultate sind in Tabelle 4 zusammengestellt. Zur Bestimmung der Festigkeiten wurden beim Betonieren jedes Bäkens aus drei verschiedenen Mischchargen Würfel $(15 \times 15 \times 15 \mathrm{~cm})$ und Zylinder $(15 \mathrm{~cm} \emptyset$ und $30 \mathrm{~cm}$ Höhe) hergestellt. Im Alter von durchschnittlich 35 Tagen, d.h. nach Durchführung der Balkenversuche, wurden die Betonproben auf folgende Weise geprüft:

- An jeweils 9 bis 15 Würfeln mit $15 \mathrm{~cm}$ Kantenlänge wurden das Raumgewicht $\gamma$ und die Würfeldruckfestigkeit $\beta_{W}$ ermittelt.

- An 6 Zylindern je Balken (ausser bei LB4) wurden nebst dem Raumgewicht für verschiedene Beanspruchüngshöhen die E-Moduli als Sekantenmoduli bestimmt. Für $E_{5-50}$ wurde die Spannung bis auf $50 \mathrm{~kg} / \mathrm{cm}^{2}$ gesteigert und denach wieder bis auf $5 \mathrm{~kg} / \mathrm{cm}^{2}$ verringert. Bei der anschliessenden Wiederbelastung bis $50 \mathrm{~kg} / \mathrm{cm}^{2}$ wurden der Spannungs- und Dehnungszuwachs gemessen und daraus der E-Modul berechnet. Für $E_{5-100}, E_{5-150}$ und $E_{5-200}$ wurde entsprechend vorgegangen. Anschliessend wurde die Last bis zum Bruch gesteigert und daraus die Prismendruckfestigkeit $\beta_{p}$ erhalten.

- An weiteren 6 Zylindern je Balken (LB4: 3) wurden die Spannungs-Dehnungs-Diagramme ermittelt. Die Belastung wurde so aufgebracht, dass die Stauchung von Laststufe zu Laststufe innert einer Minute um jeweils ca. $0.25 \cdot 10^{-3}$ gesteigert wurde. Nach jeder Lasterhöhung wurde die Stauchung während zweier Minuten konstant gehalten. Durch diese Prüfmethode erhält man ein dynamisches $(\dot{\varepsilon} \neq 0)$ und ein quasi-statisches $(\dot{\varepsilon}=0)$ Spannungs-Dehnungs-Diagramm. In Bild 21 ist für alle drei Betonarten je ein BeispieI wiedergegeben. 


\subsubsection{Rechnerische Werte}

Querschnittswerte, Rissmoment und Plastisches Moment der Versuchsbalken sind aus Tabelle 5 ersichtich.

Für die Berechnung der ideellen Querschnittswerte wurde sowohl für den Leichtbeton als auch für den Beton das Verhältnis der in den Vorversuchen ermittelten E-Moduli eingesetzt und zwar:

$$
\begin{array}{ll}
\text { Leichtbeton: } & n=\frac{E_{e}}{E_{L B, 5-100}}=\frac{2 \cdot 100}{175}=12 \quad \text { (LB1, LB2, LB3) } \\
n & =\frac{E_{e}}{E_{L B, 5-100}}=\frac{2 \cdot 100}{130}=16 \quad \text { (LB4) } \\
\text { Beton: } & n=\frac{E_{B}}{E_{B, 5-100}}=\frac{2 \cdot 100}{350}=6 \quad(B 1, B 2, B 3)
\end{array}
$$

Das Rissmoment wurde mit der Biegezugfestigkeit $\beta_{b z}$ gemäss der in der Norm SIA 162 sowie in der zugehörigen Leichtbeton-Richtlinie Nr. 33 angegebenen Formeln berechnet:

$$
\begin{array}{ll}
\text { Leichtbeton: } & \beta_{b z}=2.0 \cdot \sqrt{\beta_{w}} \\
\text { Beton: } & B_{b z}=2.5 \cdot \sqrt{\beta_{w}}
\end{array}
$$

Für die Berechnung des plastischen Momentes wurden die Festigkeitswerte entsprechend dem jeweiligen Mittel der aus den Baustoffuntersuchungen gewonnenen Werte eingesetzt.

Tabelle 6 zeigt für die massgebenden Balkenquerschnitte die maximalen Beanspruchungen in der Ruhelage. Für die Spannungsberechnungen am gerissenen Querschnitt nach dem $n$-Verfahren wurden für $n$ ebenfalls die in den Gleichungen (27) angegebenen Werte eingesetzt.

Die verwendeten Abkürzungen und Symbole sind in der. Legende zu Tabelle 6 und im Abschnitt "Bezeichnungen" erläutert. 
Im ersten Unterabschnitt wird die verwendete Versuchseinrichtung beschrieben. Anschliessend wird der gewählte Versuchsablauf dargestelit. Im dritten und vierten Unterabschnitt wird über die vorgenommenen Messungen und die Methoden der Auswertung berichtet.

Die Versuchsserie wurde auf dem Aufspannboden im Forschungsgebäude der ETH-Hönggerberg durchgeführt. Bevor diese Serie in Angriff genommen werden konnte, wurde an der Eidgenössischen Materialprüfungs- und Versuchsanstalt (EMPA) in Dübendorf ein Vorversuch durchgeführt, wobei die vorgängig entwickelten Versuchs- und Messeinrichtungen ausprobiert und einer eingehenden Prüfung unterzogen werden konnten.

\subsection{Versuchsanlage}

Bild 22 zeigt eine Uebersicht über die gesamte Anlage während des Vorversuches in der EMPA. Alle Balken der Versuchsserie wurden in derselben Anlage geprüft. Auf der einen Seite war ein stehendes, festes Lager angeordnet, das nur Auflagerdrehwinkel zuliess; auf der anderen Seite war ein hängendes Pendellager angebracht, das nebst Verdrehungen auch Längsverschiebungen ermöglichte.

\subsubsection{Ausschwingvorrichtung}

Durch eine an der unteren Befestigungsplatte des Schwingungserregers angebrachte Spannschraube konnte dem Prüfbalken die gewünschte Anfangsdurchbiegung aufgezwungen werden. Bild 23 zeigt die gewählte, sehr einfache Ausklinkvorrichtung. Die Zugkraft der Spannschraube wurde durch einen Hebelmechanismus und ein vertikales Metallplättchen an den Aufspannboden abgegeben. Mit einem Hammerschlag auf das Metallplättchen liess sich die Verbindung lösen, worauf der Prüfbalken frei ausschwingen konnte.

\subsubsection{Schwingungserreger}

Der für die Erzeugung der erzwungenen Schwingung benützte Schwingungserreger ist in Bild 24 abgebildet. Eine genauere Beschreibung wird im Anhang 1 gegeben.

In einem Rahmen wird mittels eines durch eine Servohydraulik gesteuerten Zug-DruckZylinders eine Schwingmasse hin und her bewegt. Der Schwingungserreger wurde auf dem Versuchsbalken fest montiert. Die auf den Balken ausgeübten dynamischen Kräfte entsprechen den durch die Hin- und Herbewegung der Schwingmasse $\bar{m}$ entstehenden Trägheitskräften $m \bullet a ̈$. a ist gleich dem Weg der Masse $\bar{m}$, d.h. gleich dem Weg des Kolbens im Zug-DruckZylinder.

Zur Erzeugung der Resonanzschwingung wurde ein sinusförmiger Verlauf der Erregerkraft angestrebt.

Die maximale Erregerkraft $P_{\text {Err,max }}$ konnte aus dem Kolbenweg des Schwingungserregers

$$
a=a_{0} \cdot \sin (\Omega t)
$$

der bewegten Masse $\bar{m}$ und der eingestellten Erregerfrequenz $\Omega$ aus der Beziehung

$$
P_{\text {Err,max }}=\bar{m} \cdot a_{0} \cdot \Omega^{2}
$$

berechnet werden. Beim Durchfahren der einzelnen Resonanzkurven wurde die maximale Erregerkraft $P_{\text {Err,max }}$ jeweils konstant gehalten, indem bei jeder Frequenzänderung $\Delta \Omega$ der 
Kolbenhub a um einen entsprechenden Betrag $\Delta a$ korrigiert wurde.

Ein zwischen der Kolbenstange und der bewegten Masse des Schwingungserregers eingebautes, ringförmiges Kraftmesselement (vgl. Bild 24) diente der direkten Messung der Erregerkraft. Da jedoch für die Grösse der Erregerkraft nur die relative Bewegung der Schwingmasse zum Balken massgebend ist, das Kraftmesselement jedoch die Kraft infolge der totalen Beschleunigung der Schwingmasse misst, d.h. inklusive derjenigen des Balkens, lieferte die direkte Messung nur brauchbare Resultate, wenn der Schwingungserreger auf einer ruhenden Unterlage befestigt war. Nach Durchführung der Versuche wurde der Schwingungserreger auf dem Aufspannboden befestigt und die gemäss Gl. (29) berechnete Erregerkraft mit der anhand des Kraftmesselementes festgestellten Kraft verglichen. Die Uebereinstimmung war befriedigend.

\subsubsection{Statische Belastungsvorrichtung}

Zur genaueren Ermittlung der Balkensteifigkeit wurde nebst der dynamischen auch eine statische Belastungsvorrichtung angeordnet. Letztere ist in Bild 25 schematisch dargestellt. Die Versuchsbalken wurden an zwei Punkten im Abstand von je $1.0 \mathrm{~m}$ von der Balkenmitte belastet. Dazu wurden ein hydraulischer Zug-Druck-Zylinder, ein Lastverteilbalken sowie je zwei Zug-Druck-Stangen verwendet. Der Angriffspunkt der beiden Einzellasten wurde so gewählt, dass in Balkenmitte trotz unterschiedlichem Verlauf der Biegemomente bei identischer statischer und dynamischer Durchbiegung die gleiche Beanspruchung des Armierungsstahles erreicht werden konnte.

\section{1 .4 Zusatzmassen}

Bild 26 zeigt Lage und Anordnung der Zusatzmassen $\Delta M_{1}$ und $\Delta M_{2}$ auf den Prüfbalken (vgl. auch Bild 17).

Bei der Befestigung der Zusatzmassen wurde darauf geachtet, dass die Steifigkeit der Balken nicht verändert wurde. Zudem mussten die aufgebrachten Massen zugfest und spielfrei auf den Balken montiert werden können.

Bild 27 zeigt die verwendeten Bleiplatten zu je $20 \mathrm{~kg}$ mit den Abmessungen $4 \times 22 \times 22 \mathrm{~cm}$. Zwei Stapel zu je 10 Platten konnten auf eine Stahlunterlage mit Pavatex von 10 cm Breite aufgebracht werden. Mit zwei vorgespannten Gewindestangen aus hochwertigem Stahl wurden die Bleimassen auf dem Balken fixiert.

Die Zusatzmassen wurden in zwei Grössen aufgebracht. Die erste Zusatzmasse $\Delta M_{1}$ entsprach einer auf die ganze Balkenlänge bezogenen zusätzlichen, verteilten Last von $152.4 \mathrm{~kg} / \mathrm{m}$. Die zweite Zusatzmasse $\Delta M_{2}$ entsprach einer zusätzlichen, verteilten Last von $295.2 \mathrm{~kg} / \mathrm{m}$.

\section{1 .5 Lager}

Die Lagerung der Versuchsbalken musste folgenden Anforderungen genügen:

- zug- und druckfest

- spielfrei

- zwängungsfrei

- reibungsarm

- seitlich stabil

- Stützweite variabel.

Während der Schwingung konnten wegen der dynamischen Beanspruchung negative Auflagerkräfte auftreten, so dass die Lager zug- und druckfest sein mussten. Die Lager wurden spiel- und zwängungsfrei sowie möglichst reibungsarm ausgebildet, damit Energieverluste innerhalb der Lager, welche die im Versuch feststellbare Dämpfung vergrössern, fast vollständig vermieden werden konnten. Ferner musste die Möglichkeit zur Veränderung der Stützweite vorgesehen werden. 
a) Fistes Lager

Bild 28 zeigt das feste Lager. Die Auflagerdrehwinkel wurden durch zwei Pendelkugellager mit einem Reibungskoeffizienten von unter $2 \%$ ermöglicht. Auf beiden Seiten des Balkens war je eine vertikale, versteifte Stahiplatte angeordnet. Die beiden Platten wurden durch 4 hochwertige Gewindestangen gegeneinander gespannt. Auf die Platten wurde je eine kurze Welle, die den inneren Ring des Kugellagers trug, geschweisst. Die äusseren Ringe der beiden Kugellager sassen in zwei weiteren Stahlplatten, welche die Auflagerkräfte über einen Auflagerbock an den Aufspannboden abgaben.

\section{b) Bewegliches Lager}

Bild 29 zeigt das bewegliche Lager. Es wurde aus Stabilitätsgründen und um eine bessere Zugänglichkeit zum Versuchskörper zu ermöglichen als hängendes Pendellager ausgebildet.

Die Drehung um die Auflagerachse wurde düch eine analoge Konstruktion wie beim festen Lager ermöglicht. Die Längsverschieblichkeit war durch ein aus steifen Stahlplatten hergestelltes Pendel gewährleistet. Am oberen Ende des Pendels wurde ein Bronze-Lager angeordnet, welches sich um eine an einem steifen Rahmen befestigte Welle drehen konnte. Wegen der sehr geringen Drehungen in diesem Lager konnte dort eine etwas grössere Reibung in Kauf genommen werden.

\subsection{Versuchsablauf}

Die Balken wurden in verschiedenen Beanspruchungsstufen geprüft. Zur Kennzeichnung dieser Beanspruchungsstufen wurde ein dreiteiliger Code verwendet, z.B.:

$$
\underbrace{2.5}_{1 .}-\underbrace{0.0}_{2 .}-\underbrace{1}_{\text {3. Code-Zatii }}
$$

Die 1. Code-Zahl steht für die Grösse der maximal zu erreichenden Spannung in der Längsarmierung $\sigma_{\theta}$,abs in Balkenmitte unten, gemessen in $t / \mathrm{cm}^{2}$. Diese Spannung wurde als hauptsächlicher Kennparameter für die Beanspruchungsstufen gewählt. Sie sollte sowohl im. Ausschwing- als auch im Resonanzversuch gerade noch erreicht aber nicht überschritten werden. Aus dem schematischen Beispiel in Bild 30 für die Beanspruchungsstufe 2.5-0.0-1 des Balkens B3 ist diese maximale Stahlspannung sowohl für den Ausschwingversuch als auch für den Resonanzversuch ersichtlich.

Die 2. Code-Zahl gibt die Grösse der Zusatzmasse an. 0.0 bedeutet keine Zusatzmasse, 0.5 steht bei Beanspruchungsstufen mit der Zusatzmasse $\Delta M_{1}, 1.0$ steht bei Beanspruchungsstufen mit der Zusatzmasse $\Delta M_{2}$.

Die 3. Code-Zahl gibt die Prüfphase an, in welcher die Beanspruchungsstufe aufgebracht wurde. 1 steht für die 1. Prüfphase mit einer stützweite von $1=7.88 \mathrm{~m}, 2$ steht für die 2. Prüfphase mit derselben Stützweite von $1=7.88 \mathrm{~m}$, und 3 steht für die 3 . Prüfphase mit der verkürzten Stützweite von $1=4.84 \mathrm{~m}$ und beidseitigen Kragarmen von $1.78 \mathrm{~m}$ Länge.

Das Vorgehen bei der Prüfung eines Balkens soll im folgenden Beispiel des Balkens LB2 anhand der Tabelle 7 mit den numerierten Kolonnen erläutert werden.

In der 1. Prüfphase (1. Kolonne) wurde mit der Beanspruchungsstufe 1.5-0.0-1 (2. Kolonne) begonnen. Diese Beanspruchungsstufe ist durch eine maximal zu erreichende absolute Stahlspannung $\sigma_{e}$, abs von $1.5 \mathrm{t} / \mathrm{cm}^{2}$ (3. Kolonne) und fehlende Zusatzmasse $\Delta M$ (4. Kolonne) gekennzeichnet. Bei einer totalen Masse des Balkens von $179 \mathrm{~kg}$ pro Laufmeter (5. Kolonne) und einer Stützweite I von $7.88 \mathrm{~m}$ (6. Kolonne) ergab sich in der Ruhelage unter zusätz- 
licher Berücksichtigung der Masse des Schwingungserregers ein Moment $M_{0}$ von $1.88 \mathrm{mt}$ (7. Kolonne), welchem eine Stahlspannung $\sigma_{e, 0}$ von $1.31 \mathrm{t} / \mathrm{cm}^{2}$ ( 8 . Kolonne) entsprach. Mit der in dieser Beanspruchungsstufe maximal zu erreichenden absoluten Stahlspannung $\sigma_{e}$, abs von $1.5 \mathrm{t} / \mathrm{cm}^{2}$ konnte auf einfache Weise das bei dynamischer Belastung maximale zusätzliche Moment $M_{\text {rel }}$ von $0.27 \mathrm{mt}$ (9. Kolonne) resp. die maximale zusätzliche Stahlspannung $\sigma_{e, r e l}$ von $0.19 \mathrm{t} / \mathrm{cm}^{2}$ (10. Kolonne) berechnet werden. Beim Ausschwingversuch erforderten die in den 9. und 10. Kolonnen angegebenen Werte eine durch die Spannschraube aufzubringende Kraft $P_{A}$ von $137 \mathrm{kp}$ (11. Kolonne) und eine entsprechende Durchbiegung $\delta_{\max }$, rel von $0.32 \mathrm{~cm}$ (12. Kolonne). Für den Resonanzversuch war eine maximale zusätzliche-Durchbiegung $\delta_{\max , r e l}$ von $0.44 \mathrm{~cm}$ (13. Kolonne) zu erzeugen, damit bei der zu erwartenden Eigenfrequenz $f$ von $3.40 \mathrm{~Hz}$ (14. Kolonne) die in der 9. und 10. Kolonne angegebene Zusatzbeanspruchung erreicht werden konnte.

Nach der Durchführung dieser ersten Beanspruchungsstufe 1.5-0.0-1 wurde die Prüfung mit der Beanspruchungsstufe 2.0-0.0-1 fortgesetzt. Diese Beanspruchungsstufe ist wiederum durch die Angaben in der 1. bis 14. Kolonne der Tabelle 7 festgelegt. Das weitere Prüfprogramm umfasste alle folgenden in Tabelle 7 aufgeführten Beanspruchungsstufen. Die maximale Stahlspannung $\sigma_{\theta}$, abs (3. Kolonne) wurde in Etappen von $0.5 \mathrm{t} / \mathrm{cm}^{2}$ bis nahe an die Streckgrenze der Längsarmierung gesteigert. Bei einer bestimmten Stahlspannung wurde jeweils eine Beanspruchungsstufe ohne Zusatzmassen angeordnet. Beanspruchungsstufen mit den Zusatzmassen $\Delta M_{1}$ und $\Delta M_{2}$ (4. Kolonne). wurden angeordnet, sofern durch diese Zusatzmassen die Stahlspannung $\sigma_{e, 0}$ in Ruhelage ( $\theta$. Kolonne) nicht die durch die Beanspruchungsstufe definierte maximale Stahlspannung $\sigma_{e, a b s}$ (3. Kolonne) überschritt. Bei diesen Beanspruchungsstufen war die zu erreichende maximale Stahlspannung $\sigma_{e}$,abs dieselbe, die Spannungsamplitude $\sigma_{e, r e l}$ war hingegen wegen des erhöhten Eigengewichtes und der zugehörigen Stahlspannung in der Ruhelage $\sigma_{e, 0}$ entsprechend kleiner. Dies ist z.B. für die Beanspruchungsstufen 2.5-0.0-1 und 2.5-0.5-1 aus einem Vergleich der Kolonnen 8 und 10 ersichtlich.

In der 2. Prüfphase wurde dasselbe Prüfprogramm, wie es in der ersten Phase zur Anwendung kam, leicht abgekürzt wiederholt, um den Einfluss der Belastungsgeschichte resp. der Rissbildung - die Risse waren während der 1. Prüfphase entstanden - genauer zu erfassen.

In der 3. Prüfphase wurde die Stützweite der Balken (6. Kolonne) auf $1=4.84 \mathrm{~m}$ verkleinert und ein ähnliches Prüfprogramm wie in der 1. und 2. Prüfphase durchgeführt, allerdings mit Spannungssteigerungen von $1.0 \mathrm{t} / \mathrm{cm}^{2}$ von Beanspruchungsstufe zu Beanspruchungsstufe.

Wegen der erhöhten Stahlspannung in der Ruhelage $\sigma_{e, 0}$ konnten bei den schwächer armierten Versuchsbalken gegenüber den stärker armierten Versuchsbalken während aller Prüfphasen entsprechend weniger Beanspruchungsstufen angeordnet werden.

Bei den Balken LB2 und B2 konnten aus zeitlichen Gründen die Beanspruchungsstufen mit der Zusatzmasse $\Delta M_{1}$ teilweise nicht durchgeführt werden.

Innerhalb jeder Beanspruchungsstufe wurden die folgenden Versuche durchgeführt:

- Vorerst wurden 3 Ausschwingversuche mit jeweils genau gleich grosser Kraft $P_{A}$ (Tab. 7 , 11. Kolonne) durchgeführt.

- Anschliessend wurden 2 Resonanzversuche durchgeführt. Die Resonanzkurven wurden bei konstant gehaltener Erregerkraft punktweise bestimmt, zuerst mit steigender. und dann mit abnehmender Erregerfrequenz.

Bevor die Resonarizkurven aufgenommen werden konnten, war jedoch ein "Vorversuch" notwendig, bei welchem die Grösse der zu wählenden Erregerkraft bestimmt wurde, damit die 
für die entsprechende Beanspruchungsstufe massgebende zusätzliche Stahlspannung $\sigma_{e, r e l}$ (Tabelle 7, 10. Kolonne) möglichst genau erreicht und nicht überschritten wurde. Dabei wurde folgendermassen vorgegangen: Mit sehr klein gewählter Erregerkraft wurde die Resonanzfrequenz, d.h. die erste Eigenfrequenz des Balkens aufgesucht. Die hier auftretende Stahldehnung resp. die entsprechende Stahlspannung kannte über einen Stahldehnungsgeber, der auf einen Kathodenstrahloszillographen geschaltet war, sofort bestimmt werden. Anschliessend wurde die Erregerkraft bzw. der Kolbenweg des Schwingungserregers so gesteigert, dass die angestrebte Stahlspannung gerade erreicht wurde. Damit ergab sich ein erster Wert der für die beiden Resonanzversuche erforderlichen Erregerkraft.

Häufig bildeten sich in den Prüfbalken während der Bestimmung der Erregerkraft neue Risse aus. Dadurch sank die Steifigkeit und damit die Eigenfrequenz (vgl. Gl. (25)) des betreffenden Balkens, worauf die Erregerkraft von neuem bestimmt werden musste. Damit die Ausbildung neuer Risse während der eigentlichen Resonanzversuche möglichst vermieden werden konnte, wurden die Versuchsbalken nach der ersten Bestimmung der benötigten Erregerkraft noch eine Minute lang in der Resonanzschwingung belassen. Auf diese Weise wurde den Balken sozusagen Gelegenheit gegeben, die der Beanspruchungsstufe entsprechenden Risse auszubilden. Erst anschliessend an diesen Vorversuch konnten mit der so bestimmten Erregerkraft die beiden Resonanzkurven aufgenammen werden.

- Zum Schluss wurden nochmals 2 Ausschwingversuche durchgeführt.

Bild 32 zeigt als Beispiel einen Ausschnitt aus dem Prüfprogramm des Balkens LB3.

4. 3 Messungen

Eine ausführliche Beschreibung der Mess- und Registriereinrichtung ist im Anhang 1 angegeben.

Zur Erfassung und Registrierung der Messgrössen standen nebst den üblichen erforderlichen Apparaturen und Geräten folgende Einrichtungen zur Verfügung:

\section{Messgeber:}

- induktive Weggeber für verschiedene Wegamplitudenbereiche

- Beschleunigungsaufnehmer

- Kraftmessdosen

- Dreigelenk-Dehnungsmessrahmen mit einer Messstrecke von $20 \mathrm{~cm}$ Länge zur Aufnahme von Stahl- und Betondehnungen.

\section{Registriergeräte:}

- $x$-t-Schreiber mit 2 Kanälen

- Magnetband mit 4 Kanälen

- Messanlage für statische Versuche, die durch einen Spitzenwertmesser erweitert wurde

- Kathodenstrahloszillograph mit Speicher.

Bild 32 zeigt den für alle Balken gültigen Messstellenplan.

Auf dem $\underline{x-t-S c h r e i b e r}$ ( 2 Kanäle) wurden die

- Durchbiegung $\delta$ in Balkenmitte

- Stahldehnung $\varepsilon_{e}$ der Längsarmierung auf der Balkenunterseite in Balkenmitte aufgenommen. 
Auf dem Magnetband ( 4 Kanäle) wurden die folgenden Messstellen direkt in Funktion der Zeit aufgezeichnet:

- Weg der Schwingmasse $\bar{m}$ resp. Weg des Kolbens a im Zug-Druck-Zylinder des Schwingungserregers

- Durchbiegung $\delta$ in Balkenmitte

- Stahldehnung $\varepsilon_{e}$ der Längsarmierung auf der Balkenunterseite in Balkenmitte

- Betondehnung $\varepsilon_{b}$ auf der Balkenoberseite in Balkenmitte.

An der statischen Messanlage, welche durch einen Spitzenwertmesser erweitert wurde, wurden alle übrigen Messstellen, d.h.

- die Durchbiegungen $\delta$ und $\bar{\delta}$ in allen Messquerschnitten

- die Stahldehnungen $\varepsilon_{e}$ in allen Messquerschnitten auf der Balkenunterseite und der Balkenoberseite

- die Betandehnungen $\varepsilon_{b}$ in allen Messquerschnitten auf der Balkenoberseite und der Balkenunterseite

- die Beschleunigung $b$ in Balkenmitte

angeschlossen. Die gemessenen Daten werden auf einem Lochstreifen festgehalten. Die Messwerte konnten nur nacheinander abgefragt werden. Deshalb wurde diese Anlage nur bei den Resonanzversuchen eingesetzt, wo über längere Zeit stationäre Schwingungen aufrecht erhalten werden konnten.

Auf dem Kathodenstrahloszillographen konnte die

- Stahldehnung $\varepsilon_{e}$ der Längsarmierung auf der Balkenunterseite in Balkenmitte abgelesen werden. Dieser Wert wurde zur Steuerung des Versuchsablaufes verwendet.

\subsection{Auswertungen}

Für die Berechnung der Stahldehnungen bzw. der Stahlspannungen wurde ein Verbundkoeffizient

$$
\kappa=\frac{\varepsilon_{\varepsilon, m}}{\varepsilon_{\varepsilon, \max }}=1.0
$$

angenommen, d.h. die mittlere, über eine Messstrecke von $20 \mathrm{~cm}$ Länge gemessene Stahldehnung und die maximale, im Riss auftretende Stahldehnung werden als gleich gross angenommen.

\subsubsection{Auswertung von Hand}

Die mit Hilfe des $x$-t-Schreibers registrierten Messwerte wurden ausschliesslich von Hand analysiert.

- Ausschwingversuche:

In Bild 33 sind als Beispiel die Resultate von zwei Ausschwingversuchen des Balkens LB3 dargestellt, die zu zwei verschiedenen Beanspruchungsstufen gehören.

Die durch Ausmessen der Kurven ermittelten Werte für

- die maximale relative Durchbiegung $\delta_{\max }$,rel in Balkenmitte

- die maximale relative Stahldehnung $\varepsilon_{e, r e l}$

- die Eigenfrequenz $f$

- das logarithmische Dekrement v 
sind in den einzelnen Figuren angegeben. Die Eigenfrequenz und die Dämpfung wurden jeweils über 10 aufeinanderfolgende Perioden ermittelt. Die fünf pro Beanspruchungsstufe durchgeführten Ausschwingversuche wurden einzeln ausgewertet und die gewonnenen Werte gemittelt.

\section{- Resonanzversuche:}

Die während der Resonanzversuche aufgezeichneten Messignale für

- die maximale relative Durchbiegung $\delta_{\max , r e l}$ in Balkenmitte

- die maximale relative Stahldehnung $\varepsilon_{e, r e l}$

- die Eigenfrequenz $f$

wurden auf analoge Weise ausgewertet.

\subsubsection{Auswertung durch Prozessrechner}

Die Magnetbandaufzeichnungen und die über die erweiterte statische Messanlage auf einem Lochstreifen festgehaltenen Messdaten wurden auch mit Hilfe eines Prozessrechners ausgewertet.

\section{Die auf den vier Kanälen des Magnetbandes aufgezeichneten Messignale}

- Kolbenhub a

- Durchbiegung $\delta$ in Balkenmitte

- Stahldehnung $\varepsilon_{\mathrm{B}}$ der Längsarmierung auf der Balkenunterseite in Balkenmitte und

- Betondehnung $\varepsilon_{b}$ auf der Balkenoberseite in Balkenmitte

wurden über einen PDP-11/45 Computer ausgewertet. Vorerst wurden die vier analogen Signale gleichzeitig mittels eines Analog-Digital-Wandlers mit der gewünschten Abtastfrequenz digitalisiert. Anschliessend wurden die so erhaltenen diskreten Daten für jede Beanspruchungsstufe getrennt nach Ausschwing- und Resonanzversuch weiterverarbeitet.

\section{- Ausschwingversuche:}

Anhand der digitalisierten Daten wurden durch ein Computerprogramm die Eigenfrequenz und die Dämpfung jeweils über 10 sich folgende Perioden ermittelt. Die fünf pro Beanspruchungsstufe durchgeführten Ausschwingversuche wurdeń einzeln ausgewertet und die gewonnenen Werte gemittelt.

\section{- Resonanzversuche:}

Aehnlich wie bei der punktweisen Bestimmung der Resonanzkurven (vgl. Abschnitt 4.2), wurde auch bei der Auswertung punktweise vorgegangen. Bild 34 zeigt als Beispiel die durch den Computer ausgewerteten, zur Beanspruchungsstufe 4.5-0.0-2 des Balkens LB2 gehörigen Resonanzkurven. Aus dem bei einer bestimmten eingestellten Erregerfrequenz erreichten Verlauf der Durchbiegung $\delta_{r e l}$, der Stahldehnung $\varepsilon_{e, r e l}$ und der Betondehnung $\varepsilon_{b, r e l}$ wurden durch das Auswerteprogramm, unter Berücksichtigung der Eichwerte, die extremalen. Werte und die Frequenz bestimmt. Durch ein Zeichnungsprogramm wurden die für jede eingestellte Erregerfrequenz ermittelten Amplituden und Frequenzen in die in Bild 34 angegebenen Diagramme eingetragen. Die Erregerkraft wurde aus dem Kolbenhub a gemäss Gl. (29) ermittelt.

Die mit Hilfe der durch einen Spitzenwertmesser erweiterten statischen Messanlage vor und während der Resonanzversuche registrierten Messdaten

- Durchbiegung $\delta_{r e l}$ und $\bar{\delta}_{r e l}$ in allen Messquerschnitten

- Stahldehnung $\varepsilon_{e, r e l}$ in allen Messquerschnitten auf der Balkenunterseite und der Balkenoberseite

- Betondehnung $\varepsilon_{b, r e l}$ in allen Messquerschnitten auf der Balkenoberseite und der Balkenunterseite

- Beschleunigung b in Balkenmitte 
wurden ebenfalls durch ein Computerprogramm ausgewertet. Aus den Messwerten in Ruhelage und den während der Versuche gemessenen Werten wurden, unter Berücksichtigung der Eichwerte, die maximalen Schwingungsamplituden berechnst. 
In diesem Kapitel werden die an den vier Leichtbeton- und den drei Betónbalken ermittelten Versuchsresultate wiedergegeben.

Erwartungsgemäss ergab sich bei mehreren im gleichen Querschnitt angeordneten Durchbiegungs- resp. Dehnungsaufnehmern und unabhängiger Messung nur selten eine exakte Uebereinstimmung. Während die Werte von an gleicher Stelle mit verschiedenen Aufnehmern gemessenen Durchbiegungen immer sehr gut übereinstimmten, zeigten sich bei den Dehnungsmessungen oft leicht voneinander abweichende Ergebnisse, was insbesondere durch den Einfluss der Risse bedingt war. Zur Darstellung der Resultate wurde deshalb meistens die Durchbiegung gewählt, da diese stets am zuverlässigsten ermittelt werden konnte.

Die in Ruhelage gemessenen Werte werden im Folgenden mit dem tiefgestellten Index "o" ( = Ruhelage) versehen.

Die Differenz zwischen den im Ausschwing- resp. Resonanzversuch und den in der Ruhelage gemessenen Werten wird durch den Index "rel" (= relativ) verdeutlicht.

Der Index "abs" (= absolut) gibt an, dass sich der angegebene Wert auf die im Ausschwingresp. Resonanzversuch gemessenen Werte inkl. den in der Ruhelage gemessenen Werte bezieht.

Ein hochgestellter Index deutet an, ob der angegebene Wert bei einer Durchbiegung des Balkens aus der Ruhelage nach unten (+) oder nach oben (-) ermittelt wurde. Werte ohne hochgestellten Index beziehen sich stets auf eine Durchbiegung des Balkens nach unten.

In den Figuren und Graphiken werden die gemessenen Werte durch einen kleinen Kreis dargestellt.

Wo notwendig, wird jeweils am Schluss eines Abschnittes auf spezielle Unterschiede zwischen den sich entsprechenden Leichtbeton- und Betonbalken hingewiesen bzw. deren Eigenschaften und Verhalten verglichen.

Diese dem Vergleich zwischen Leichtbeton und Beton dienenden Textteile sind links mit einem vertikalen Strich versehen worden.

Derjenige Leser, welcher sich vor allem für den Vergleich interessiert, kann sich.vorwiegend auf die derart hervorgehobenen Stellen konzentrieren.

\subsection{Durchbiegungen und Steifigkeiten}

In Tabelle 8 sind die Durchbiegungen $\delta_{0}$ in Ruhelage für sämtliche versuchsbalken vor und nach Durchführung jeder Beanspruchungsstufe angegeben.

Sofern der Unterschied zwischen den Werten für die Durchbiegung zu. Beginn und nach Abschluss jeder Beanspruchungsstufe klein ist, kann angenommen werden, dass sich in der betreffenden Beanspruchungsstufe keine neuen Risse ausgebildet haben. Bei grösseren Unterschieden ist das Auftreten van neuen Rissen in der zugehörigen Beanspruchungsstufe wahrscheinlich. 


\section{1 .1 Bei statischer Belastung}

In Bild 35 sind die bei den Ausschwingversuchen ohne Zusatzmasse durch die Spannschraube aufgebrachten Kräfte $P_{A}$ in Funktion der dabei aufgezwungenen maximalen relativen Durchbiegung $\delta_{\text {max,rel }}$ in Balkenmitte für sämtliche Versuchsbalken und alle drei Prüfphasen aufgetragen.

Während der 1. Prüfphase bildeten sich bei den anfänglich jeweils nur schwach gerissenen Balken mit zunehmender relativer Durchbiegung ind Beanspruchung weitere Risse aus. Dadurch nahm die Steifigkeit ab, was zum dargesteliten gekrümmten Verlauf der Last-Durchbiegungs-Beziehungen führte. In der 2. und 3. Prüfphase, nachdem die Risse grösstenteils schon in der 1. Prüfphase entstanden waren, verliefen die Last-Durchbiegungs-Beziehungen praktisch linear. Teilweise zeigte sich mit zunehmender Durchbiegung sogar eine gewisse Versteifung.

Alle Balken wiesen auch mit den aufgebrachten Zusatzmassen gegenüber dem Zustand ohne Zusatzmassen ein nahezu identisches Last-Durchbiegungs-Verhalten auf. Wie erwartet, wurden die Prüfbalken durch die für die Befestigung der Zusatzmassen verwendete Vorrichtung praktisch nicht versteift.

Die Leichtbetonbalken benötigten während der 1. und 2. Prüfphase im ganzen untersuchten Beanspruchungsbereich bei gleicher Durchbiegung nur eine geringfügig kleinere Kraft $P_{A}$ als die entsprechenden Betonbalken. In der 3. Prüfphase war die kraft jedoch bei den Leichtbetonbalken etwas höher. Dies kann durch die unterschiedliche Verteilung der Risse resp. Balkenbiegesteifigkeit EI längs der Balkenachsen erklärt werden. Die Leichtbetonbalken waren im mittleren gerissenen Bereich etwas steifer, in den ungerissenen Bereichen gegen die Balkenenden zu wegen des kleineren E-Moduls des Leichtbetons jedoch erheblich weicher als die entsprechenden Betonbalken. In der 1. und 2. Prüfphase überwog der zweite Effekt, in der 3. Prüfphase (verkürzte Stützweite) hingegen der erste.

In Bild 36 ist die aus den Resultaten von Bild 35 berechnete Balkensteifigkeit $k$ ex, bezogen auf die Steifigkeit im ungerissenen Zustand kunger' in Funktion der bei den Ausschwingversuchen aufgezwungenen maximalen Durchbiegung in.Balkenmitte $\delta_{\text {max, rel }}$ aufgetragen.

Als Mass für die vorhandene Steifigkeit $k_{e x}$ wurde das Verhältnis der gemessenen Kraft $P_{A}$ zur dabei gemessenen Durchbiegung $\delta_{\text {max, rel }}$ eingesetzt. Verbindet man in Bild 35 einen beliebigen Punkt einer Kurve mit dem Koordinatenursprung, ergibt die so erhaltene Sekantenneigung ein Mass für die mittlere Steifigkeit des entsprechenden Balkens zum betreffenden Prüfungszeitpunkt.

Diese Sekantensteifigkeit

$$
k_{e x}=\frac{P_{A}}{\delta_{\text {max }, r e l}}
$$

wurde in Bild 36 auf den im ungerissenen Zustand zu erwartenden Steifigkeitswert k unger bezogen. Dieser wurde aus der Beziehung

$$
k_{\text {unger }}=\frac{48 \cdot(E I) \text { unger }}{1^{3}}
$$

berechnet, wabei für EI die Werte van Tabelle 5 eingesetzt wurden.

Es können die folgenden Feststellungen gemacht werden:

- Während der Rissbildung (1. Prüfphase) nahm die Steifigkeit stark ab. 
- Sobald annähernd alle Biegerisse ausgebildet waren, blieb die Steifigkeit praktisch konstant (2. Prüfphase).

- In der 3. Prüfphase, sowie andeutungsweise auch schon in der 2. Prüfphase, nahm bei fast allen Balken die Steifigkeit mit zunehmender Beanspruchung leicht zu (überlineares Verhalten). Nur bei den Balken LB1 und 81 fiel die Steifigkeit zuerst noch beträchtlich ab, da sich bei diesen relativ schwach armierten Balken vor allem in den Bereichen über den Auflagern auch in der 3. Prüfphase noch weitere Risse bildeten.

- Erwartungsgemäss nahm bei allen Balken die bezogene Steifigkeit $k_{\text {ex }} / k_{\text {unger }}$ mit abnehmendem Armierungsgehalt ab.

- Bei den Leichtbetanbalken nahm die bezogene Steifigkeit $k_{\text {ex }} / k_{\text {unger }}$ mit fortschreitender Ausbildung der Risse wesentlich weniger ab als bei den Betonbalken.

- Der maximale Steifigkeitsabfall gegenüber dem ungerissenen Zustand betrug in der 2. Prüfphase bei den Leichtbetonbalken 55 bis $68 \%$, bei den Betonbalken 71 bis $79 \%$. In der 3. Prüfphase fiel die Steifigkeit bei den Leichtbetonbalken um 57 bis $71 \%$, bei den Betonbalken um 68 bis $84 \%$.

Die Bilder 37a bzw. 37b zeigen die nach der 1. bzw. 2. Prüfphase mit Hilfe der separat angeordneten, nur für statische Prüfung geeigneten Belastungsvorrichtung (vgl. Bild 25) aufgenommenen Last-Durchbiegungs-Diagramme aller Versuchsbalken.

Auf der Ordinate ist die statische Last $P_{\text {statisch }}$ angegeben. Auf der Abszisse ist die relative Durchbiegung in Balkenmitte $\delta_{r e l}$ infolge dieser Last $P_{\text {statisch }}$ aufgetragen. Das Last-Durchbiegungs-Verhalten wurde nach mehreren Beanspruchungsstufen der 1. und 2. Prüfphase bestimmt. Dabei wurde jedes Mal die Last-Durchbiegungs-Charakteristik im positiven Bereich (Belastung nach unten gerichtet, Durchbiegung nach unten) und im negativen Bereich (Belastung nach oben gerichtet, Durchbiegung nach oben) aufgenommen.

Die gemessenen Werte bestätigten den in den Bildern $35 \mathrm{bzw}$. 36 angegebenen Verlauf der Steifigkeiten. Auch hier nahm zum Teil die Kraft leicht überlinear zu, d.h. die Steifigkeit nahm mit grösser werdender Durchbiegung zu.

Eine Möglichkeit zur Erklärung dieser interessanten und eher ungewöhnlichen Feststellung ist die folgende:

Das Bild 37c zeigt ein Risselement eines Stahlbetonbalkens. Unterhalb des Risselementes ist der zu zwei verschieden hohen Biegebeanspruchungen gehörige Verlauf der Stahldehnungen $\varepsilon_{e}$ angegeben. 'Die maximale Stahldehnung $\varepsilon_{e \text {,max }}$ tritt jeweils im Riss auf, während die Stahldehnung zwischen zwei Rissen infolge der Wirkung des Verbundes zwischen Stahl und Beton wesentlich kleiner sein kann. Das Verhältnis $k$ der mittleren Stahldehnung $\varepsilon_{e, m}$ (in Bild 37c)gestrichelt eingezeichnet) zur maximalen Stahldehnung $\varepsilon_{e, m a x}$ im Riss

$$
k=\frac{\varepsilon_{e, m}}{\varepsilon_{e, \text { max }}}
$$

beeinflusst erheblich die Biegesteifigkeit des Risselementes. Bei grossen Biegebeanspruchungen resp. grossen Stahldehnungen kann der Koeffizient k kleiner sein als bei kleinen Biegebeanspruchungen.

Die Biegesteifigkeit EI lässt sich aus dem Biegemoment M und der mittleren Querschnittskrümmung $\Phi_{m}$ aus der Gleichung

$$
E I=\frac{M}{\Phi_{m}}
$$


berechnen.

Mit

$$
\begin{aligned}
& \Phi_{m}=\frac{\varepsilon_{\theta, m}}{h-x} \quad \text { und } \\
& M=z \cdot y=F_{e} \cdot \sigma_{\varepsilon, \text { max }} \cdot y=F_{e} \cdot E_{\theta} \cdot \varepsilon_{\theta, \text { max }} \cdot y
\end{aligned}
$$

erhält man EI zu

$$
E I=E_{e} \cdot F_{e} \cdot y \cdot(h-x) \cdot \frac{\varepsilon_{e, \max }}{\varepsilon_{e, m}}=E_{e} \cdot F_{e} \cdot y \cdot(h-x) \cdot \frac{1}{k}
$$

Mit kleinerem $k$ wird somit die Biegesteifigkeit EI grösser.

Normalerweise wird dieses Verhalten bei üblichen Versuchen ohne grässere Lastwechselzahlen nicht beobachtet. Der Grund dazu dürfte darin liegen, dass bei solchen Versuchen das Anfangskriechen in jeder Laststufe bedeutend ist und dadurch die jeweilige steifigkeit reduziert wird. Bei den hier durchgeführten Versuchen hat das Anfangskriechen wegen der vielen durchgeführten Lastwechsel praktisch keinen Einfluss mehr.

Die festgestellte Vergrösserung der Steifigkeit mit zunehmender Beanspruchungshöhe wurde bei den zugehörigen Beanspruchungsstufen auch durch ansteigende Eigenfrequenzen ( $v g$ l. Bild 53) und durch nach rechts kippende Resonanzkurven, die nur bei überlinearem Verhalten auftreten können, dokumentiert ( $v g l$. Bilder 8 und $38 \mathrm{c}$ ).

\subsubsection{Bei dynamischer Belastung}

In Tabelle 9 sind die während der Resonanzversuche in jeder Beanspruchungsstufe aufgebrachten Erregerkräfte $P_{E \Gamma r}$ zusammen mit den jeweils erreichten maximalen Durchbiegungen $\delta_{\text {max,rel }}$ für alle Balken und alle durchgeführten Beanspruchungsstufen angegeben. Die Erregerkraft $P_{\text {Err }}$ wurde aus G1. (29) berechnet.

Bei den Leichtbetonbalken war zum Erreichen einer bestimmten relativen Durchbiegung stets eine geringere Erregerkraft erforderlich als bei den entsprechenden Betonbalken. Die geringsten Erregerkräfte wurden jeweils für den Balken LB4 benötigt.

Die Bilder 38a, b und c zeigen einige ausgewählte Resonanzkurven, stellvertretend für die zahlreichen ausgewerteten Resonanzversuche. In jeweils vier Darstellungen sind die gemäss Gl. (29) berechnete Erregerkraft $P_{E \Gamma \Gamma}$, die relative Durchbiegung $\delta_{\text {rel }}$, die relative Stahldehnung an der Balkenunterseite $\varepsilon_{e}$, rel sowie die relative Betondehnung $\varepsilon_{b}, r e l$ an der Balkenoberseite - alle in Balkenmitte - in Funktion der Erregerfrequenz $\Omega$ aufgetragen.

In Bild 38a sind die zur Beanspruchungsstufe 4.5-0.0-2 des Balkens B1 gehörigen Resonanzkurven dargestellt. Die Balkensteifigkeit war in der 2. Prüfphase praktisch konstant (lineares Verhalten, vgl. Bild 35). Daraus resultierte eine praktisch vertikale Resonanzkurve.

Bild 38b zeigt eine nach links kippende Resonanzkurve bei leicht unterlinearem Verhalten (unterlineare Balkensteifigkeit, in Bild 35 nicht dargestellt) und zwar beim Balken B3 für die Beanspruchungsstufe 3.5-1.0-2.

In Bild 38c ist eine nach rechts kippende Resonanzkurve bei überlinearem Verhalten (überlineare Balkensteifigkeit, vgl. Bild 35) und zwar beim Balken LB3 und Beanspruchungsstufe 3.5-0.0-3 dargestellt. 
Aus Bild 39 ist das Verhältnis der relativen Durchbiegungen $\bar{\delta}_{\text {max, rel }}(2.60 \mathrm{~m}$ von den Balkenenden entfernt gemessen) $z u \delta_{\max , r e l}$ (in Balkenmitte gemessen), die während der 2. und 3. Prüfphase mit Hilfe der in den entsprechenden Messquerschnitten angeordneten Aufnehmern (vgl. 32) gemessen wurden, in Abhängigkeit von der maximalen relativen Durchbiegung $\delta_{\text {max, rel }}$ ersichtlich.

Mit den angegebenen Durchbiegungsverhältnissen $\bar{\delta}_{\max , \text { rel }} / \delta_{\max , \text { rel }}$ kann die form der relativen Biegelinie bei jeder Beanspruchungsstufe näherungsweise bestimmt werden. Bei der 2. Prüfphase, sowie auch bei der nicht dargestellten 1. Prüfphase, entsprach die ermittelte Form der relativen Biegelinie im ganzen Beanspruchungsbereich einem annähernd sinusförmigen Verlauf der relativen Durchbiegungen längs der Balkenachse, wie es auch theoretisch für einen elastischen Balken mit gleichmässig verteilter Masse m und Biegesteifigkeit EI zu erwarten war:

$$
\begin{aligned}
\bar{\delta}_{r e l}=\delta_{r e l} \cdot \sin (\Omega t) \cdot \sin \left(\frac{\pi \cdot 2.34}{7.88}\right) & =0.803 \cdot \delta_{r e l} \cdot \sin (\Omega t) \\
\delta_{\max , r e l}= & =0.803 \cdot \delta_{\max , r e l}
\end{aligned}
$$

In der 3. Prüfphase, also bei reduzierter Stützweite der Balken, variierte das Verhältnis zwischen der Durchbiegung am Kragarmende $\bar{\delta}_{\max , \text { rel }}$ und der Durchbiegung in Balkenmitte $\delta_{\max , \mathrm{rel}}$ zwischen $\delta_{\max , \mathrm{rel}}=1.15 \cdot \delta_{\max , \mathrm{rel}}$ und $\delta_{\max , \mathrm{rel}}=1.31 \cdot \delta_{\max , \mathrm{rel}}$. Für gleichmässig verteilte Masse $m$ und Biegesteifigkeit EI würde sich theoretisch ein wert von $\bar{\delta}_{\max , r e l}=1.34 \cdot \delta_{\max , r e l}$ ergeben.

In Bild 40 ist für die 2. und 3. Prüfphase das Verhältnis zwischen den negativen relativen Durchbiegungen (Durchbiegung nach oben) $\delta_{\text {max, rel }}^{-}$und den zeitlich darauffolgenden

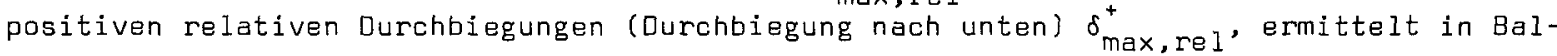
kenmitte bei Resonanz, in Funktion der maximalen relativen Durchbiegung in Balkenmitte $\delta_{\text {max,rel }}$ wiedergegeben.

In der 2. Prüfphase, sowie auch bei der hier nicht dargestellten 1. Prüfphase, waren bei kleinen relativen Durchbiegungen die positiven und die negativen Durchbiegungen noch etwa gleich gross. Bei grösseren Durchbiegungen nahmen die negativen Durchbiegungen stärker zu als die positiven. Diese Zunahme war vor allem bei den Betonbalken sehr ausgeprägt.

Hätten die Versuchsbalken im Zug- und Druckbereich eine identische Steifigkeitscharakteristik aufgewiesen, hätten theoretisch betrachtet die positiven und negativen Durchbiegungen gleich gross sein sollen. Der unregelmässige Verlauf der dargestellten Durchbiegungsverhältnisse $\delta_{\max , r e l}^{-} / \delta_{\max }^{+}$, rel resultierte somit aus dem unterschiedlichen Steifigkeitsverhalten bei Durchbiegung nach oben und unten (vgl. Bild 37).

In der 3. Prüfphase waren die Unterschiede zwischen positiven und negativen relativen Durchbiegungen bei kleinen Durchbiegungen zum Teil beträchtlich. Bei grösseren relativen Durchbiegungen wurden die positiven und negativen Durchbiegungen wieder etwa gleich gross.

In der 3. Prüfphase trat wegen der verkürzten Stützweite der Einfluss des Verformungszustandes in der Ruhelage gegenüber demjenigen der dynamischen Beanspruchung weit zurück. Die oberen und unteren Randfasern erfuhren während eines Schwingzyklus vor allem bei grossen Durchbiegungen annähernd gleich grosse absolute Zug- resp. Druckbeanspruchungen. Damit wiesen die Prüfkörper während der 3. Prüfphase im positiven und negativen Durchbiegungsbereich ein ähnliches Last- und Durchbiegungsverhalten auf. Dies führte zu annähernd gleich grossen positiven und negativen Durchbiegungen. 


\subsection{Beschleunigungen}

Bei einer zeitlich sinusförmig verlaufenden Erregerkraft ergibt sich ein sinusförmiger Verlauf der Durchbiegungen

$$
\delta(t)=\delta_{\max , r e 1} \cdot \sin (\Omega t)
$$

Daraus kann der Verlauf der Beschleunigungen zú

$$
b(t)=-\delta_{\max , \Gamma e 1} \cdot \Omega^{2} \cdot \sin (\Omega t)
$$

berechnet werden. Bei Resonanz ergibt sich mit $\omega=\Omega$

$$
\begin{aligned}
& b(t)=-\delta_{\max , r e l} \cdot \frac{k}{m} \cdot \sin (\Omega t) \\
& b_{\text {max }}=-\delta_{\max , r e l} \cdot \frac{k}{m}
\end{aligned}
$$

In Bild 41a sind die in Balkenmitte bei Resonanz gemessenen maximalen Beschleunigungen $b_{\text {max }}$ (für Durchbiegung nach unten) für sämtliche Versuchsbalken und alle drei Prüfphasen in Abhängigkeit von der bei Resonanz erzielten maximalen relativen Durchbiegung $\delta_{\max , \text { rel }}$ aufgetragen.

In der 1. Prüfphase ist der Einfluss der Rissbildung sehr stark sichtbar. Die Beschleunigung nahm langsamer zu als die relative Durchbiegung.

In der 2. Prüfphase nahm die Beschleunigung praktisch linear mit der Durchbiegung zu.

In der 3. Prüfphase nahm die Beschleunigung der stärker armierten Balken leicht überproportional zu, während sie bei den schwach bewehrten Balken nur unterproportional zunahm.

G1. (36) zeigt, dass die Beschleunigung im wesentlichen durch die maximale relative Durchbiegung und die steifigkeit bestimnt wird. Dies wird auch durch einen Vergleich der Bilder 36 und 41 a bestätigt. Je nach der Grösse der Steifigkeit änderte sich der Verlauf der Beschleunigungs-Durchbiegungs-Beziehung in Bild $41 \mathrm{a}$.

In Bild 41b sind die gemessenen und die nach Gl. (36) und mit den in Bild 35 angegebenen Steifigkeiten berechneten maximalen Beschleunigungen $b_{\max }$ des Balkens LB3 - stellvertretend für alle Balken - in Funktion der maximal erreichten relativen Durchbiegungen $\delta_{\text {max,rel }}$ aufgetragen. Die Uebereinstimmung von Versuch und Rechnung ist befriedigend. Daraus kann u.a. geschlossen werden, dass die Bewegung der Prüfbalken wie angestrebt zeitlich etwa sinusförmig verlief, da die Gl. (36) nur für einen sinusförmigen Durchbiegungsverlauf Gültigkeit hat. Grössere Abweichungen traten jeweils erst bei grösseren Durchbiegungen auf.

Es können die folgenden Feststellungen gemacht werden:

- Die stärker armierten Balken wiesen entsprechend ihrer höheren Steifigkeit (vgl. Bild 36) resp. Eigenfrequenz ( $v g l$. Bild 54 ) bei gleich grossen Durchbiegungen $\delta_{\max , \text { rel }}$ in allen Prüfphasen grössere maximale Beschleunigungen $b_{\max }$ auf. 
- Die Beschleunigungen der Leichtbetanbalken waren stets grösser als diejenigen der entsprechenden Betonbalken, da die Leichtbetonbalken bei annähernd gleicher Steifigkeit eine erheblich geringere Masse aufwiesen (vgl. Gl. (36)). Bild 41b z.B. zeigt für die 2. Prüfphase sehr schön diesen mit zunehmender Zusatzmasse abnehmenden Unterschied zwischen Leichtbeton- und Betonbalken.

\subsection{Biegemomente und Querkräfte}

Das Bild 42a zeigt am Beispiel des. Balkens LB3, stellvertretend für alle Balken, den Verlauf der Biegemomente $M_{0}$ und der Querkräfte $Q_{0}$ in Ruhelage und zwar für den Zustand ohne Zusatzmasse sowie mit den Zusatzmassen $\Delta M_{1}$ und $\Delta M_{2}$.

Die Biegemomente und Querkräfte in den Balken infolge der dynamischen Belastung lassen sich aus den gemessenen resp. den aus relativen Durchbiegungen und Eigenfrequenzen berechneten Beschleunigungen sowie unter Berücksichtigung der Form der Biegelinie und der Masse des Balkens, des Schwingers und der Zusatzmassen nach dem Prinzip von d'Alembert ermitteln.

Das Bild 42b zeigt, stellvertretend für alle Prüfbalken, den Verlauf des relativen Biegemomentes $M_{\Gamma e l}$ und der relativen Querkraft $Q_{\text {rel }}$ längs der Stabachse des Balkens LB3. Die angegebenen Werte sind für eine maximale relative Durchbiegung $\delta_{\text {max, rel }}$ van $1.0 \mathrm{~cm}$ und eine Frequenz $f$ von $1.0 \mathrm{~Hz}$ berechmet. Die effektiven Schnittkräfte ergeben sich durch Multiplikation mit dem Faktor $\delta_{\max , r e l} \cdot f^{2}$.

In den Bildern 43a, b und 0 sind die in der 1., 2. und 3. Prüfphase bei Resonanz erreichten maximalen Biegemomente $M_{\max , r e l}$ in Balkenmitte in Funktion der maximalen Durchbiegung $\delta_{\max , r e l}$ angegeben, und zwar sowohl für die Beanspruchungsstufen ohne Zusatzmassen als auch für die Beanspruchungsstufén mit den Zusatzmassen $\Delta M_{1}$ und $\Delta M_{2}$.

Wie bei den Beschleunigungen (Bild 41a) waren die Abweichungen vom linearen Verlauf durch Aenderungen in der Balkensteifigkeit resp. Eigenfrequenz bedingt.

In Bild 44 ist die maximale Querkraft $Q_{\max }, r e l$ beim Auflager in Funktion der maximalen Durchbiegung $\delta_{\text {max,rel }}$ für die 1.,2. und 3. Prüfphase (jeweils ohne Zusatzmasse) angegeben. Die absolute Schubspannung $\tau_{a b s}$ überschritt auch bei grösserer Beanspruchung bei keinem Balken die untere Schubspannungsgrenze.

Die Schnittkräfte infolge der dynamischen Belastung der sich entsprechenden Leichtbeton- und Betonbalken waren stets annähernd gleich gross. Der Grund dafür war, dass sich die Einflüsse der im gerissenen Zustand grösseren Eigenfrequenz und der kleineren

Masse der Leichtbetonbalken weitgehend kompensierten.

\subsection{Dehnungen}

In Bild 45 sind die in Balkenmitte jeweils bei Resonanz an der Balkenunterseite gemessenen maximalen positiven relativen Stahldehnungen $\varepsilon_{e, r e l}$ in Abhängigkeit der zugehörigen maximalen Durchbiegung $\delta_{\text {max, rel }}$ für alle Prüfphasen aufgetragen. Erwartungsgemäss ergab sich bei den Beanspruchungsstufen mit Zusatzmassen jeweils bei gleicher relativer Durchbiegung dieselbe relative Stahldehnung, so dass in Bild 45 nur die Werte der Beanspruchungsstufen ohne Zusatzmassen angegeben sind. 
der relativen Durchbiegung zu.

Die schwach armierten Prüfbalken wiesen bei gleicher relativer Durchbiegung, trotz der geringeren Biegemomente ( $v g l$. Bild 43), stets eine grössere Stahldehnung auf als die stärker bewehrten Balken.

Wie aufgrund des in den Bildern $43 a$, b und $c$ angegebenen Verlaufs der Biegemomente
zu erwarten war, ergaben sich für entsprechende Leichtbeton- und Betonbalken bei
gleicher relativer Durchbiegung ungefähr gleich grosse relative Stahldehnungen.

In Bild 46 sind die in Balkenmitte an der Unterseite gemessenen maximalen negativen relativen Stahldehnungen $\varepsilon_{e}^{-}$,rel (bei Durchbiegung nach oben ermittelt) in Funktion der maximalen negativen Durchbiegung $\delta_{\text {max, rel }}^{-}$in Balkenmitte für alle Prüfphasen, jedoch nur für die Beanspruchungsstufen ohne Zusatzmassen aufgetragen.

Je nach der Höhe der Stahldehnung $\varepsilon_{e, 0}$ in Ruhelage und der Grösse der relativen Stahldehnung $\varepsilon_{e, r e l}^{-}$konnte die resultierende absolute Stahldehnung $\varepsilon_{e, a b s}$ eine Druck- oder Zugspannung sein. Solange die untere Randfaser noch eine Zugbeanspruchung erfuhr $\left(\varepsilon_{e, a b s}>0\right)$ nahmen die negativen Stahldehnungen noch praktisch linear mit der negativen Durchbiegung zu. Sobald jedoch auf der Unterseite Druckspannungen $\left(\varepsilon_{e}\right.$,abs $<0$ ) entstanden, nahm die negative Stahldehnung nur noch unterlinear zu. Je grösser der Armierungsgehalt war, desto früher (d.h. bei kleinerer negativer relativer Durchbiegung) begann der unterlineare Bereich. Wegen der recht hohen Beanspruchung der relativ schwach armierten Balken LB1 und B1 im Ruhezustand, trat bei diesen Balken während der 1. und 2. Prüfphase an der Balkenunterseite praktisch nie Druck auf, womit die Stahldehnungs-Durchbiegungs-Beziehung noch linear blieb. In der 3. Prüfphase erfuhr die untere Randfaser an der Balkenunterseite bei viel kleineren negativen relativen Durchbiegungen eine Druckbeanspruchung, da die Beanspruchung im Ruhezustand wesentich geringer war.

In Bild 47 sind die in Balkenmitte jeweils bei Resonanz an der Oberseite gemessenen maximalen relativen Betonstauchungen $\varepsilon_{b, r e l}$ in Funktion der zugehörigen maximalen Durchbiegung $\delta_{\max , r e l}$ für alle Prüfphasen aufgetragen.

Bei allen Balken nahm die Betonstauchung unabhängig von der Prüfphase nahezu linear mit der Durchbiegung zu. Zwischen den verschiedenen Prüfbalken traten dabei geringe, eher unsystematische Unterschiede auf.

Die Leichtbetonbalken wiesen bei gleicher relativer Durchbiegung ungefähr die gleichen relativen Betonstauchungen auf wie die entsprechenden Betonbalken.

In Bild 48 sind die in Balkenmitte an der Dberseite gemessenen maximalen negativen relativen Betondehnungen $\varepsilon_{b}^{-}, \mathrm{rel}$ in Funktion der maximalen negativen Durchbiegung $\delta_{\text {max, rel }}^{-}$ wiedergegeben. Bei grösseren negativen relativen Biegemomenten, d.h. sobald die Momente infolge Eigengewichtes der Prüfbalken durch die Momente infolge der. Trägheitskräfte kompensiert waren, traten während jedes Schwingungszyklus an der Balkenoberseite Zugspan-

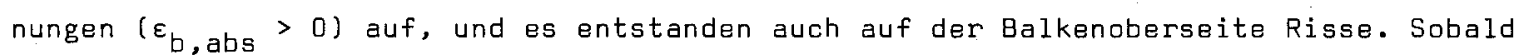
dies der Fall war, nahmen die Betondehnungen mit grösser werdender Durchbiegung stärker zu.

Aus den Stahldehnungen $\varepsilon_{e}$ und Betondehnungen $\varepsilon_{b}$, wie sie in den Bildern 45 und 47 wiedergegeben sind, lässt sich unter der Annahme des Ebenbleibens der Querschnitte die Lage der neutralen Achse, d.h. der Abstand $x$ der neutralen Achse von der Druckkante berechnen. Dieser ist in Bild 49 wiederum in Funktion der maximalen relativen positiven Durchbiegung $\delta_{\max , \text { rel }}$ dargestelit. 
Wie aufgrund der elastischen Theorie zu erwarten war, lag die neutrale Achse bei den Balken mit grösserem Armierungsgehalt tiefer als bei den schwächer bewehrten Balken. Die aus den gemessenen Stahl- und Betondehnungen ermittelte Lage der neutralen Achse stimmte bei höherer Beanspruchung recht gut mit den aufgrund der in Tabelle 5 ángegebenen EModuli berechneten Werten überein. Die fortschreitende Rissbildung während der 1. Prüfphase wurde sehr schön durch die Verringerung des Abstandes der neutralen Achse gezeigt. Unter Berücksichtigung der Lage der neutralen Achse und der Lage und Grösse der Zug- und Druckarmierung kann aus Bild 49 der innere Hebelarm y der Betondruck- und Stahlzugkräfte berechnet werden. Unter Einbezug der in Bild 45 wiedergegebenen Stahldehnungen kann auch das innere Moment

$$
\cdot M_{i, r e 1}=F_{e} \cdot \sigma_{e, r e l} \cdot y=F_{e} \cdot E_{e} \cdot \varepsilon_{e, r e l} \cdot y
$$

ermittelt werden. Der (hier nicht dargestellte) Vergleich des derart erhaltenen inneren Momentes $M_{i, r e l}$ mit dem äusseren Moment $M_{\text {max,rel }}$ aus Bild 43a zeigte für alle Balken eine sehr gute Uebereinstimmung. Die gemessenen Werte der Stahldehnung konnten damit rechnerisch gut bestätigt werden.

Für eine negative Durchbiegung nach oben kann auf gleiche Weise wie dies für eine positive Durchbiegung nach unter in Bild 49 getan wurde, aus den angegebenen negativen Stahlstauchungen $\varepsilon_{e}^{-}$und Betondehnungen $\varepsilon_{b}^{-}$der Bilder 46 und 48 der Abstand $x^{-}$der neutralen Achse von der Druckkante berechnet werden.

In Bild 50 ist der Abstand $x^{-}$der neutralen Achse, vom unteren Balkenrand aus gemessen, wiederum in Funktion der maximalen negativen Durchbiegung $\delta_{\max }^{-}$, rel in Balkenmitte aufgetragen. Mit zunehmender Rissbildung musste hier der Abstand $x^{-}$zuerst grösser werden. Erst wenn die negativen Momente $M_{r e l}^{-}$infolge dynamischer Belastung die positiven Momente $M_{0}$ infolge Eigengewichtes kompensiert hatten, konnte der Abstand $x^{-}$kleiner werden und damit die neutrale Achse absinken. Am ausgeprägtesten war dies bei der 3. Prüfphase und bei den stark armierten Balken LB3 und B3 zu beobachten.

In Bild 51 ist in zu Bild 50 analoger Darstellung der Einfluss der Zusatzmasse $\Delta M$ auf den Abstand $x^{-}$der neutralen Achse von der Druckkante, stellvertretend für alle Balken, anhand des Balkens $B 3$ wiedergegeben. Mit grösserer Zusatzmasse, d.h. grösserem Biegemoment $M_{0}$ in der Ruhelage, waren grössere negative Momente $M_{r e l}^{-}$erforderlich bis die obere Randfaser Zugspannungen erhielt. Deshalb nahm der Abstand $x^{-}$der neutralen Achse bei grösser werdender Zusatzmasse gegenüber dem Zustand ohne Zusatzmasse erst bei grösserer Durchbiegung resp. Beanspruchung ab.

\subsection{Rissverhalten}

In Bild 52 ist die maximale in Balkenmitte an einem Riss gemessene relative Rissöffnung $r_{\max , r e l}$ in Funktion der maximalen positiven Durchbiegung $\delta_{\max , r e l}$ in Balkenmitte angegeben. Bei allen Balken nahm die Rissöffnung praktisch linear mit der Durchbiegung zu. Sie lag bei allen Balken in derselben Grössenordnung und überstieg im untersuchten Beanspruchungsbereich nie den wert von $25 / 100 \mathrm{~mm}$.

Die Rissbilder der einzelnen Prüfbalken nach der ersten Prüfphase sind in Bild 53 abgebildet.

In denjenigen Querschnitten, in welchen die Bügel angeordnet waren (vgl. Bild 18), traten meistens die ersten Risse auf. Sehr häufig öffneten sich bei grösser werdender Beanspruchung auch in den Querschnitten zwischen den Bügeln zusätzliche Risse. 
Bei den Leichtbetonbalken war der mittlere Abstand der einzelnen Risse erwartungsgemäss kleiner als bei den entsprechenden Betonbalken. Die Leichtbetonbalken wiesen demzufolge gegenüber den Betonbalken auch eine grössere Zahl von Rissen auf.

\subsection{Eigenfrequenzen}

In Bild 54 a ist die Grösse der bei den Resonanzversuchen und den Beanspruchungsstufen ohne Zusatzmassen festgestellten ersten Eigenfrequenzen $f$ der Prüfbalken in Funktion der maximalen positiven Durchbiegung $\delta_{\text {max,rel }}$ in Balkenmitte für alle Prüfphasen aufgetragen.

Die aus den Ausschwingversuchen ermittelten Eigenfrequenzen stimmten sehr gut mit denjenigen aus den Resonanzversuchen überein und werden deshalb hier nicht aufgeführt.

Es können die folgenden Feststellungen gemacht werden:

- In der 1. Prüfphase nahm die Eigenfrequenz wegen der fortschreitenden Ausbildung der Risse und des dadurch verursachten Steifigkeitsabfalls (vgl. Bild 36) mit wachsender Durchbiegung resp. Stahldehnung ab.

- Die Abnahme der Eigenfrequenz gegenüber dem ungerissenen Zustand betrug bis zur 2. Prüfphase bei den Leichtbetonbalken 33 bis $44 \%$, bei den Betonbalken 47 bis $54 \%$.

- In der 2. Prüfphase änderte sich die Balkensteifigkeit im allgemeinen nur noch sehr wenig. Deshalb war hier die Eigenfrequenz im ganzen Beanspruchungsbereich praktisch konstant.

- In der 3. Prüfphase (sowie zum Teil auch in der 2. Prüfphase) nahm die Eigenfrequenz bei den stärker armierten Balken wegen der in Bild 36 dargestellten Zunahme der Steifigkeit mit steigender Beanspruchung zu. Bei den schwach armierten Balken LB1 und $B 1$ war auch hier zuerst noch ein erheblicher Abfall der Eigenfrequenz zu beobachten, da deren Steifigkeit durch neu auftretende Risse zu Beginn dieser Prüfphase vermindert worden ist.

- Wie zu erwarten war, wiesen die schwächer armierten Versuchsbalken gegenüber den stärker armierten Balken eine tiefere Eigenfrequenz auf.

Die anfänglich kleinere Eigenfrequenz der Leichtbetonbalken, trotz geringerer Masse durch den wesentlich kleineren É-Modul bedingt, nahm im Vergleich zu den entsprechenden Betonbalken mit zunehmender Beanspruchung und Rissbildung weniger stark ab. Schliesslich wiesen alle Leichtbetonbalken im gerissenen Zustand eine höhere Eigenfrequenz auf als die entsprechenden Betonbalken.

Ein zu den Beanspruchungsstufen ohne Zusatzmassen (vgl. Bild 54a) analoges Verhalten konnte festgestellt werden, wenn die Beanspruchungsstufen mit den Zusatzmassen $\Delta M$ betrachtet wurden. Die Bilder 54b und $54 \mathrm{c}$ zeigen den Verlauf der ersten Eigenfrequenz aller Balken mit den Zusatzmassen $\Delta M_{1}$ und $\Delta M_{2}$. Je grösser die Masse inkl. Zusatzmasse war, desto kleiner wurde die Eigenfrequenz.

Während die Leichtbetonbalken bei den Beanspruchungsstufen ohne Zusatzmasse (Bild 54a) nach Abschluss der Rissbildung eine beträchtlich höhere Eigenfrequenz aufwiesen als die entsprechenden Betonbalken, wurde dieser Unterschied mit zunehmender Zusatzmasse $\Delta M$ erwartungsgemäss (Bild 54b und Bild 54c) immer kleiner. 


\subsection{Dämpfung}

Aus praktischen Gründen und mangels besserer Möglichkeiten wurde bei der Versuchsauswertung als Kenngrösse für die Dämpfung das nur für ein viskoses Dämpfungsgesetz und linear elastisches Verhalten eines Schwingers gültige logarithmische Dekrement $v$ gewählt, unabhängig davon, ob das effektive Dämpfungsverhalten diesem Ansatz genügte oder nicht.

\subsubsection{Bestimmung aus Ausschwingversuchen}

Die Bilder 55a, b und c zeigen für alle Beanspruchungsstufen mit und ohne Zusatzmassen den aus den Ausschwingversuchen aller Beanspruchungsstufen gewonnenen Verlauf des logarithmischen Dekrementes $v$ in Funktion der maximalen positiven Durchbiegung $\delta$ (Anfangsdurchbiegung) in Balkenmitte. Dabei wurde jeweils das logarithmische Dekrement aus den ersten zehn Perioden des Ausschwingversuchs ermittelt (Abschnitt I in Bild 33)

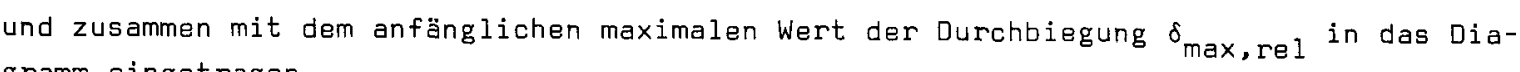
gramm eingetragen.

Verlauf der Dämpfung der einzelnen Balken während des Versuchsablaufs:

Während aller Prüfphasen war die ermittelte Dämpfung stark von der Durchbiegung resp. Beanspruchungshöhe abhängig.

\section{Prüfphase (fortschreitende Ausbildung der Risse):}

In der 1. Prüfphase ohne Zusatzmasse (1. Figur in Bild 55a) nahm die Dämpfung bei sämtlichen Versuchsbalken während der Rissbildung mit grösser werdender relativer Durchbiegung zuerst zu. Mit zunehmender Durchbiegung resp. Beanspruchung nahm dann aber die Dämpfung wieder stark ab. Nach Abschluss der Rissbildung wurden für die Dämpfung bei grossen relativen Durchbiegungen zumị Teil sehr kleine Werte gemessen.

In der 1. Prüfphase mit der Zusatzmasse $\Delta M_{1}$ (1. Figur in Bild 55b) konnte der zuerst beschriebene Anstieg der Dämpfung bei kleinen relativen Durchbiegungen nur noch bei den Balken B2 und B3 beobachtet werden, während in derselben Prüfphase mit der Zusatzmasse $\Delta M_{2}$ (1. Figur in Bild 55c) ein solcher Anstieg überhaupt nicht mehr festgestellt werden konnte. Dies dürfte seine Ursache darin haben, dass bei der Durchführung der Beanspruchungsstufen mit den Zusatzmassen $\Delta M_{1}$ und $\Delta M_{2}$ die Risse schon weitgehend ausgebildet waren und hier somit ähnliche Verhältnisse vorlagen wie bei den Beanspruchungsstufen der 1. Prüfphase ohne Zusatzmassen.

2. Prüfphase (nach Abschluss der Rissbildung):

In der 2. Prüfphase (2. Figur in den Bildern 55a, b und c), nach vollständiger Ausbildung der Risse, wurden bei kleinen relativen Durchbiegungen bzw. Rissweiten sehr hohe Dämpfungswerte festgestellt. Mit zunehmender Durchbiegung nahm die Dämpfung dann aber sehr stark ab und strebte bei grösseren Durchbiegurigen nahezu asymptotisch einem ziemlich tiefen Endwert zu.

3. Prüfphase (nach Abschluss der Rissbildung, verkleinerte Stützweite):

In der 3. Prüfphase (3. Figur in den Bildern 55a, b und c), bei etwa doppelt so hoher Eigenfrequenz der Versuchsbalken, wurde ein ähnliches Dämpfungsverhalten, wie es bei den Beanspruchungsstufen der 2. Prüfphase beschrieben wurde, beobachtet.

\section{Vergleich der Dämpfung von verschiedenen Balken:}

Bei gleich grossen relativen Durchbiegungen wiesen die schwächer armierten Versuchsbalken in allen Prüfphasen eine höhere Dämpfung auf als die stärker armierten Versuchsbalken. 
Bei gleich grossen relativen Durchbiegungen wiesen die Leichtbetonbalken in allen Prüfphasen eine etwas geringere Dämpfung auf als die entsprechenden Betonbalken.

Die Bilder 56a, b und c zeigen dieselben Dämpfungswerte wie die Bilder 55a, $b$ und $c$ in Funktion der relativen Stahlspannung $\sigma_{e, r e l}$ (anstatt der relativen Durchbiegung $\delta_{\text {max, rel }}$ ). Wegen des praktisch linearen Zusammenhangs der Uurchbiegung und der Stahldehnung (vgl. Bild 45) haben die aufgezeichneten Kurven einen ähnlichen Verlauf wie diejenigen der Bilder $55 a$, b und $c$.

In den Bildern 57a, b und $c$ sind die anhand der Beanspruchungsstufen 4.5-..... während eines einzelnen Ausschwingversuches gewonnenen logarithmischen Dekremente $\vartheta$ in Funktion der relativen Durchbiegung $\delta_{\text {rel }}$ aufgetragen. Dabei wurden jedoch nicht nur die ersten zehn Amplituden (Abschnitt I in Bild 33) ausgewertet und aufgetragen wie in den Bildern 55a, b, c, bzw. 56a, b, c, sondern der gemessene Amplitudenverlauf wurde in weitere Abschnitte zu zehn Perioden unterteilt (Abschnitte I, II, III, etc. in Bild 33). Für jeden Abschnitt wurde das logarithmische Dekrement o berechnet und der zu Beginn des zugehöri-

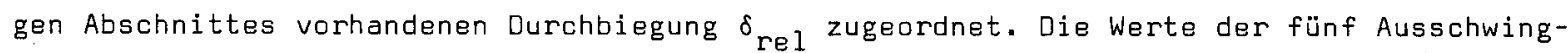
versuche der gewählten Beanspruchungsstufe wurden jeweils gemittelt und in das Diagramm eingetragen. Der in Bild 55 festgehaltene Verlauf der Dämpfung in Abhängigkeit von der Beanspruchungshöhe wurde im wesentlichen bestätigt. Der in Bild 55a beobachtete anfängliche Anstieg der Dämpfung konnte hier allerdings nicht mehr festgestellt werden. Dies ist darauf zurückzuführen, dass die Ausbildung der Risse vor Durchführung der betrachteten Beanspruchungsstufe 4.5-...-1 praktisch abgeschlossen war und nicht mehr dieselben Verhältnisse wie in den ersten Beanspruchungsstufen der 1. Prüfphase vorlagen, wo sich mit zunehmender Beanspruchung noch mehr Risse ausbilden konnten. Die Prüfbalken zeigten hier deshalb ein Dämpfungsverhalten, wie es sich aus der Auswertung der ersten zehn Perioden sämtlicher Ausschwingversuche der 2. Prüfphase ergab (Bild 55).

\subsubsection{Bestimmung aus Resonanzversuchen}

In den Bildern 58a, b und c sind die aus den Resonanzkurven gewonnenen Dämpfungswerte (logarithmisches Dekrement $\vartheta$ ) in Funktion der bei Resonanz erzielten maximalen relativen Durchbiegung $\delta_{\max , r e l}$ aufgetragen. Das logarithmische Dekrement wurde gemäss 61. (20)

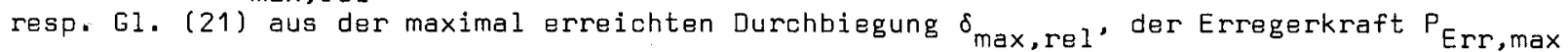
und der gemessenen Steifigkeit kex ermittelt (vgl. Bild 36).

Die Ermittlung der Dämpfung aus der Resonanzkurve war mit grösseren Ungenauigkeiten behaftet als die Bestimmung aus den. Ausschwingversuchen. Erstens bestand vor allem bei kleiner Dämpfung während des Durchfahrens der Resonanzkurve bei jeder noch so sorgfältigen Aenderung der Erregerfrequenz nie absolute Gewissheit, ob das Amplitudenmaximum erreicht wurde. Zweitens mussten auch noch die Erregerkraft und die Steifigkeit bekannt sein, deren Bestimmung ebenfalls mit kleineren Ungenauigkeiten verbunden sein konnte.

Für die 1. und 2. Prüfphase konnte eine befriedigende Uebereinstimmung zwischen den in den Ausschwingversuchen und in den Resonanzversuchen ermittelten Dämpfungswerten gefunden werden. Vor allem bei grösseren relativen Durchbiegungen wurde nahezu dieselbe Dämpfung berechnet. Bei der 3. Prüfphase ist die Uebereinstimmung nur bei grösseren relativen Durchbiegungen befriedigend.

\subsubsection{Wesentliche Erkenntnisse}

Die in den Bildern 55 bis 58 dargestellten Resultate lassen sich wie folgt zusammenfassen :

- In der 1. Prüfphase (fortschreitende Ausbildung der Risse) nahm die Dämpfung - ausgedrückt durch das logarithmische Dekrement $\vartheta$ - bei allen Balken mit fortschreitender. Ausbildung der Risse zuerst zu. Sobald annähernd alle Biegerisse ausgebildet waren, 
nahm jedoch die Dämpfung mit steigerider relativer Durchbiegung resp. Beanspruchung wieder stark ab. Bei hoher Beanspruchung ( $\sigma_{\theta}$,abs nahe bei der fliessgrenze) wurden für die Dämpfung sehr kleine Werte gemessen.

- In der 2. Prüfphase (nach Abschluss der Rissbildung) wurden bei sehr kleinen relativen Durchbiegungen für die Dämpfung sehr hohe Werte gemessen. Mit zunehmender relativer Durchbiegung nahm die Dämpfung ab. Bei grossen relativen Durchbiegungen ergaben sich für die Dämpfung sehr kleine Werte.

- In der 3. Prüfphase (nach Abschluss der Rissbildung, verkleinerte Stützweite) wurde ein zur 2. Prüfphase analoges Verhalten beobachtet. Es wurde kein signifikanter Einfluss der in dieser Phase stark erhöhten Eigenfrequenz auf die Dämpfung festgestellt.

- Mit zunehmendem Armierungsgehalt nimmt die Dämpfung sowohl bei den Betonbalken als auch bei den Leichtbetonbalken ab.

Alle Leichtbetonbalken wiesen im ganzen Beanspruchungsbereich ein etwas geringeres Dämpfungsvermögen auf als die entsprechenden Betonbalken. 
Im Rahmen des Forschungsprojektes "Dynamisches Verhalten von Leichtbeton- und Betonkonstruktionen" wurden am Institut für Baustatik und Konstruktion der Eidgenössischen Technischen Hochschule Zürich Versuche an schlaff armierten Leichtbeton- und Betonbalken durchgeführt. Die vier Leichtbetonbalken und die drei Betonbalken unterschieden sich nebst der verwendeten Betonart in der Grösse der Längsarmierung. Sie wiesen eine Länge von $8.40 \mathrm{~m}$ und einen Rechteckquerschnitt von $40 \mathrm{~cm}$ Breite und $24 \mathrm{~cm}$ Höhe auf. An den als einfache Balken gelagerten Versuchskörpern erfolgten Ausschwing- und Resonanzversuche. Zur Erzeugung der Resonanzschwingungen wurde ein neuartiger ölhydraulischer Schwingungserreger entwickelt.

Die Versuche hatten zum Ziel, den Einfluss der Rissbildung, des Armierungsgehaltes und der Beanspruchungshähe auf die dynamischen Kenngrässen Steifigkeit resp. Eigenfrequenz sowie Dämpfungsvermögen zu untersuchen. Ferner sollte ein Vergleich zwischen Betonbalken und analog ausgebildeten Leichtbetonbalken angestellt werden.

Das Prüfprogramm war für alle Balken analog aufgebaut:

- In einer 1. Prüfphase (Stützweite $1=7.88 \mathrm{~m}$ ) wurde die Stahlspannung, beginnend beim noch praktisch ungerissenen Zustand, sukzessive in Stufen von $0.5 \mathrm{t} / \mathrm{cm}^{2}$ bis nahe an die Fliessgrenze gesteigert. Innerhalb einer durch eine bestimmte maximale Stahlspannung definierten Stufe wurden einerseits Beanspruchungsstufen ohne Zusatzmassen und andererseits Beanspruchungsstufen mit zusätzlichen auf den Balken aufgebrachten Bleimassen angeordnet. Innerhalb jeder Beanspruchungsstufe wurden mehrere Ausschwingversuche durchgeführt und je eine Resonanzkurve mit steigender und fallender Erregerfrequenz aufgenommen.

- In der 2. Prüfphase (Stützweite $1=7.88 \mathrm{~m}$ ) wurde dasselbe Prüfprogramm wiederholt. Damit konnte der Einfluss der Belastungsgeschichte resp. der Ausbildung der Risse - die Risse waren während der 1. Prüfphase entstanden - genauer erfasst werden.

- In der 3. Prüfphase (Stützweite $1=4.84 \mathrm{~m}$ ind zwei Kragarme mit einer Länge von je $1.78 \mathrm{~m}$ ) wurde dasselbe Prüfprogramm wie bei der 1. und 2. Prüfphase durchgeführt.

Bei sämtlichen Balken wurden in drei verschiedenen Querschnitten die Durchbiegungen, die Stah 1 - und Betondehnungen sowie die Balkenbeschleunigungen aufgenommen. Zudem konnte am Schwingungserreger die Erregerkraft ermittelt werden.

Die Ergebnisse lassen sich wie folgt zusammenfassen:

\section{Steifigkeiten}

Die durch die Rissbildung bewirkte Verminderung der Steifigkeit war bei den schwach armierten Versuchsbalken erwartungsgemäss grösser als bei den stark armierten Bałken.

Bei den Leichtbetonbalken betrug die maximale Verminderung der Steifigkeit gegenüber dem ungerissenen Zustand 57 bis $71 \%$ und bei den Betonbalken 68 bis $64 \%$.

An den verschiedenen Versuchsbalken konnte, je nach Art und Zeitpunkt der durchgeführten Beanspruchungsstufe, sowohl unterlineares Verhalten (nach links kippende Resonanzkurve) als auch lineares Verhalten (vertikale Resonanzkurve) und überlineares Verhalten (nach rechts kippende Resonanzkurve) beobachtet werden. 


\section{Beschleunigungen}

Die stärker armierten Versuchsbalken wiesen bei gleich grosser relativer Durchbiegung stets eine grössere Beschleunigung auf als die schwach armierten Balken.

Die Leichtbetonbalken wiesen bei gleich grosser relativer Durchbiegung stets eine grössere Beschleunigung auf als die entsprechenden Betonbalken.

\section{Biegemomente und Querkräfte}

Die Biegemomente und Querkräfte infolge der dynamischen Belastung waren bei sich entsprechenden Leichtbeton- und Betonbalken stets annähernd gleich gross.

\section{Dehnungen}

Die relativen Stahldehnungen von sich entsprechenden Leichtbeton- und Betanbalken waren bei gleicher relativer Durchbiegung stets ungefähr gleich gross.

Die relativen Betondehnungen von sich entsprechenden Leichtbeton- und Betonbalken lagen bei gleicher relativer Durchbiegung stets in derselben Grössenordnung.

\section{Rissverhalten}

Die relative Rissöffnung lag bei allen Versuchsbalken in derselben Grössenordnung und überstieg nie den Wert von $25 / 100 \mathrm{~mm}$.

\section{Eigenfrequenzen}

Mit wachsender Beanspruchung nahm die Eigenfrequenz infolge der zunehmenden Rissbildung stark ab.

Die Abnahme der Eigenfrequenz gegenüber dem ungerissenen Zustand war bei den schwach armierten Balken grösser als bei den stärker armierten Balken.

Die Abnahme der Eigenfrequenz gegenüber dem ungerissenen Zustand betrug bei den Leichtbetonbalken 33 bis $44 \%$, bei den Betonbalken 47 bis $54 \%$.

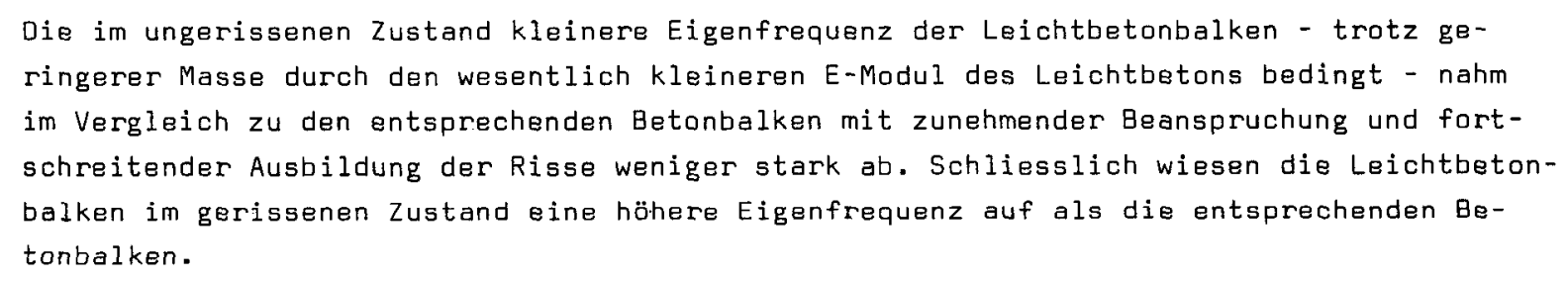

\section{Dämpfung}

In Bild 59 ist der Verlauf der Dämpfung - ausgedrückt durch das logarithmische Dakrement $\checkmark$ - in Funktion der maximalen relativen Durchbiegung in Balkenmitte schematisch dargestellt. Die folgende Numerierung bezieht sich auf die Numerierung in Bild 59.

1. Bei allen Balken nahm die Dämpfung mit zunehmender Durchbiegung resp. fortschreitender Ausbildung der Risse (1. Prüfphase, Stützweite $1=7.88 \mathrm{~m}$ ) zuerst zu.

2. Während der Rissbildung wurde ein Zustand erreicht, bei welchem die Dämpfung nicht mehr weiter anstieg.

3. Sobald annähernd alle Biegerisse ausgebildet waren (gegen das Ende der 1. Prüfphase), nahm die Dämpfung mit steigender Durchbiegung wieder ab. Bei grossen Durchbiegungen (entsprechend einer Stahlspannung nahe bei der Fliessgrenze) wurden für die Dämpfung 
sehr kleine Werte gemessen.

4. Bei der nochmaligen Durchführung des gesamten Prüfprogrammes an denselben Versuchskörpern (2. Prüfphase, Stützweite $1=7.88 \mathrm{~m}$ ) ergab sich bei allen Balken bei kleinen relativen Durchbiegungen eine im Vergleich zu den bei Versuchsbeginn (1. Prüfphase) gemessenen Werten sehr hohe Dämpfung. Mit zunehmender Durchbiegung nahm dann die Dämpfung wieder ab.

5. Bei grossen Durchbiegungen wurden für die Dämpfung sehr kleine Werte gemessen.

6. Durch Verkleinern der Stützweite (3. Prüfphase, Stützweite $1=4.84 \mathrm{~m}$ ) - die Eigenfrequenz wurde dadurch etwa verdoppelt - wurde ein zur 2. Prüfphase analoges Verhalten beobachtet. Auch hier nahm die Dämpfung mit zunehmender Durchbiegung erheblich ab.

Es wurde kein signifikanter Einfluss der Eigenfrequenz auf die Dämpfung festgestellt.

7. Mit zunehmendem Armierungsgehalt nahm die Dämpfung ab.

8. Die Leichtbetonbalken wiesen im ganzen Beanspruchungsbereich ein etwas geringeres

Dämpfungsvermägen als die entsprechenden Betonbalken auf.

Die interessanten Ergebnisse dieser Versuche können mit den heutigen Kenntnissen nicht restlos erklärt werden. Es besteht deshalb die Absicht, in einer theoretischen Arbeit die aufgezeigten Phänomene anhand von möglichst wirklichkeitsnahen Modellen noch weiter zu untersuchen. 
Dans le cadre du projet de recherche "Comportement dynamique des ouvrages en béton léger et en béton conventionnel", on a effectué, à l'Institut de statique et de constructions de l'Ecole Polytechnique Fédérale de Zurich (EPFZ), des essais dynamiques sur sept poutres en béton léger (quatre) et en béton conventionnel (trois). Ces poutres simples mesuraient $8.40 \mathrm{~m}$ en longueur et avaient une section rectangulaire de $40 \mathrm{~cm}$ en largeur et de $24 \mathrm{~cm}$ en hauteur. Le pourcentage géométrique d'armature longitudinale fut choisi comme variable principale. Un nouveau générateur de vibrations hydraulique fut utilisé pour produire les sollicitations dynamiques (vibrations transversales, résonance).

Les essais avaient comme but principal, l'étude de l'influence des fissures, du pourcentage gémétrique d'armature et du niveau des sollicitations sur les valeurs caractéristiques dynamiques des poutres soit la rigidité, la fréquence propre et le coefficient d'amortissement. De plus, les essais ont permis de comparer le comportement dynamique des poutres en béton léger avec celui de poutres analoques en béton conventionnel.

Le programme des essais, valable pour les sept poutres, comprenait trois phases:

- Durant la première phase (portée $1=7.88 \mathrm{~m}$ ), l'accroissement des tensions dans l'acier, à partir du stade pratiquement non-fissuré, s'élevait à $0.5 \mathrm{t} / \mathrm{cm}^{2}$ jusqu'au voisinage de la limite d'écoulement. Durant une étape, définie par une tension maximale dans les armatures, on a testé les poutres à l'aide de masses de plomb. Durant chaque étape de charge, et pour toutes les poutres avec et sans masses de plomb, on a effectue plusieurs essais de vibrations et déterminé une courbe de résonance avec fréquence perturbatrice ascendante et une avec fréquence perturbatrice descendante.

- Durant la deuxième phase (portée $1=7.88 \mathrm{~m}$ ) on a répété le programme de la première phase dans le but d'étudier l'influence de l'histoire du chargement et de la fissuration sur le comportement dynamique des poutres.

- Durant la troisième phase (portée $1=4.84 \mathrm{~m}$ avec deux poutres cantilever de $1.78 \mathrm{~m}$ de portées, on a exécuté le même programme que dans la deuxième phase.

Pour toutes les poutres, on a mesuré dans trois sections choisies, la flèche, les tensions dans le béton et dans les armatures ainsi que les accélérations. De plus, la force perturbatrice du générateur de vibrations put être détẹminée.

Les résultats des essais peuvent être résumés comme suit:

\section{Rigidités}

La diminution de rigidité par rapport à celle du stade non-fissuré était plus forte pour les poutres faiblement armées que pour celles fortement armées et ceci en accord avec les prévisions.

Pour les poutres en béton léger, la plus grande diminution de rigidité par rapport à celle du stade non-fissuré se situait entre 57 et $71 \%$ tandis qu'elle variait entre 68 et $84 \%$ pour les poutres en béton conventionnel.

Pour les différentes poutres on avait pu observer, suivant le genre et le moment de l'étape de charge, des courbes de résonance basculant vers la gauche (comportement souslinéaire), des courbes de résonance verticales (comportement linéaire) et des courbes de résonance basculant vers la droite (comportement sur-linéaire). 


\section{Accélérations}

Pour les poutres plus fortement armées, on a observé, systématiquement, une plus grande accélération que pour les poutres correspondantes en beton conventionnel.

Les poutres en béton léger avaient, pour une même flèche, systématiquement une plus grande accélération que les poutres correspondantes en béton conventionnel.

\section{Moments de flexions et efforts tranchants}

Les moments de flexion et les efforts tranchants causés par les sollicitations dynamiques avaient le même ordre de grandeur aussi bien pour les poutres en béton conventionnel que pour celles en béton léger.

\section{Deformations}

Les allongements relatifs de l'acier des poutres en béton léger etaitent, pour les mêmes flèches relatives, environ analogues à celles des poutres en béton conventionnel. Les déformations relatives comparées des poutres en béton léger avec poutres en béton conventionnel étaient, pour les mêmes flèches relatives, du même ordre de grandeur.

\section{Comportement des fissures}

L'ouverture relative des fissures etait du même ordre de grandeur pour tous les essais et ne dépassait jamais la valeur de $25 / 100 \mathrm{~mm}$.

\section{Fréquences propres}

Plus les sollicitations croissaient plus la fréquence propre décroissait, cela etant dû à l'augmentation de la fissuration.

La diminution de la fréquence propre de l'état homogène à l'état fissuré a été plus importante pour les poutres légèrement armées que pour les poutres plus fortement armés. La diminution de la fréquence propre de l'état homogène à l'état fissuré fut de 33 à $44 \%$ pour les poutres en béton léger, de 47 à $54 \%$ pour les poutres en béton conventionnel.

La fréquence propre des poutres en béton léger à l'état homogène subit une diminution moins grande lors de l'augmentation de la charge dynamique et de la fissuration malgré une masse plus faible, le module d'élasticité du béton léger est beaucoup plus petit - que celle des poutres en béton conventionnel. Enfin, les poutres en béton léger eurent une fréquence propre plus élevée que les poutres correspondantes en béton canventionnel.

\section{Amortissement}

Sur le diagramme 59 on peut voir schematiquement l'amortissement - exprimé par le décrement logarithmique $v$ - en fonction de la flèche relative maximale. La numérotation suivante correspond à la numérotation du diagramme 59 .

1. L'amortissement augmentait pour toutes les poutres avec la croissance des fissures resp. croissance de la flèche (première phase, portée $1=7.88 \mathrm{~m}$ ).

2. Pendant la formation des fissures on avait atteint un point où l'amortissement n'augmentait plus pendant la croissance de la flèche.

3. Aussitôt que toutes les fissures de flexion s'étaient développés, l'amortissement diminuait avec la croissance de la flèche (vers la fin de la première phase). Pour de grandes flèches (correspondantes à une tension dans l'acier, au voisinage de la limite d'écoulement) de très petites valeurs d'amortissement ont été mesurés.

4. Dans une deuxième phase (portée $1=7.88 \mathrm{~m}$ ), l'essais a été répété dans tout son dé- 
roulement aux mêmes poutres. Pour de faibles flèches l'amortissement fut très élevé comparé aux valeurs obtenues en début d'essais, l'amortissement diminuant lors de l'augmentation de la flèche.

5. De très petites valeurs d'amortissement ont été mesurées pour de très grandes flèches.

6. Après avoir raccourci la portée (troisième phase, portée $1=4.84 \mathrm{~m}$ ) - la fréquence propre est environ dedoublée - un comportement analogue à la deuxième phase a été observé, l'amortissement diminuant ici aussi avec l'augmentation de la flèche.

7. Plus le pourcentage d'armature est grand, plus l'amortissement est petit.

8. Les poutres en béton léger montrèrent dans tout le domaine de sollicitation un amortissement légèrement moindre que les poutres correspondantes en béton conventionnel.

Ces résultats très intéressants ne peuvent être parfaitement expliqués avec les connaissances existant actuellement; c'est pourquoi l'intention est d'expliquer les phénomènes observés avec des modèles et de présenter ces explications dans un travail ultérieur. 
Within the framework of the research project: "Dynamic behaviour of lightweight and other concrete structures" tests were carried out at the Institute of Structural Engineering at the Swiss Federal Institute of Technology Zurich on normally reinforced lightweight and normal concrete beams. Four lightweight and three normal concrete beams were used. These differed not only in the type of concrete used but also in the amount of axial reinforcement. The beam dimensions were as follows: length $8.40 \mathrm{~m}$, rectangular section $40 \mathrm{~cm}$ width $\times 24 \mathrm{~cm}$ height. Under simple support conditions both free vibration and resonance tests were carried out. A new type of hydraulic vibrator was developed for the resonance tests.

The purpose of the tests was to investigate the influence of crack development, amount of reinforcement and intensity of loading on the dynamic stiffness (i.e. change of eigenfrequencies) and damping characteristics. Further, a comparison should be made between the behaviour of lightweight and normal concrete beams.

The testing program was the same for all the beams:

- In the first phase (span $l=7.88 \mathrm{~m}$ ) the steel stress was progressively increased in steps of $0.5 \mathrm{t} / \mathrm{cm}^{2}$ from the practically uncracked state up to a stress near to the yield stress. Within a load step corresponding to a particular steel stress, the beam was tested with and without added masses. Lead was used to increase the mass. Several free vibration tests were carried out within each loading step and one resonance test, sweeping the excitation frequency both up and down.

- In the second phase (span $1=7.88 \mathrm{~m}$ ) the program was repeated. Thereby, the influence of the load history and of the cracks developed during the first phase of testing could be examined in detail.

- In the third phase (span $1=4.84 \mathrm{~m}$, with two cantilevers of length $1.78 \mathrm{~m}$ each) the same procedure was followed as in the previous test phases.

For all beams accelerations were measured in three different sections, together with the bending deflections and the strains in the steel and the concrete. In addition, the excitation force was determined.

The re'sults of the investigation may be summarized as follows:

\section{Stiffness}

The drop in stiffness compared to the uncracked state was as expected greater for the beams with low reinforcement than for those which were heavily reinforced.

For the lightweight concrete beams the maximum reduction in stiffness was between 57 and $71 \%$ compared to the normal concrete beams with a 68 to $84 \%$ reduction.

Tests on the various beams showed that, according to the nature and time at which they were carried out, it was possible to have non-linear behaviour (with the resonance curve bending to the left), also linear behaviour (vertical resonance curve) and non-linear behaviour (with the resonance curve bending to the right).

\section{Acceleration}

The heavily reinforced beams always exhibited, for the same relative deflexion, a higher acceleration than was the case with the weakly reinforced beams. 
The lightweight concrete beams always exhibited, for the same relative deflexion a higher acceleration than the corresponding normal concrete beams.

\section{Bending Moment and Shear Force}

The corresponding values of bending moments and shear forces due to dynamic loading were, for corresponding lightweight and normal concrete beams, always close together.

\section{Strains}

The relative strains in the steel for corresponding lightweight and normal concrete beams were, for the same relative deflexion, always practically equal.

The relative strains in the concrete for corresponding lightweight and normal concrete beams were, for the same relative deflexion, always of the same order of magnitude.

\section{Cracking}

The relative openings of the cracks were always of the same order of magnitude for all the beams tested and never exceeded the value $25 / 100 \mathrm{~mm}$.

\section{Eigenfrequencies}

With increasing loading, the eigenfrequencies reduced considerably due to the progressive development of cracks. The reduction in the eigenfrequencies compared to those of the uncracked state was, for the case of the weakly reinforced beams greater than for the heavily reinforced beams. For the lightweight concrete beams the drop in eigenfrequency amounted to 33 to $44 \%$, while for the normal concrete beams it was 47 to $54 \%$.

\footnotetext{
The smaller eigenfrequency of the lightweight concrete beams in the uncracked state despite the smaller mass the elastic modulus is much less - fell, in comparison to the normal concrete beams, less with increasing loading and crack development.

It may be concluded that for corresponding lightweight concrete and normal concrete beams the lightweight concrete beams exhibited a higher eigenfrequency in the cracked state.
}

\section{Damping}

The variation of the damping, expressed in terms of the logarithmic decrement $v$, as a function of the maximum relative deflexion at the midspan of the beam is shown schematically in Fig. 59. The numbering scheme in the following corresponds to that of Fig. 59 .

1. In all the beams for increasing deflexion and progressive crack development (1. phase with span $l=7.88 \mathrm{~m}$ ), the damping increased at first.

2. During the cracking state a condition was reached, at which the damping did not increase any longer.

3. When nearly all the bending cracks had been formed (towards the end of the 1. phase) the damping began to decrease with increasing deflexion. At high deflexions (corresponding to a steel stress near to the yield stress limit) very small damping values were obtained.

4. When the complete test procedure was repeated on the same test specimen (2. phase, span $1=7.88 \mathrm{~m}$ ) there resulted for small relative deflexions, for all tests, damping values which were very high in comparison to the values previously obtained. With increasing deflexion the damping decreased. 
5. Again at very high deflexions very small values of damping were measured.

6. By decreasing the span (3. phase, span $1=4.84 \mathrm{~m}$ ), which corresponds to about a doubling of the eigenfrequency, a behaviour analogous to that observed in the second phase was obtained. Again the damping decreased with increasing deflexion.

7. Damping decreased with increasing amounts of reinforcement. 6. The lightweight concrete beams exhibited, for the whole range of loading, a some-
what smaller damping capacity than the corresponding normal concrete beams.

The interesting results of these tests cannot be explained with complete satisfaction according to our present state of knowledge. We intend, therefore, to investigate the phenomena described herein more thoroughly with the aid of mechanical models and present the work in a subsequent report. 
VERDANKUNGEN

Der varliegende Versuchsbericht ist im Rahmen des ersten Teils des Forschungsprojektes "Dynamisches Verhalten von Beton- und Leichtbetonkonstruktionen" ausgearbeitet worden. Dieses Projekt wurde durch einen finanziellen Beitrag der Firma

Hunziker \& Cie AG, Baustoffabriken, Zürich

ermöglicht. Für die grosszügige Unterstützung möchten die Verfasser der Firma Hunziker und insbesondere ihrem Präsidenten, Herrn dipl. Ing. W. Thut, herzlich danken.

Für die Veberlassung der benötigten Armierungsstähle danken die Verfasser der Firma von Moos Stahl AG, Luzern.

Der notwendige Vorversuch konnte an der Eidgenössischen Materialprüfungs- und Versuchsanstalt (EMPA) in Dübendorf.durchgeführt werden. Hierfür sei Herrn Prof. Dr. Th. Erismann, Direktionspräsident, und Herrn Dr. M. Ladner, Vorsteher der Abteilung für Massivbau, der beste Dank ausgesprochen.

Herr dipl. Ing. M. Baumann betreute die messtechnischen Belange und Herr K. Bucher half bei der Entwicklung der Versuchsanlage mit. Herr L. Sieger zeichnete die Bilder und Frl. S. Burki schrieb die Druckbogen. Herr G. Göseli betreute die Drucklegung. Für die wertvolle Unterstützung und die gute Zusammenarbeit danken die Verfasser allen Genannten wie auch allen anderen, die zum Entstehen dieses Berichtes beigetragen haben, bestens. 
BEZE ICHNUNGEN

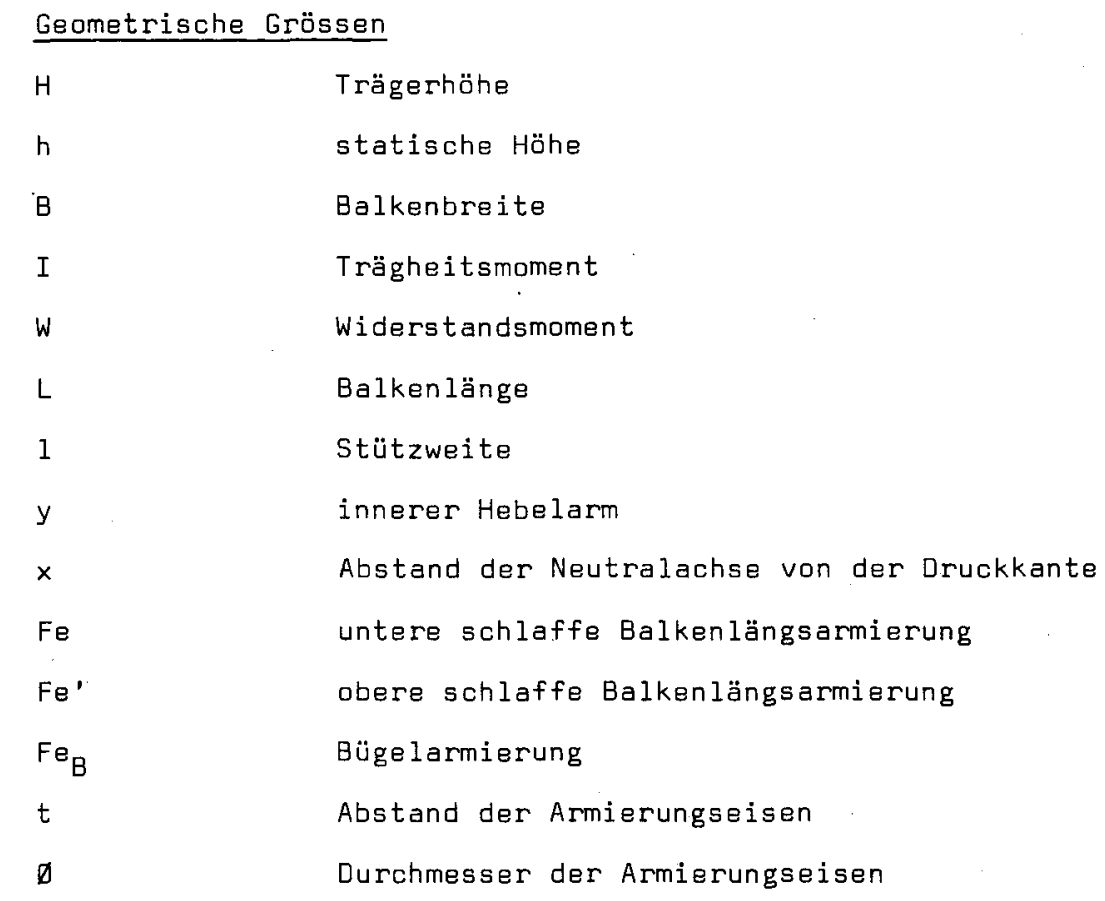

\section{$\mathrm{H}$}

h

I

w

L

1

$y$

$x$

$\mathrm{Fe}$

$\mathrm{Fe}{ }^{\prime}$

$\mathrm{Fe}_{\mathrm{B}}$

$t$

$\emptyset$

Trägerhöhe

statische Höhe

Balkenbreite

Trägheitsmoment

Widerstandsmoment

Balkenlänge

Stützweite

innerer Hebelarm

Abstand der Neutralachse von der Druckkante

untere schlaffe Balkenlängsarmierung

obere schlaffe Balkenlängsarmierung

Bügelarmierung

Abstand der Armierungseisen

Durchmesser der Armierungseisen

B

\section{$\underline{\text { Kraftgrössen }}$}

$P_{A}$
$P_{E r r}$
$P_{\text {statisch' }} P_{\text {st }}$
$Z$
$R$
$F_{D}$
$F_{C}$
$M$
$M_{P I}$
$M_{R}$.
$Q$

\section{Massen}

$m$

$\bar{m}$

G

$\Delta M$

$\Delta M_{1}$

$\Delta M_{2}$

\section{Festigkeitswerte, Spannungen}

$B_{w}$

${ }^{B} p$

$\beta_{b z}$

Biegemoment

Rissmoment

Querkraft

Zusatzmasse
Kraft beim Ausschwingversuch

Erregerkraft beim Resonanzversuch

Einzellast beim statischen Last-Durchbiegungs-Diagramm

Zugkraft der Längsarmierung

bewegungshemmende Kraft des Einmassenschwingers

Dämpfungskraft

Dämpfungskraft bei Coulomb-Reibung

Plastisches Moment

verteilte Masse des Balkens

bewegliche Masse des Schwingungserregers

gesamte Masse des Schwingungserregers

halbe Zusatzmasse $\sim 152.4 \mathrm{~kg} / \mathrm{m}^{\prime}$

ganze Zusatzmasse $\sim 295.2 \mathrm{~kg} / \mathrm{m}^{\prime}$

W

Würfeldruckfestigkeit des Betons, resp. Leichtbetons

Zylinderdruckfestigkeit des Betons, resp. Leichtbetons

-Biegezugfestigkeit des Betons, resp. Leichtbetons 


$\sigma_{e}$
$\sigma_{e, 2.0}$
$\beta_{Z}$
$E_{e}$
$E_{B}$
$E_{L B}$
$E_{5-50}$
$E_{5-100}$
$E_{5-150}$
$E_{5-200}$
$\tau$

\section{Verformungen}

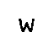

z

V

k

kunger

a

$\delta$

$\delta_{\max , \text { re } 1}$

$\delta_{\max , \text { rel }}$

$\bar{\delta}$

$\varepsilon_{\mathrm{e}}$

$\varepsilon_{E, m}$

$\varepsilon_{\mathrm{e}, \max }$

$\varepsilon_{\text {e,rel }}$

$\varepsilon_{\text {e,rel }}$

$\varepsilon_{f}$

$\lambda_{\mathrm{GI}}$

$\lambda_{5}, \lambda_{10}$

$\varepsilon_{b}$

$\varepsilon_{\text {b, rel }}$

$\varepsilon_{b, r e l}$

$\varepsilon_{u}$

r kex

Stahlspannung

Streckgrenze des Stahls

Zugfestigkeit des Stahls

Elastizitätsmodul des Stahls

Elastizitätsmodul des Betons

Elastizitätsmodul des Leichtbetons

Sekantenmodul bei Belastung von 5 bis $50 \mathrm{~kg} / \mathrm{cm}^{2}$

Sekantenmodul bei Belastung von 5 bis $100 \mathrm{~kg} / \mathrm{cm}^{2}$

Sekantenmodul bei Belastung von 5 bis $150 \mathrm{~kg} / \mathrm{cm}^{2}$

Sekantenmodul bei Belastung von 5 bis $200 \mathrm{~kg} / \mathrm{cm}^{2}$

Schubspannung

absolute Verschiebung des Einmassenschwingers

relative Verschiebung des Einmassenschwingers

Vergrösserungsfaktor

Steifigkeit des Einmassenschwingers

gemessene Balkensteifigkeit

Steifigkeit des ungerissenen Balkens

Kolbentiub

Durchbiegung

maximale relative Durchbiegung beim Resonanzversuch

relative Anfangsdurchbiegung infolge von $P_{A}$ beim Ausschwingversuch

Durchbiegung im Viertelspunkt, resp. Kragarmende

Stah Idehnung

mittlere Stahldehnung

maximale Stahldehnung im Riss

maximale relative Stahldehnung beim Resonanzversuch

relative Anfangsstahldehnung beim Ausschwingversuch

Fliessdehnung des Stahls

Gleichmassdehnung des Stahls

Bruchdehnung des Stahls, über 5 D, resp. 10 Ø̈̈ber Einschnürungszone gemessen

Betondehnung

maximaie relative Betondehnung beim Resonanzversuch

relative Anfangsbetondehnung beim Ausschwingversuch

Betonbruchstauchung

Rissäffnung

\section{Dynamische Grössen}

$\Omega$

Erregerfrequenz

$\omega$

Eigenkreisfrequenz

Eigenfrequenz 
$\dot{E}$

Dehngeschwindigkeit

\section{Dimensionslose Parameter}

$\mu$,

$\Psi$

K

n

\section{Indices}

b

e

th

ex

f

\section{g}

B

L官

id

unger

ger

$d$

statisch

stationär

$+$

$-$

0

rel

abs

Armierungsgehalt (untere Längsarmierung)

Armierungsgehalt (obere Längsarmierung)

Brucheinschnürung

Verbundkoeffizient

Verhältnis $E_{e} / E_{B}$

Beton, Leichtbeton

Stahl

theoretisch

experimentell

Fliessen

ständige Last

Beton

Leichtbeton

ideell

ungerissen

gerissen

dynamisch

statisch

stationär

Schwingung nach unten (hochgestellter Index)

Schwingung nach oben (hochgestellter Index)

in Ruhelage gemessen (tiefgestellter Index)

relativ, im Ausschwing- resp. Resonanzversuch gemessene Werte ohne die in der Ruhelage gemessenen Werte (tiefgestellter. Index)

absolut, im Ausschwing- resp. Resonanzversuch gemessene Werte inkl. den in der Ruhelage gemessenen Werten (tiefgesteliter Index) 
[1] W.C. Hurty, M.F. Rubinstein: "Dynamics of Structures", Prentice Hall, New Jersey, 1964 .

[2] R.W. Clough, J. Prenzien: "Dynamics of Structures", Mc Graw Hill, 1975.

[3] N.M. Newmark, E. Rosenblueth: "Fundamentals of Earthquake Engineering, Prentice Hali, New Jersey, 1971.

[4] D. Pfaffinger: "Anwendung der Finiten Elemente in der Dynamik", Vorlesung an der ETH Zürich, 1975.

[5] K.Th. Teichen: "Ueber die innere Dämpfung von Beton", Otto-Graf-Institut, Stuttgart, 1968.

[6] J. Ali, C.E. Kesler: "Rheology of Concrete: A Review of Research", University of Illinois, Bulletin $V 62$, No. 68, March 1965 .

[7] M. Wakayabashi: "Studies on Damping and Energy Absorption of Structures", IVBHSymposium, Einführungsbericht, Lissabon, 1973.

[8] E. Bock: "Ueber den dynamischen E-Modul und die Dämpfung von Betonbalken verschiedener Bewehrung bei Biegungs- und Längsschwingungen, 1939, Dissertation, Berlin.

[9] G. Ehlers: "Das Elastizitätsmass des Betons bei Schwingungen", Beton und Eisen, 40. Jahrgang, Heft 20, S. 268-272, 1941, Heft 21, S. 280-282, 1941.

[10] H. Lenk: "Ueber das Schwingungsverhalten von Spannbeton- und Stahlbetonbalken", Dissertation, Stuttgart, 1963.

[11] S. Penzien: "Damping Characteristics of Prestressed Concrete", ACI Journal Proc., Vol. 61. No. 9 (Sept. pp 1125-1148), 1964.

[12] G. Denkhaus, G. Dück: "Ueber das Verhalten von Spannbetonbalken bei Biegeschwingungen", Beton- und Stahlbetonbau, Heft 3, 1952.

[13] F.P. Müller: "Die Ermittlung der Abweichung vom linearen Formänderungsgesetz sowie die Dämpfung von Spannbeton durch Ausschwingversuche", Beton- und Stahlbetonbau, 54. Jahrgang, Heft 11, S. 276-278.

[14] G.D. Fritz-de-la-Orta: "Ueber das Verhąten von Spannbeton-Bauteilen bei dynamischen Beanspruchungen, insbesondere bei Erdbeben", München 1958.

[15] St. Soretz: "Ermüdungseinfluss im Stahlbeton", Betonstahl in Entwicklung, Heft 24 , 1965.

[16] St. Soretz: "Beitrag zur Ermüdungsfestigkeit von Stahlbeton", Betonstahl in Entwicklung, Heft 57, 1974.

[17] M. Baumann, R. Dieterle, H. Bachmann: "Neuartige Belastungs- und Messeinrichtungen für Schwingversuche an armierten und vorgespannten Betonprüfkörpern". Institut für Baustatik und Konstruktion, ETH Zürich, Bericht Nr. 88, 1978. (Sonderdruck aus der Schweizerischen Bauzeitung, 96. Jahrgang, Heft 11, 5. 276-278.) 


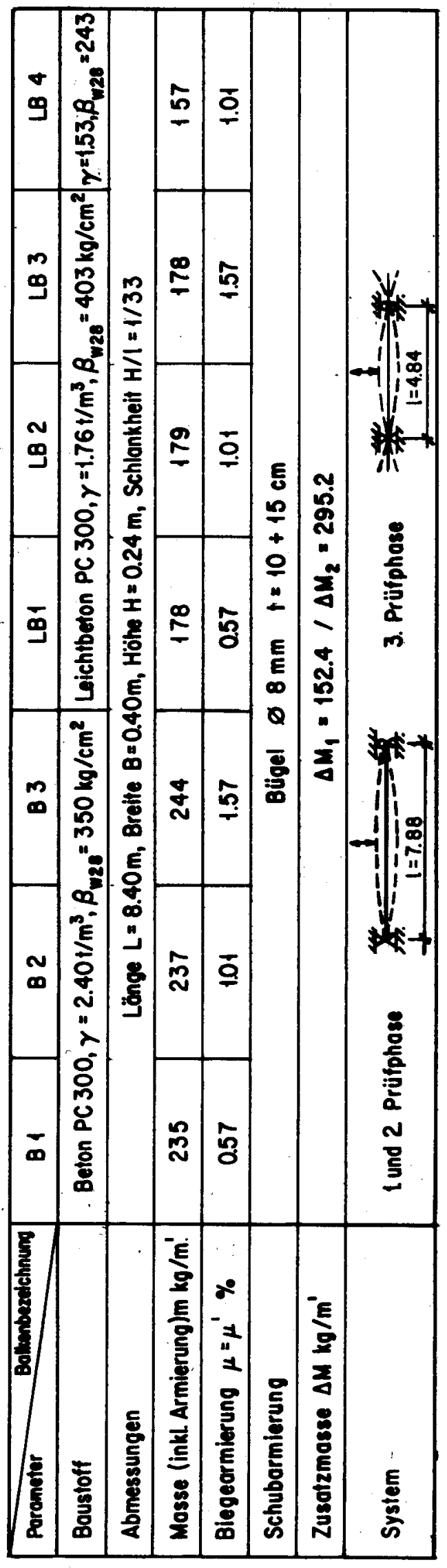

\begin{tabular}{|c|c|c|c|c|c|}
\hline 㝘哭 & \multicolumn{4}{|c|}{ 总 } & \\
\hline$\rightarrow \infty$ & $\overline{\tilde{v}} \overline{0}$ & 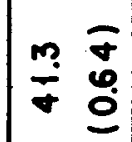 & $\left(\begin{array}{ll}n & 0 \\
\infty & 0 \\
m & 0\end{array}\right.$ & 家 & \\
\hline$\stackrel{0}{2}$ & $\underset{\sim}{\stackrel{m}{o}}$ & $\mid \begin{array}{ll}1 & \overline{8} \\
0 & 0 \\
0 & 0\end{array}$ & 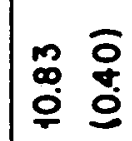 & 웅 & \\
\hline$\infty$ & 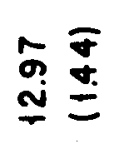 & $\mid \begin{array}{ll}\hat{0} & \overline{0} \\
0 & 0 \\
0 & 0\end{array}$ & 足 & 용 & \\
\hline 2 & 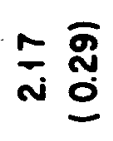 & 幽 & 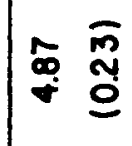 & i⿱ & \\
\hline س & 愈 & 㢳 & : & 包 & \\
\hline$\alpha^{N} \stackrel{N}{\underline{E}}$ & $\stackrel{0}{0}_{0}^{\circ \bar{\delta}}$ & $\frac{0}{6}$ & 훙 & 웅 & \\
\hline & 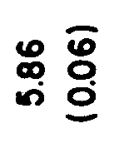 & 突 & 둥 & 范 & \\
\hline w & $\stackrel{\infty}{0}$ & $\ddot{0}$ & $\mathscr{0}$ & م & \\
\hline$\underline{E}=\tilde{E}$ & 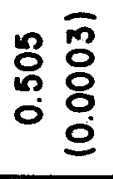 & 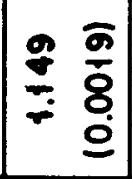 & 옹 & 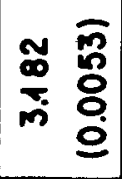 & 产 \\
\hline 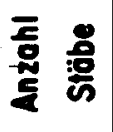 & is & $m$ & $m$ & m & \\
\hline 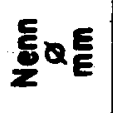 & $\infty$ & $\simeq$ & $\stackrel{0}{-}$ & O & 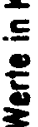 \\
\hline
\end{tabular}

$\stackrel{\because}{\circ}$ 


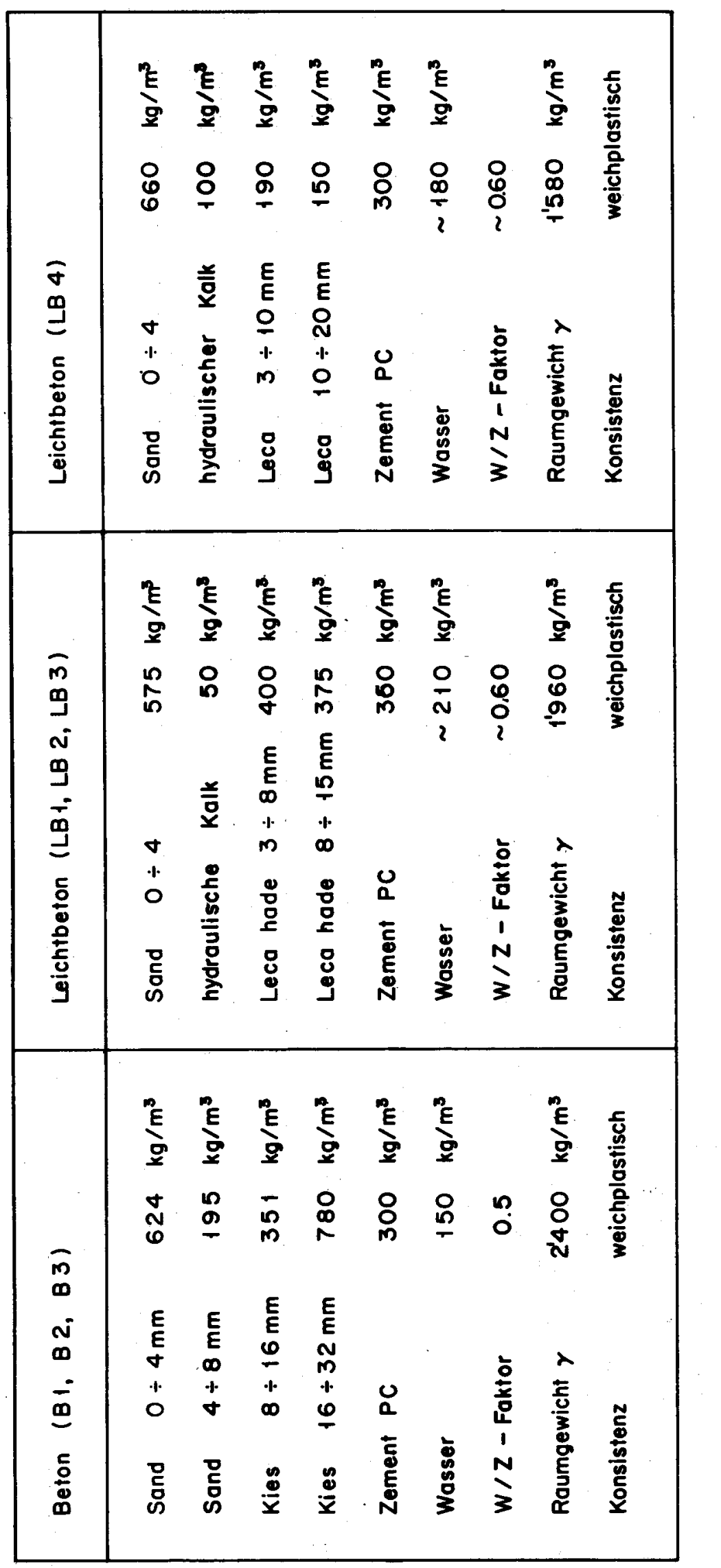

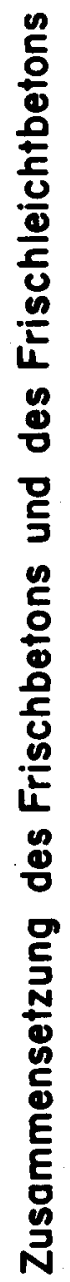

$\ddot{m}$
$\frac{0}{0}$
$\dot{0}$ 


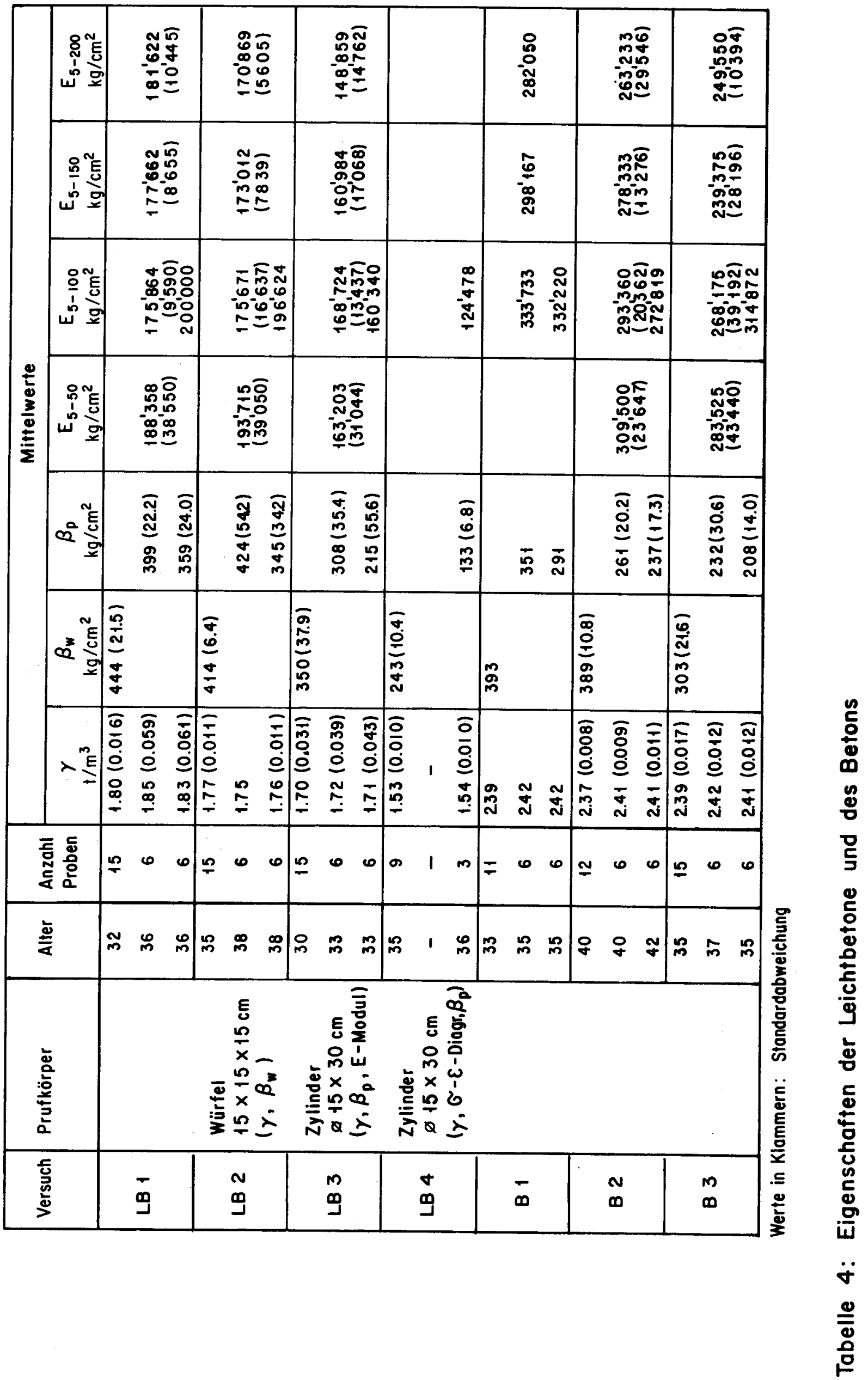




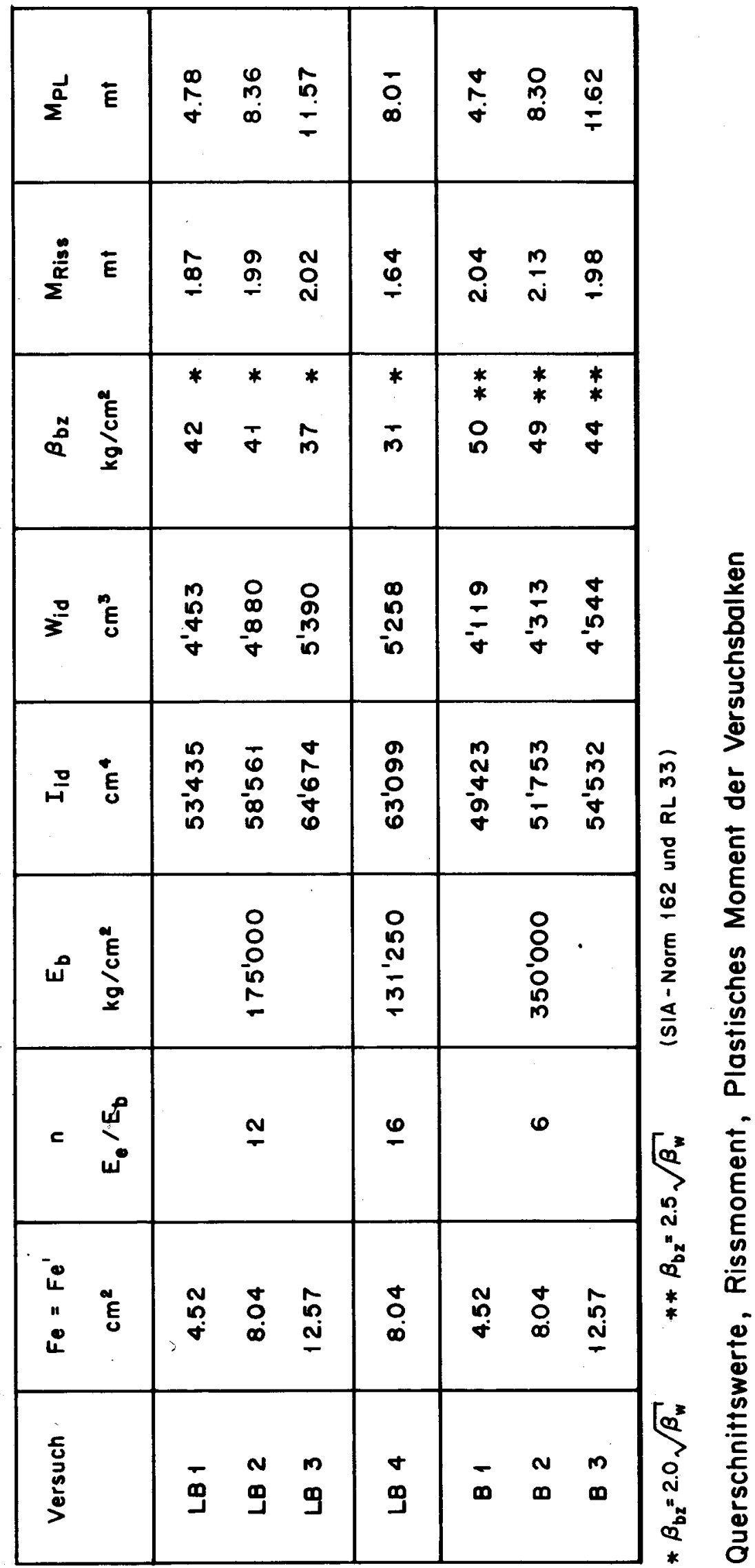




\begin{tabular}{|c|c|c|c|c|c|c|}
\hline \multirow{8}{*}{ 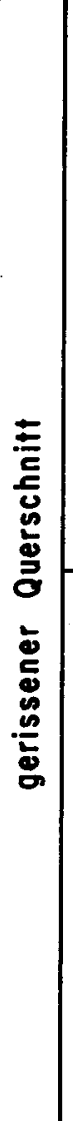 } & \multirow{4}{*}{ 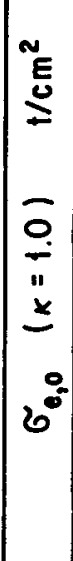 } & \multirow{2}{*}{ 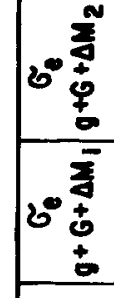 } & \multicolumn{2}{|c|}{ 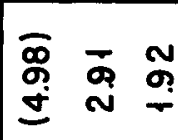 } & 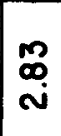 & 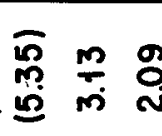 \\
\hline & & & $\begin{array}{l}\mathscr{L} \\
m \\
m \\
m\end{array}$ & $\frac{\dot{v}}{\dot{j}}$ & O̊ & 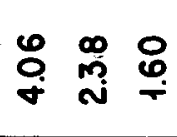 \\
\hline & & 2 & $\underset{N}{N}$ & $\stackrel{m}{m}{ }_{0}^{\infty}$ & $\stackrel{n}{n}$ & 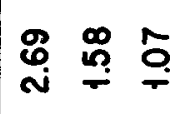 \\
\hline & & ఈ ० & $\begin{array}{l}\infty \\
0 \\
+\end{array}$ & 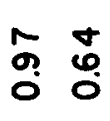 & 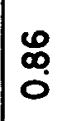 & 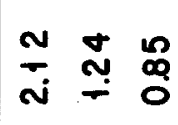 \\
\hline & 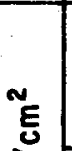 & 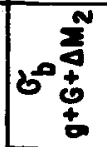 & $\underset{m}{m}$ & $\stackrel{\mathscr{N}}{\sim} \stackrel{\sim}{\circ}$ & 움 & 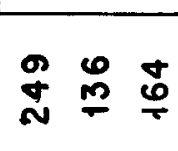 \\
\hline & $\Phi$ & 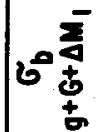 & & $m$ & $\stackrel{n}{R}$ & 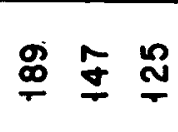 \\
\hline & రำ & 0 & $m$ & in $\varphi$ & $\dot{8}$ & $\underset{\sim}{\sim}$ \& \\
\hline & & م مా & is & $\mathcal{F}$ & ก & $\stackrel{\infty}{\infty} \underset{\boldsymbol{\sigma}}{\boldsymbol{\sigma}}$ \\
\hline $\bar{E}$ & Ẽ & ○章 & $\stackrel{n}{m}$ & $\underset{\infty}{\sim} \underset{\infty}{\sim}$ & $\begin{array}{l}\infty \\
\infty \\
n\end{array}$ & 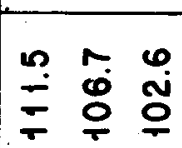 \\
\hline 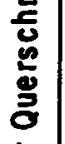 & 2 & o & $\begin{array}{ll}0 & 1 \\
\infty & 1 \\
0 & 1\end{array}$ & 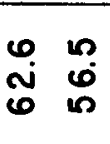 & $\begin{array}{l}\infty \\
\dot{\forall} \\
i\end{array}$ & 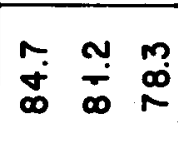 \\
\hline 总 & 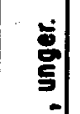 & $6 \begin{array}{l}0 \\
\vdots \\
0\end{array}$ &  & $\begin{array}{ll}n & N \\
\infty & \dot{\sigma} \\
m & m\end{array}$ & $\underset{\text { in }}{\dot{j}}$ & 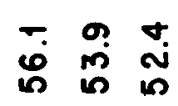 \\
\hline & סूँ & 100 & $\stackrel{9}{\circ}:$ & $\underset{\sim}{\stackrel{\infty}{\infty}}$ & $\stackrel{0}{\text { m }}$ & 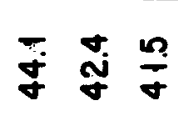 \\
\hline & 总 & z̃ & $\begin{array}{ll}\infty \\
\stackrel{N}{N} & \vdots\end{array}$ & 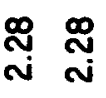 & 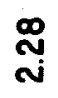 & 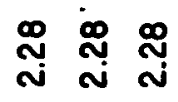 \\
\hline$\frac{5}{2}$ & & $\sum^{-}$ & $\stackrel{\infty}{\square}$ & $\stackrel{\infty}{\dddot{T}} \stackrel{\infty}{\square}$ & $\stackrel{\infty}{\dddot{\varphi}}$ & $\stackrel{\infty}{\leftarrow} \stackrel{\infty}{\longleftarrow} \stackrel{\infty}{\longleftarrow}$ \\
\hline & $\frac{\hat{6}}{\underline{E}}$ & 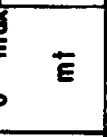 & $\underset{8}{9}$ & 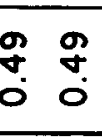 & $\underset{0}{0}$ & 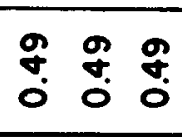 \\
\hline & $\frac{0}{\frac{E}{0}}$ & 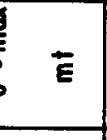 & $\stackrel{\infty}{\infty}$ & $\stackrel{m}{m} \stackrel{\infty}{m}$ & సָ & 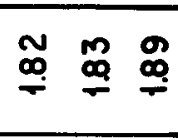 \\
\hline & & EE & $\stackrel{\infty}{\simeq} \stackrel{0}{=}$ & $\stackrel{\infty}{\stackrel{\infty}{T}}$ & $\underline{n}$ & 怘 \\
\hline & 5 & & $\overline{9}$ & $\begin{array}{ll}\sim & m \\
\infty & 9\end{array}$ & $\begin{array}{l}+ \\
9 \\
9\end{array}$ & 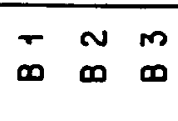 \\
\hline
\end{tabular}

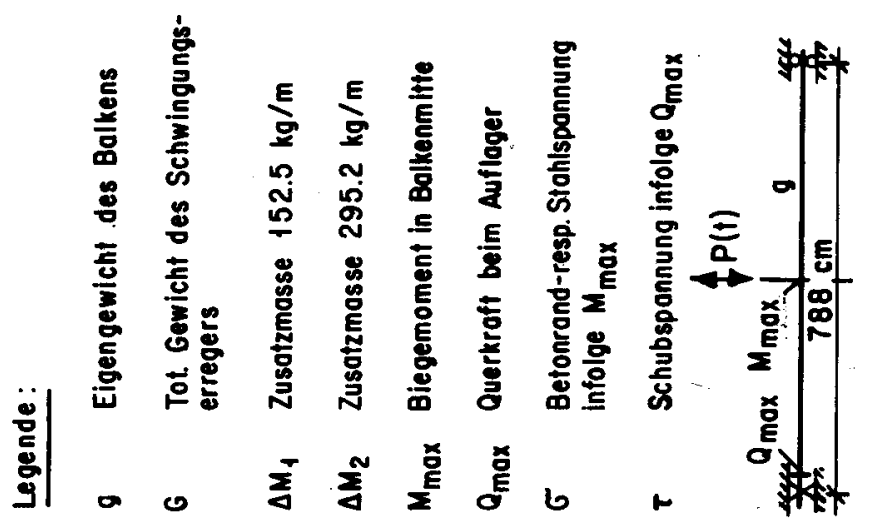

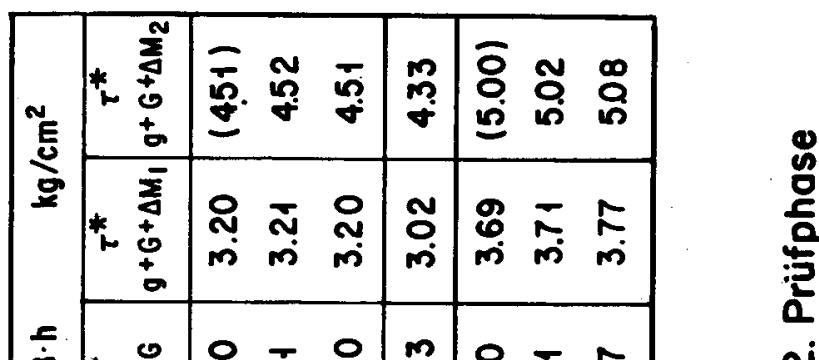

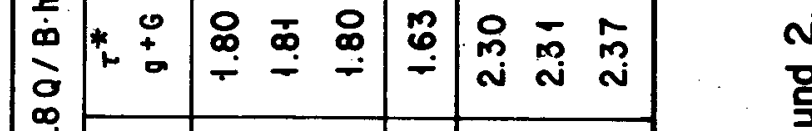

ma

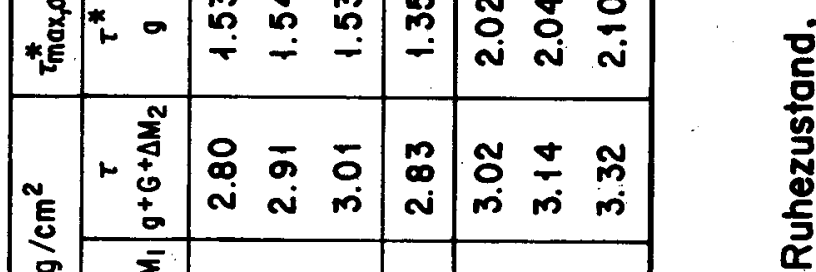

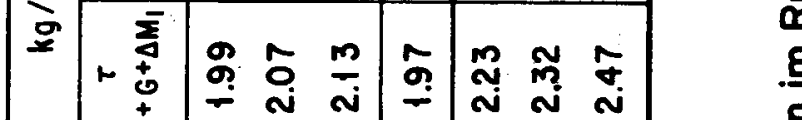

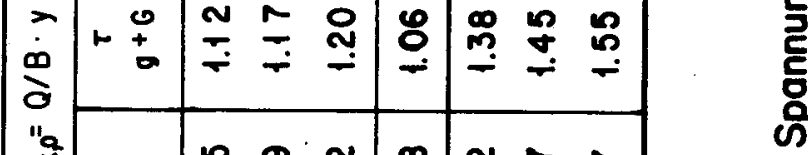

"

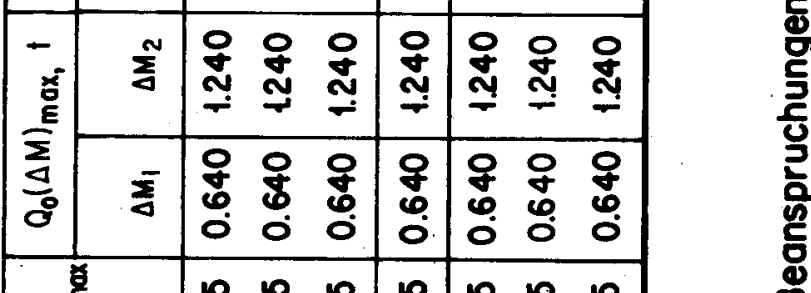

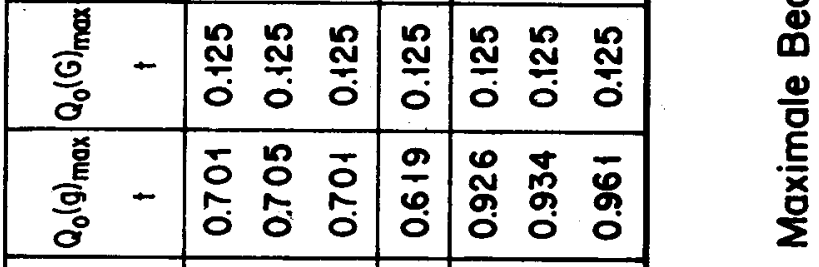

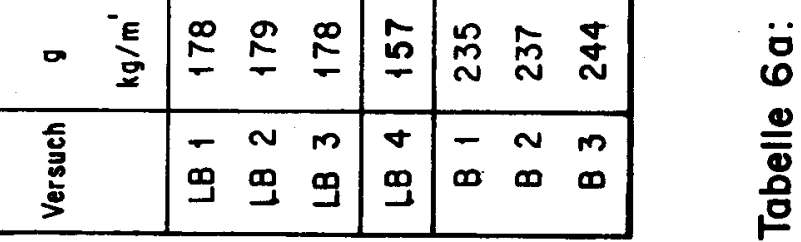



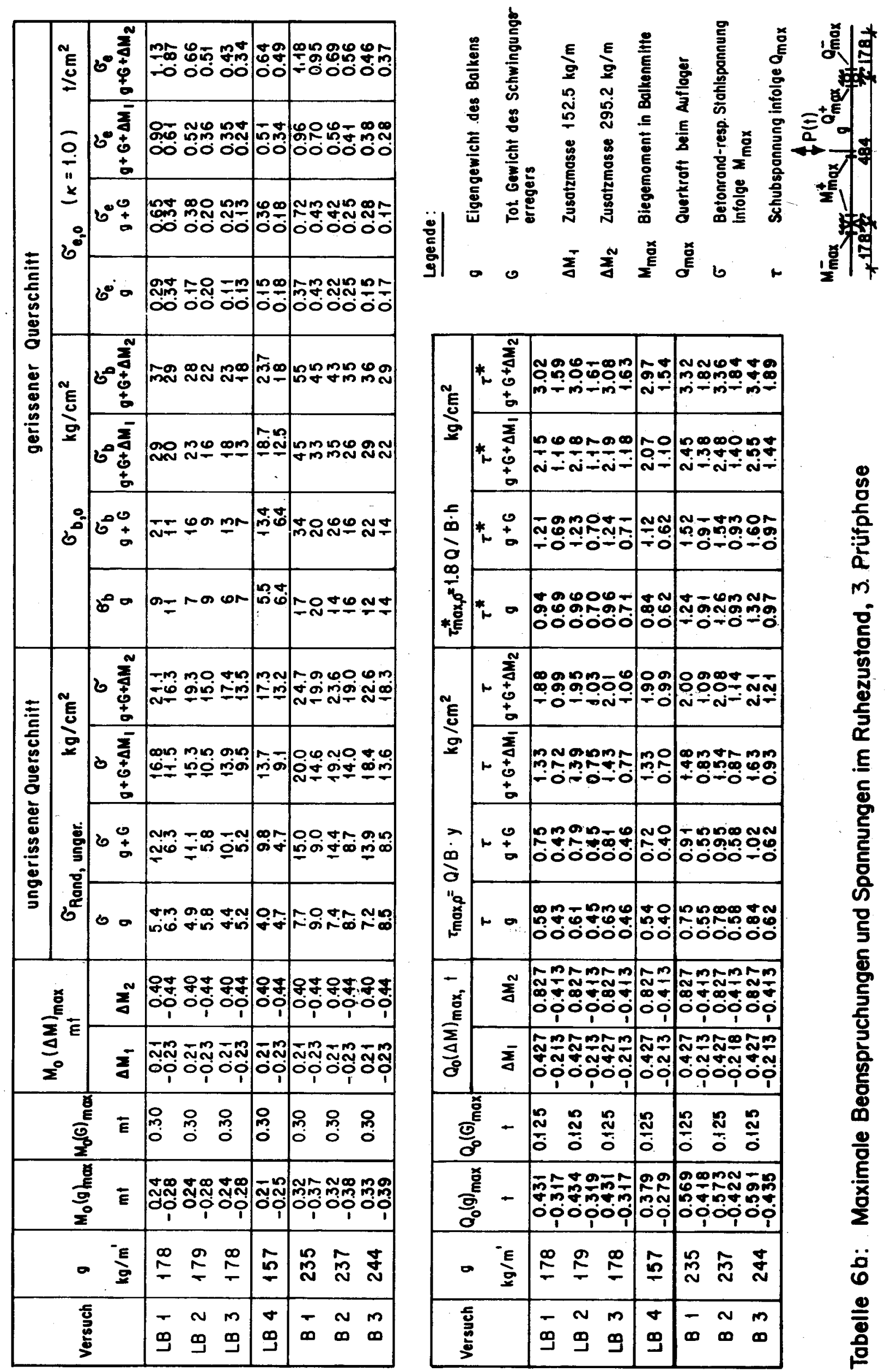

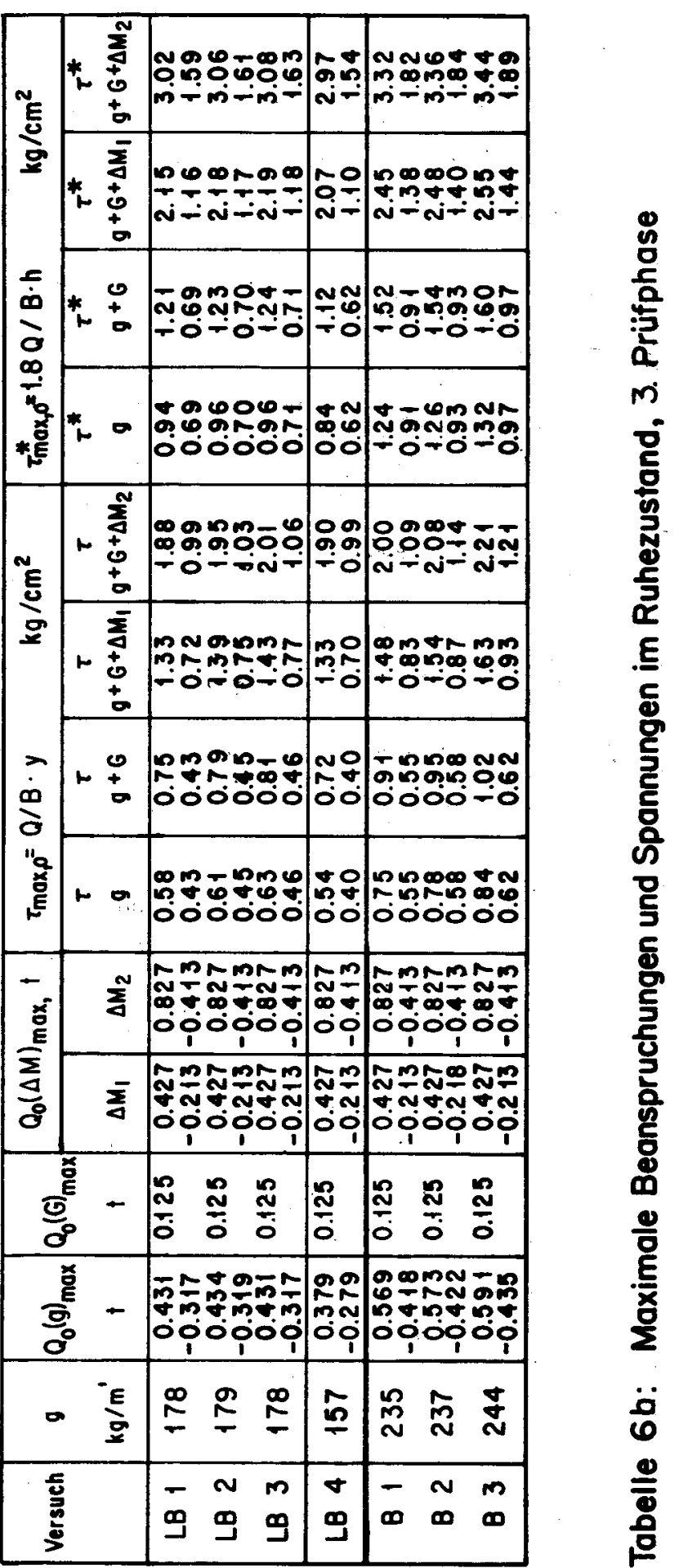




\begin{tabular}{|c|c|c|c|c|c|c|c|c|c|c|c|c|c|}
\hline \multirow[b]{2}{*}{ 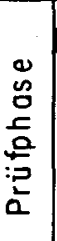 } & \multirow{2}{*}{$\begin{array}{l}\text { Beanspru- } \\
\text { chungs- } \\
\text { stufe }\end{array}$} & \multirow{2}{*}{$\sigma_{e, a b s}$} & \multirow{2}{*}{$\Delta \mathrm{M}$} & \multirow[b]{2}{*}{$\begin{array}{l}\text { totale } \\
\text { Mosse } \\
{[\mathrm{kg} / \mathrm{m}]}\end{array}$} & \multirow[b]{2}{*}{$\begin{array}{l}\text { Stütz- } \\
\text { werte } \\
1 \\
{[\mathrm{~m}]}\end{array}$} & \multirow[b]{2}{*}{$\begin{array}{c}M_{0} \\
{[m t]}\end{array}$} & \multirow[b]{2}{*}{$\begin{array}{c}G_{\mathrm{e}, 0} \\
{\left[\mathrm{t} / \mathrm{cm}^{2}\right]}\end{array}$} & \multirow[b]{2}{*}{$\begin{array}{l}M_{\text {tel }} \\
{[\mathrm{mt}]}\end{array}$} & \multirow{2}{*}{$\begin{array}{l}\sigma_{e, \text { rel }} \\
{\left[t / \mathrm{cm}^{2}\right]}\end{array}$} & \multicolumn{2}{|c|}{ Ausschwingversuch } & \multicolumn{2}{|c|}{ Resonanzversuch } \\
\hline & & & & & & & & & & $\begin{array}{l}P_{A} \\
{[\mathrm{~kg}]}\end{array}$ & $\begin{array}{c}* \\
\delta_{\max , r e} \\
{[\mathrm{~cm}]}\end{array}$ & $\begin{array}{c}* \\
\hat{\rho}_{\max , \mathrm{rel}} \\
{[\mathrm{cm}]}\end{array}$ & $\begin{array}{c}* \\
\text { Eigenfre- } \\
\text { quenz } f \\
{[t / s e c]}\end{array}$ \\
\hline 1 & 2 & 3 & 4 & 5 & 6 & 7 & 8 & 9 & 10 & 11 & 12 & 13 & 14 \\
\hline \multirow[t]{15}{*}{1} & $1.5-00-1$ & 15 & 0 & 179 & 7.88 & 1.88 & 1.31 & 0.27 & 0.19 & 137 & 0.32 & 0.44 & 3.40 \\
\hline & 2.00 .01 & 2.0 & 0 & 179 & 7.88 & 1.88 & 1.31 & 0.99 & 0.69 & 502 & 4.12 & 4.52 & 3.40 \\
\hline & $2.5-0.0-1$ & 2.5 & 0 & 179 & 7.88 & 1.88 & 1.31 & 1.70 & 1.19 & 863 & 1.91 & 2.60 & 3.40 \\
\hline & $2.5-0.5-1$ & & 152.4 & 331.4 & & 3.06 & 2.14 & 0.51 & 0.36 & 259 & 0.62 & 0.84 & 2.64 \\
\hline & $3.0-0.5-1$ & 3.0 & 152.4 & 331.4 & 7.88 & 3.06 & 2.14 & 1.23 & 0.86 & 624 & 1.42 & 1.93 & 2.64 \\
\hline & $3.0-0.0-1$ & & 0 & 0 & & 1.88 & 1.31 & 2.41 & 1.69 & 1223 & $2.7 d$ & 3.69 & 3.40 \\
\hline & $3.5-0.0-1$ & 3.5 & 0 & 0 & 7.88 & 4.88 & 1.31 & 3.13 & 2.19 & 1589 & 3.51 & 4.77 & 3.40 \\
\hline & $3.5-0.5-1$ & & 152.4 & 331.4 & & 3.06 & 2.14 & 1.94 & 1.36 & 985 & 2.22 & 3.03 & 2.64 \\
\hline & $3.5-1.0-1$ & & 295.2 & 474.2 & & 4.16 & 2.94 & 0.84 & 0.59 & 426 & 0.99 & 1.34 & 2.26 \\
\hline & $4.0-10-1$ & 4.0 & 295.2 & 474.2 & 7.88 & 4.16 & 2.91 & 1.56 & 1.09 & 792 & 1.79 & 2.44 & 2.36 \\
\hline & $40-0.5-1$ & & 152.4 & 331.4 & & 3.06 & 2.14 & 266 & 1.86 & 1350 & 3.02 & 4.11 & 2.64 \\
\hline & $4.0-0.0-1$ & & 0 & 0 & & 188 & 1.31 & 3.84 & 2.69 & 1949 & 4.32 & 5.87 & 3.40 \\
\hline & $4.5-0.0-1 \mid$ & 4.5 & 0 & 0 & 7.88 & 1.88 & 1.31 & 456 & 3.19 & $2 ' 315$ & 5.11 & 6.96 & 3.40 \\
\hline & $4.5-0.5-1$ & & 1524 & 331.4 & & 3.06 & 2.14 & 3.37 & 2.36 & 1'711 & 3.82 & 5.25 & 2.64 \\
\hline & $4.5-1.0-1$ & & 295.4 & 474.2 & & 4.16 & 2.91 & 2.27 & 1.59 & $1^{\prime} 152$ & 2.59 & 3.52 & 2.26 \\
\hline \multirow[t]{9}{*}{2} & $1.5-0.0-2$ & 4.5 & 0 & 0 & 7.88 & 1.88 & 1.31 & 027 & 0.19 & 137 & 0.32 & 0.44 & 3.40 \\
\hline & $2.5-0.0-2$ & 2.5 & 0 & 0 & 7.88 & 1.88 & 1.31 & 1.70 & 1.19 & 863 & 1.91 & 2.60 & 3.40 \\
\hline & 2.5-0.5-2 & & 152.4 & 331.4 & & 3.06 & 2.14 & 0.51 & 0.36 & 259 & 0.62 & 0.84 & 2.64 \\
\hline & $|3.5-0.0-2|$ & 3.5 & 0 & 0 & 7.88 & 1.88 & 1.31 & 3.13 & 2.19 & $1^{\prime} 589$ & 3.51 & 4.77 & 3.40 \\
\hline & $3.5-0.5-2$ & & 152.4 & 3314 & & 3.06 & 2.14 & 1.94 & 1.36 & 985 & 2.22 & 3.03 & 2.64 \\
\hline & $3.5-1.0-2$ & & 295.2 & 474.2 & & 4.16 & 2.91 & 0.84 & 0.59 & 426 & 0.99 & 1.34 & 2.26 \\
\hline & $45-1.0-2$ & 4.5 & 295.2 & 474.2 & 7.88 & 4.16 & 291 & 2.27 & 1.59 & $f^{\prime} 152$ & 2.59 & 3.52 & 2.26 \\
\hline & $4.5-0.5-2$ & & 152.4 & 331.4 & & 3.06 & 2.14 & 3.37 & 2.36 & 1711 & 3.82 & 5.25 & 2.64 \\
\hline & $4.5-0.0-2$ & & 0 & 0 & & 188 & 1.31 & 4.56 & 3.19 & 2315 & 5.11 & 6.96 & 3.40 \\
\hline \multirow[t]{9}{*}{3} & $0.5-0.0-3$ & 0.5 & 0 & 0 & 4.84 & 0.54 & 0.38 & 0.17 & 0.12 & 140 & 0.08 & 0.11 & 7.55 \\
\hline & $1.5 \cdot 0.0-3$ & 1.5 & 0 & 0 & 4.84 & 0.54 & 0.38 & 1.60 & 1.12 & $1^{\prime} 322$ & 0.68 & 0.90 & 7.55 \\
\hline & $1.5-1.0-3$ & & 295.2 & 474.2 & & 0.94 & 0.66 & 1.20 & 0.84 & 992 & 0.53 & 0.70 & 5.02 \\
\hline & $2.5-1.0-3$ & 2.5 & 295.2 & 474.2 & 4.84 & 0.84 & 0.66 & 2.63 & 1.84 & $2^{\prime} 174$ & 1.23 & $t .48$ & 5.02 \\
\hline & $2.5-0.0-3$ & & 0 & 0 & & 0.54 & 0.38 & 3.03 & 2.12 & 2'504 & 1.29 & 1.69 & 7.55 \\
\hline & $3.5-0.0-3$ & 3.5 & 0 & 0 & 4.84 & 0.54 & 0.38 & 4.46 & 3.12 & $3^{\prime} 686$ & 1.89 & 2.48 & 7.55 \\
\hline & $3.5-1.0-3$ & & 295.2 & 474.2 & & 0.94 & 0.66 & 4.06 & 2.84 & 3355 & 1.73 & 2.28 & 5.02 \\
\hline & $4.5-1.0-3$ & 4.5 & 295.2 & 474.2 & 4.84 & 4.94 & 0.66 & 5.49 & 3.84 & $4^{\prime} 537$ & 2.33 & 3.07 & 5.02 \\
\hline & $4.5-0.0-3$ & & 0 & 0 & & 0.54 & 0.38 & 5.89 & 4.12 & $4^{\prime} 868$ & 2.49 & 3.27 & 7.55 \\
\hline
\end{tabular}

* gerechnet am gerissenen Querschnitt

rechnerische Werte ermittelt mit $E_{L B}=215.000 \mathrm{~kg} / \mathrm{cm}^{2}$

Tabelle 7: Prüfprogramm des Balkens LB 2 


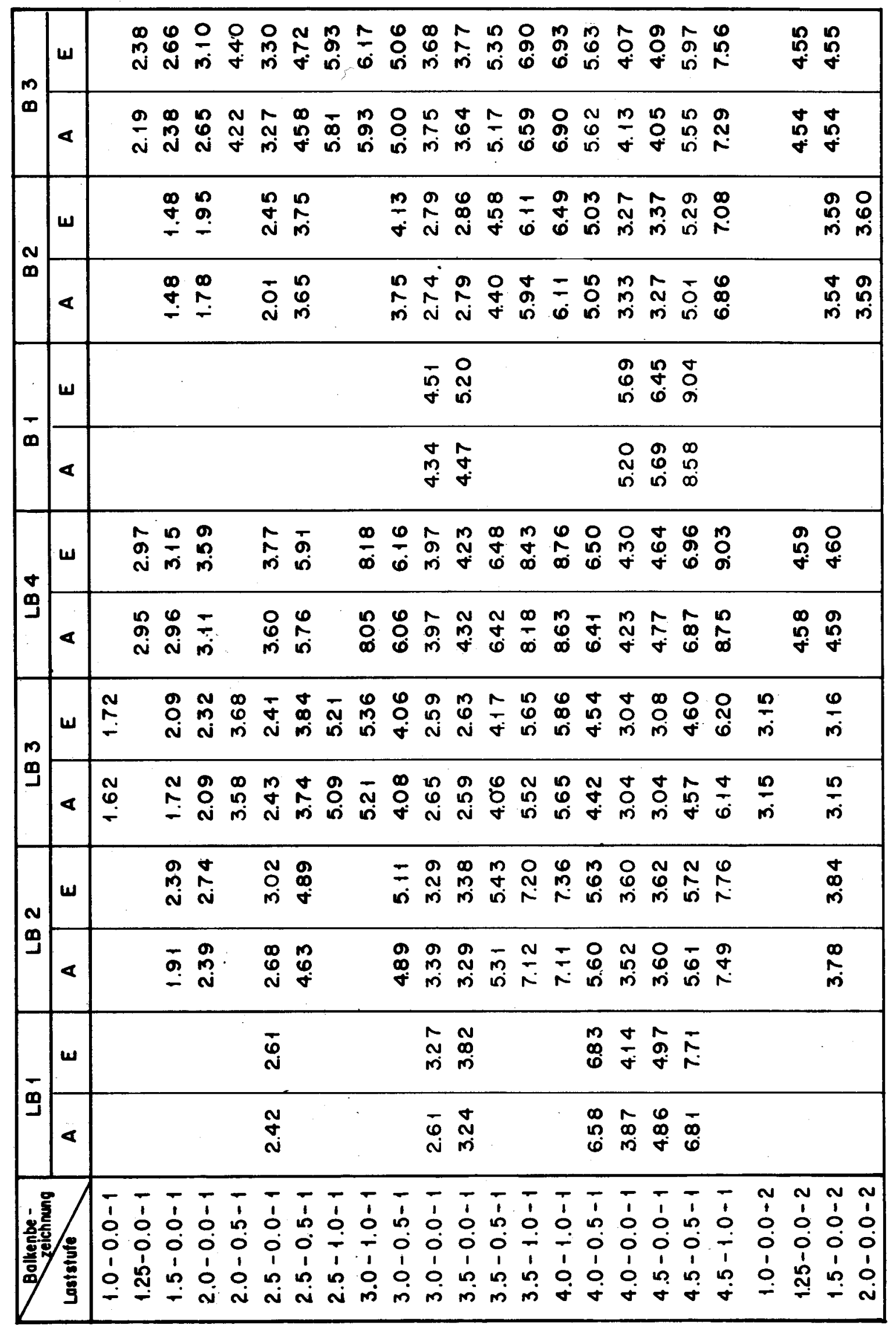




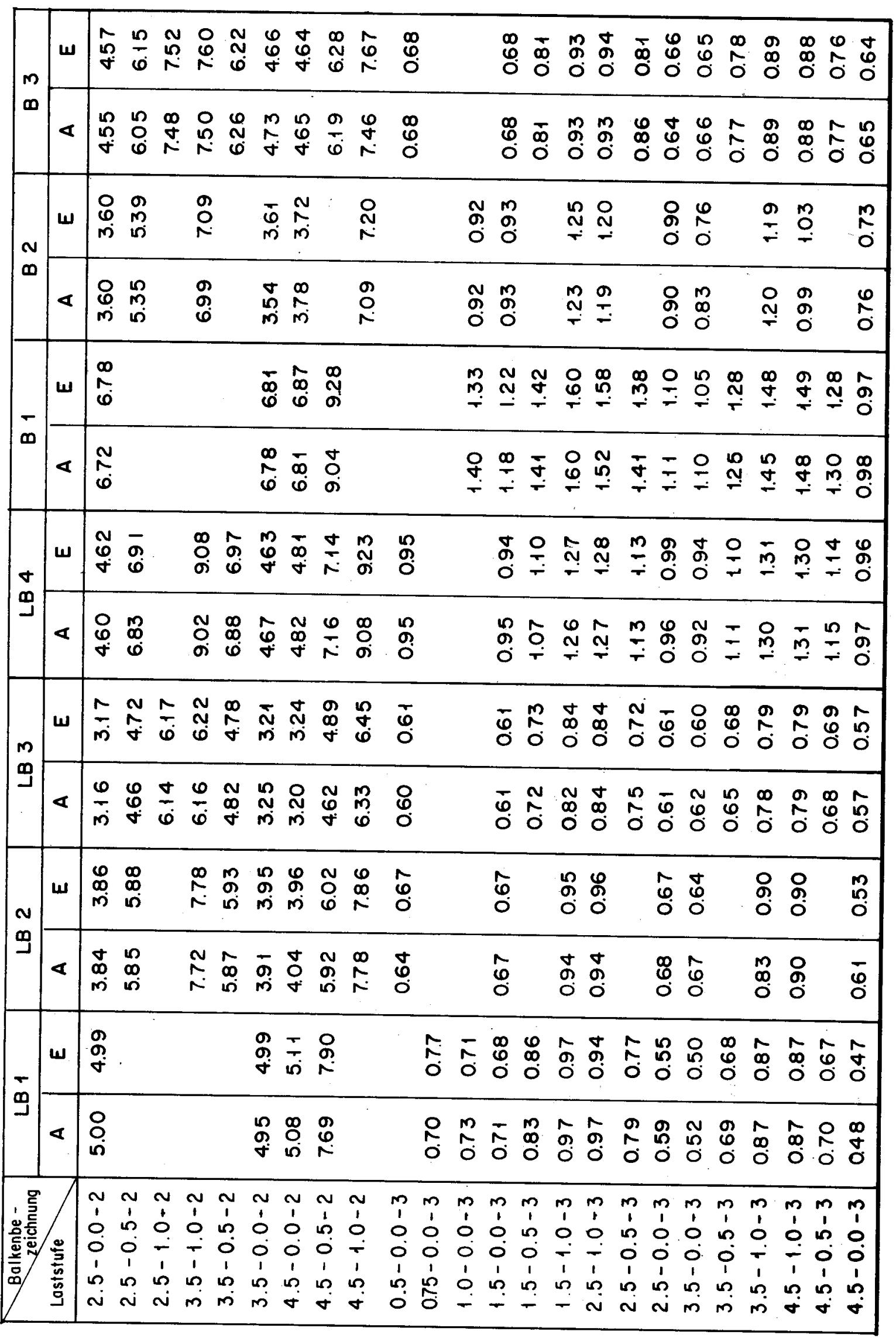

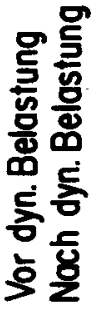
نن 


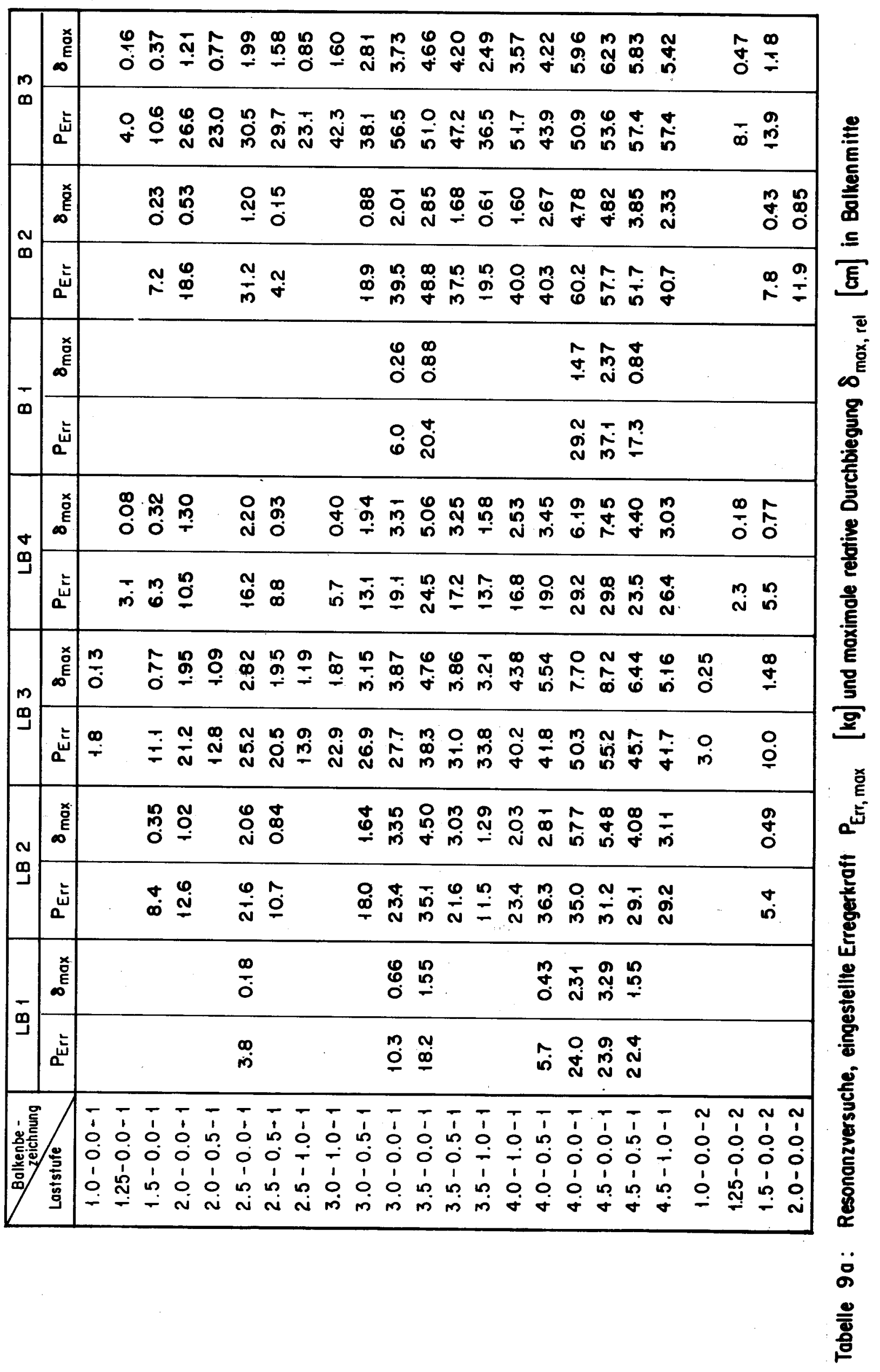




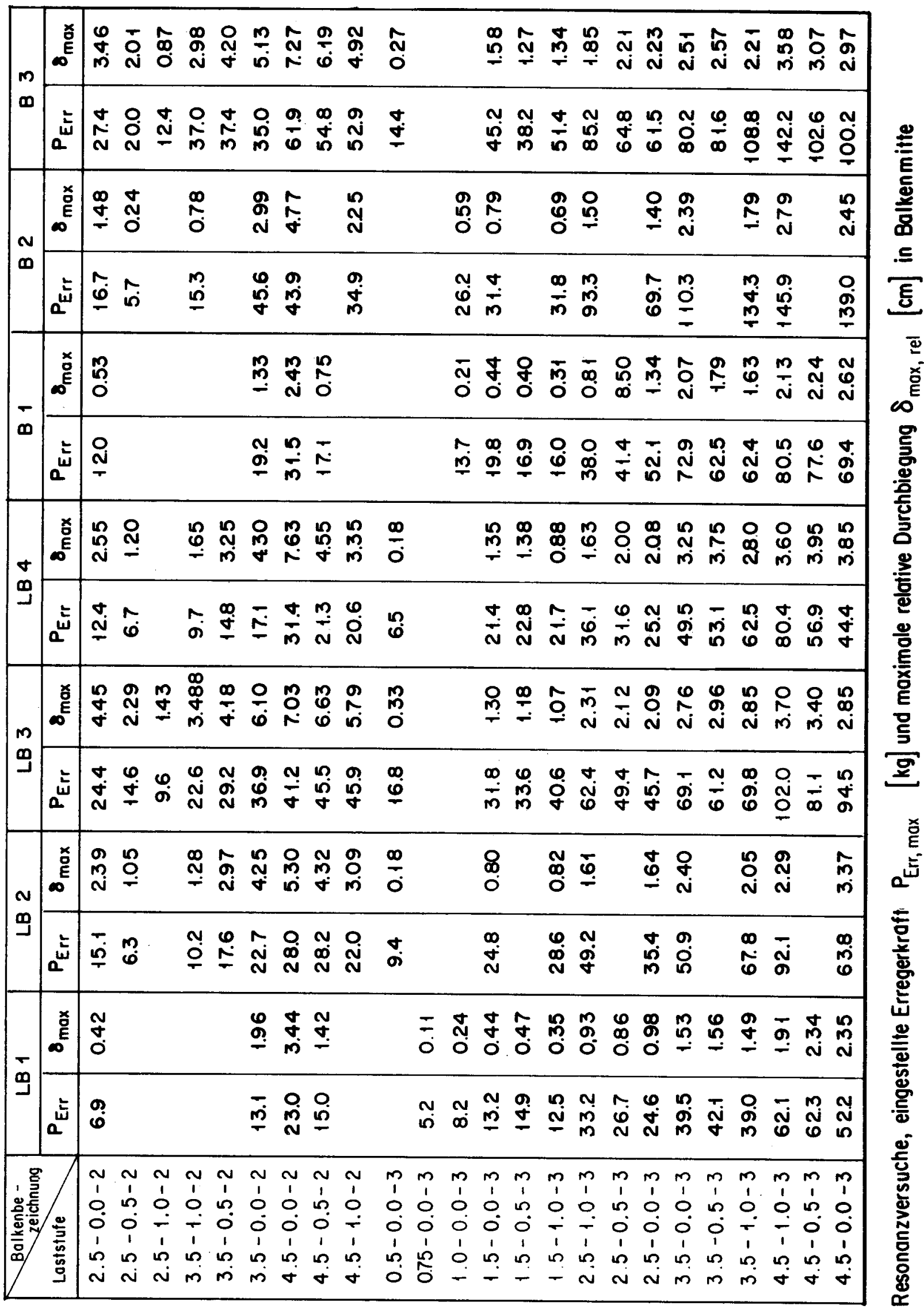




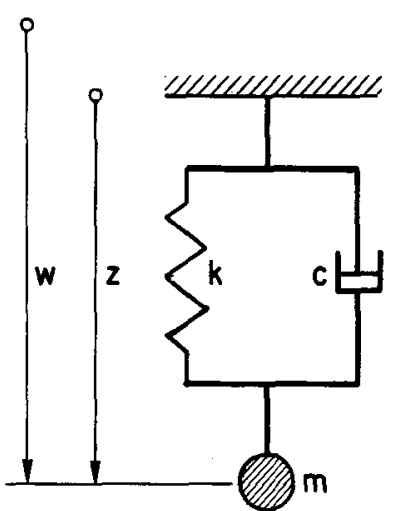

Bild 1: Modell eines Einmassenschwingers

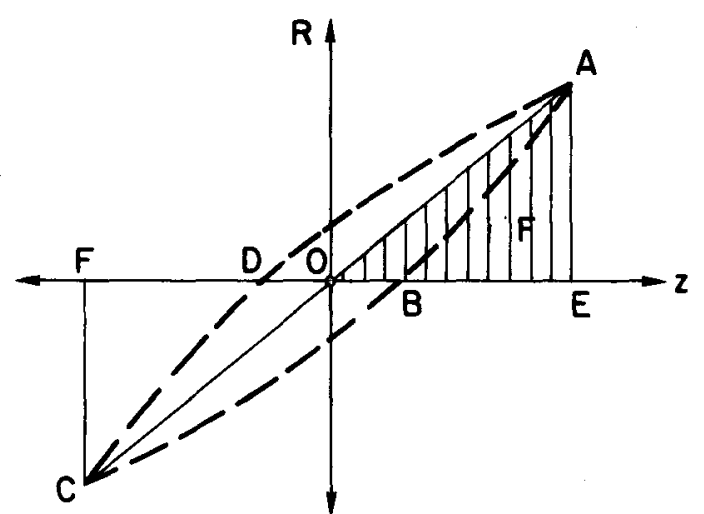

Bild 3: Einmassenschwinger mit Hysteresis Dämpfung

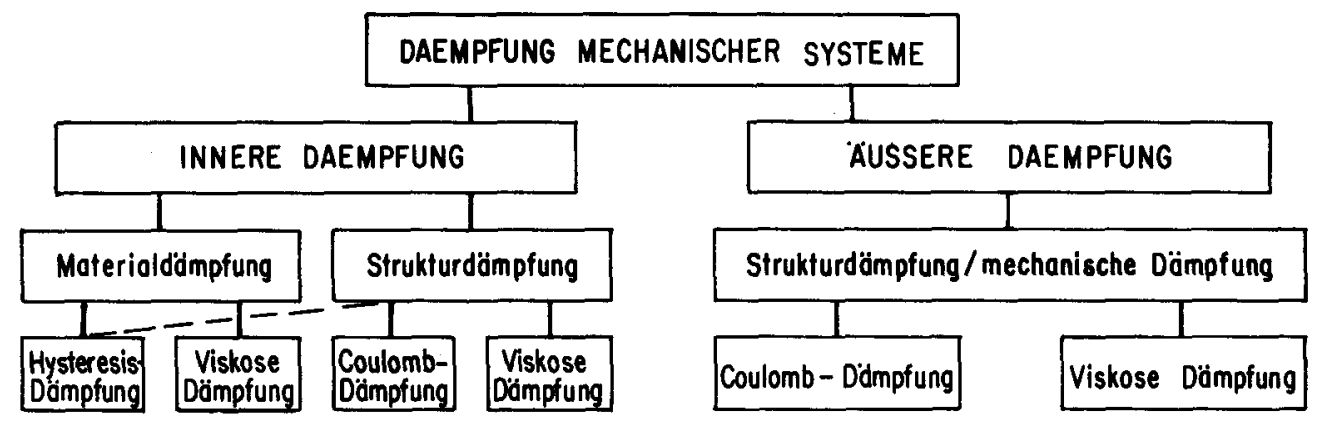

Bild 2: Klassierung der Dämpfung mechanischer Systeme

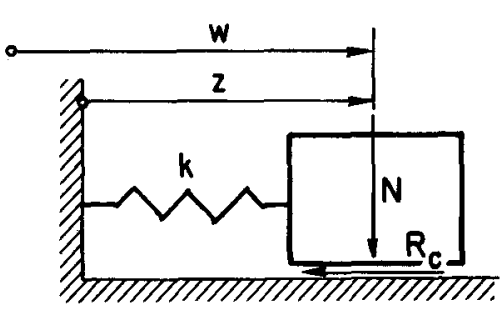

Bild 4: Modell eines Einmassenschwingers mit Coulomb-Dämpfung

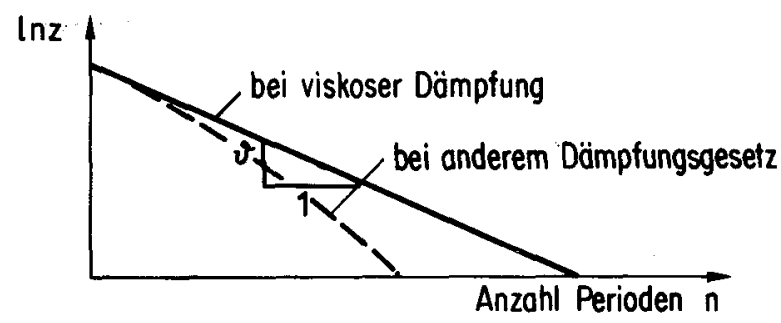

Bild 6: Kontrolle des Modells der viskosen Dämpfung

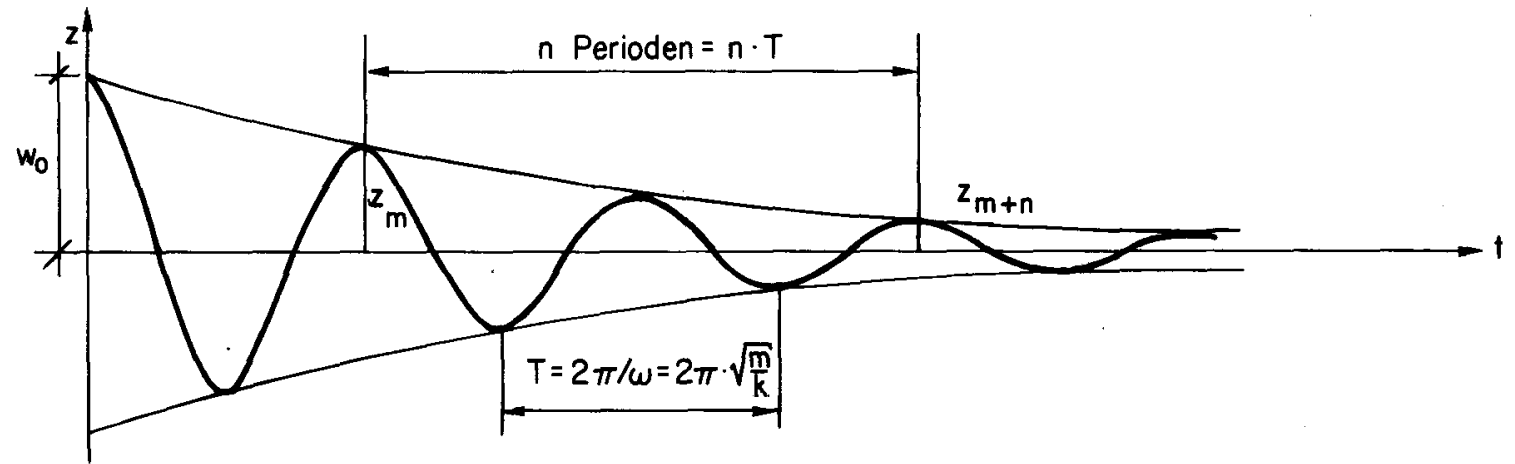

Bild 5: Amplitudenverlouf beim Ausschwingversuch 


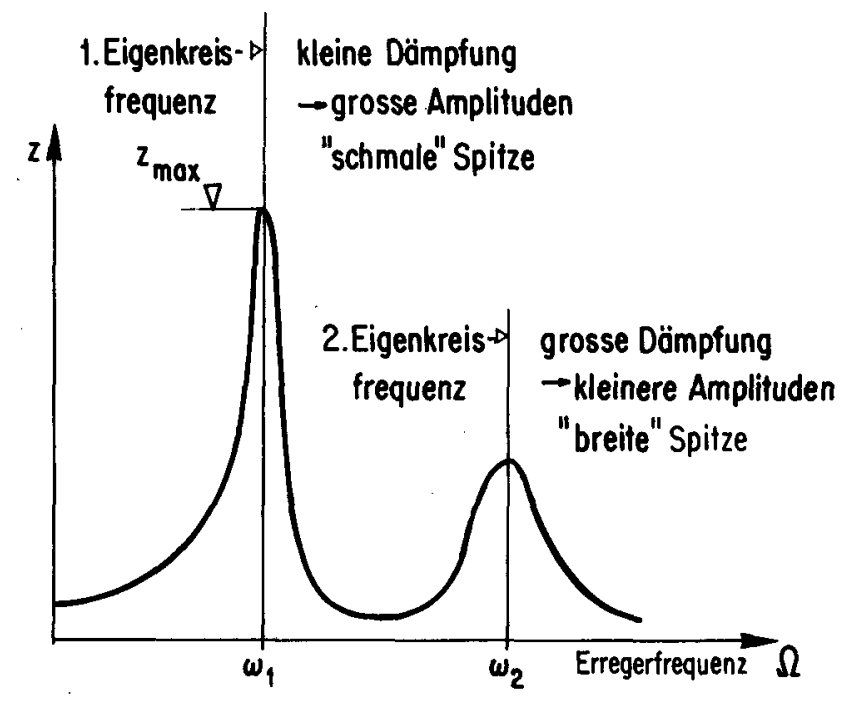

Bild 7 : Resonanzkurve eines Zwei-MossenSchwingers

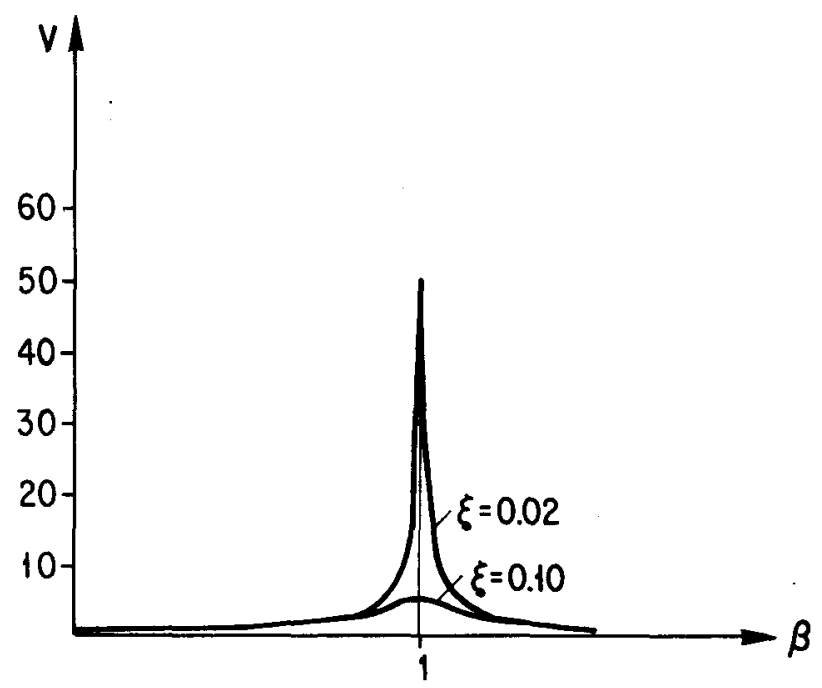

Bild 9 : Resonanzkurren bei verschiedenen Dämpfurigsmassen $\xi$

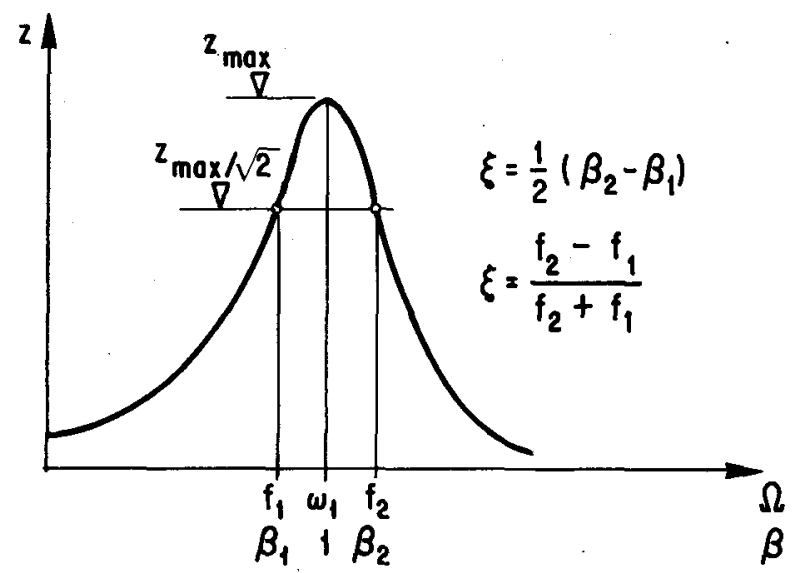

Bild 11: Methode der halben Bandbreite

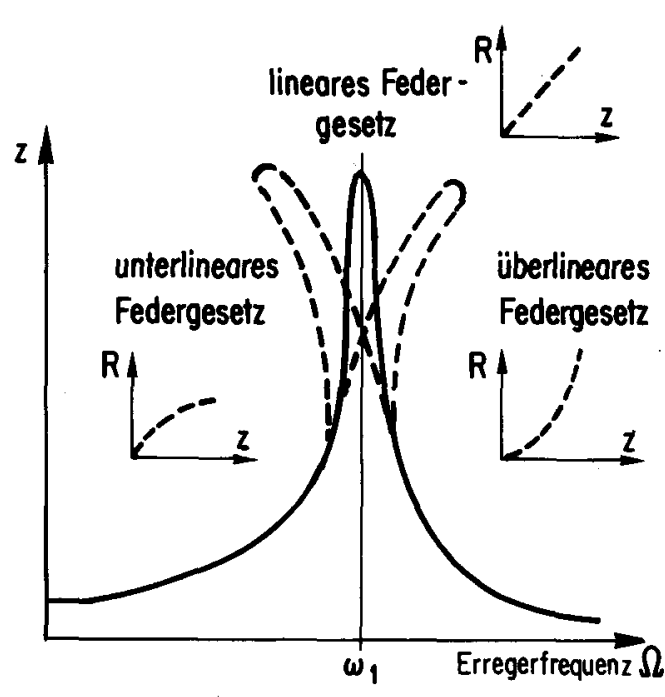

Bild 8 : Resonanzkurve eines Einmassenschwingers bei verschiedenen Federgesetzen

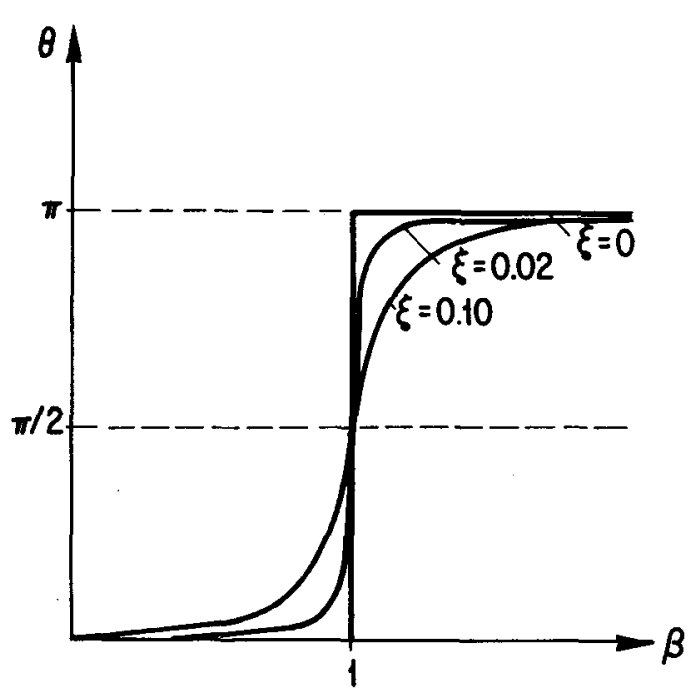

Bild 10 : Phasenverschiebungen bei verschiedenen Dömpfungsmassen $\xi$

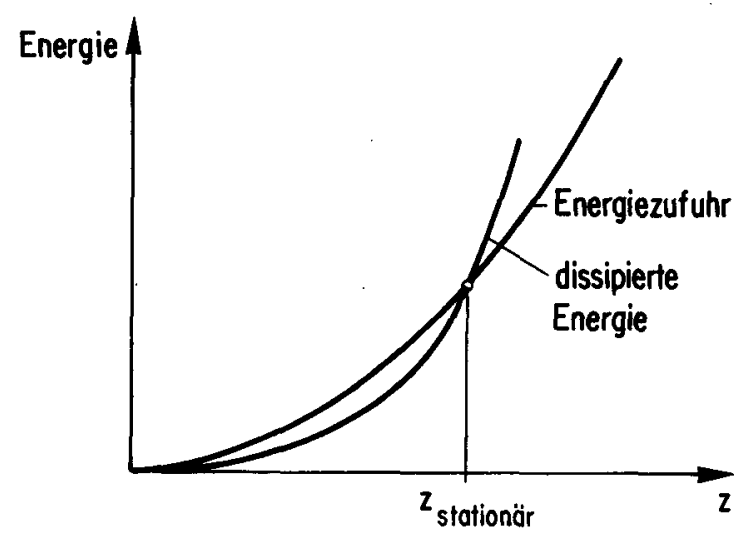

Bild 12 : Energetischer Zustand bei einer erzwungenen Schwingung 


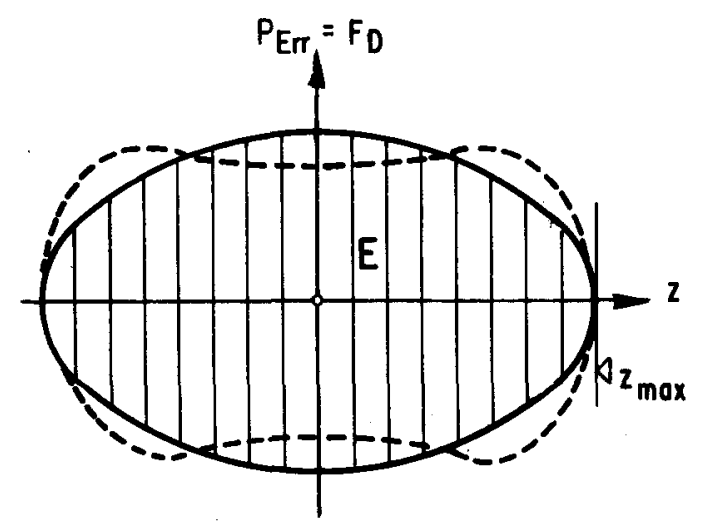

Bild 13: Kroft-Verschiebungs-Diagramm bei Resonanz

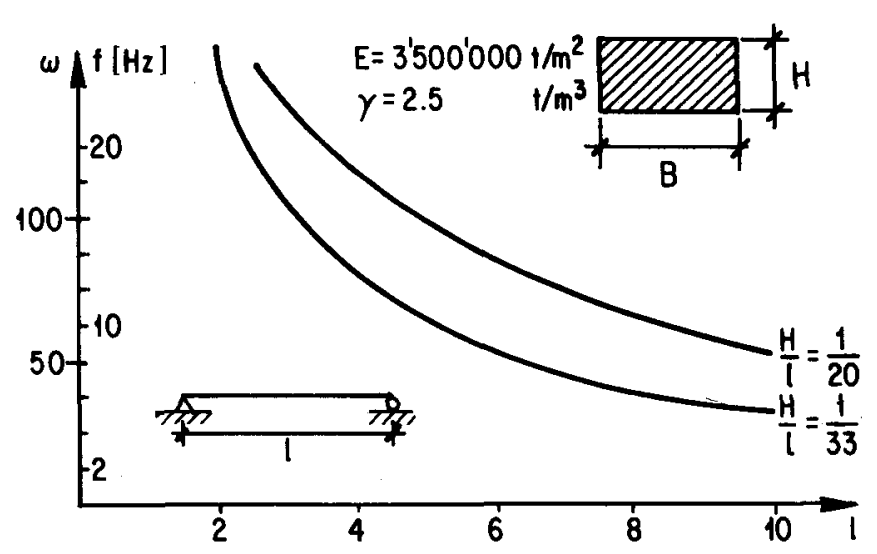

Bild 15 : Eigenfrequenzen am einfachen Balken

\begin{tabular}{|ll|c|c|c|c|c|}
\hline & & $v$ & $d$ & $\xi$ & $\psi$ & $c$ \\
\hline Logarithmisches Dekrement & $\sigma$ & 1 & $\pi \cdot d$ & $2 \pi \xi$ & $\psi / 2$ & $\pi \cdot c / \sqrt{k \cdot m}$ \\
Verlustfaktor & $d$ & $v / \pi$ & 1 & $2 \xi$ & $\psi / 2 \pi$ & $c \omega / k$ \\
Dömpfungsmoss & $\xi$ & $v / 2 \pi$ & $d / 2$ & 1 & $\psi / 4 \pi$ & $c / c_{\mathrm{kr}}=c / 2 \cdot \sqrt{\mathrm{k} \cdot \mathrm{m}}$ \\
Dömpfungskapazität & $\psi$ & $2 v$ & $2 \pi d$ & $4 \pi \xi$ & 1 & $2 \pi \mathrm{c} / \sqrt{\mathrm{k} \cdot \mathrm{m}}$ \\
Dömpfungskoeffizient & $c$ & $v \sqrt{\mathrm{k} \cdot \mathrm{m} / \pi}$ & $d \cdot k / \omega$ & $2 \xi \sqrt{\mathrm{k} \cdot \mathrm{m}}$ & $\sqrt{\mathrm{k} \cdot \mathrm{m}} \psi / 2 \cdot \pi$ & 1 \\
\hline
\end{tabular}

Bild 14 : Umrechnung der Dämpfungskenngrössen

1. und 2. Prüfphose

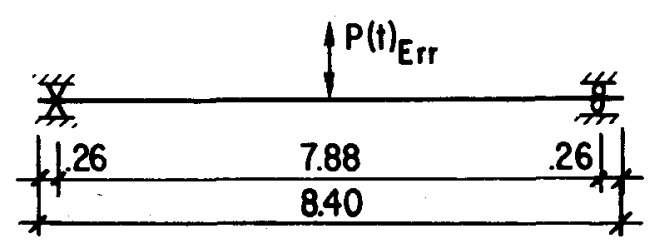

Bild 16 : Statische Systeme
3. Prüfphase

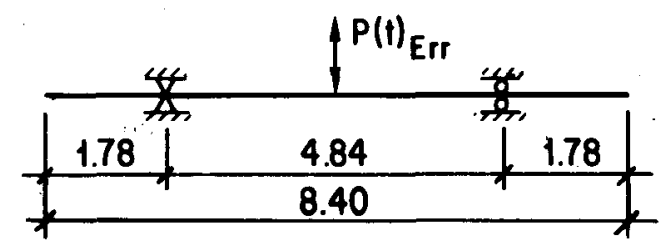

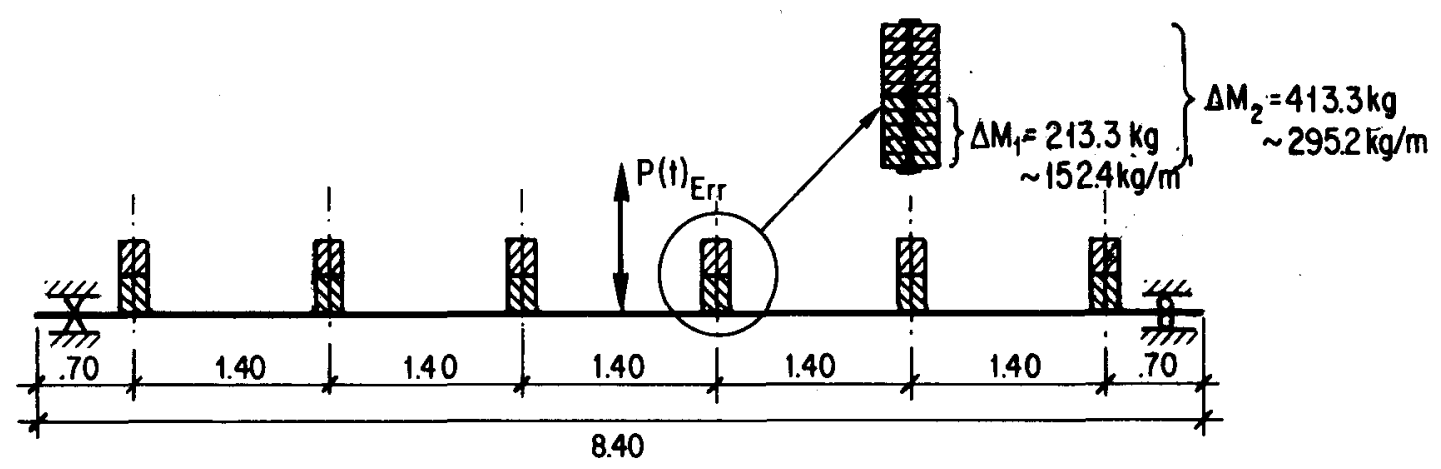

Bild 17 : Anordnung der Zusatzmassen 


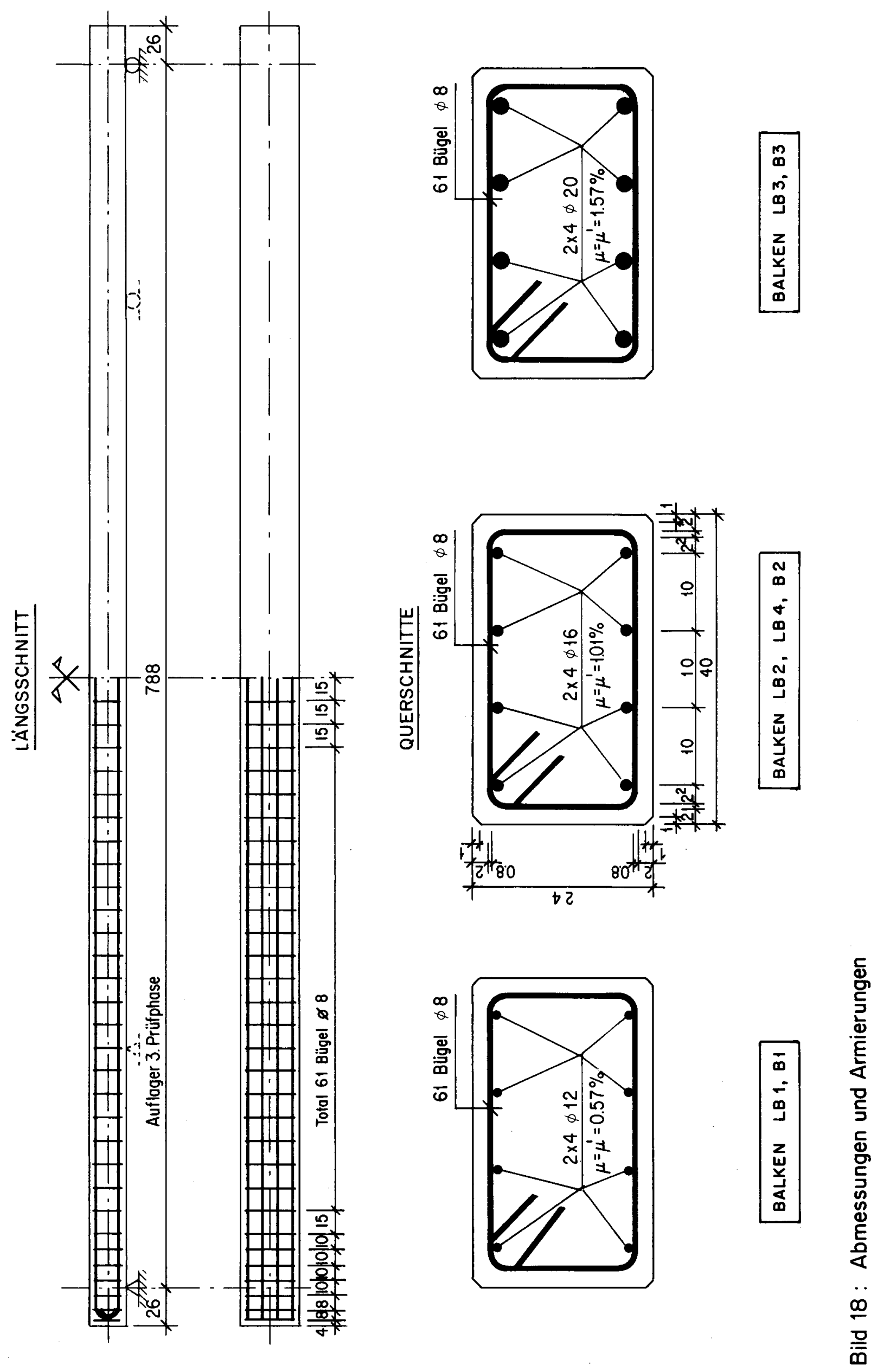



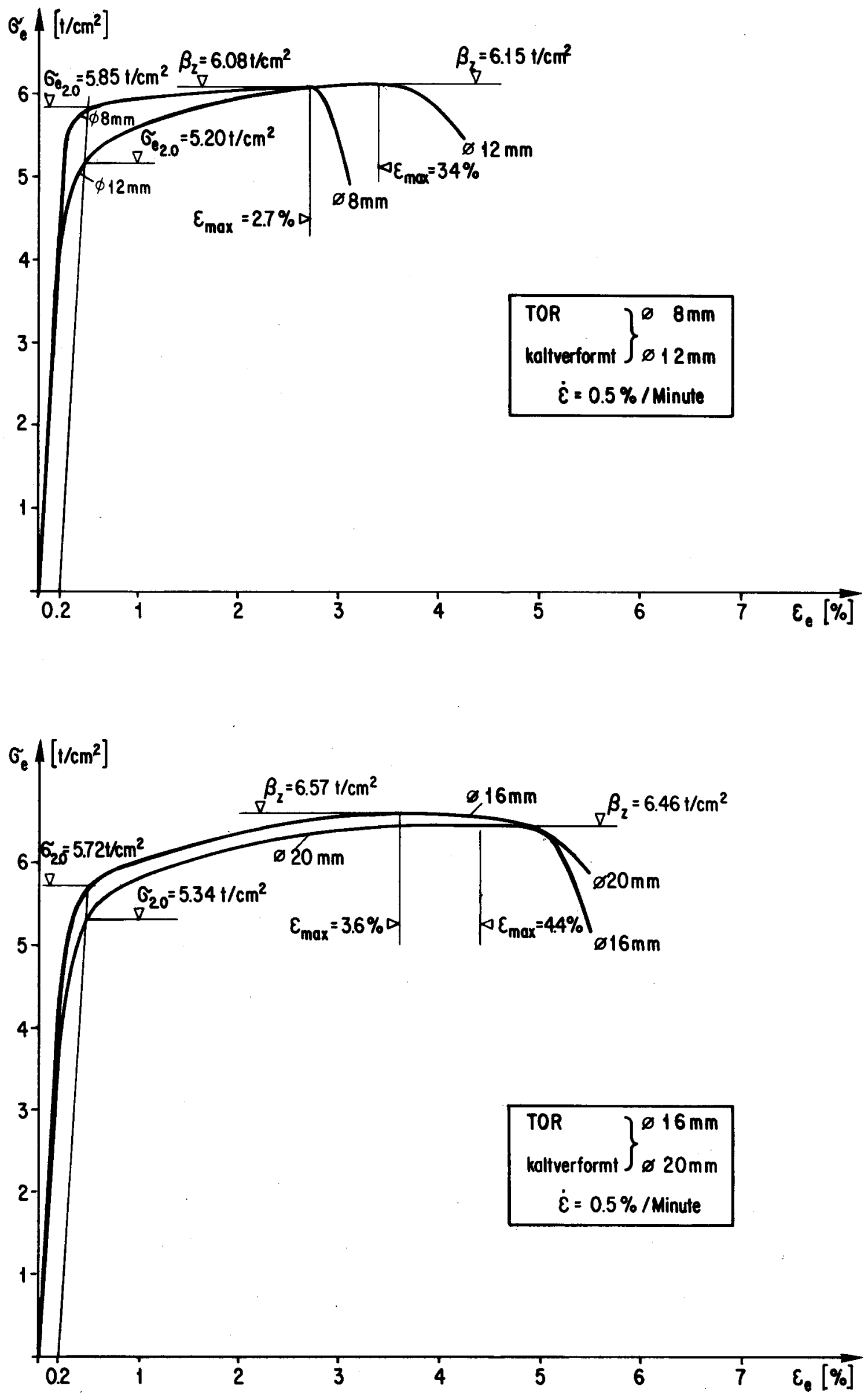

Bild 19: Spannungs-Dehnungs-Diagramme der verwendeten Armierungsstähle 


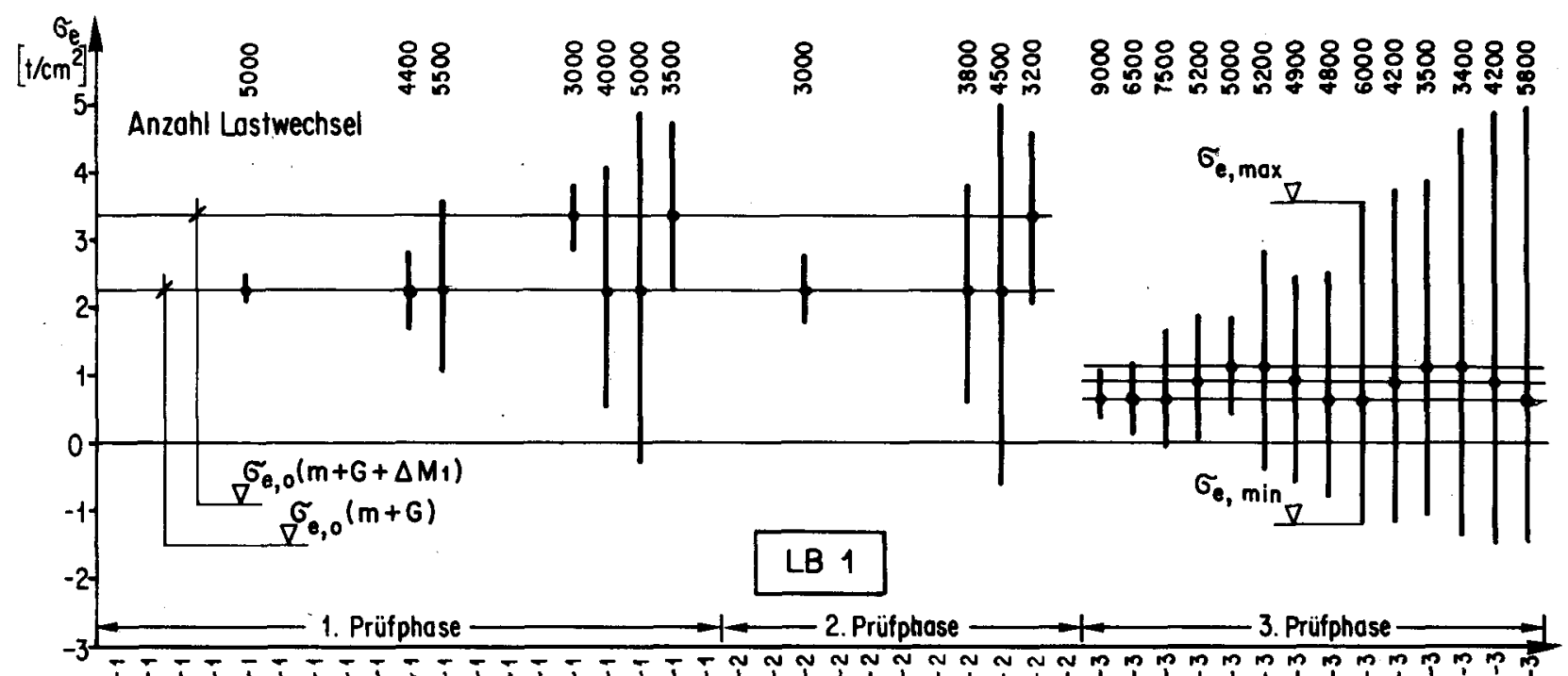

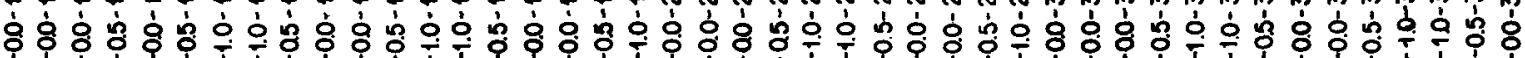

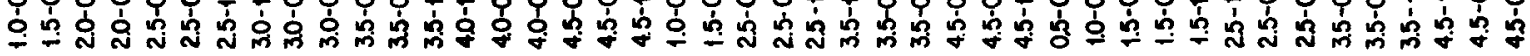
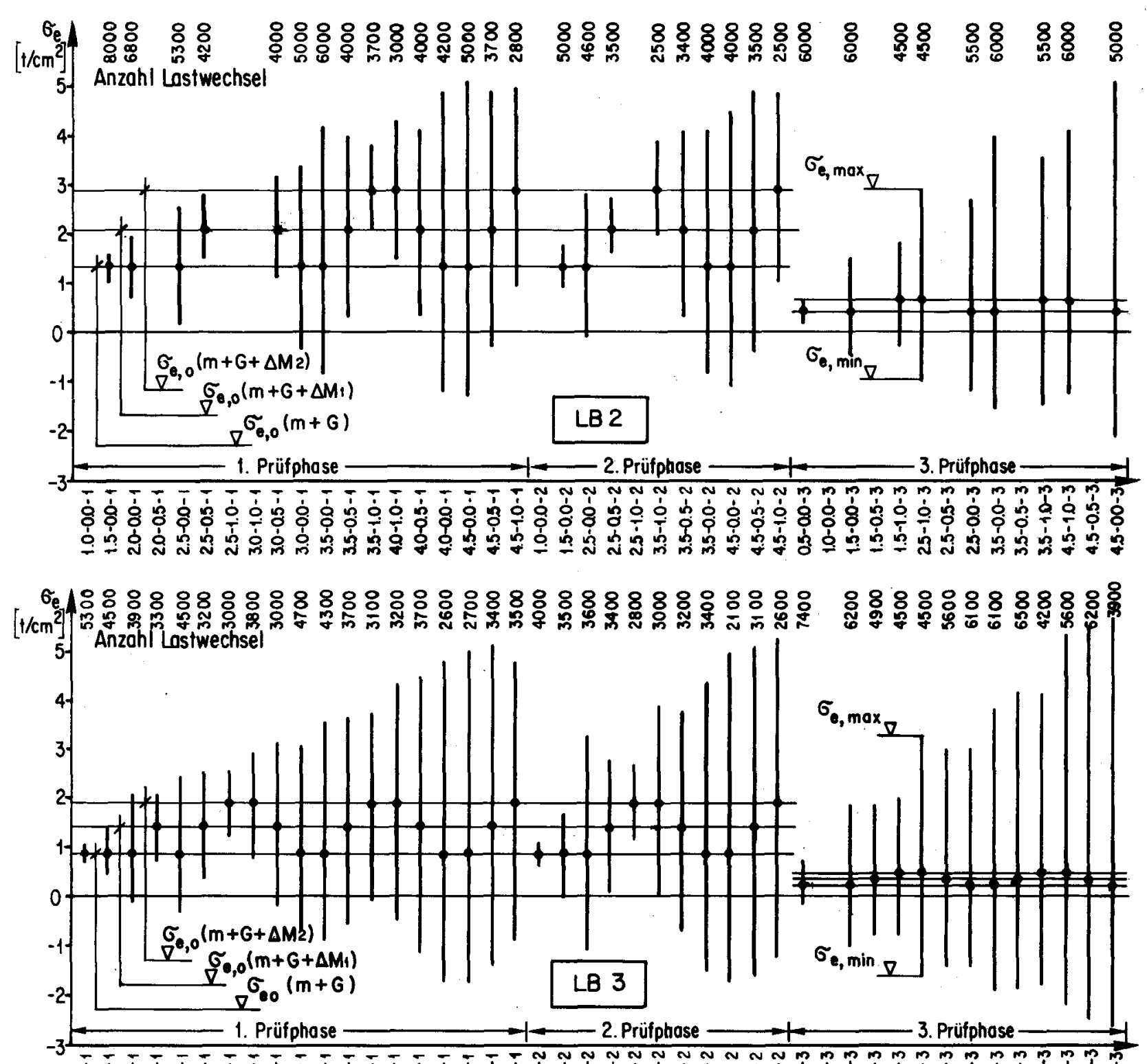

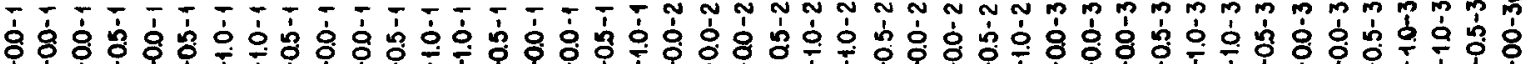

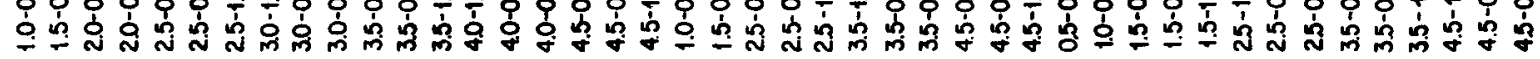

Bild 20a: Beanspruchung des Armierungsstahles, max. Schwingweiten und Lastwechsel je Beanspruchungsstufe der Balken LB 1, LB 2 und LB 3 

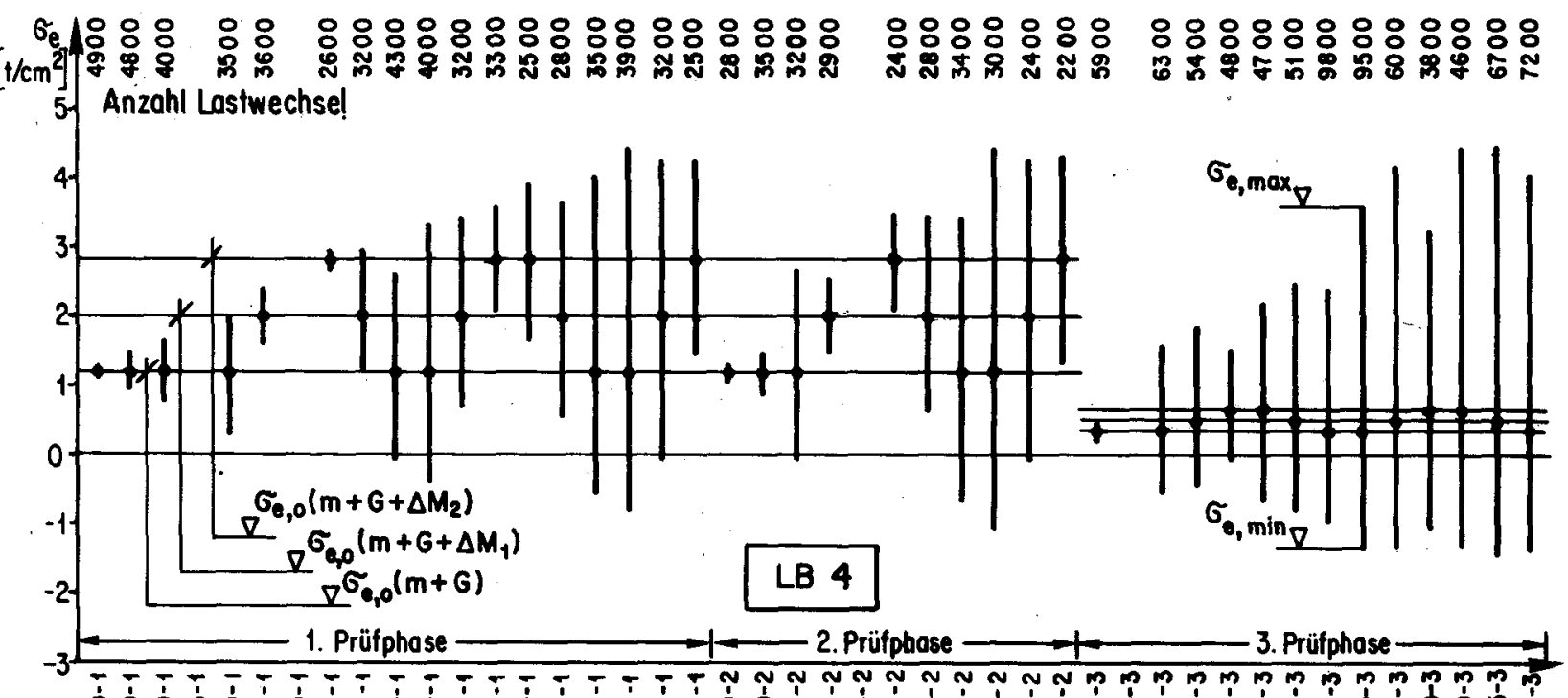

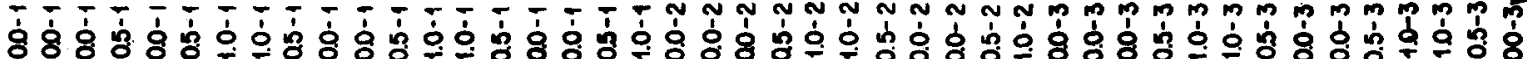

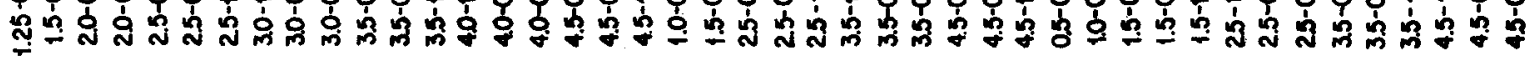

Bild 20b: Beanspruchung des Armierungsstahles, max. Schwingweiten und Lastwechsel je Beanspruchungsstufe des Bolkens LB 4 
$\left.\begin{array}{r}\left.\sigma_{\mathrm{e}}\right] \\ {\left[\mathrm{t} / \mathrm{cm}^{2}\right]} \\ 5\end{array}\right] \quad$ 品 Anzahl Lostwechsel

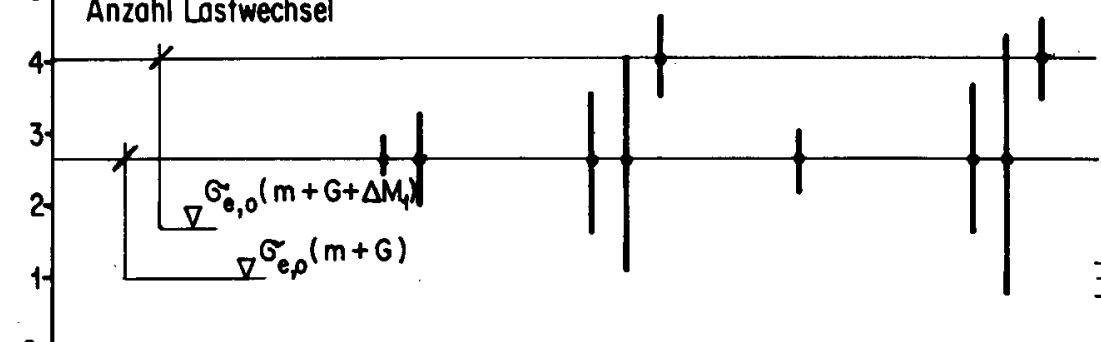

B 1
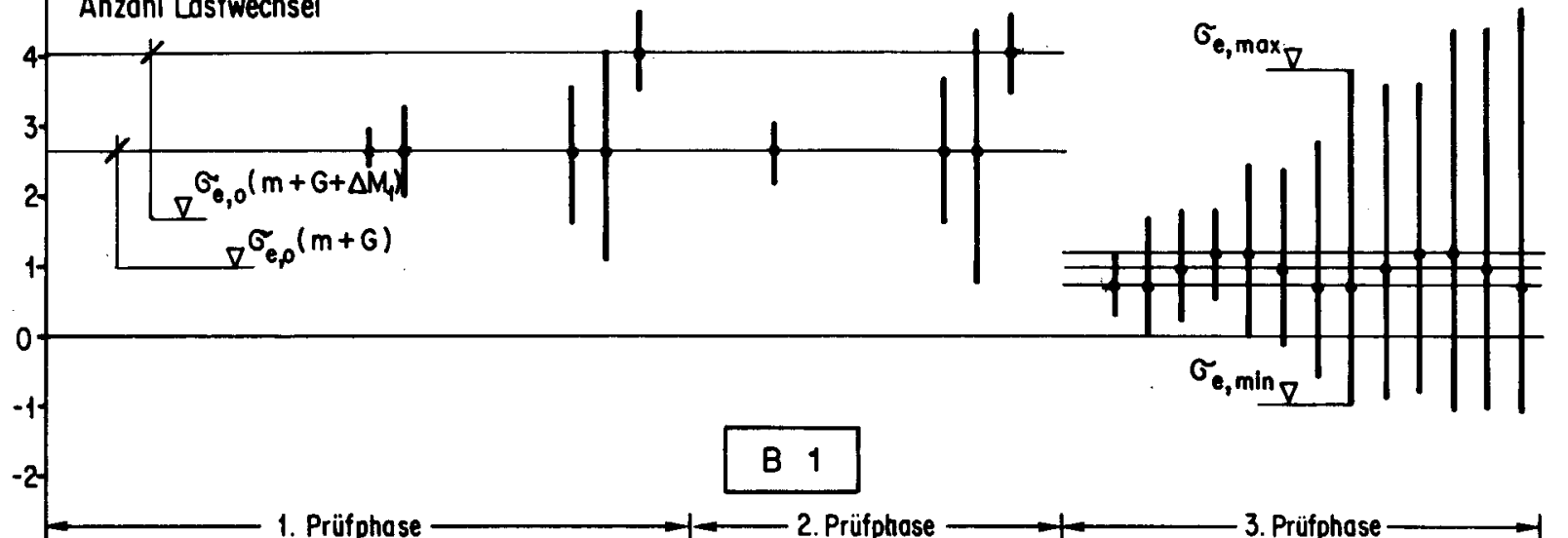

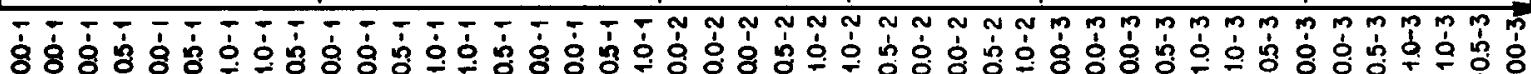

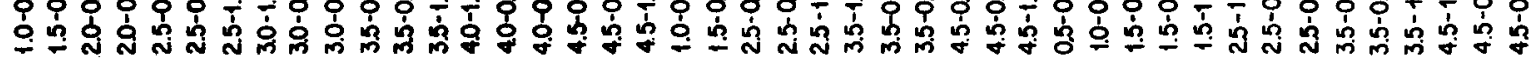

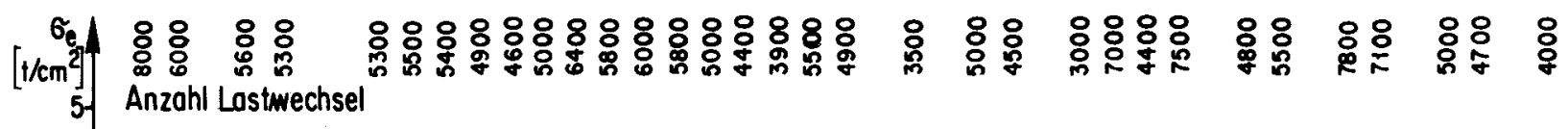

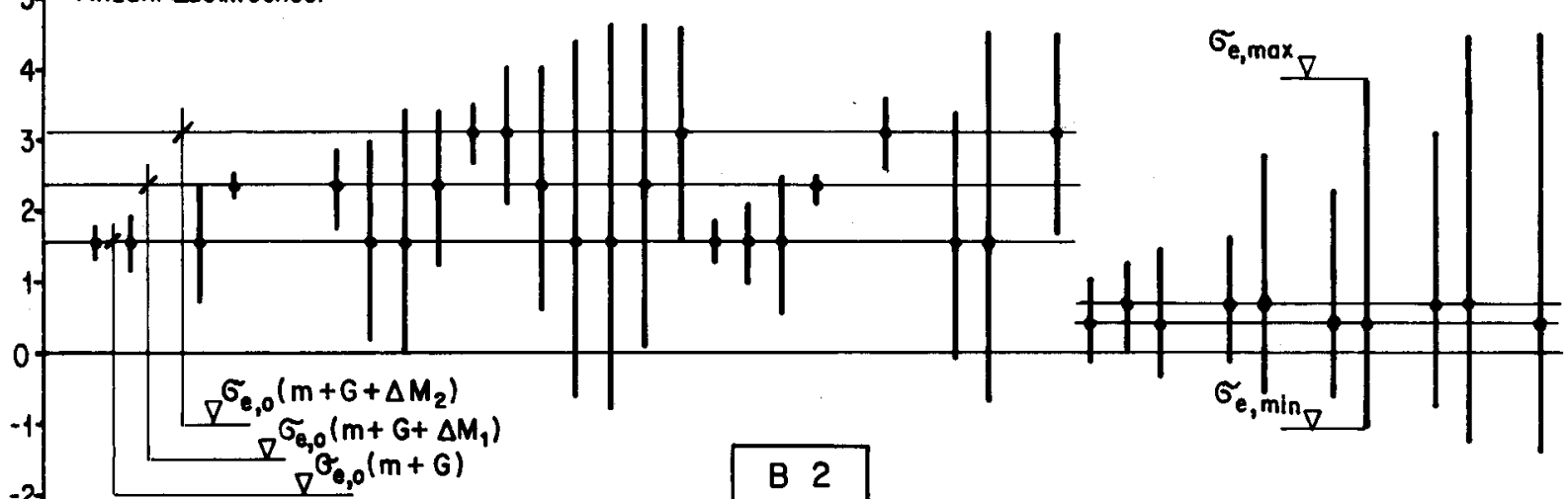

1. Prüfphose

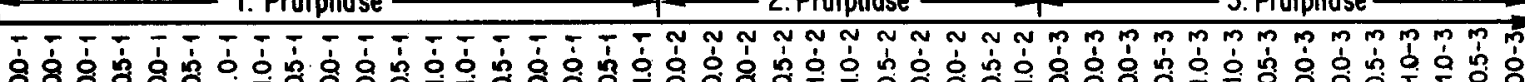

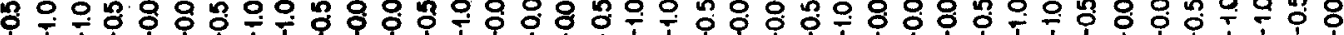

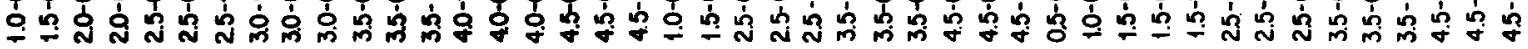

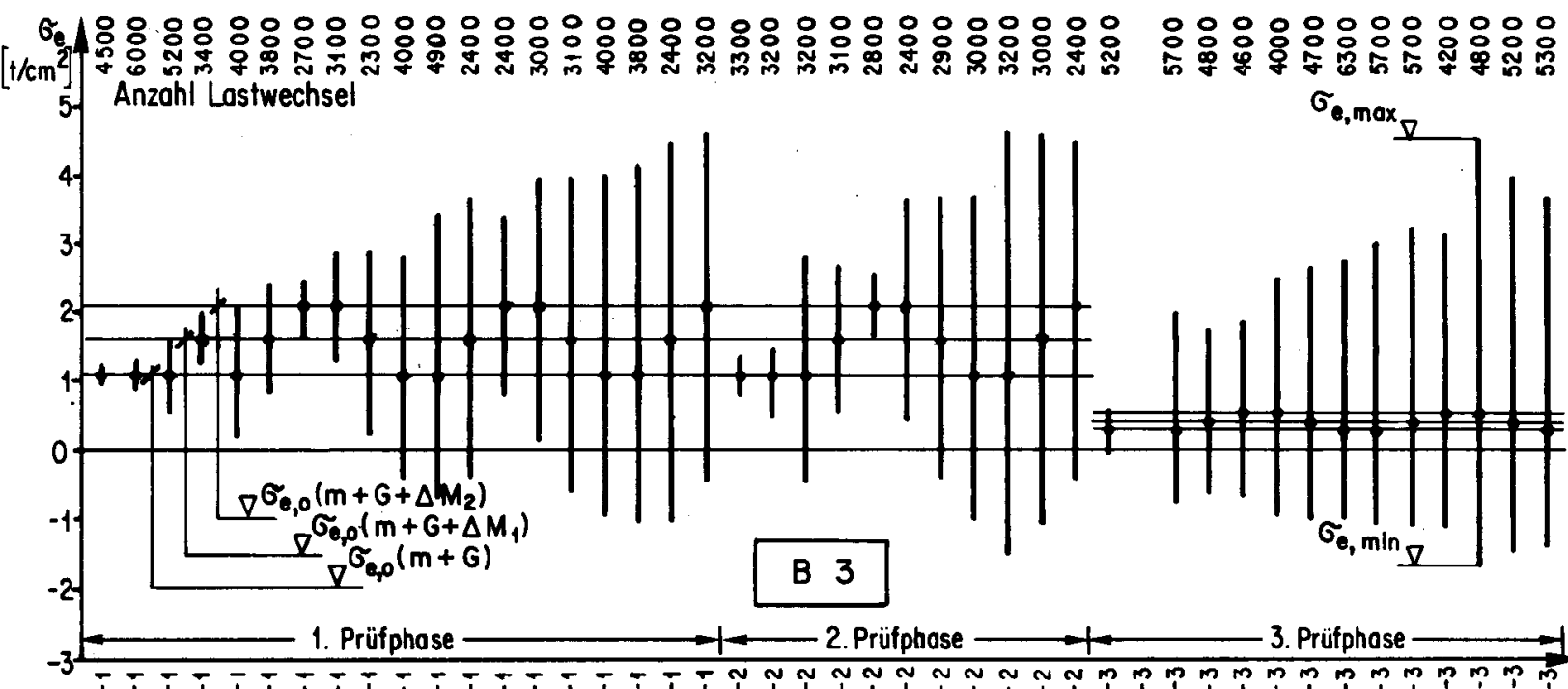



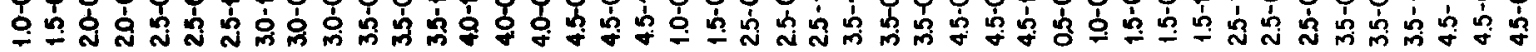

Bild 20c: Beanspruchung des Armierungsstahles, max. Schwingweiten und Lostwechsel je Beanspruchungsstufe der Balken B 1, B2, und B 3 

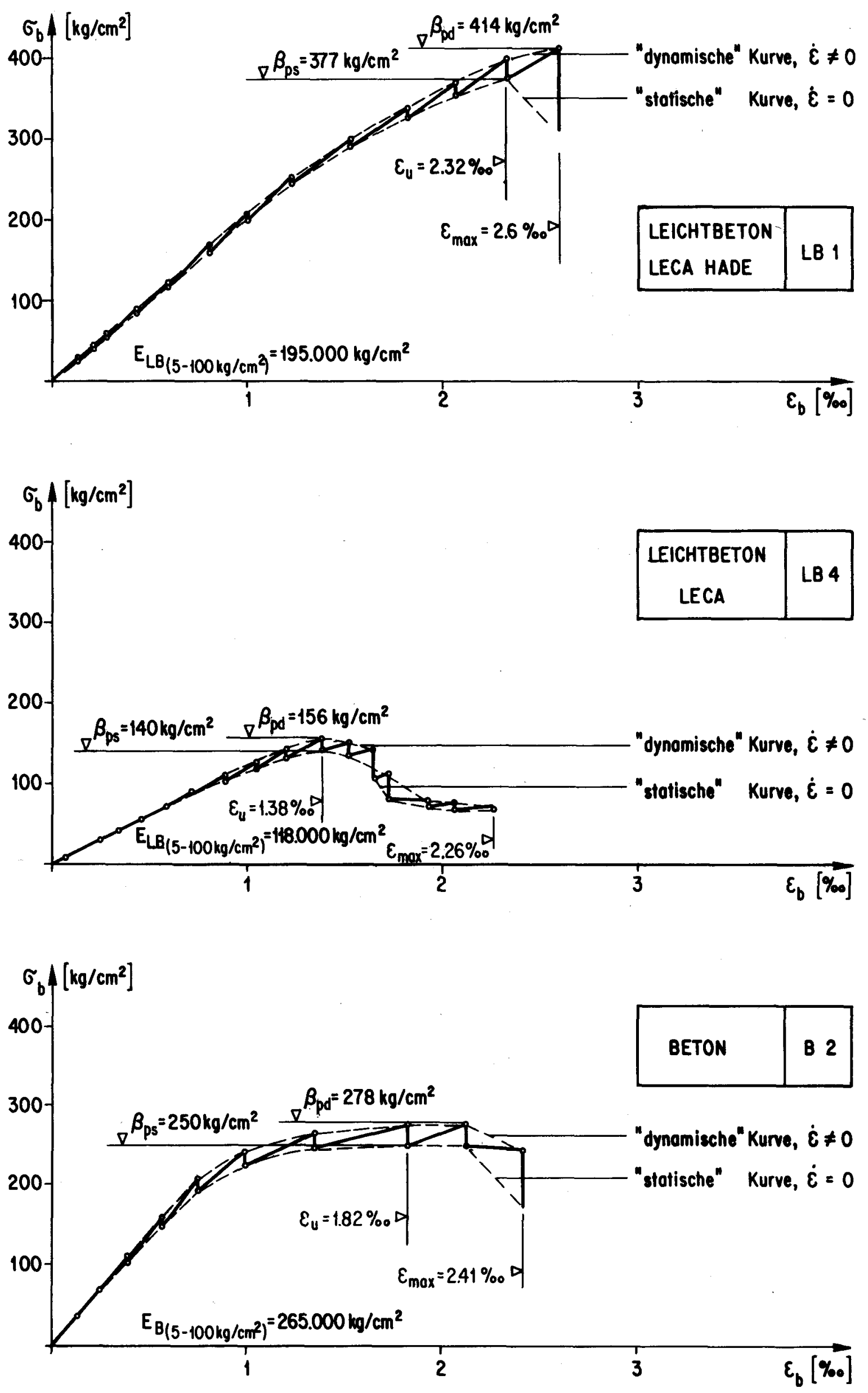

Bild 21: Spannungs-Dehnungs-Diagramme einzelner Leichtbeton-/Beton-Prismen 


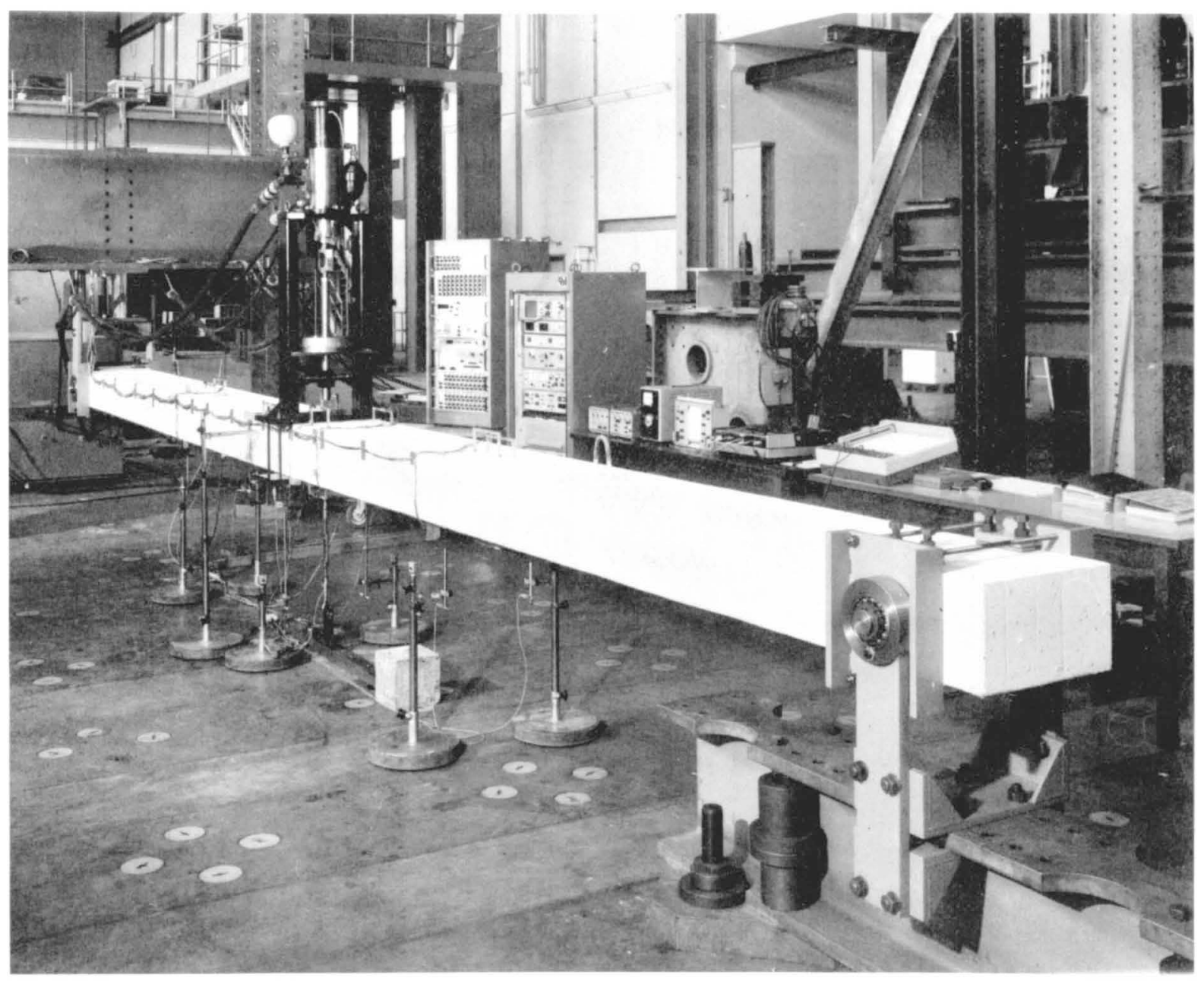

Bild 22: Versuchsanlage

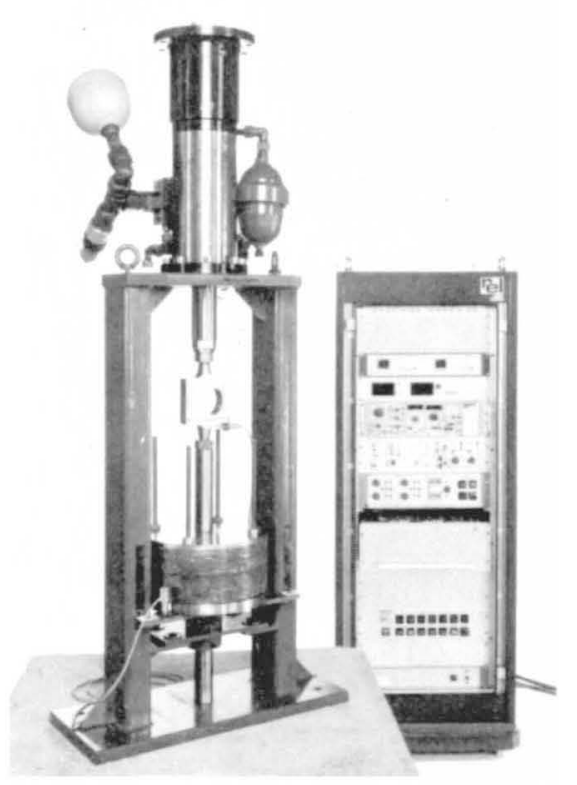

Bild 24: Schwingungserreger 

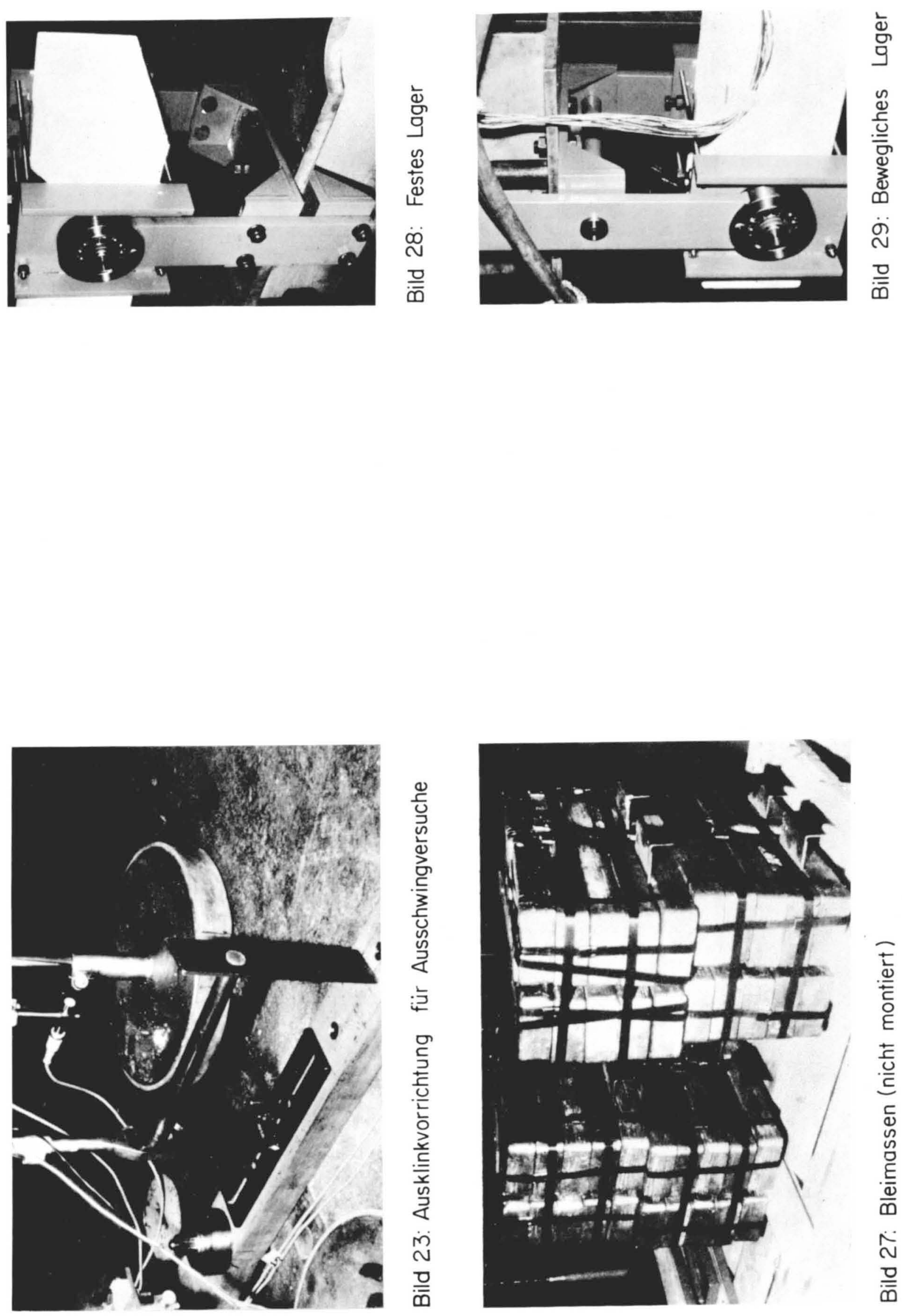


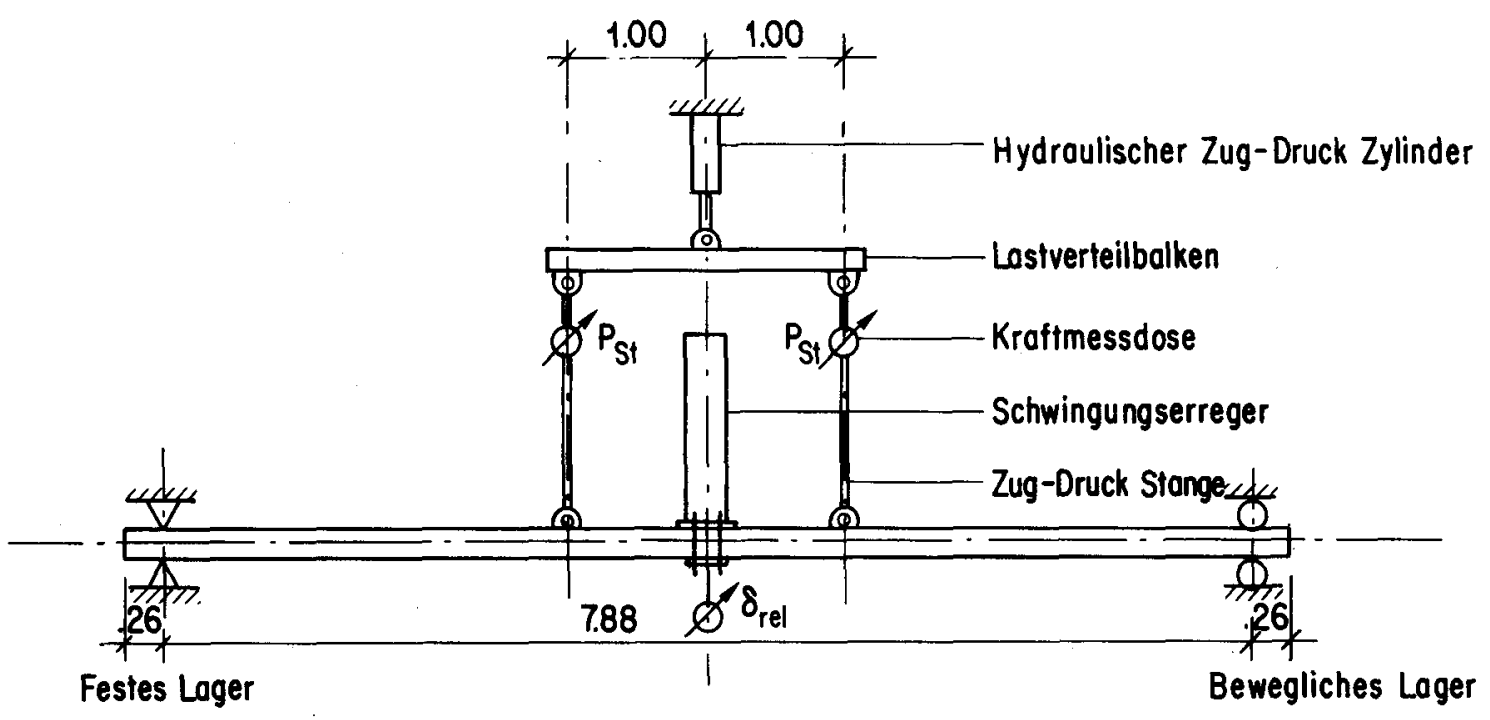

Bild 25: Statische Belastungsvorrichtung, schematische Darstellung

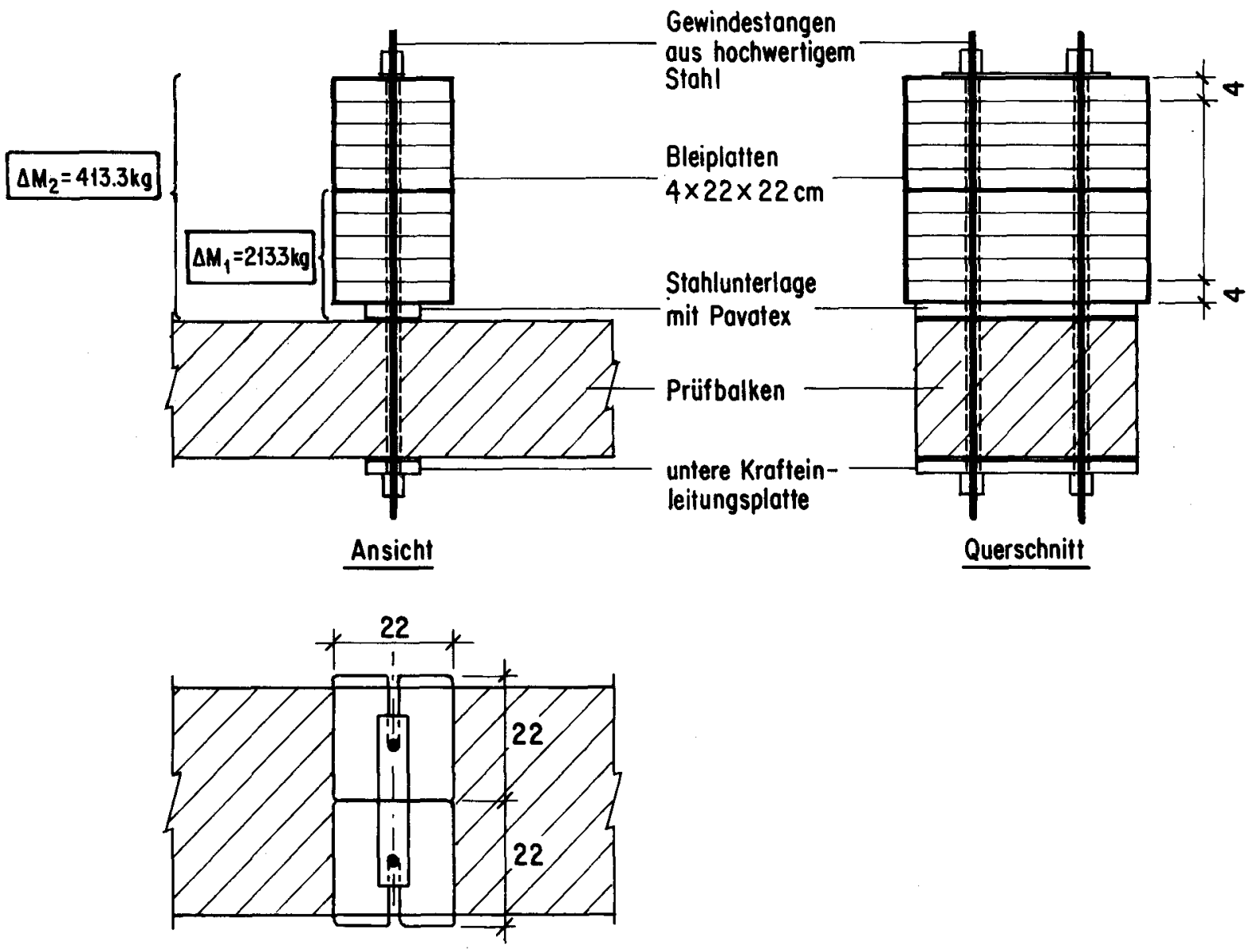

Grundriss

Bild 26: Lage und Anordnung der Zusatzmassen $\left(\Delta M_{1}, \Delta M_{2}\right)$ auf den Prüfbalken, Vergl. auch Bild 17 

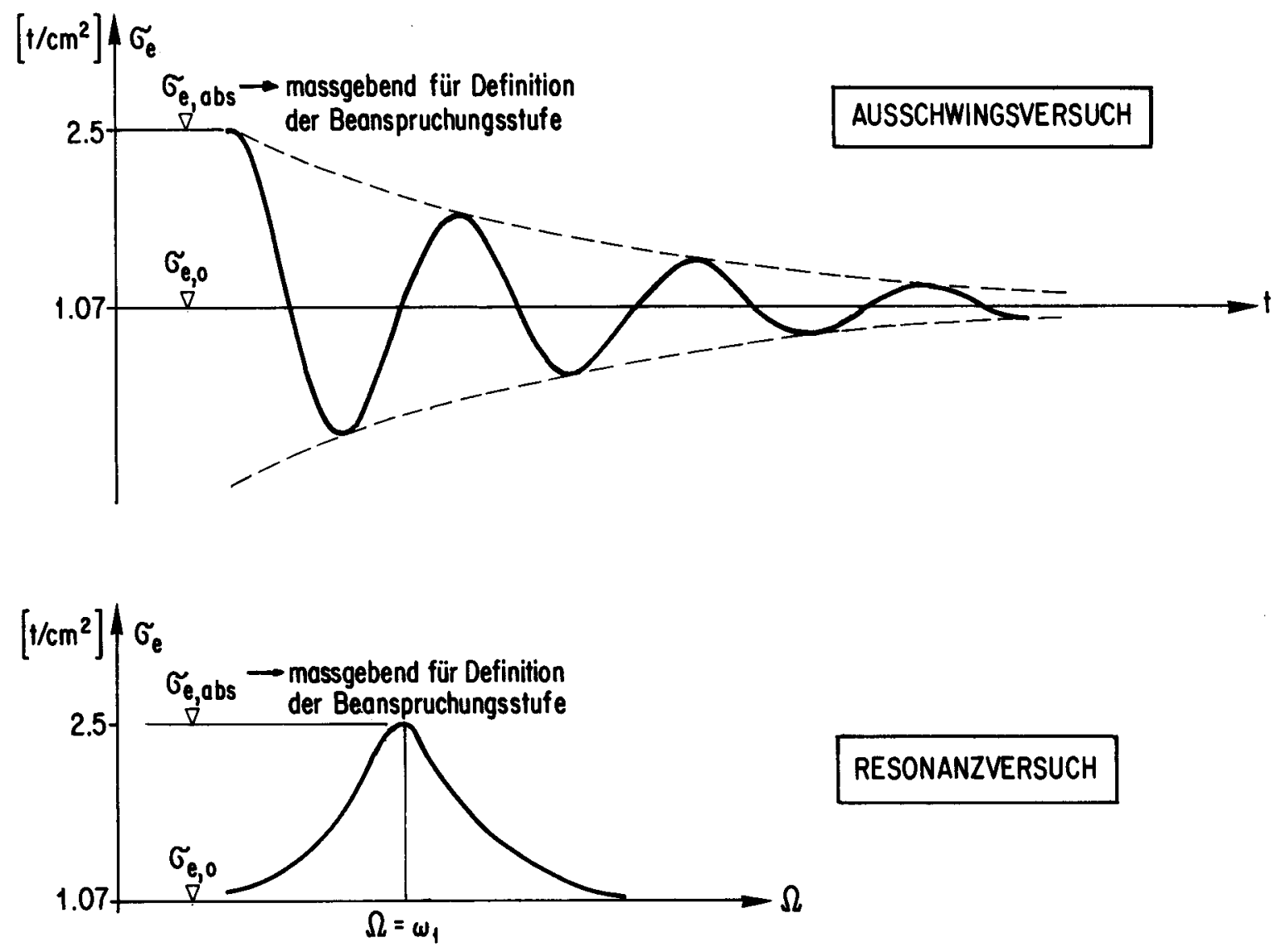

RESONANZVERSUCH

Bild 30: Definition der Beanspruchungsstufen (Balken B3, Beanspruchungsstufe 2.5-0.0-1)

$A=$ Ausschwingen $\quad R=$ Resononzkurve

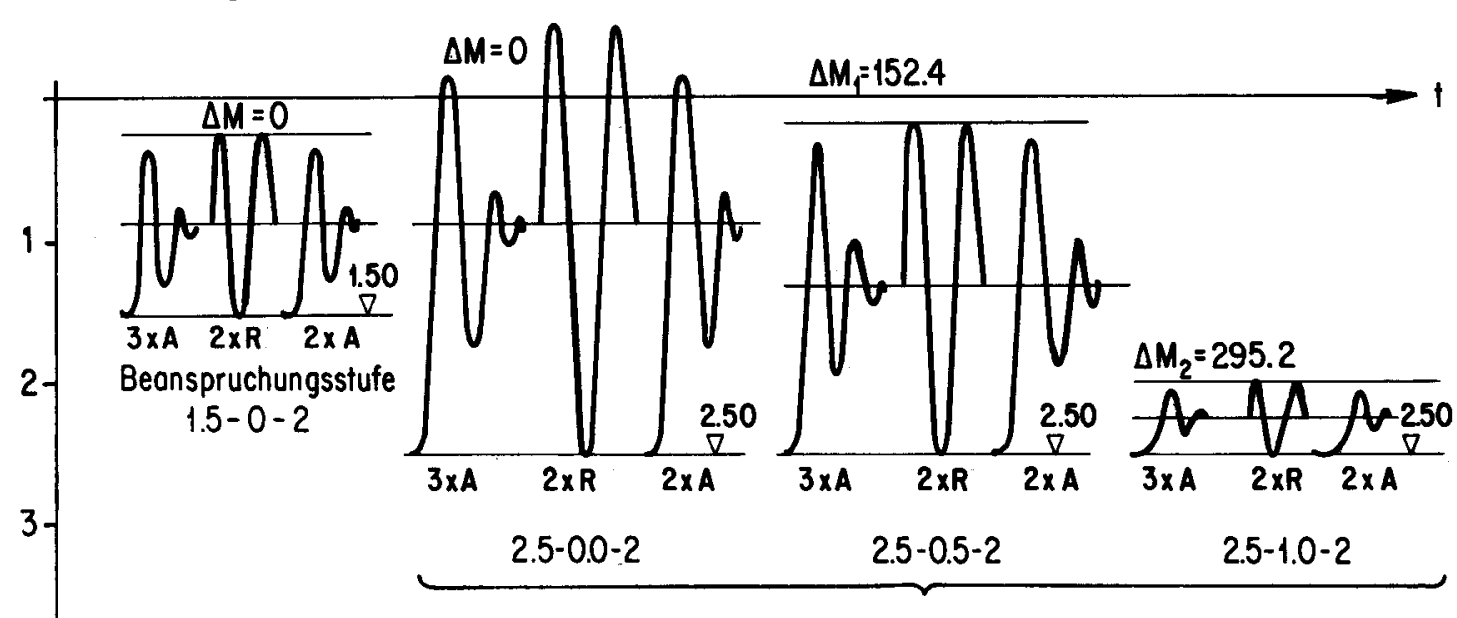

Beanspruchungsstufen 2.5 -..-2

$5-4$

Bild 31: Ausschnitt aus dem Prüfprogramm des Balkens LB 3 

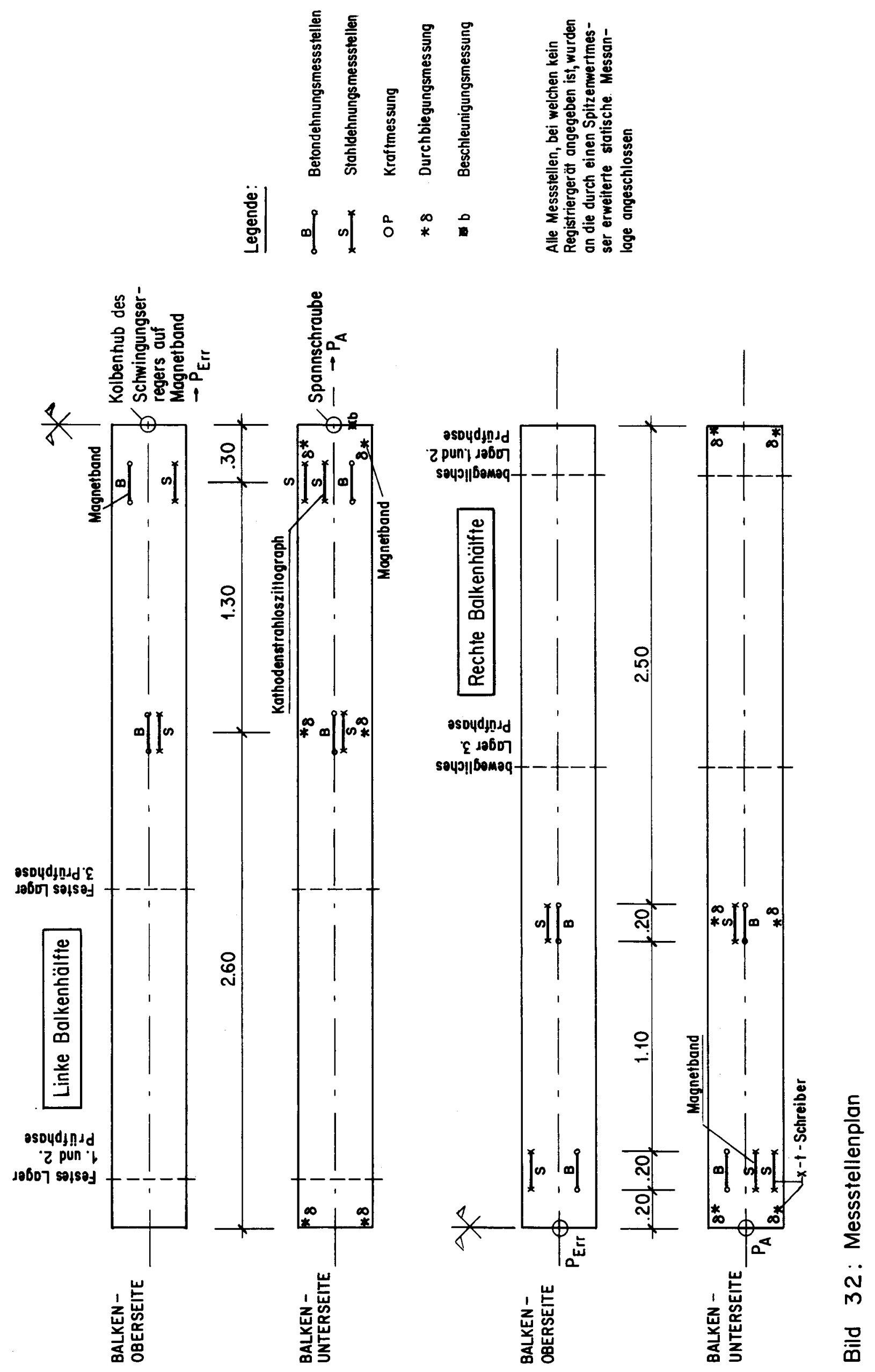

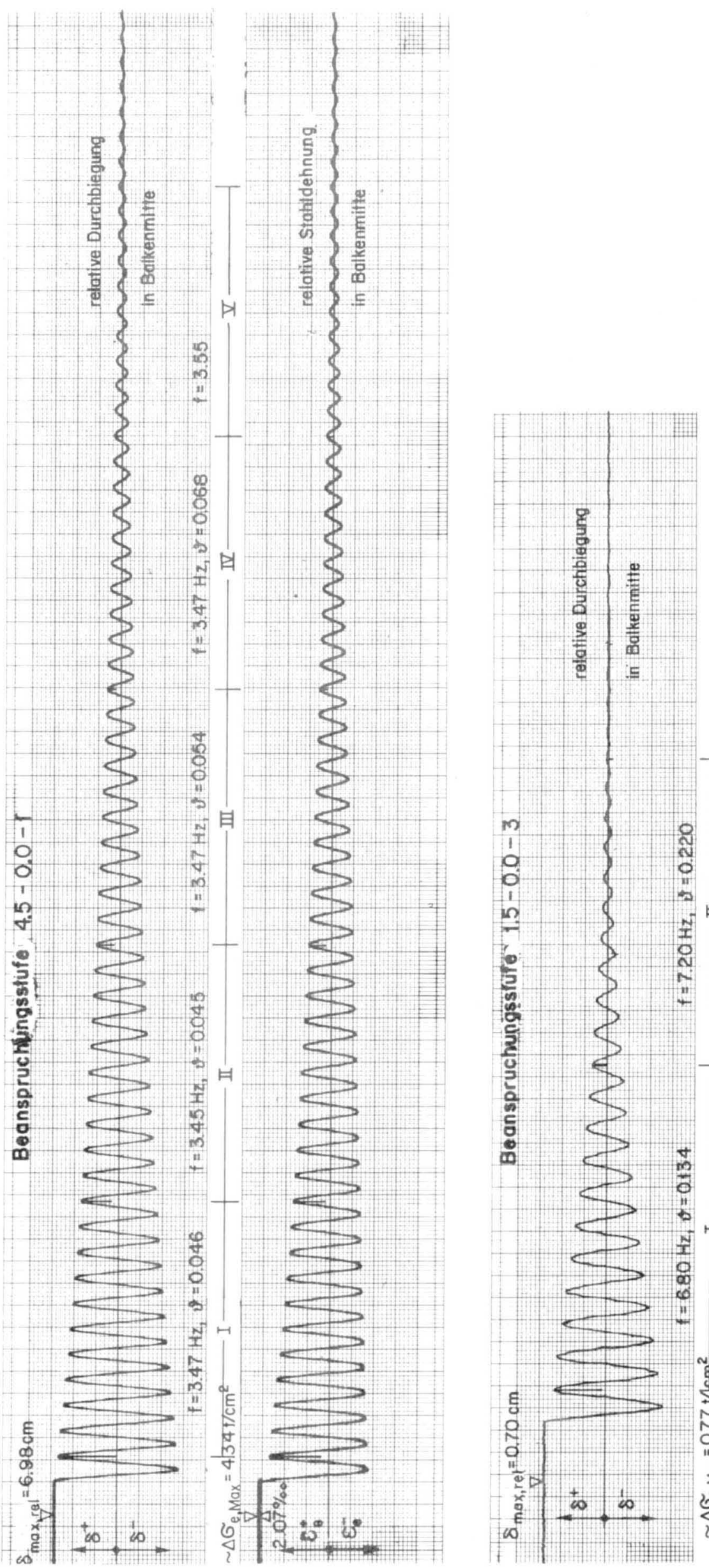

5

웅

เ̊

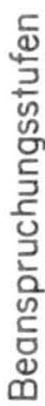

$m$

๑

동
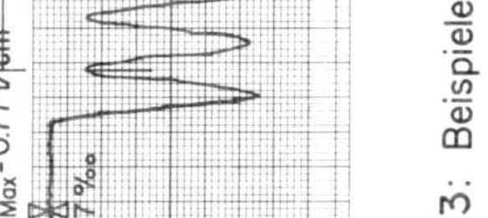

๓

$\ddot{m}$

흠 


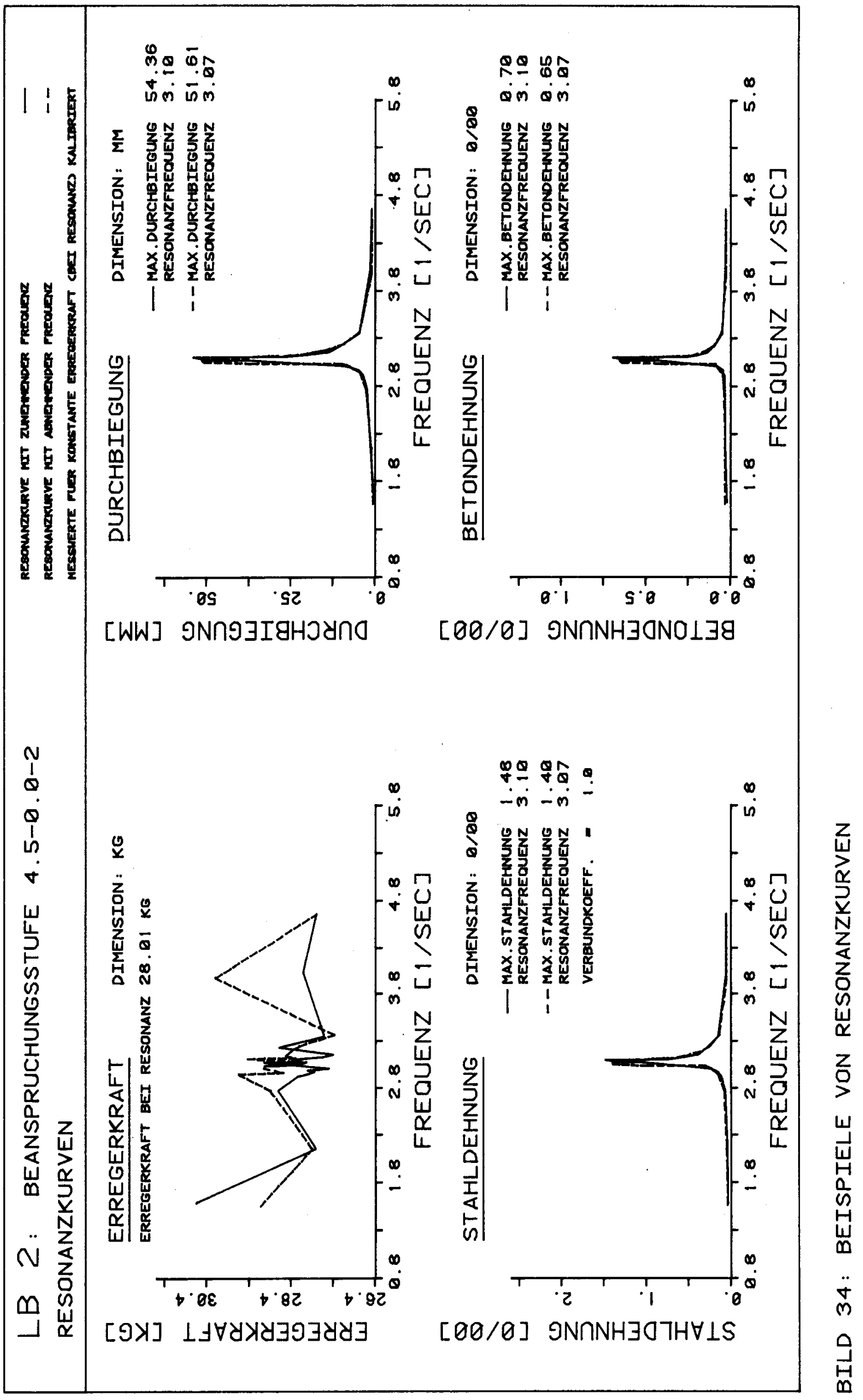



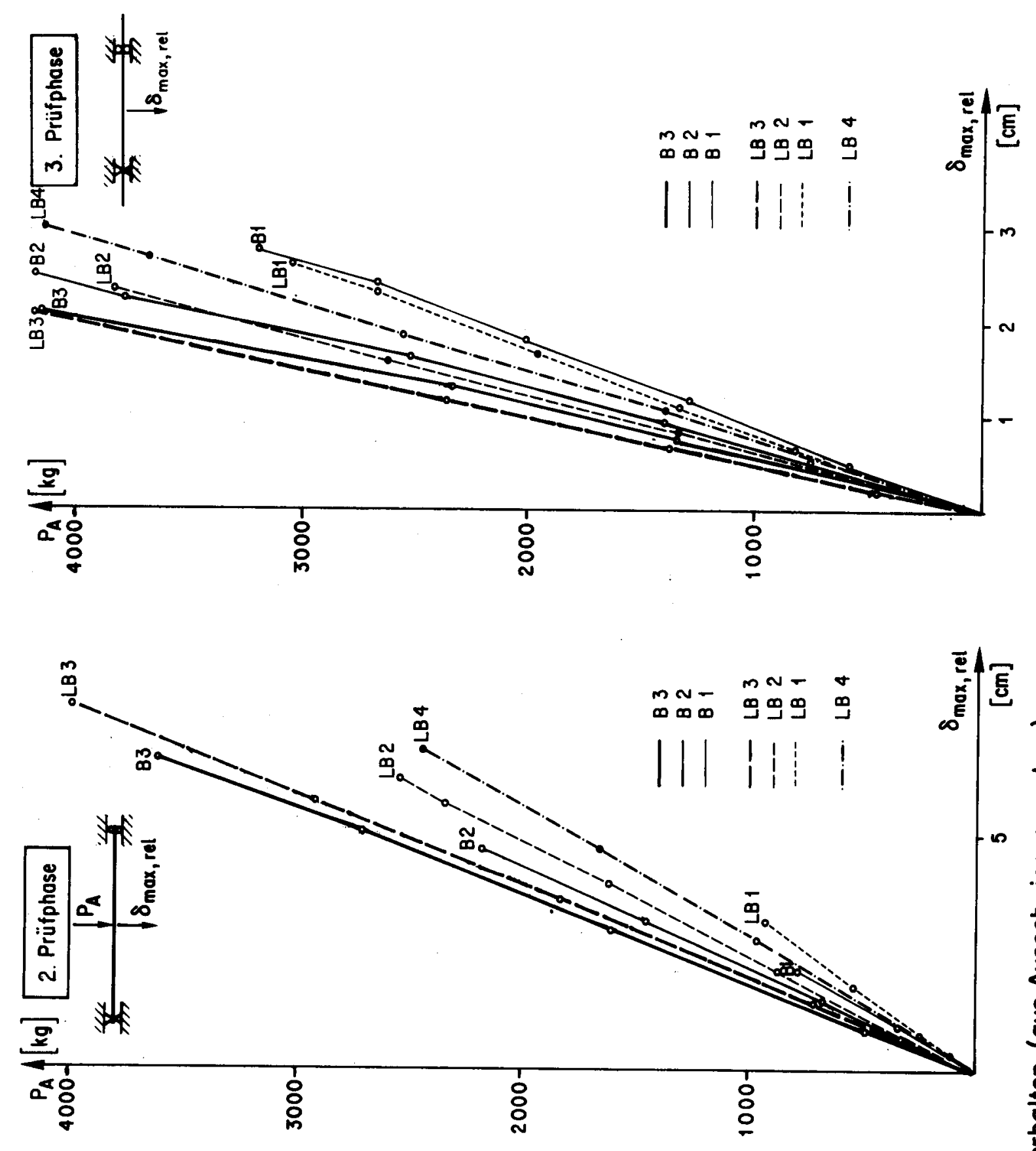

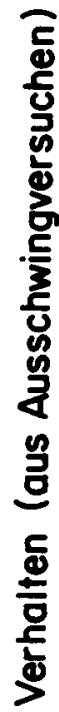

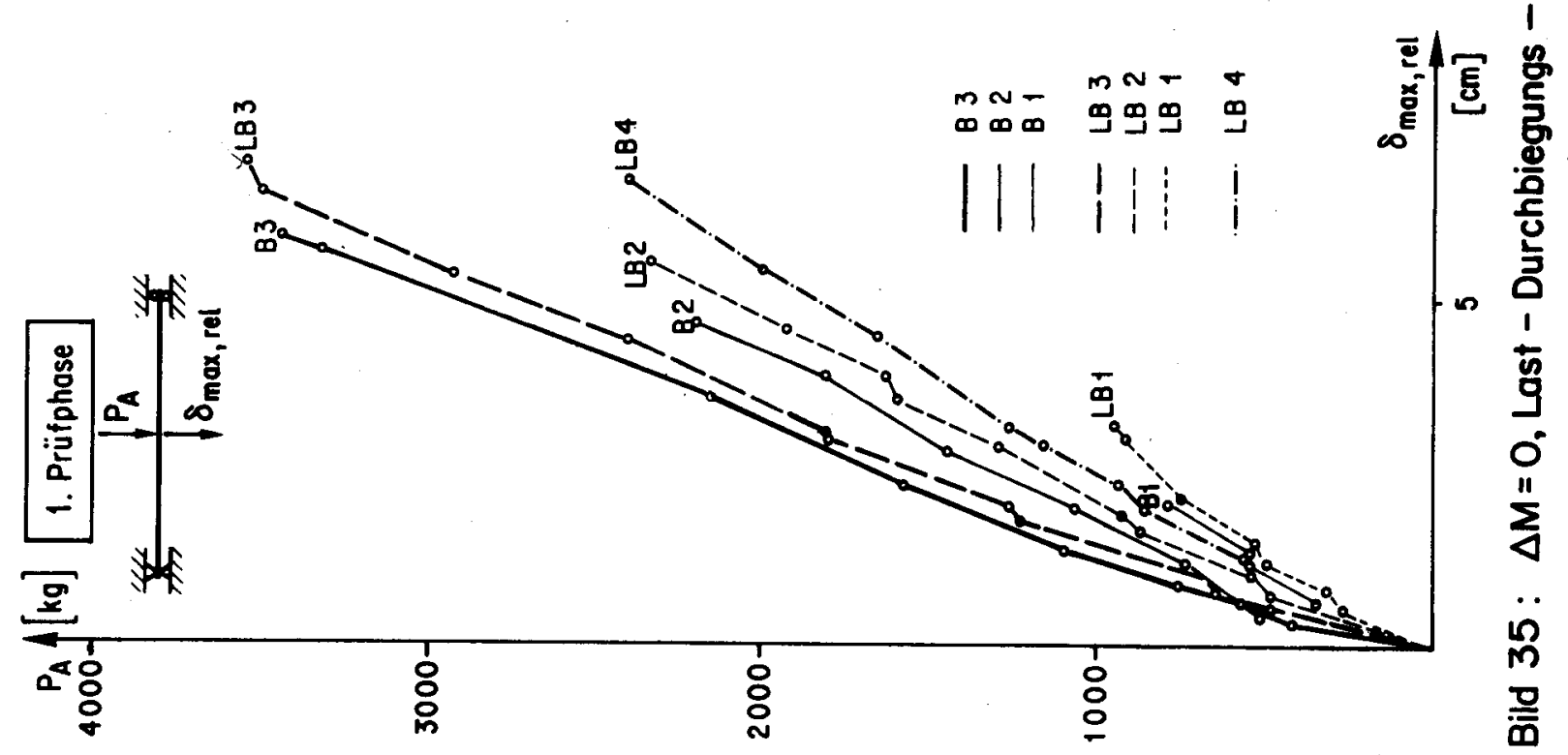



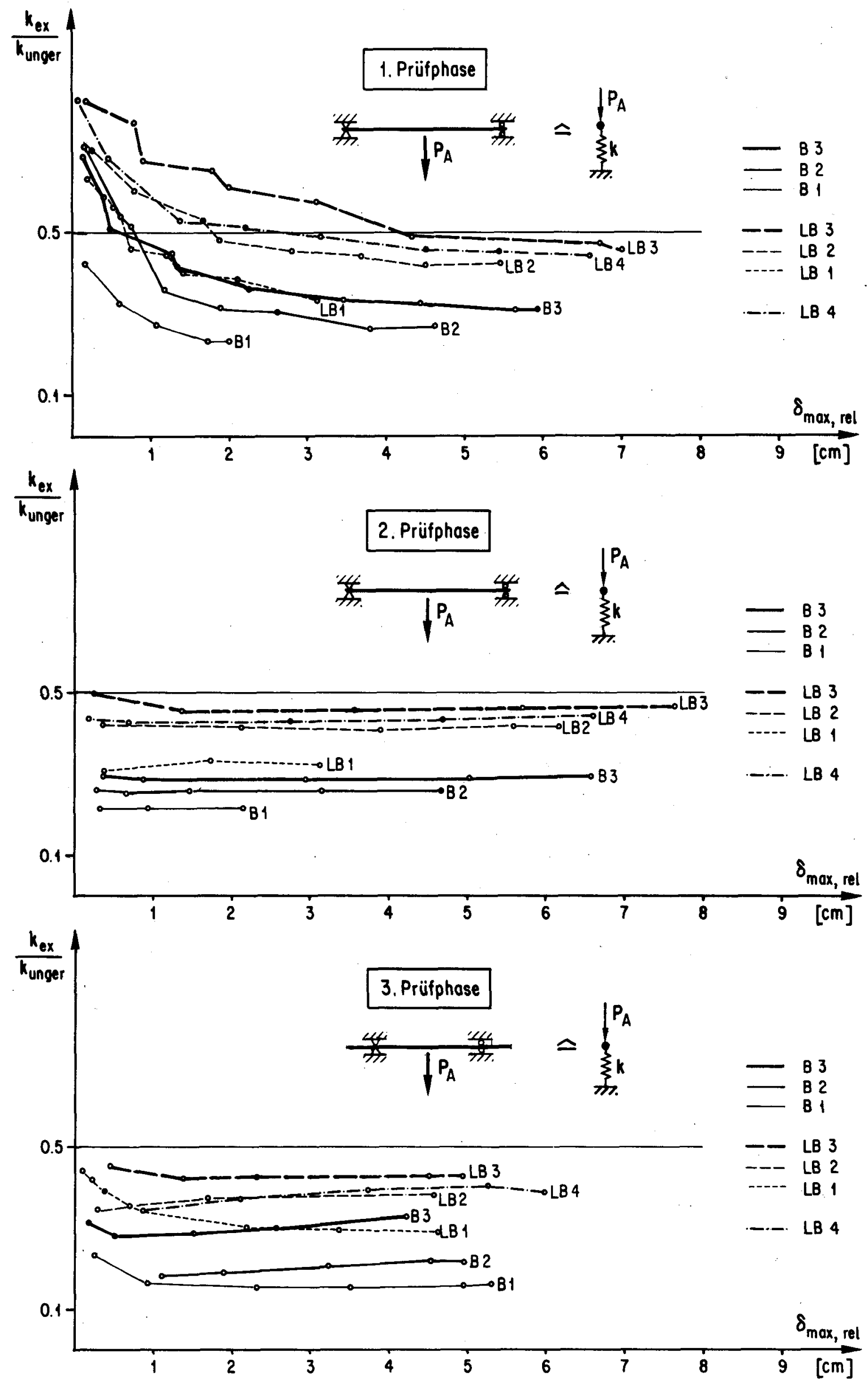

Bild 36: $\Delta M=0$, Verhältnis der gemessenen zur ungerissenen Sekantensteifigkeit (aus Ausschwingversuchen ) 


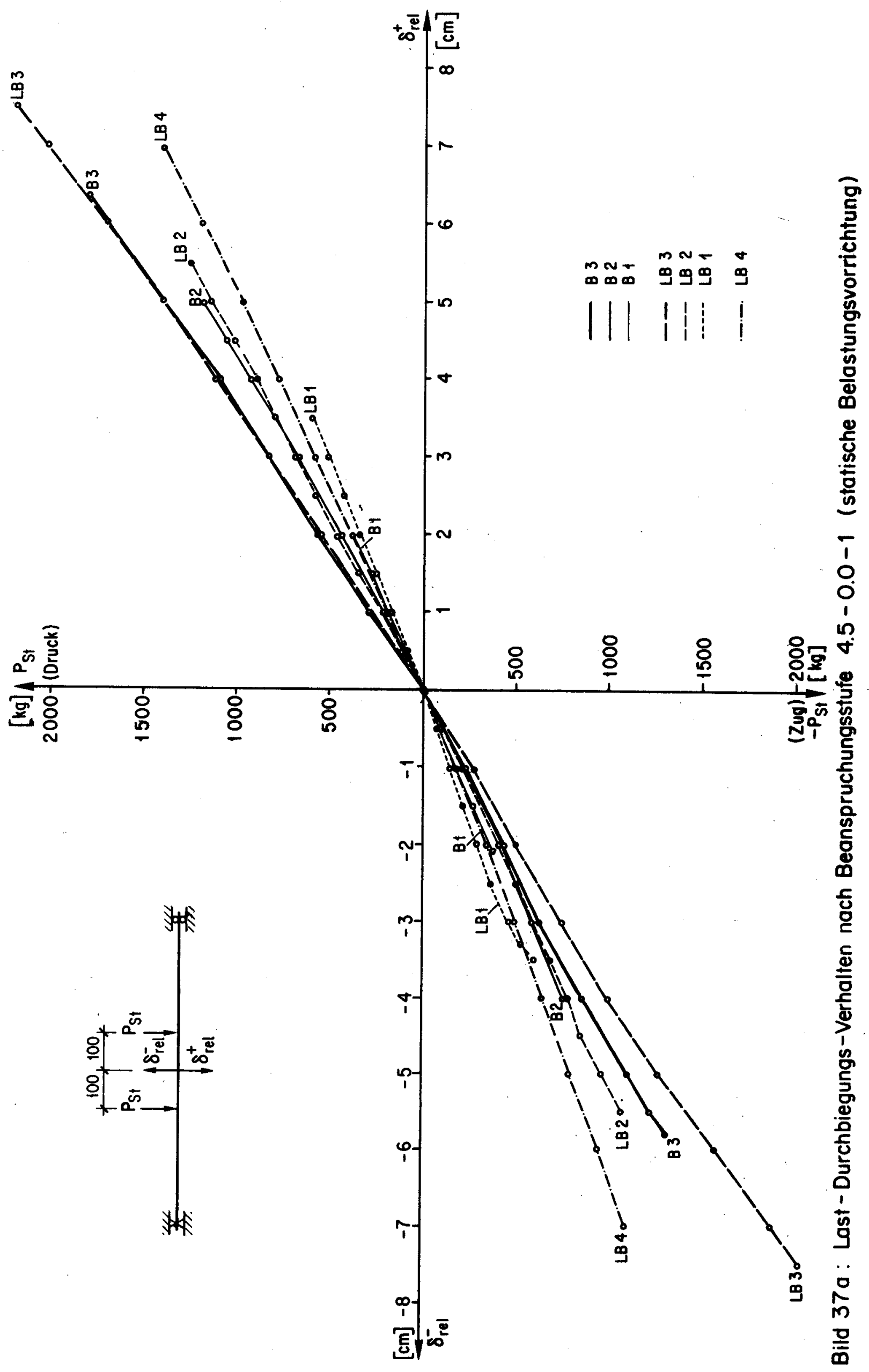




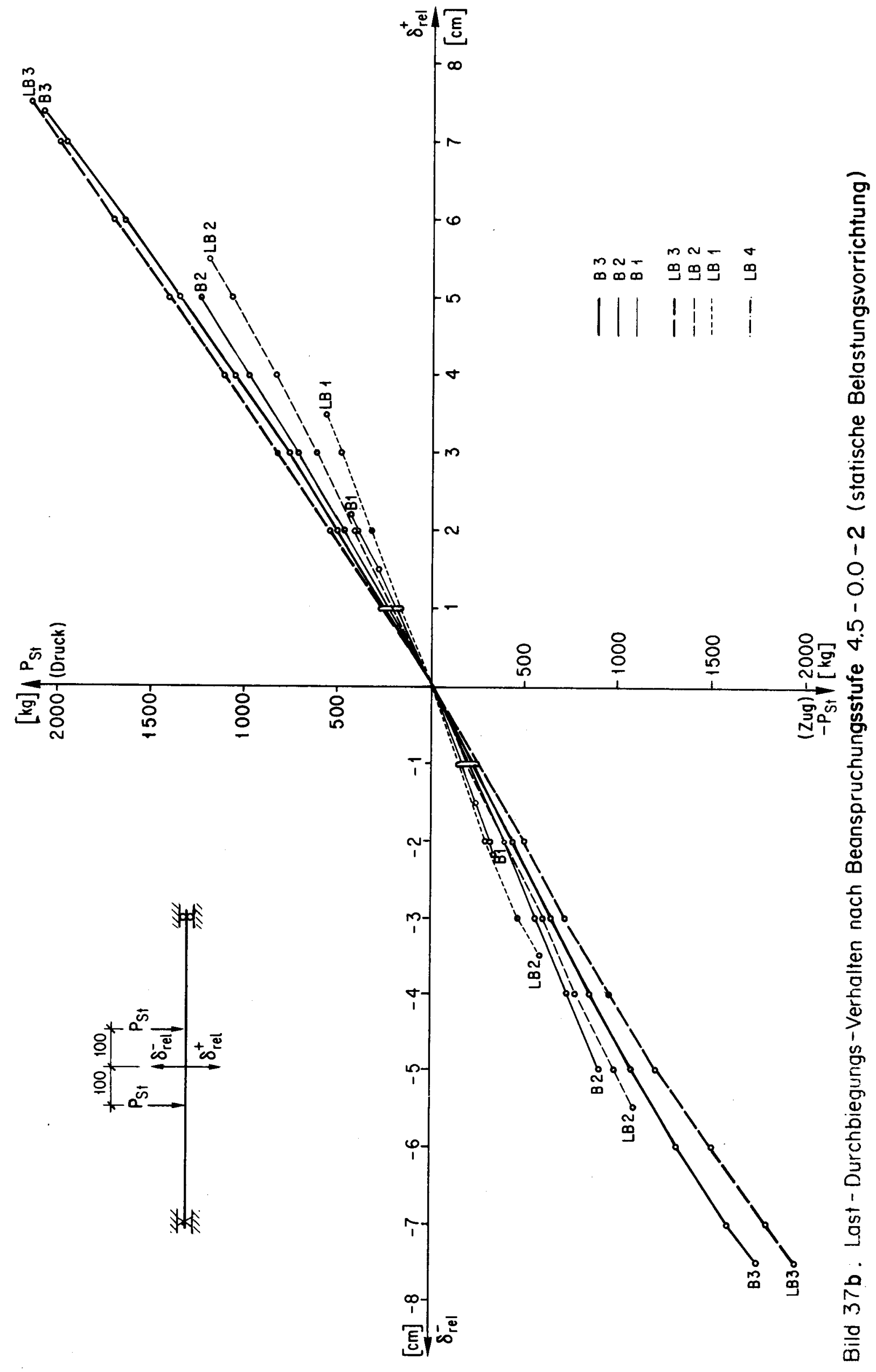



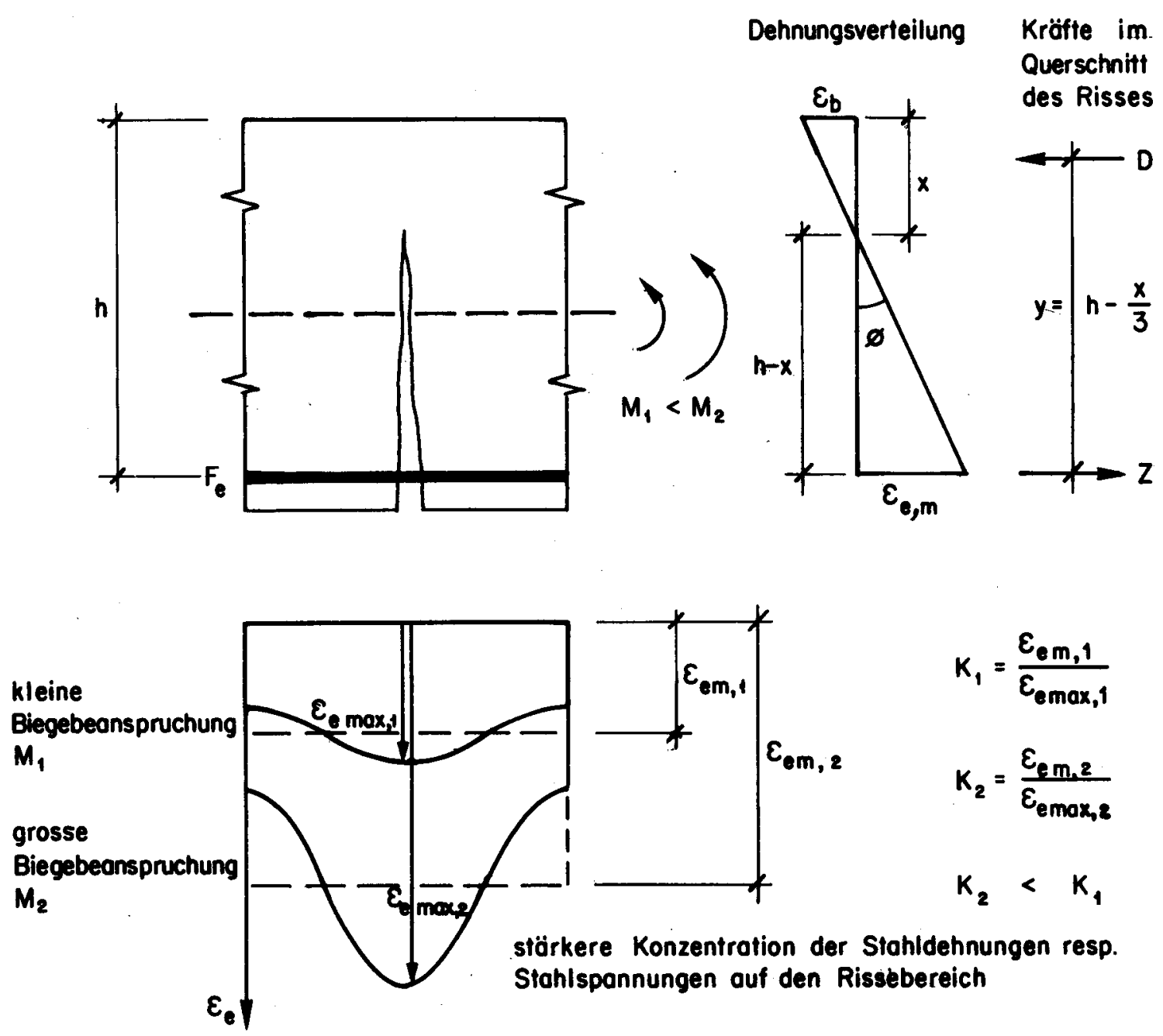

Bild 37c: Verlauf der Stahldehnungen in einem Biegerisselement 


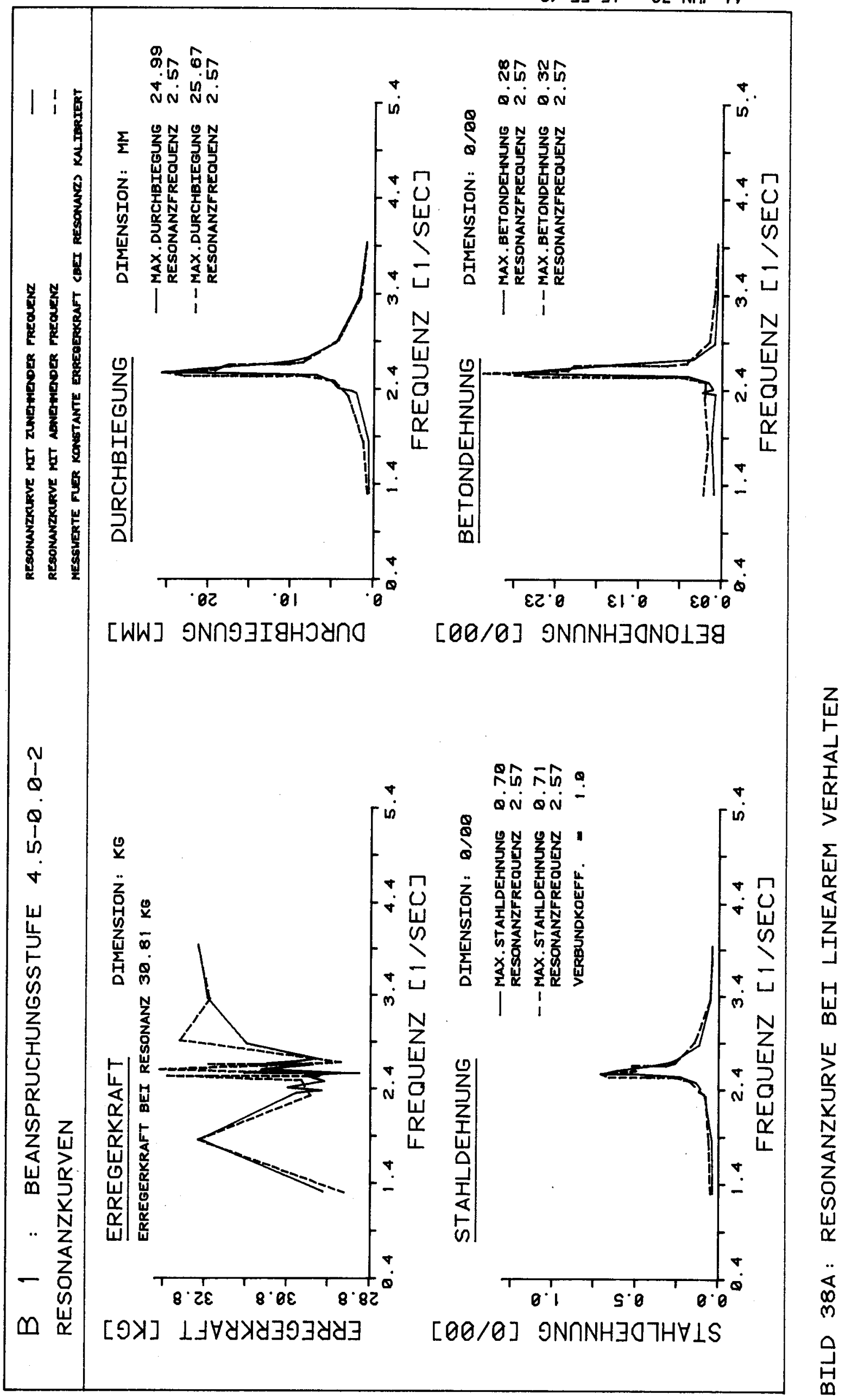




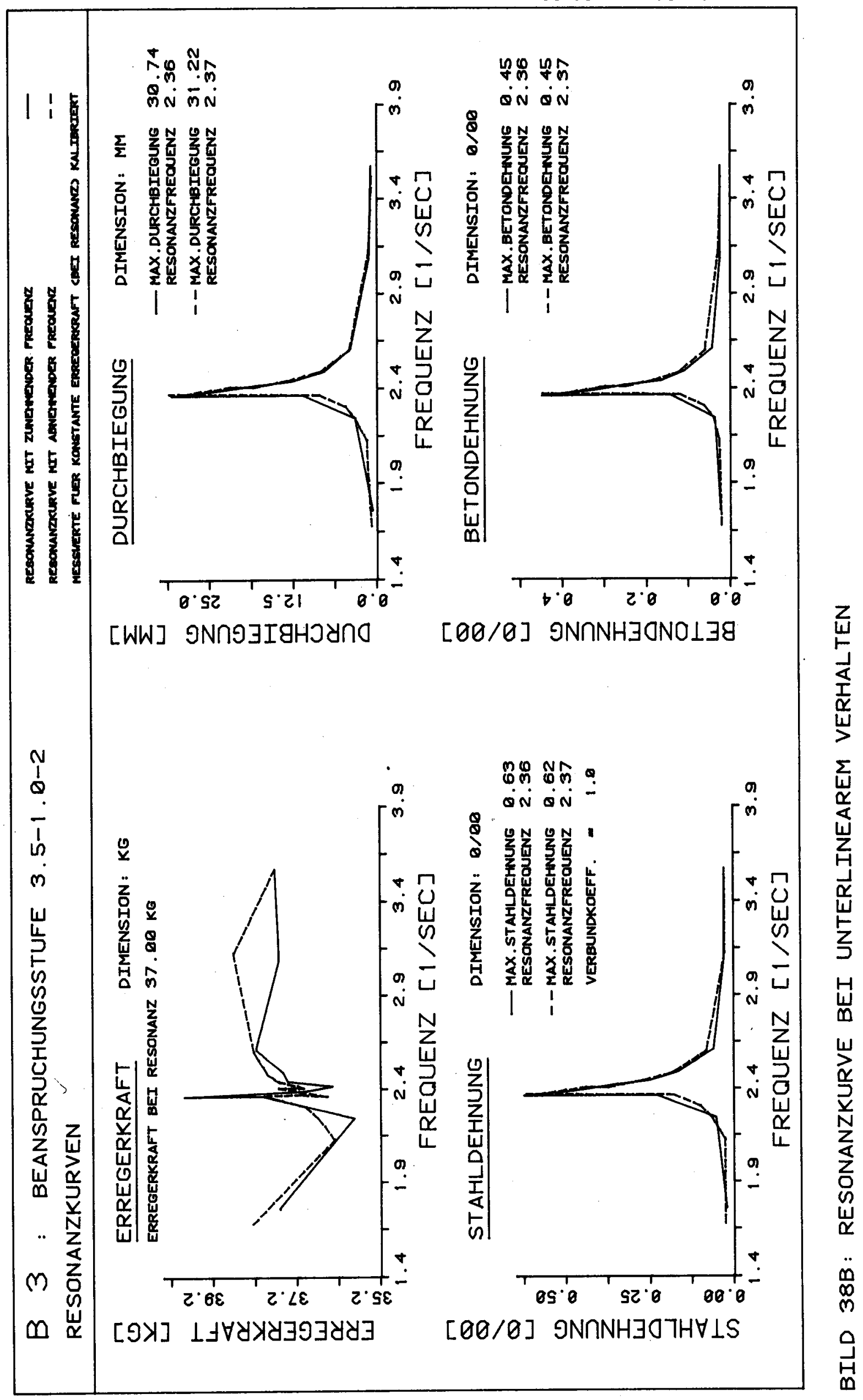




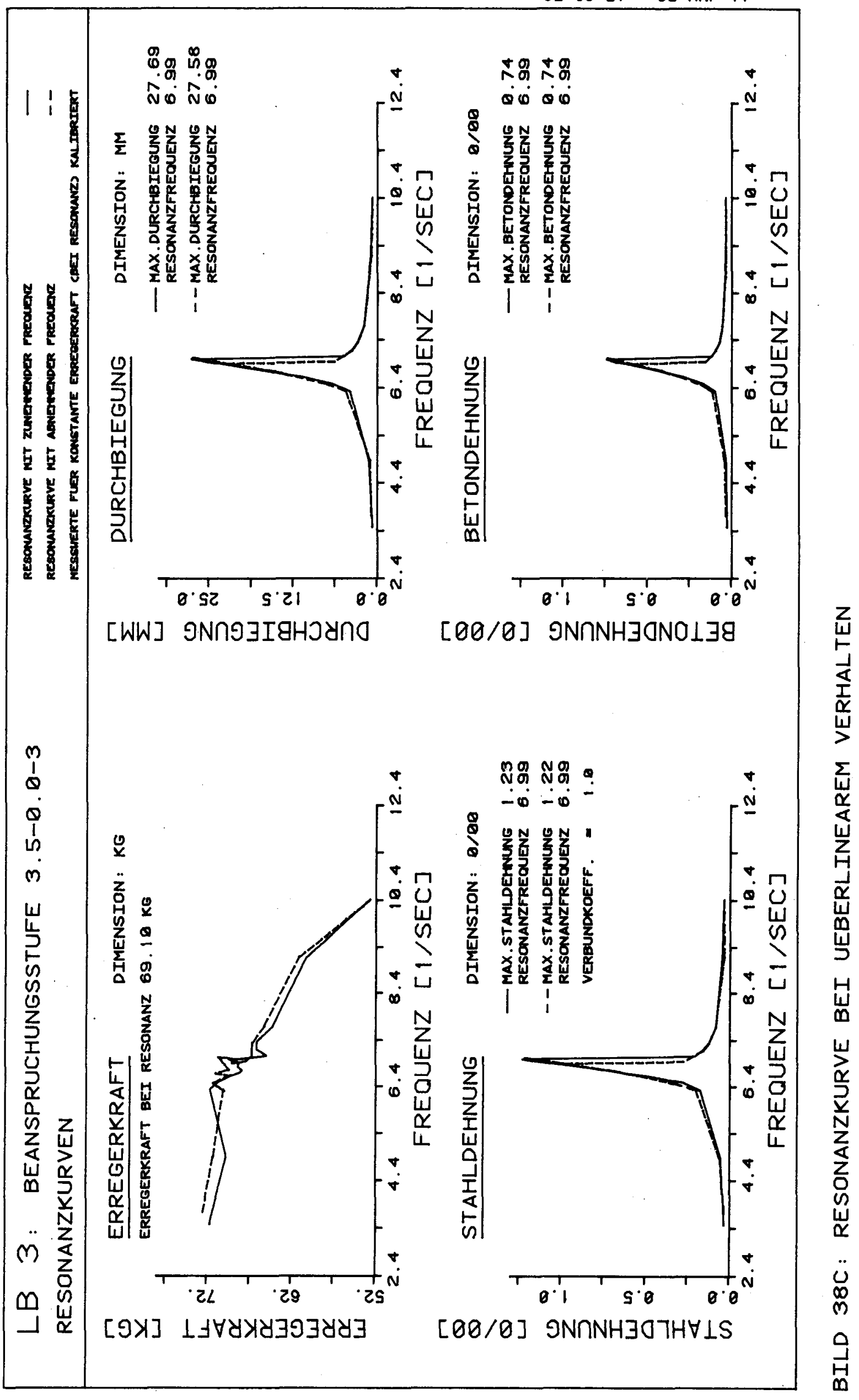



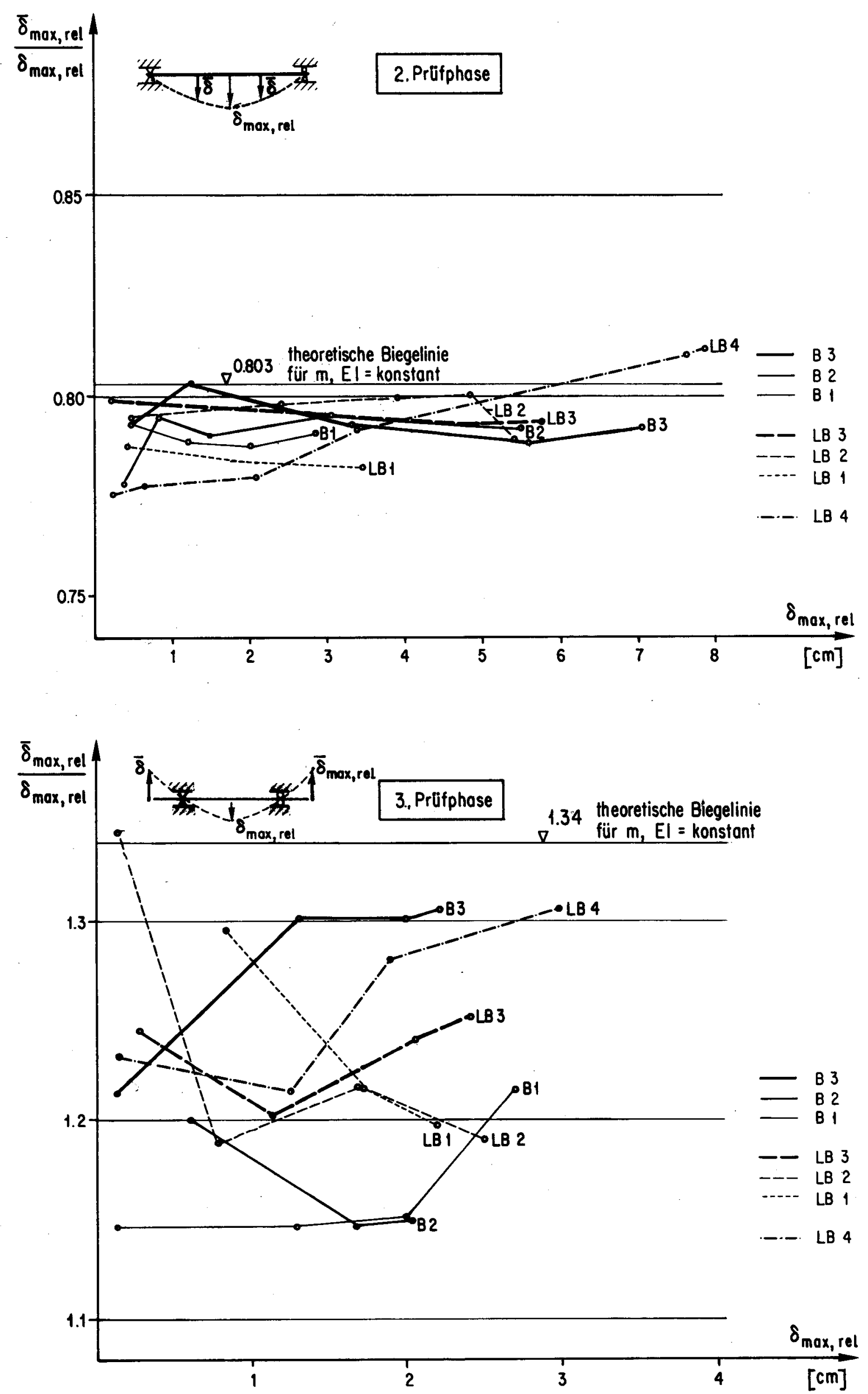

Bild 39: $\Delta M=0$, Verlouf der relativen Biegelinie in Funktion der maximalen relativen Durchbiegung $\delta_{\text {max,el }}$ 

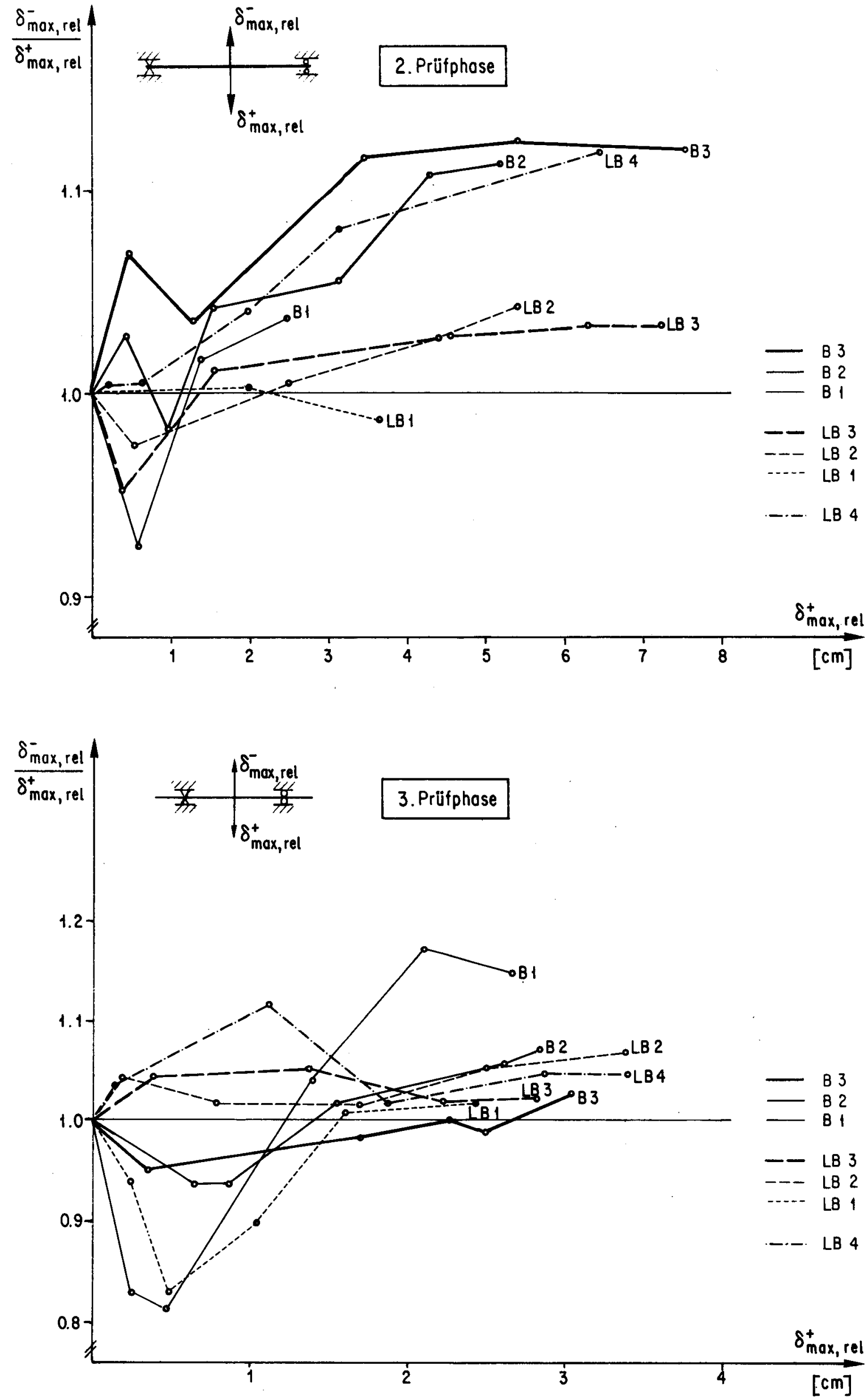

Bild 40: $\Delta M=0$, Verhältnis der maximalen negativen und positiven relativen Durchbiegungen $8_{\max }$, rel 


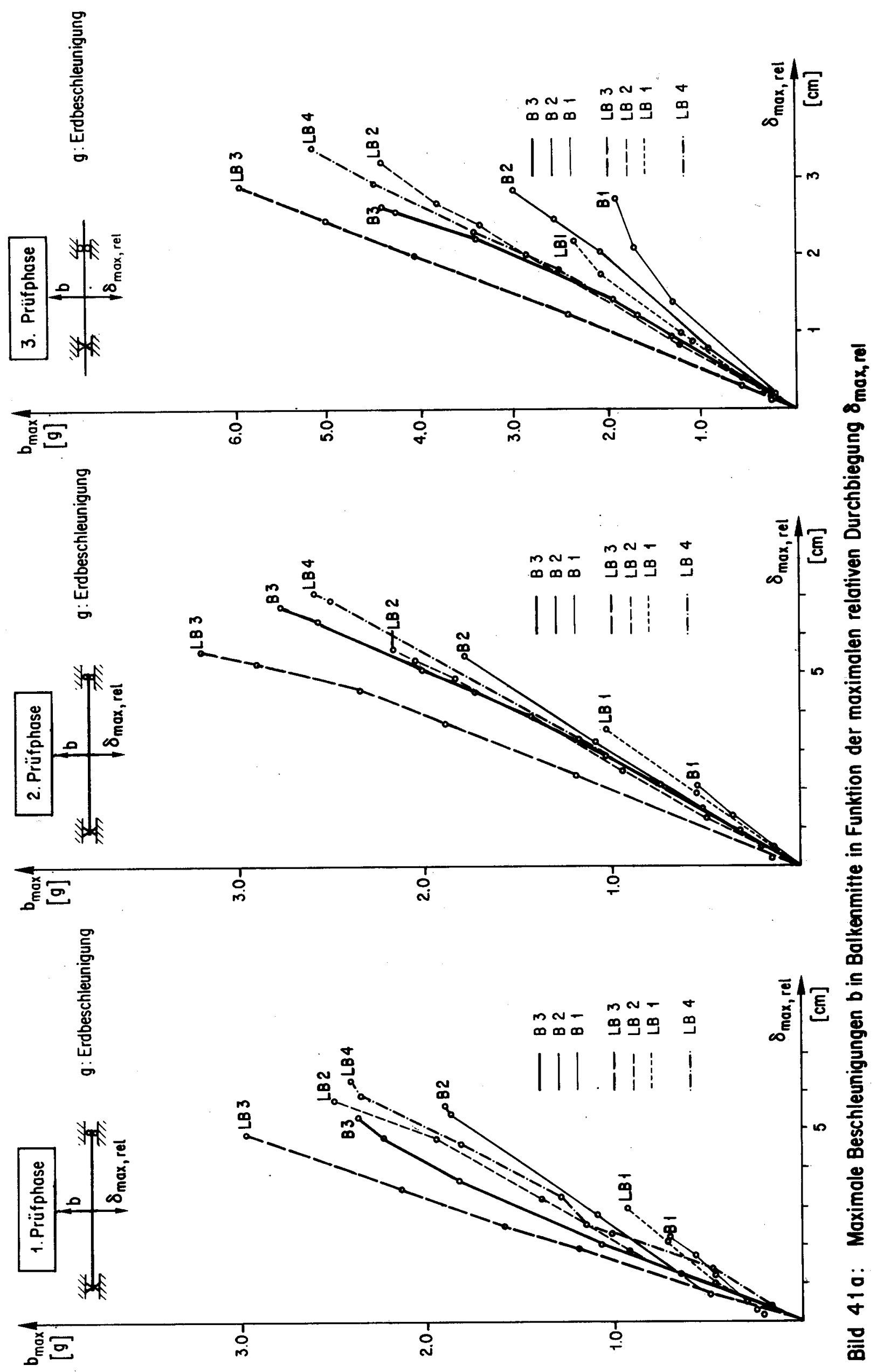




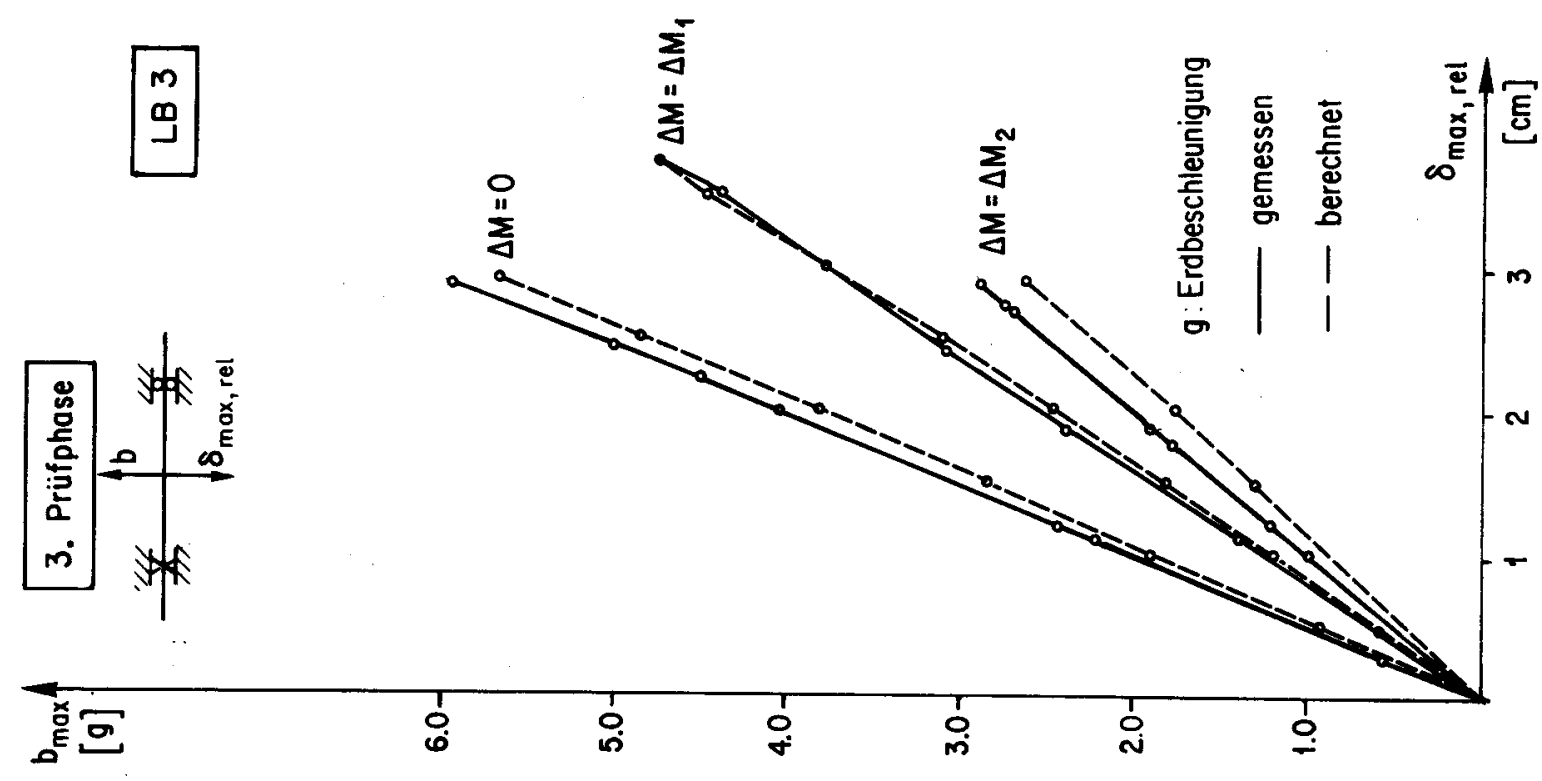

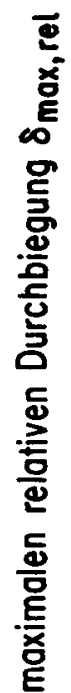
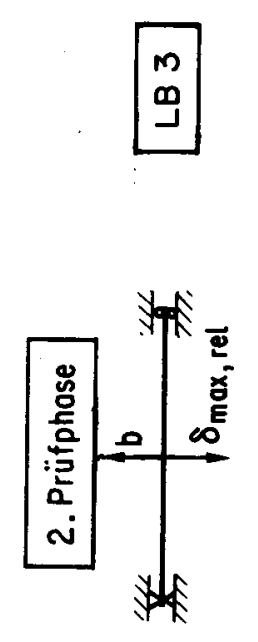

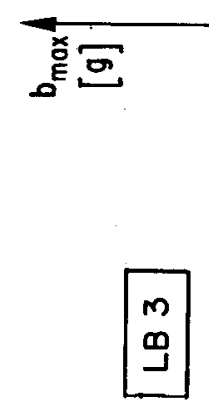

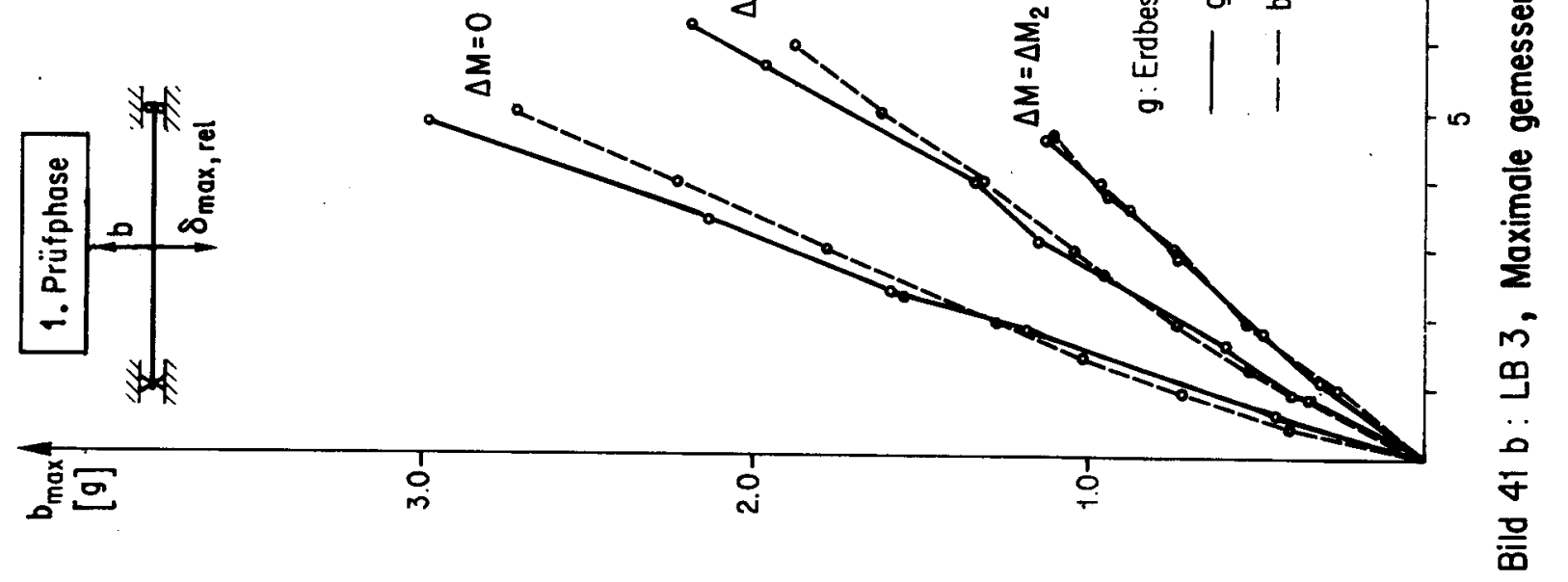




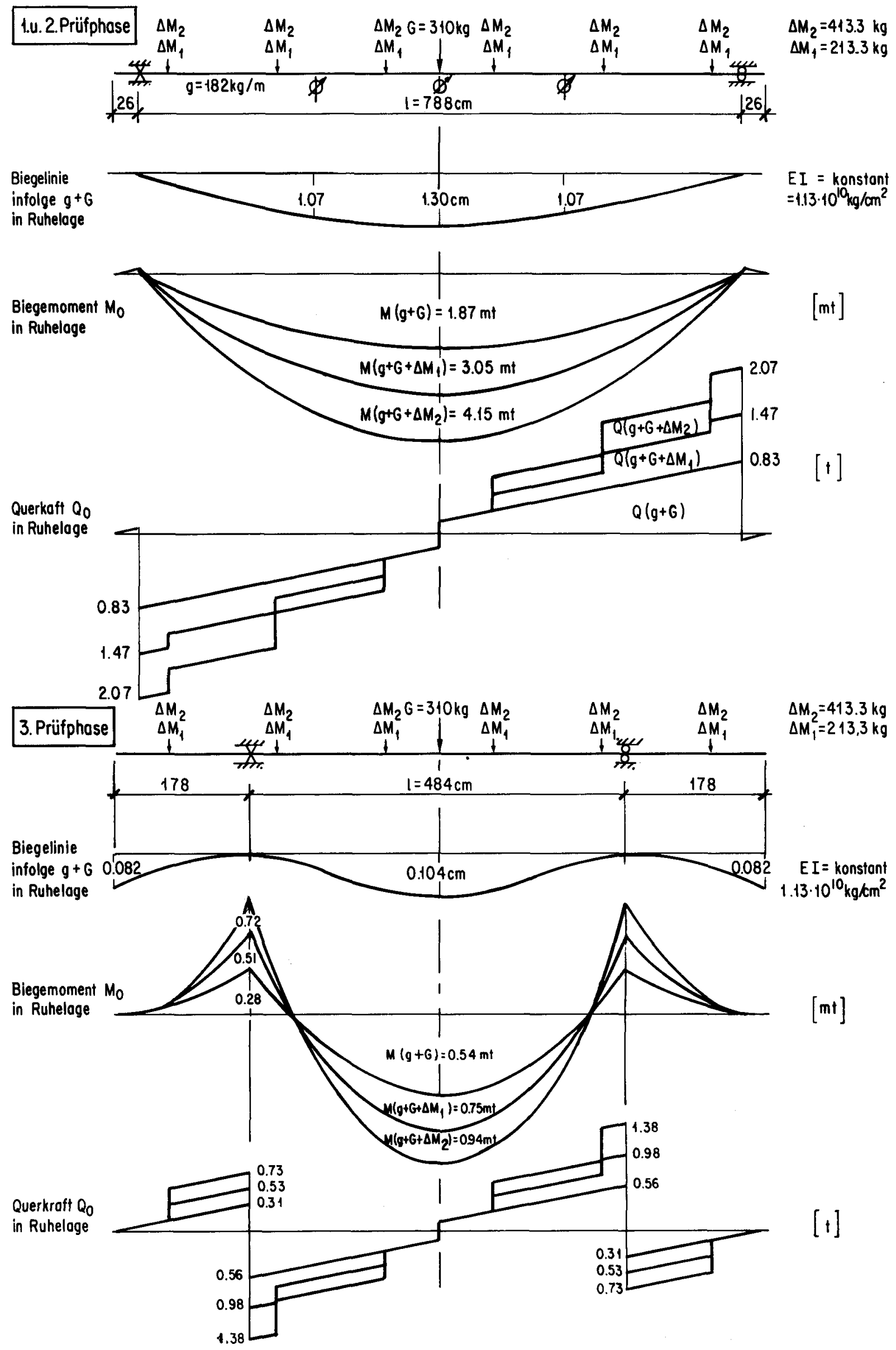

Bild 42 a: LB 3, Biege - und Querkraftbeanspruchung längs Balkenachse in Ruhelage 

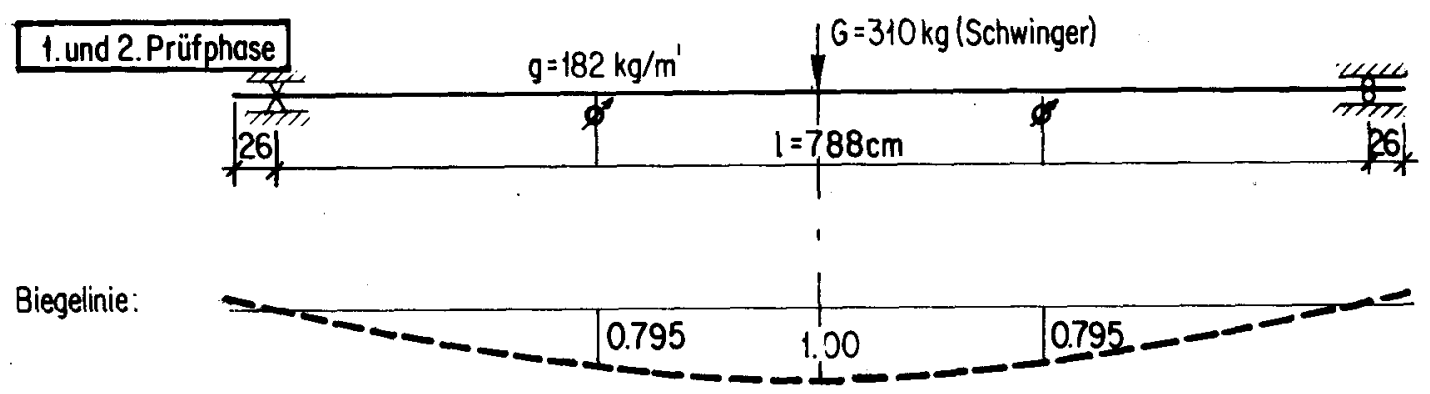

Dynamische Biegebeanspruchung bei einer Durchbiegung von $10 \mathrm{~cm}$ und einer Frequenz von $1.0 \mathrm{~Hz}$

Dynamische Quer kroftbeanspruchung bei einer Durchbiegung von $10 \mathrm{~cm}$ und einer Frequen $z$ von $1.0 \mathrm{~Hz}$

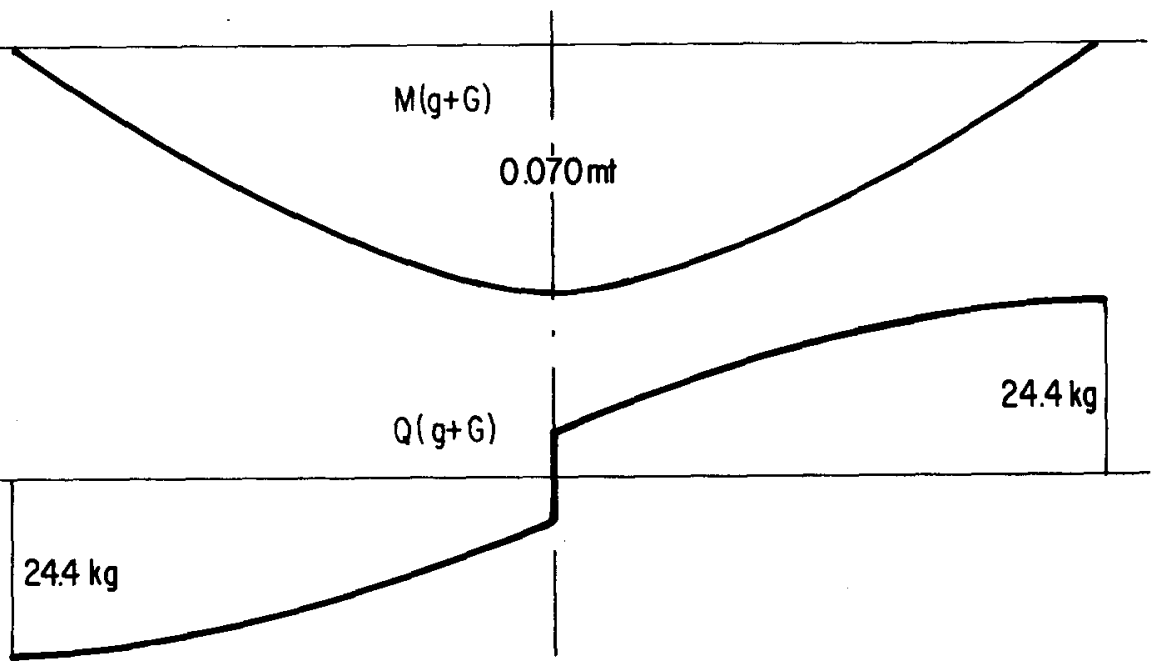

Foktor $\delta_{\operatorname{mox}, r^{f} f^{f^{2}}}$ $[\mathrm{cm}]\left[\left(\frac{1}{s}\right)^{2}\right]$
Faktor

$\delta_{\text {max, ret }} t^{2}$ $[\mathrm{cm}]\left[\left(\frac{1}{s}\right)^{2}\right]$

\section{LB 3}

\section{Prüfphase}

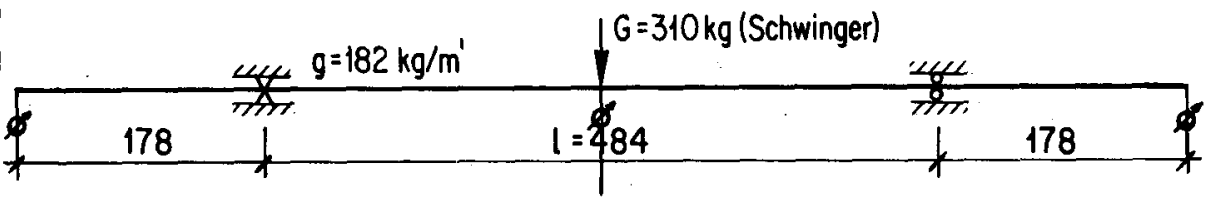

Biegelinie:

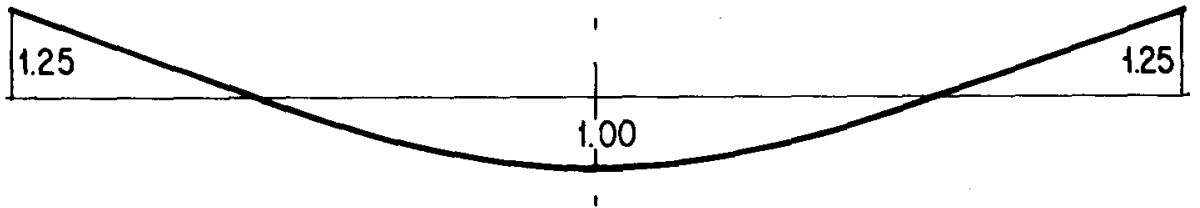

Dynamische Biegebeanspruchung be einer Durchbiegung von $10 \mathrm{~cm}$ und einer Frequenz von $1.0 \mathrm{~Hz}$

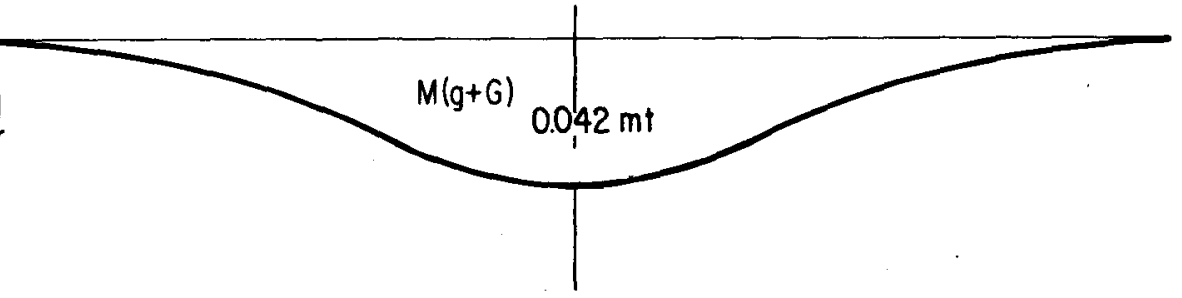

Foktor

$\delta_{\text {max }}$ re $\mathrm{f}^{\mathrm{f}^{2}}$ $[\mathrm{cm}]\left[\left(\frac{1}{5}\right)^{2}\right]$

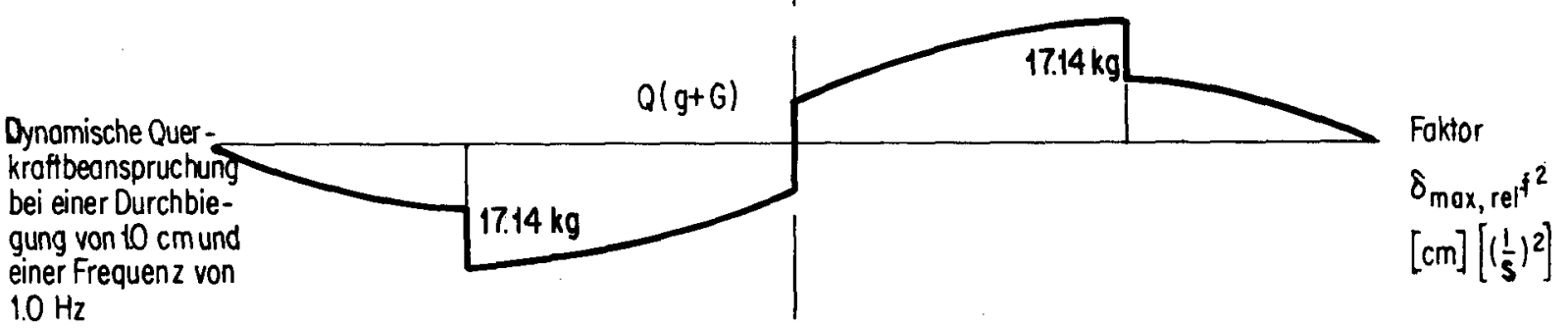

Bild 42b: LB 3, $\Delta M=0$, Relative Biege - und Querkraftbeanspruchung längs Balkenachse bei dynamischer Belastung 


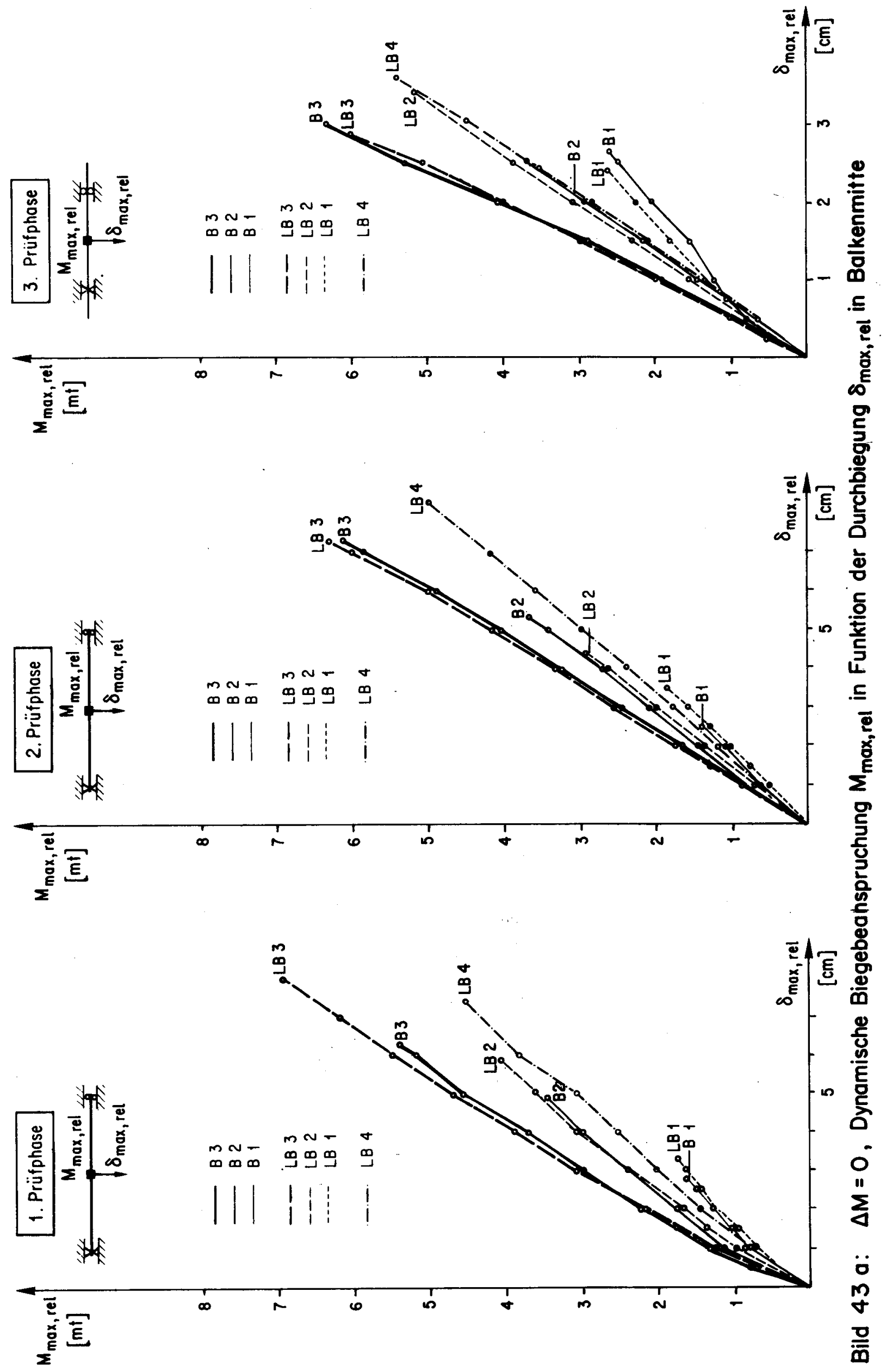




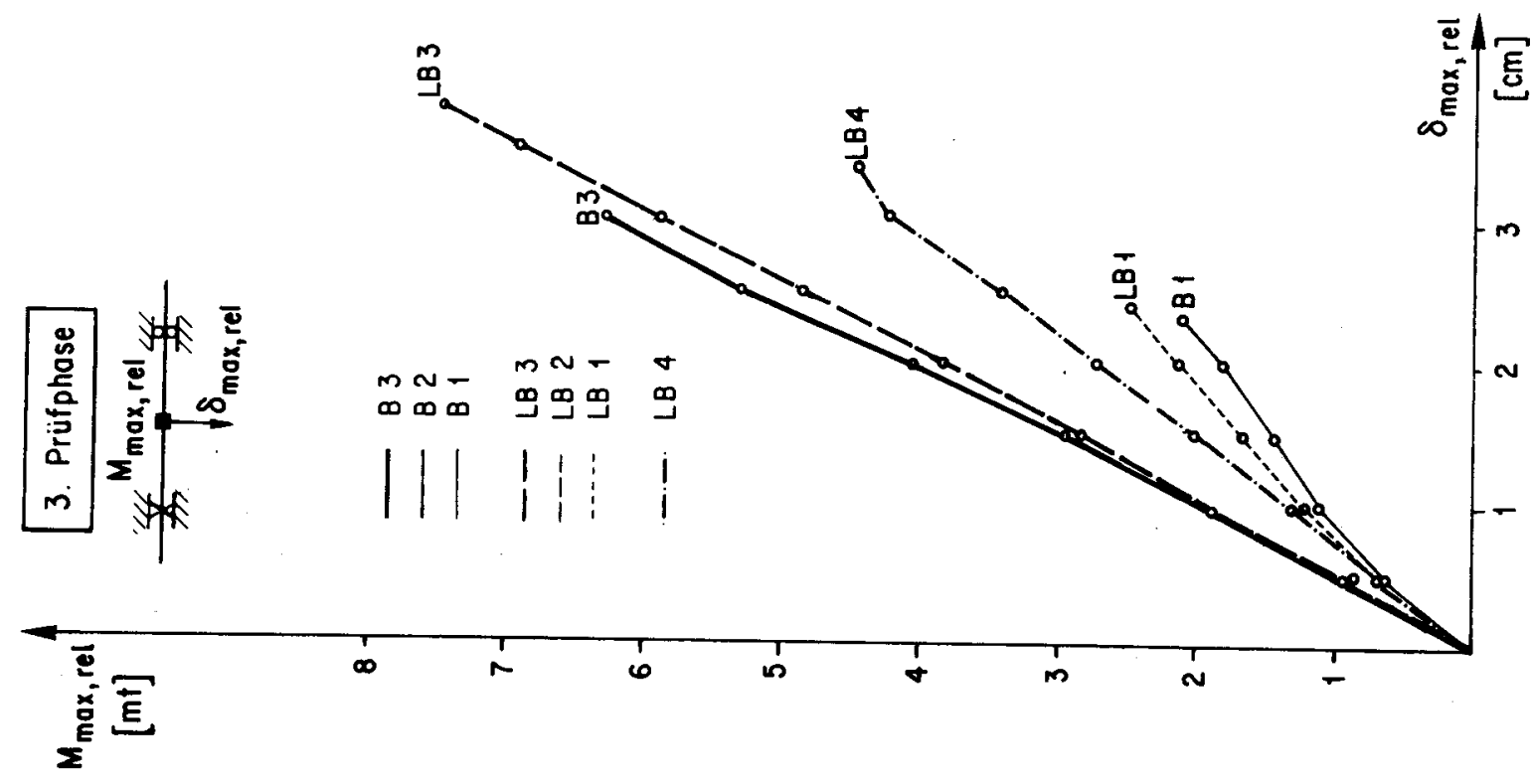

焉
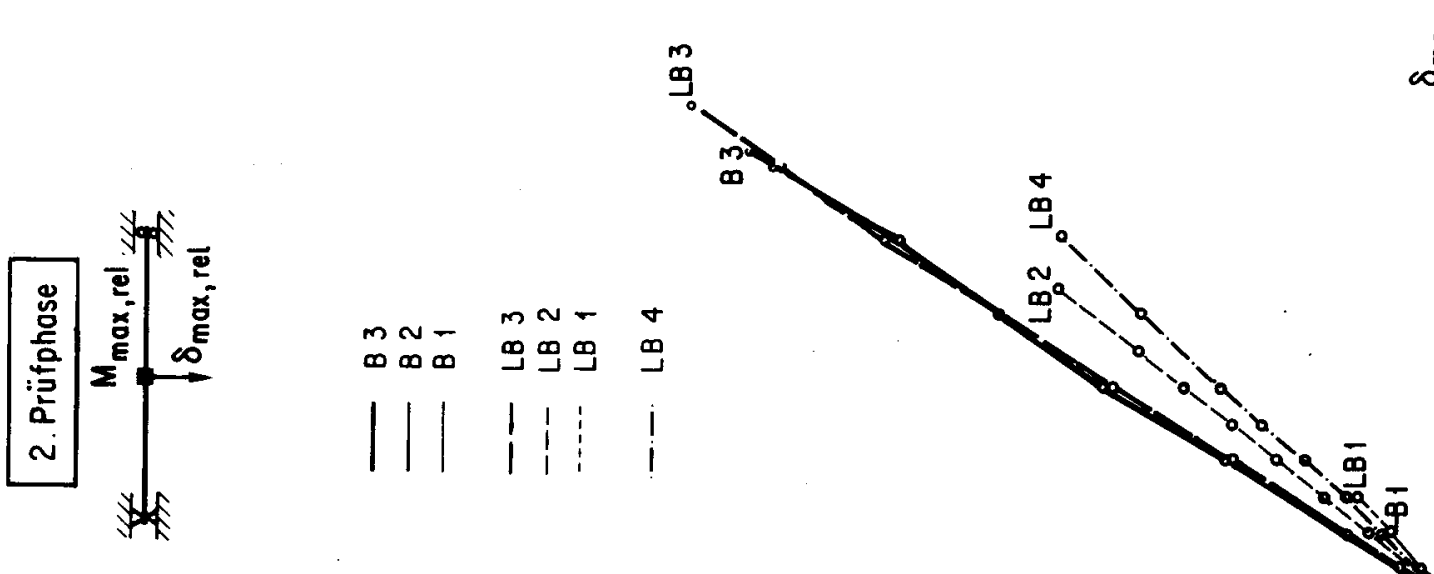

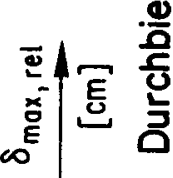
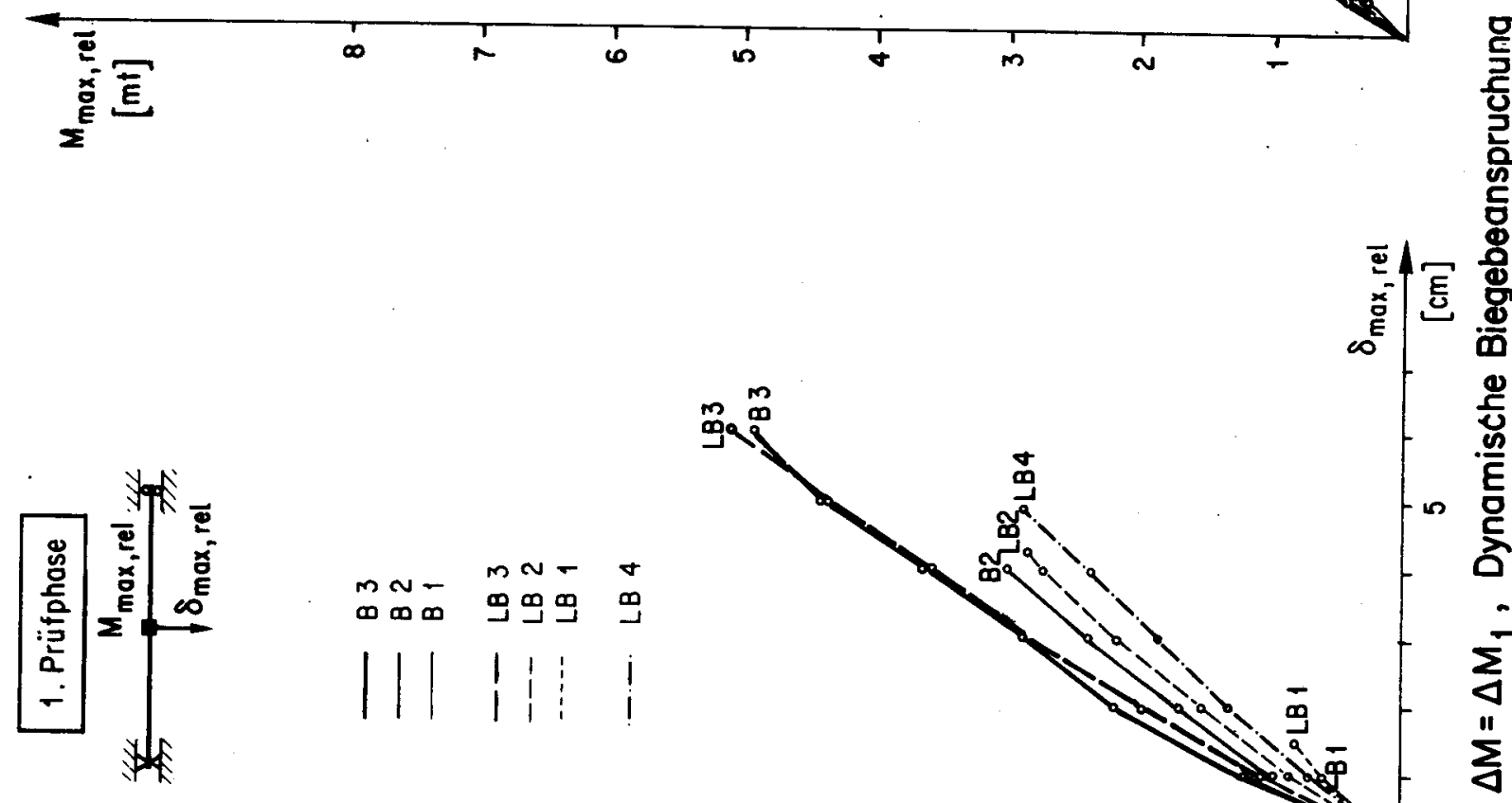

突

通

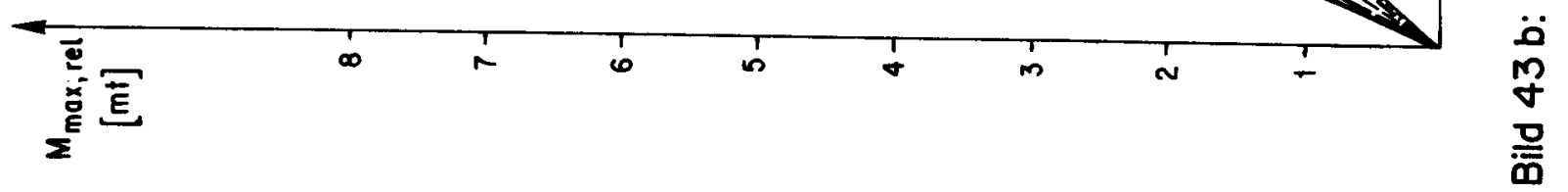



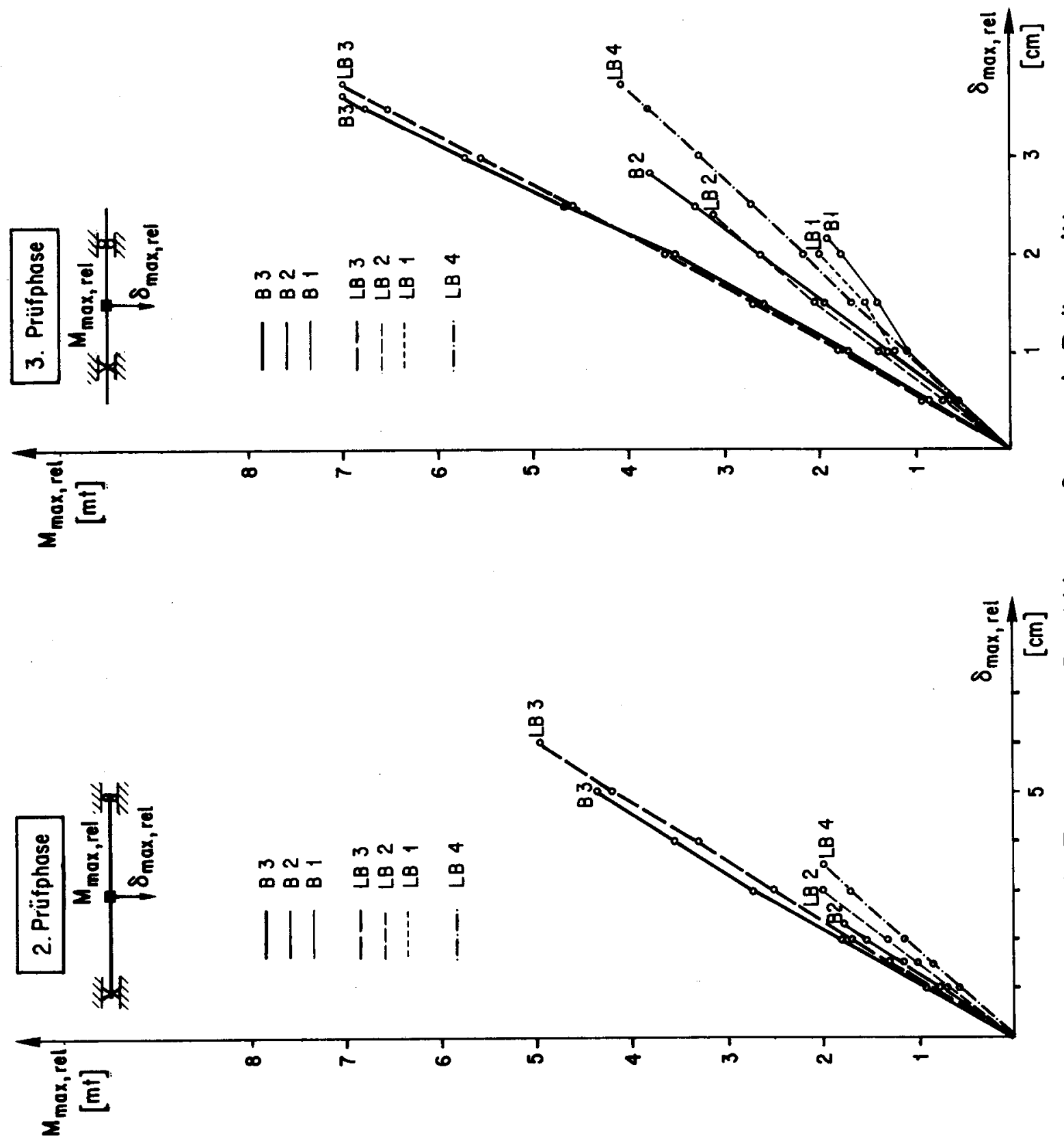

ฆ্ํ

음

号
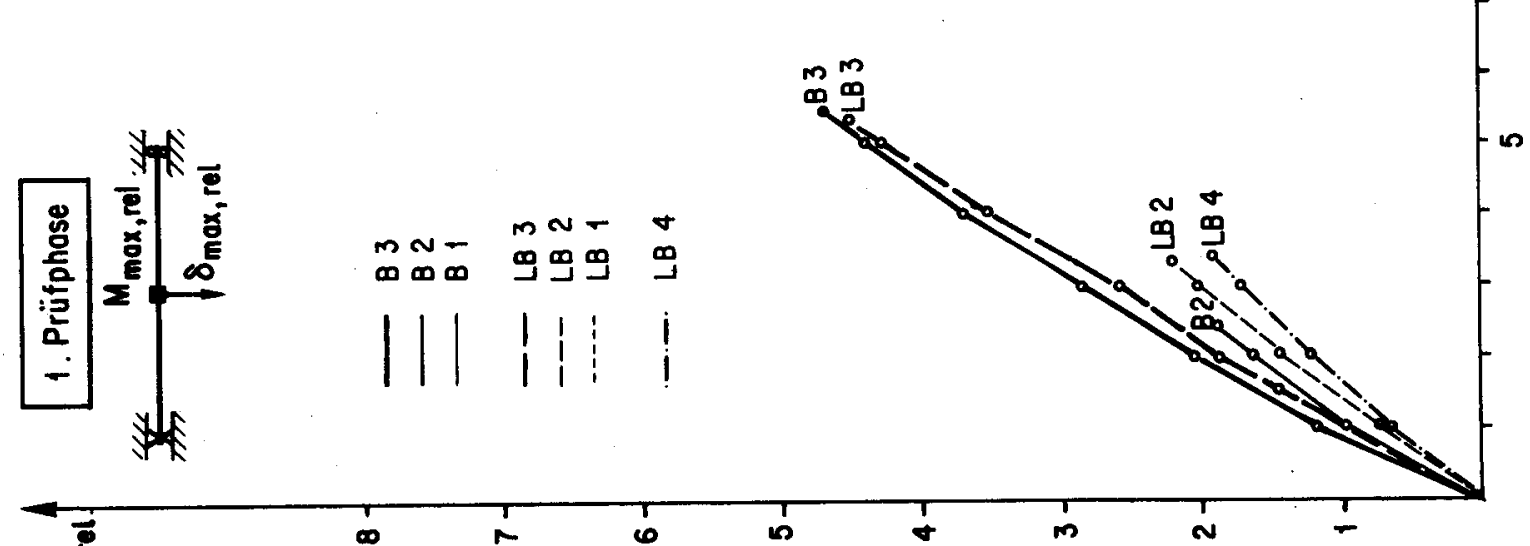

$\frac{y}{u}$
$\frac{.}{E}$
$\frac{0}{\delta}$

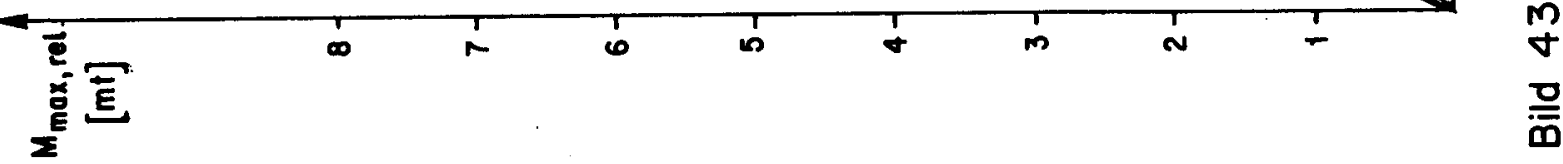



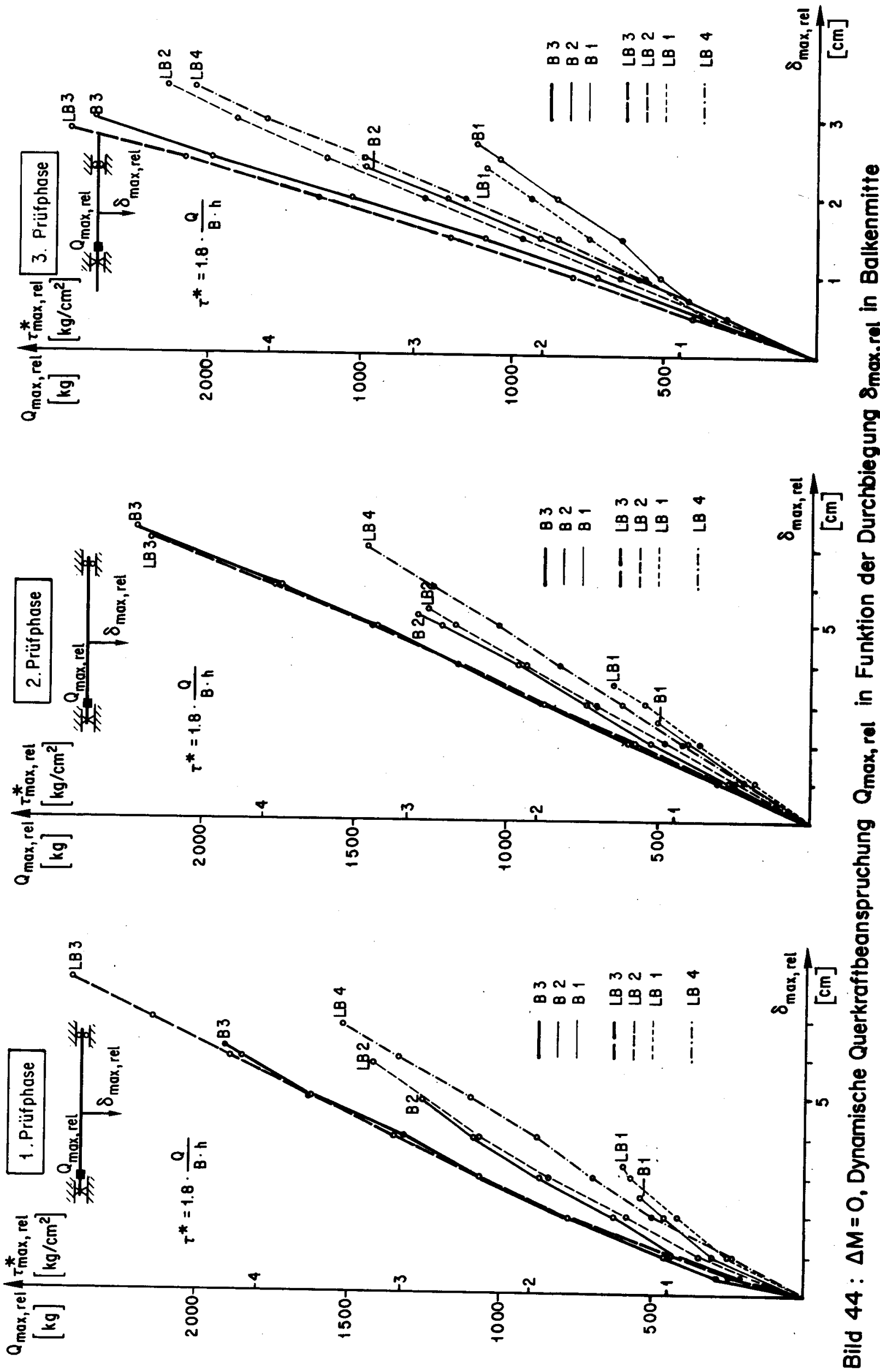

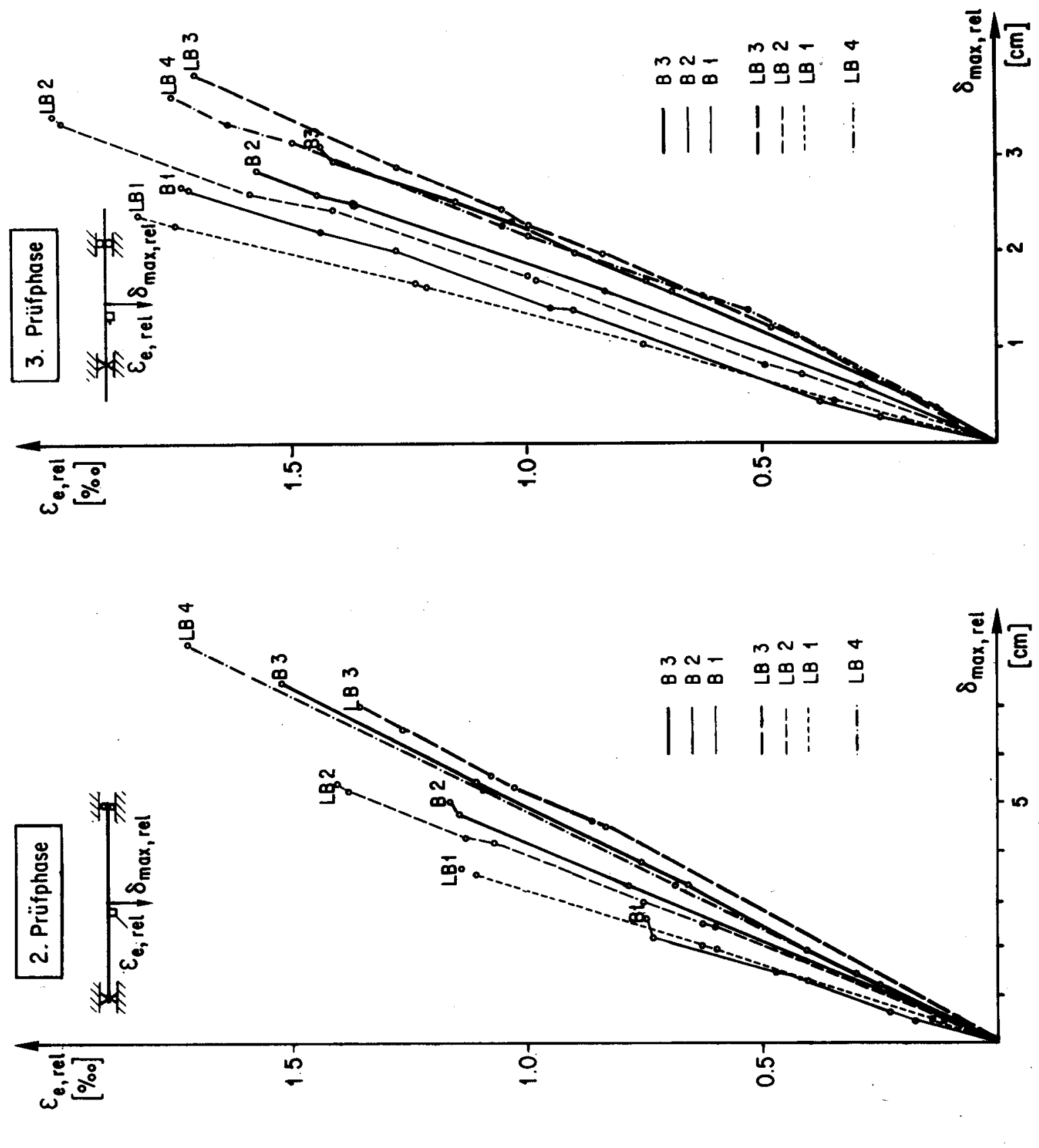

을

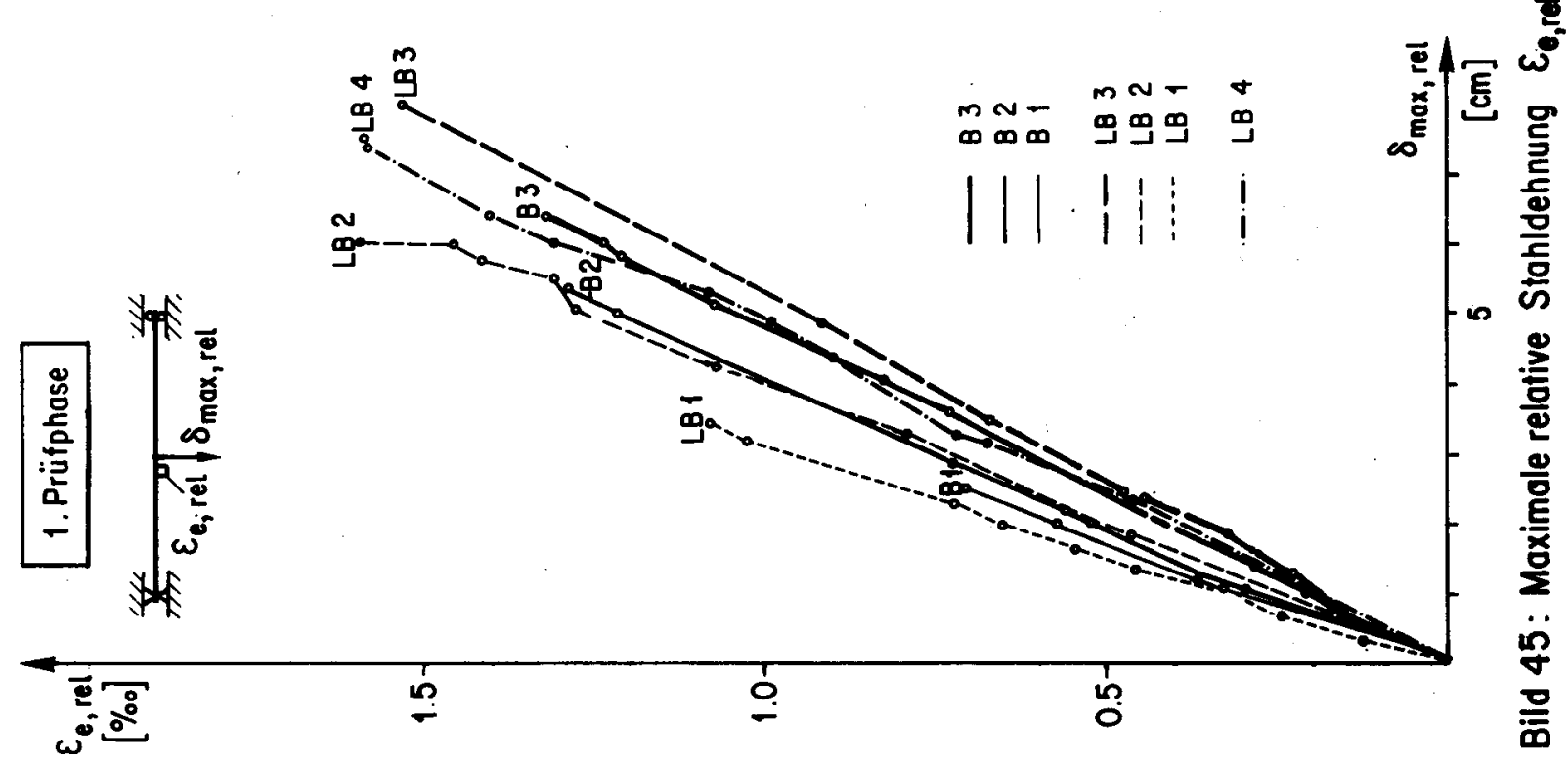




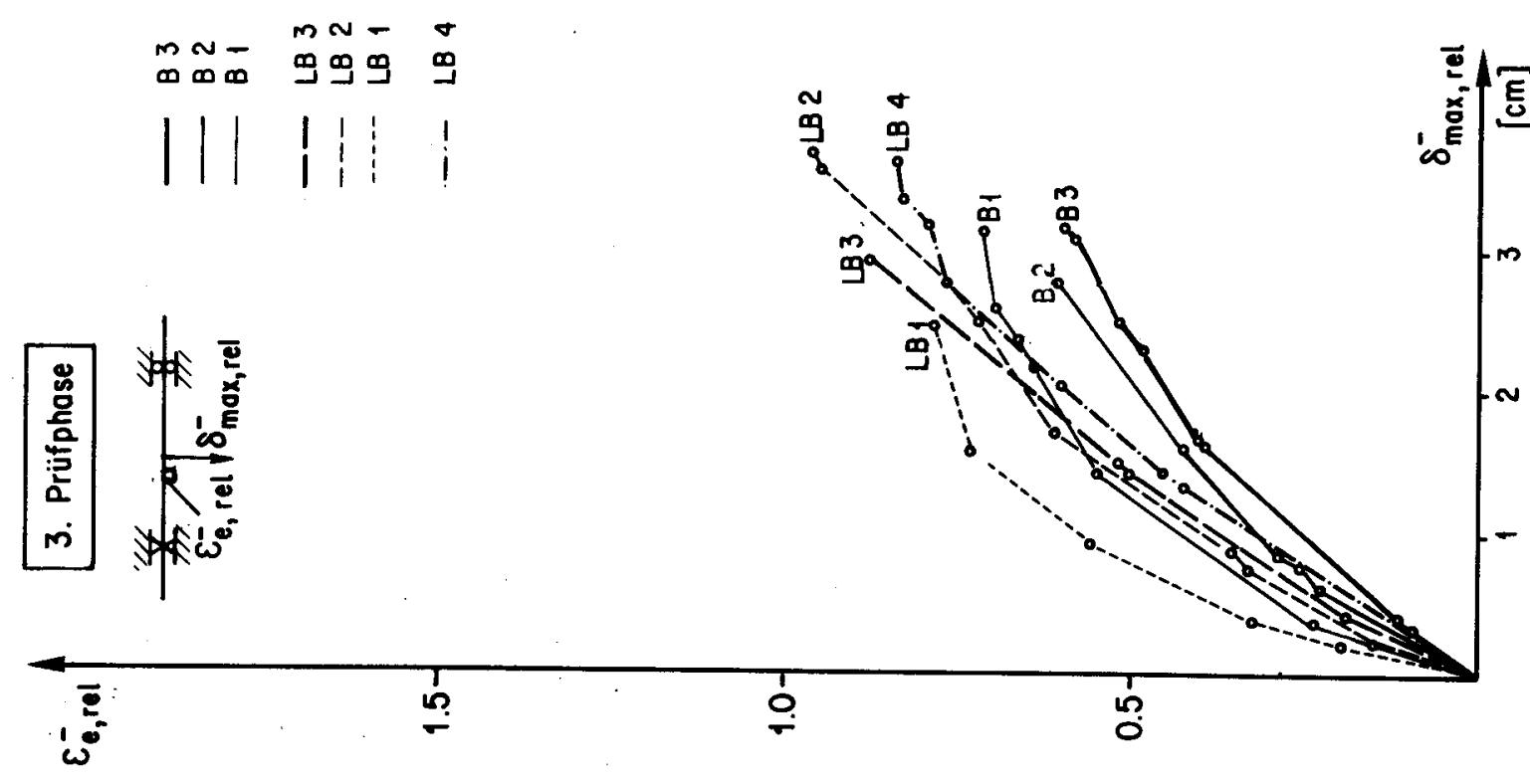

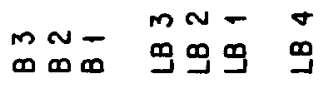

$111+1$
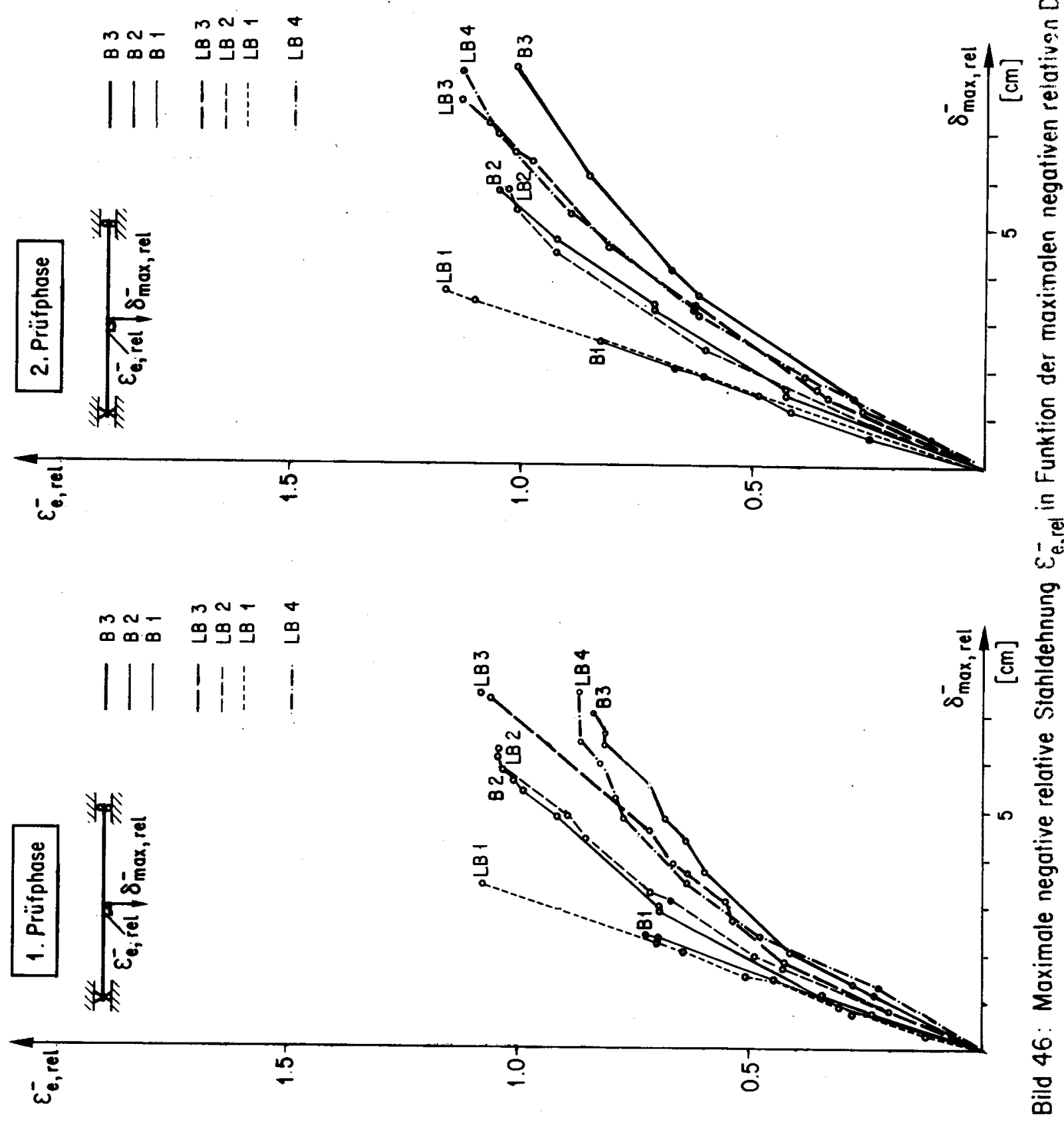

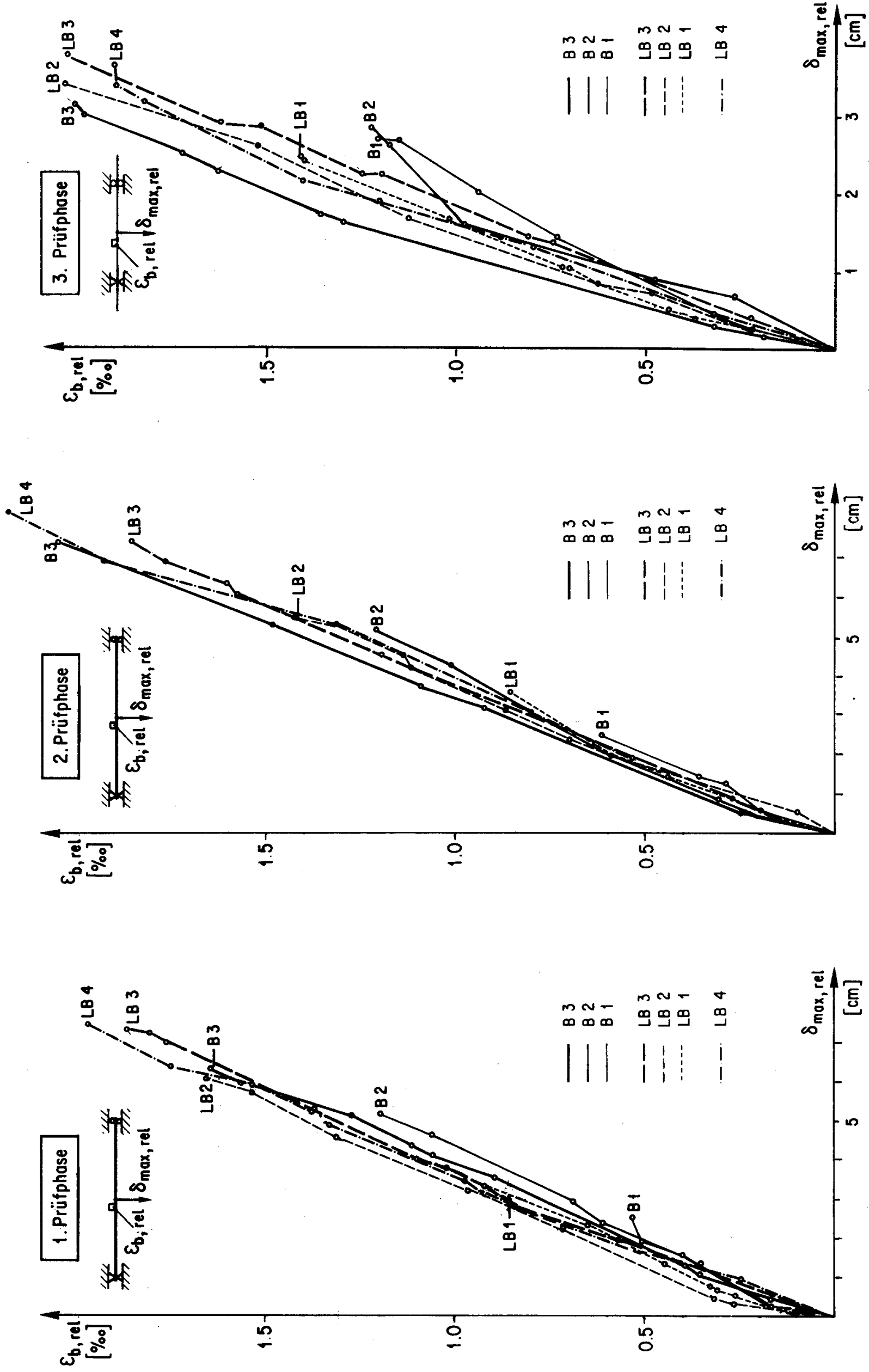

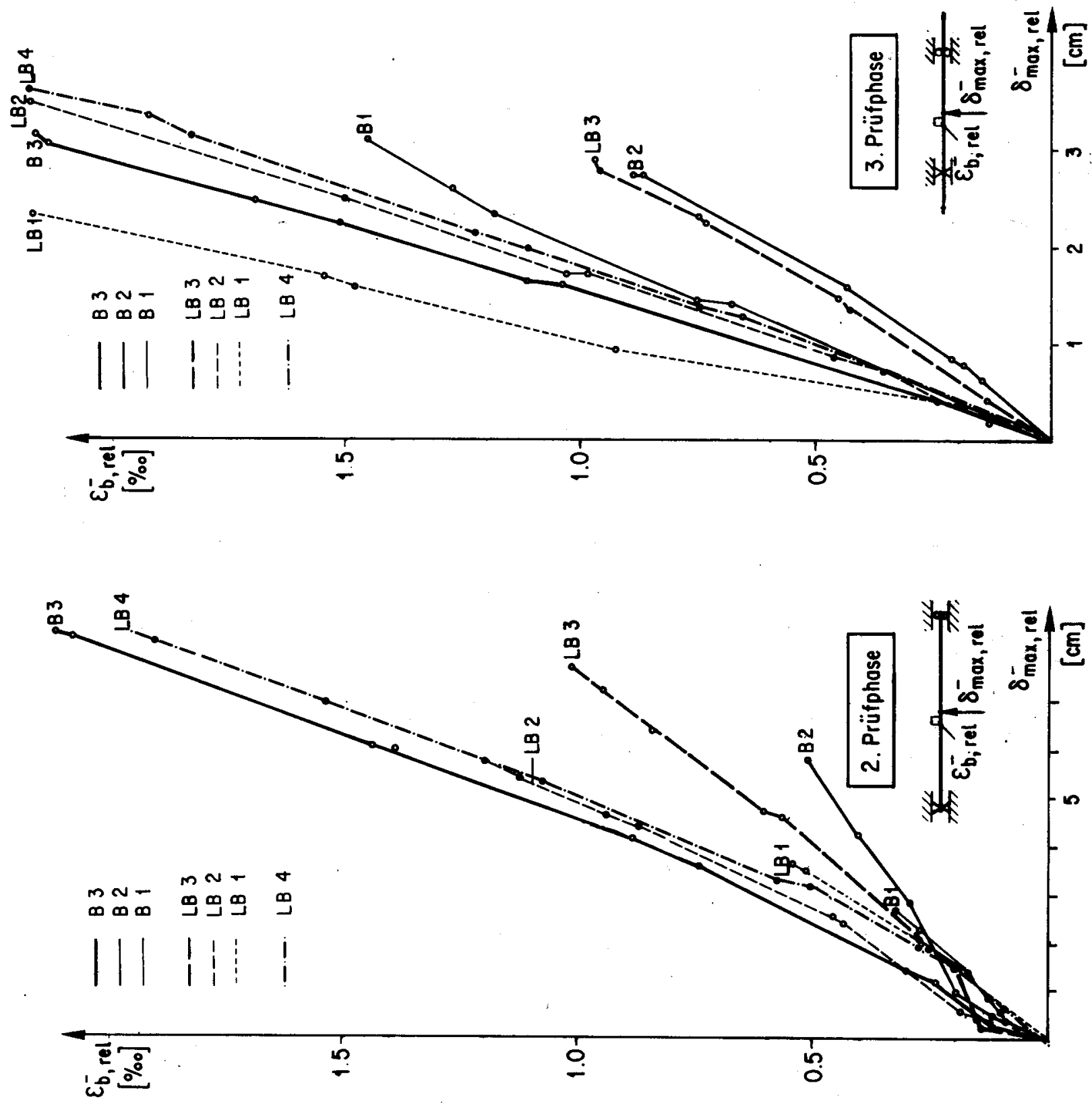

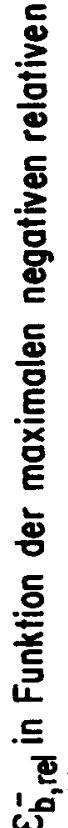

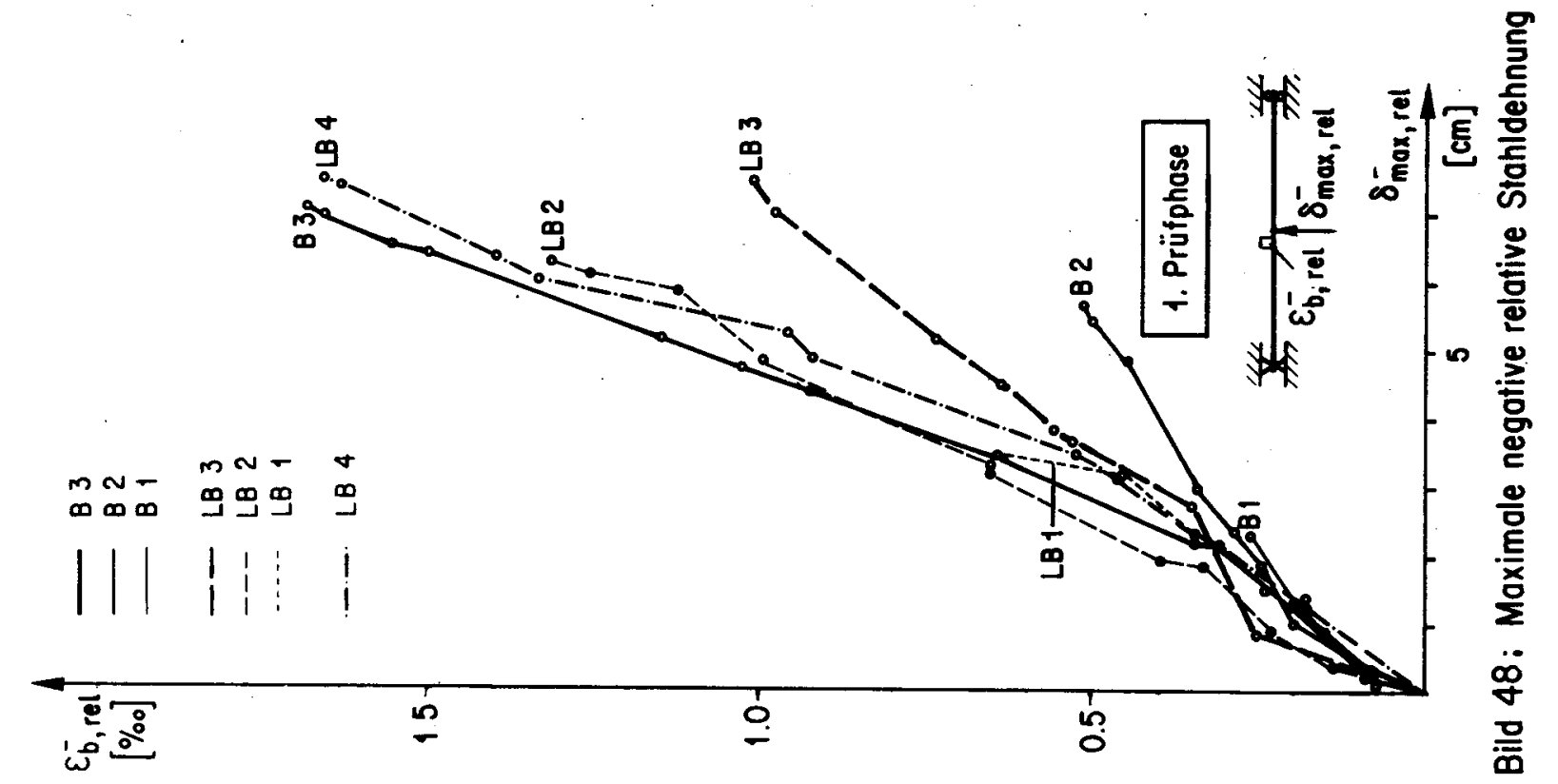




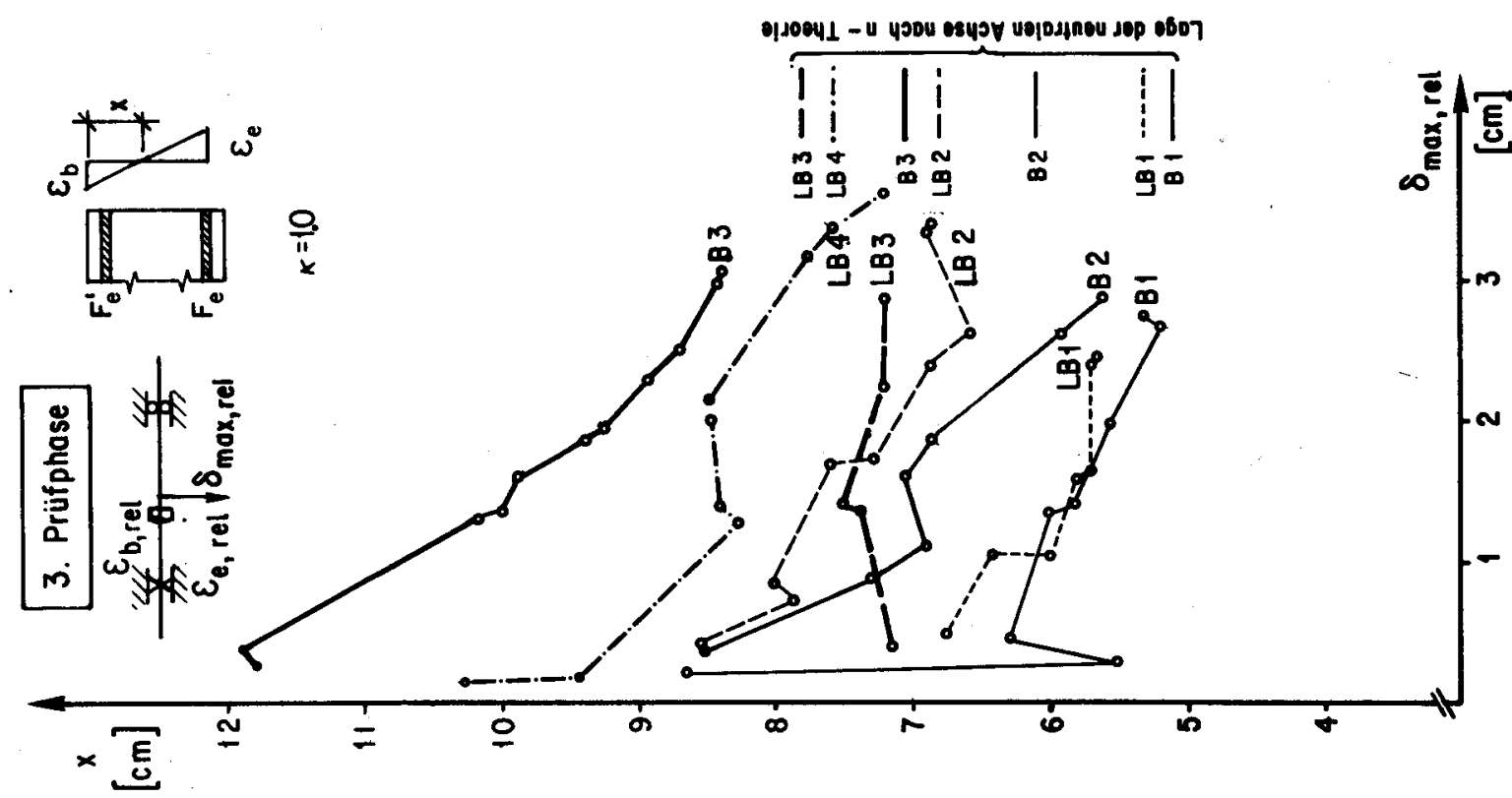

롱

$\omega^{+x}+\omega^{*}$

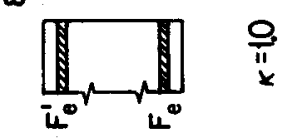



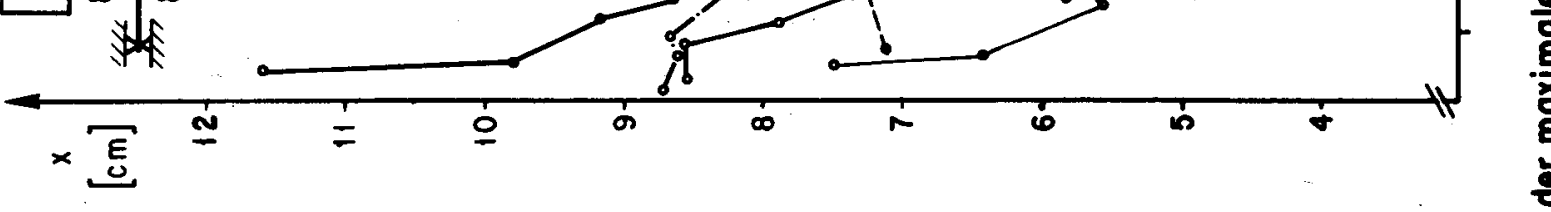

कt

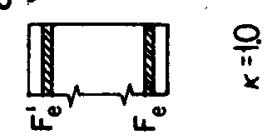

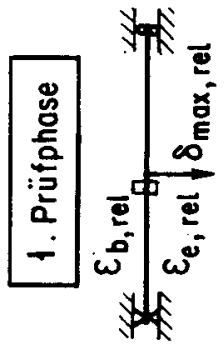



드 
|
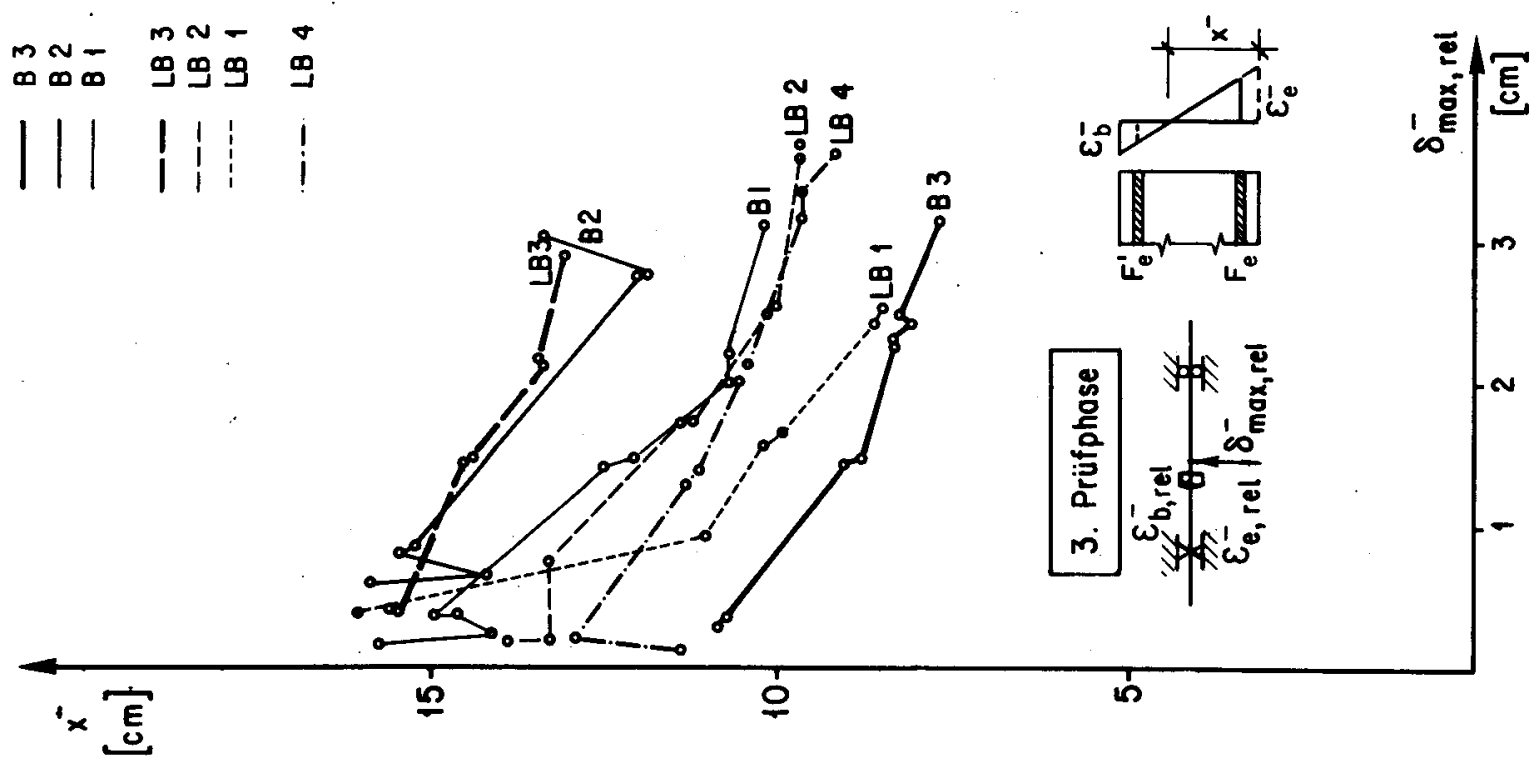

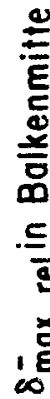

年
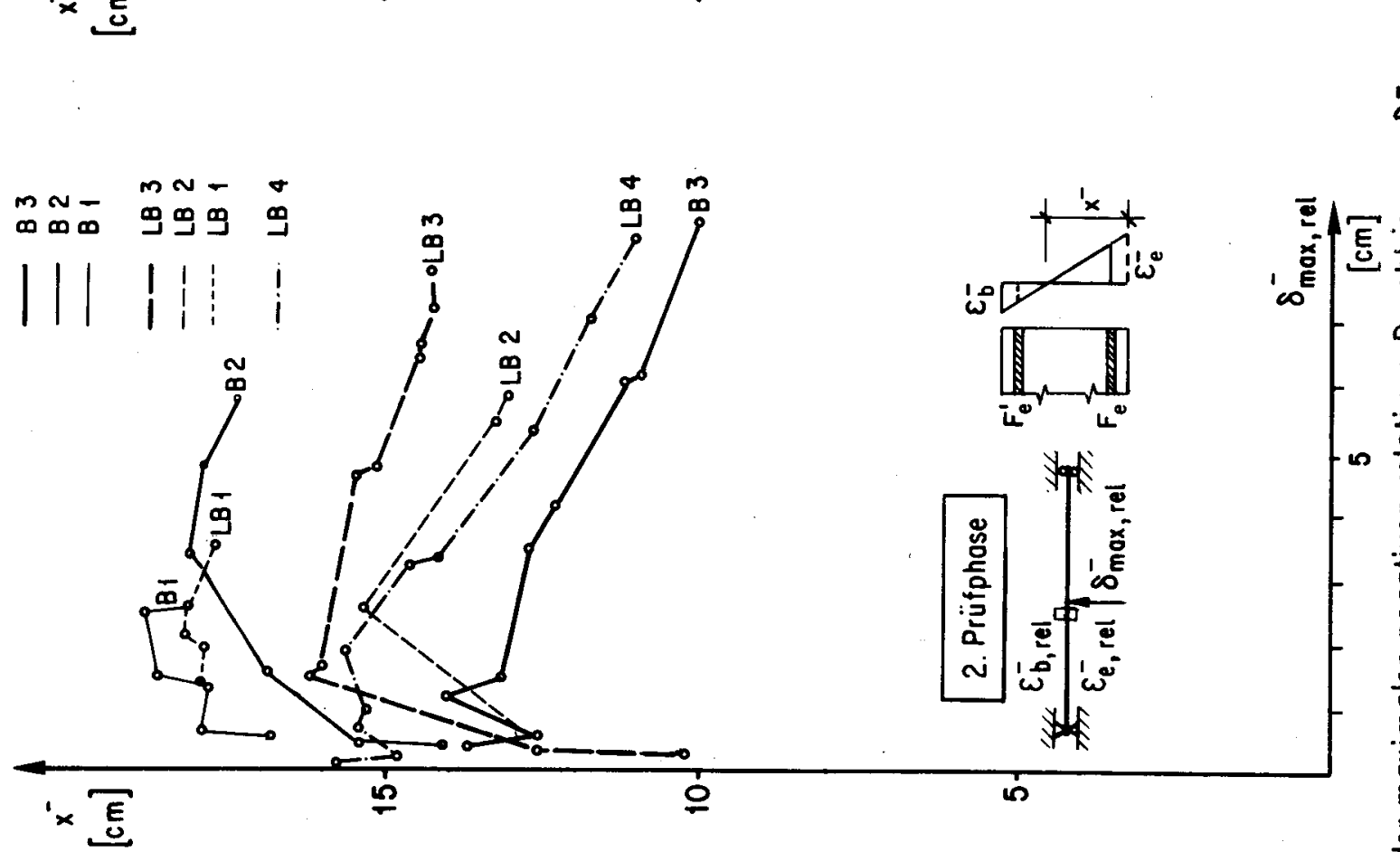

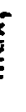



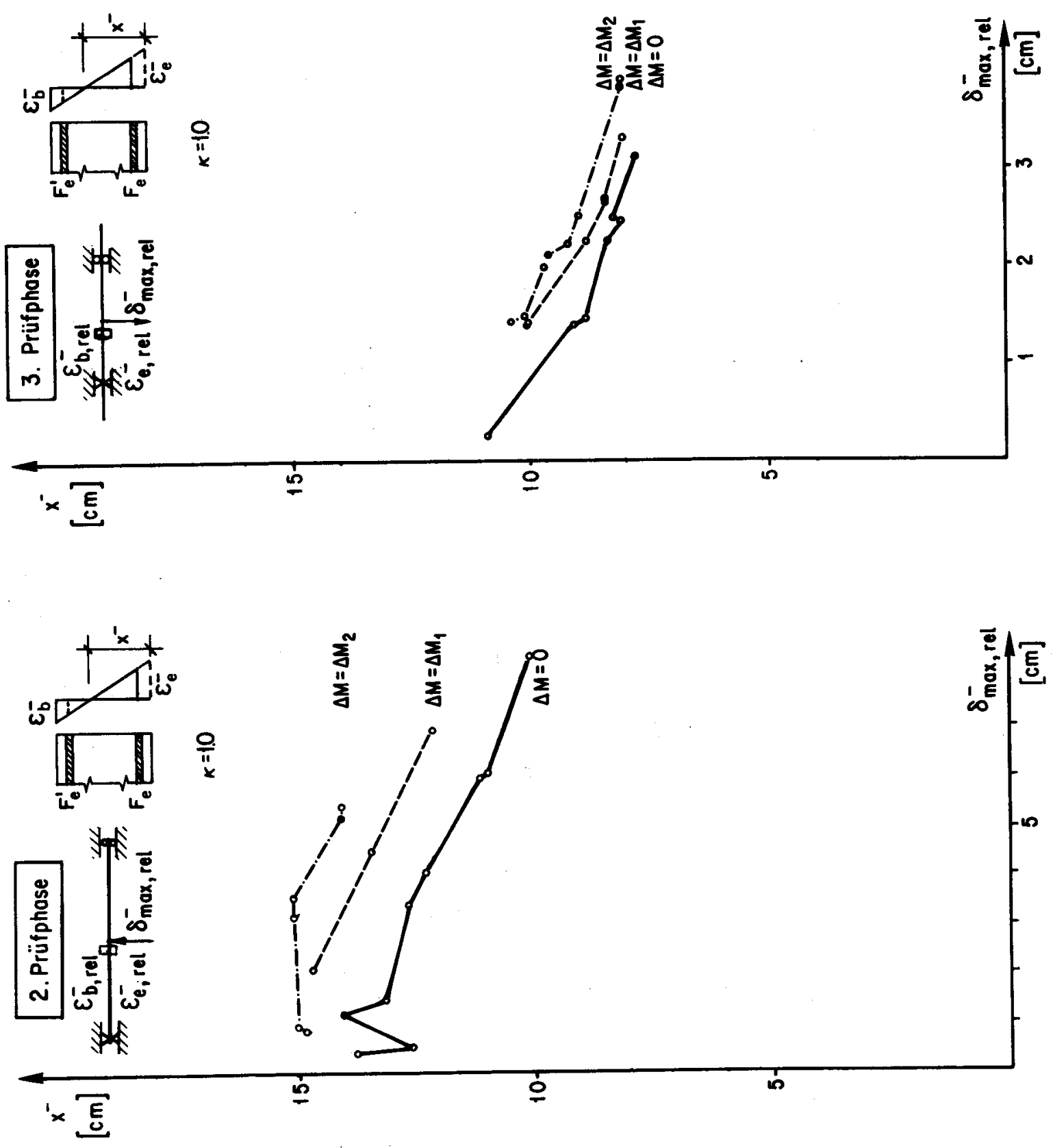

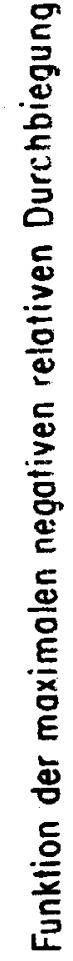

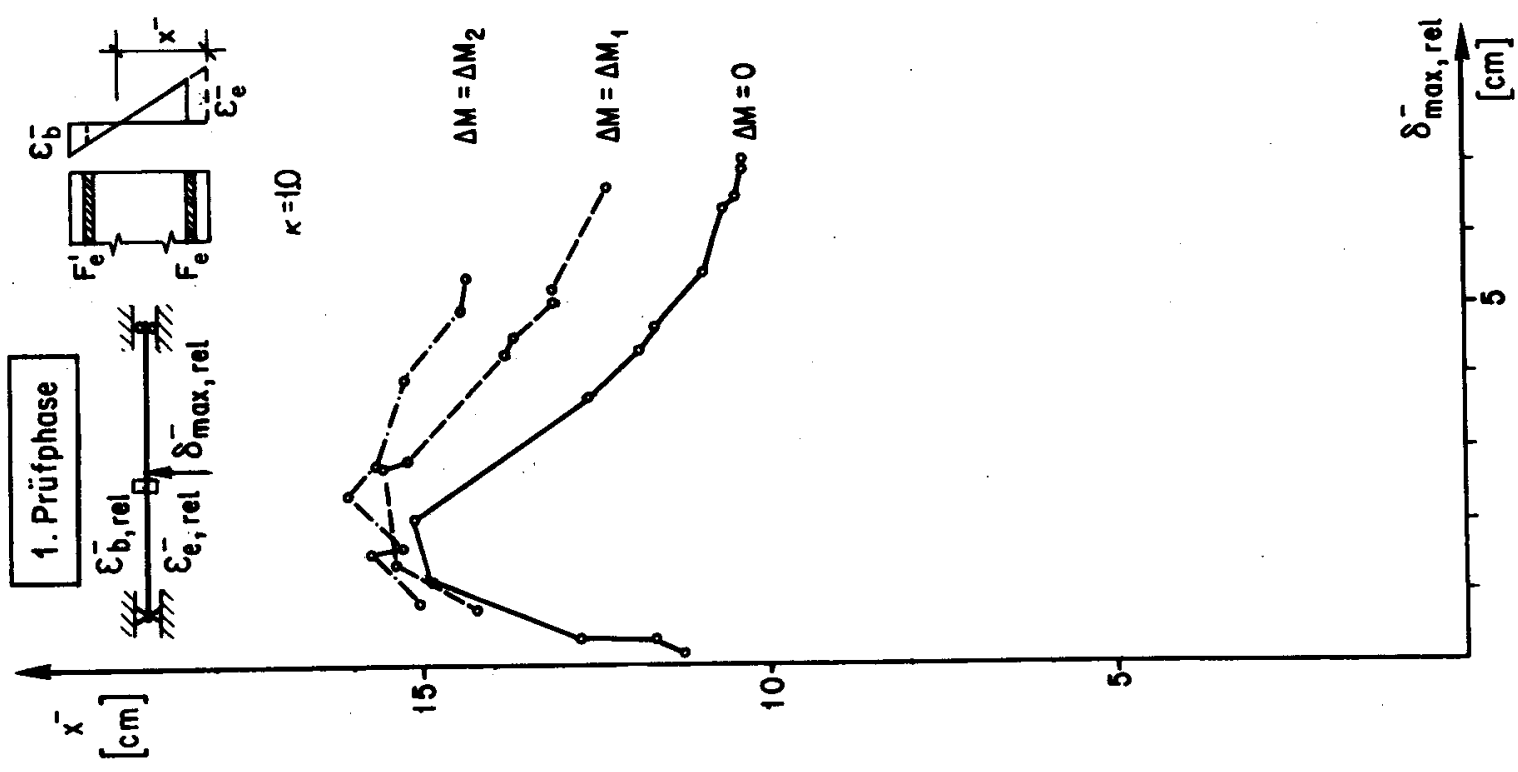






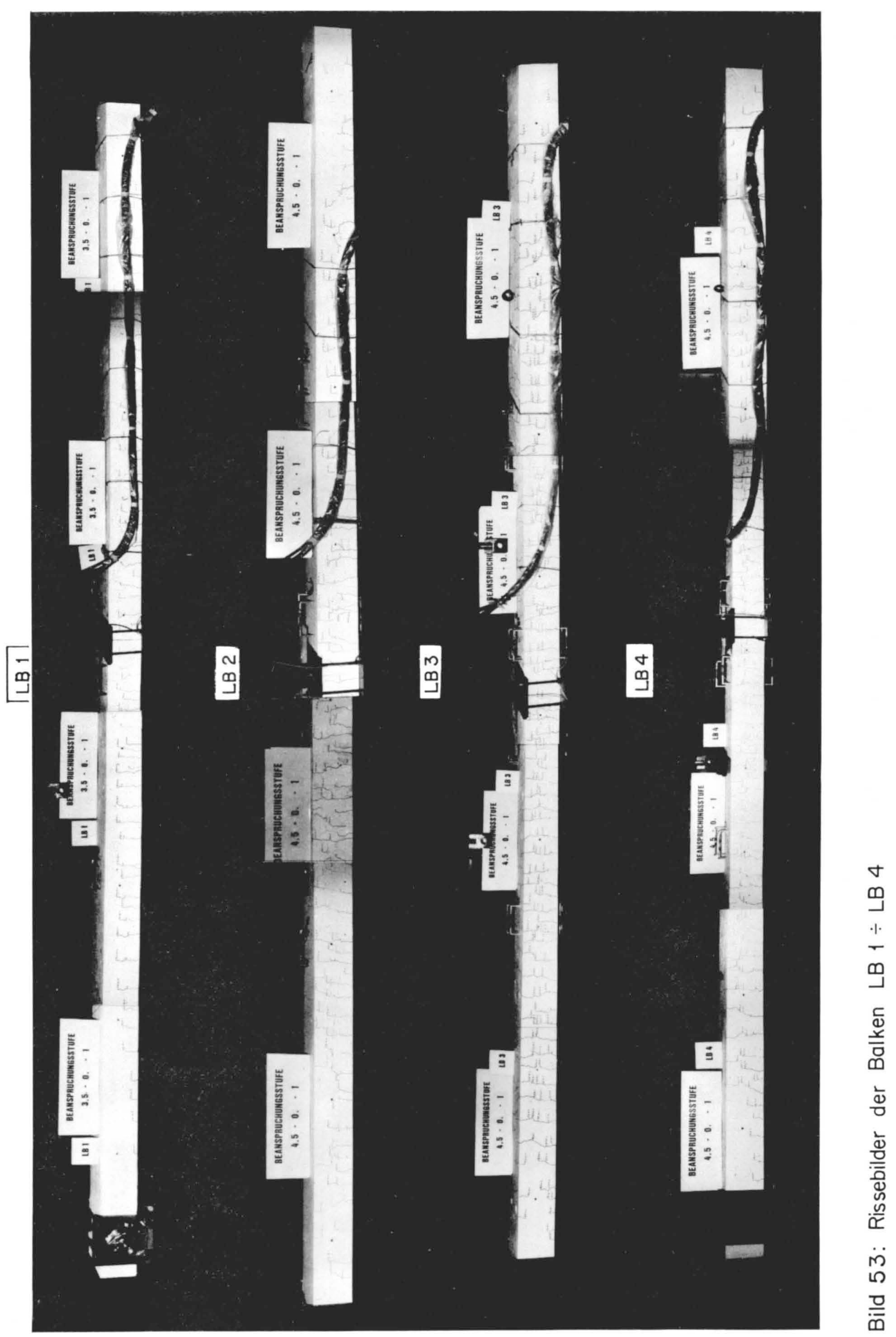



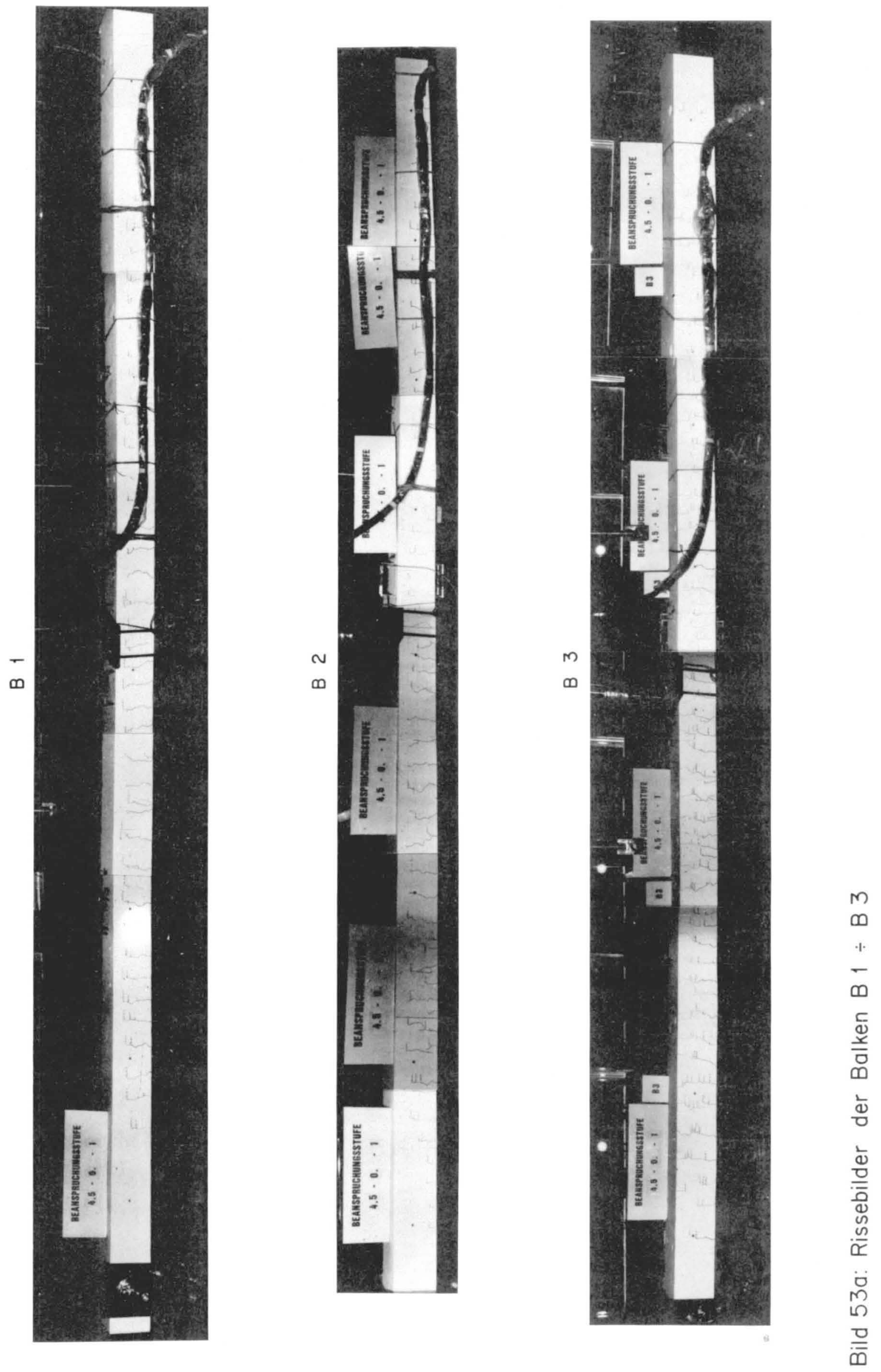


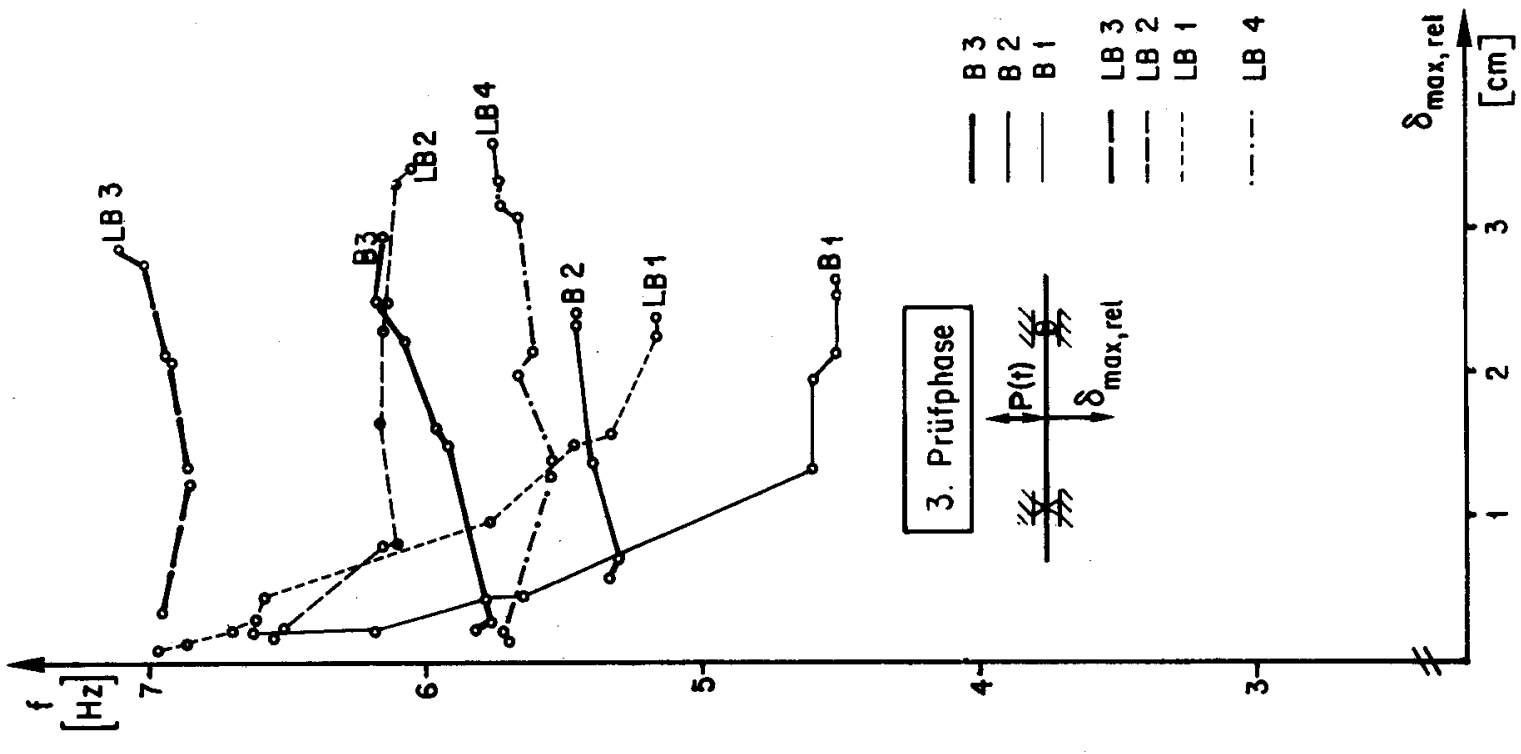

害

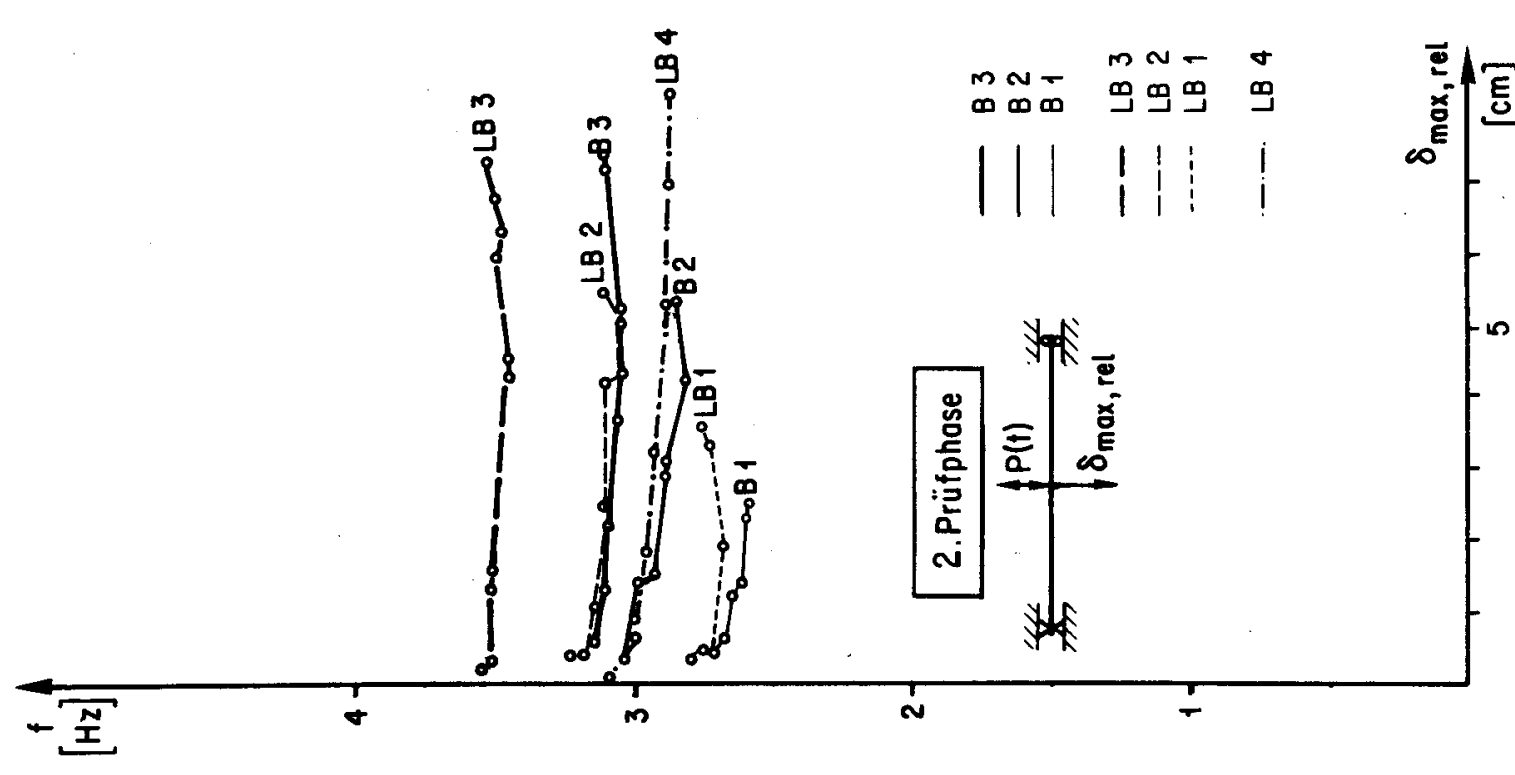

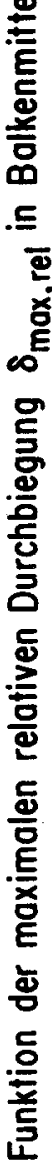

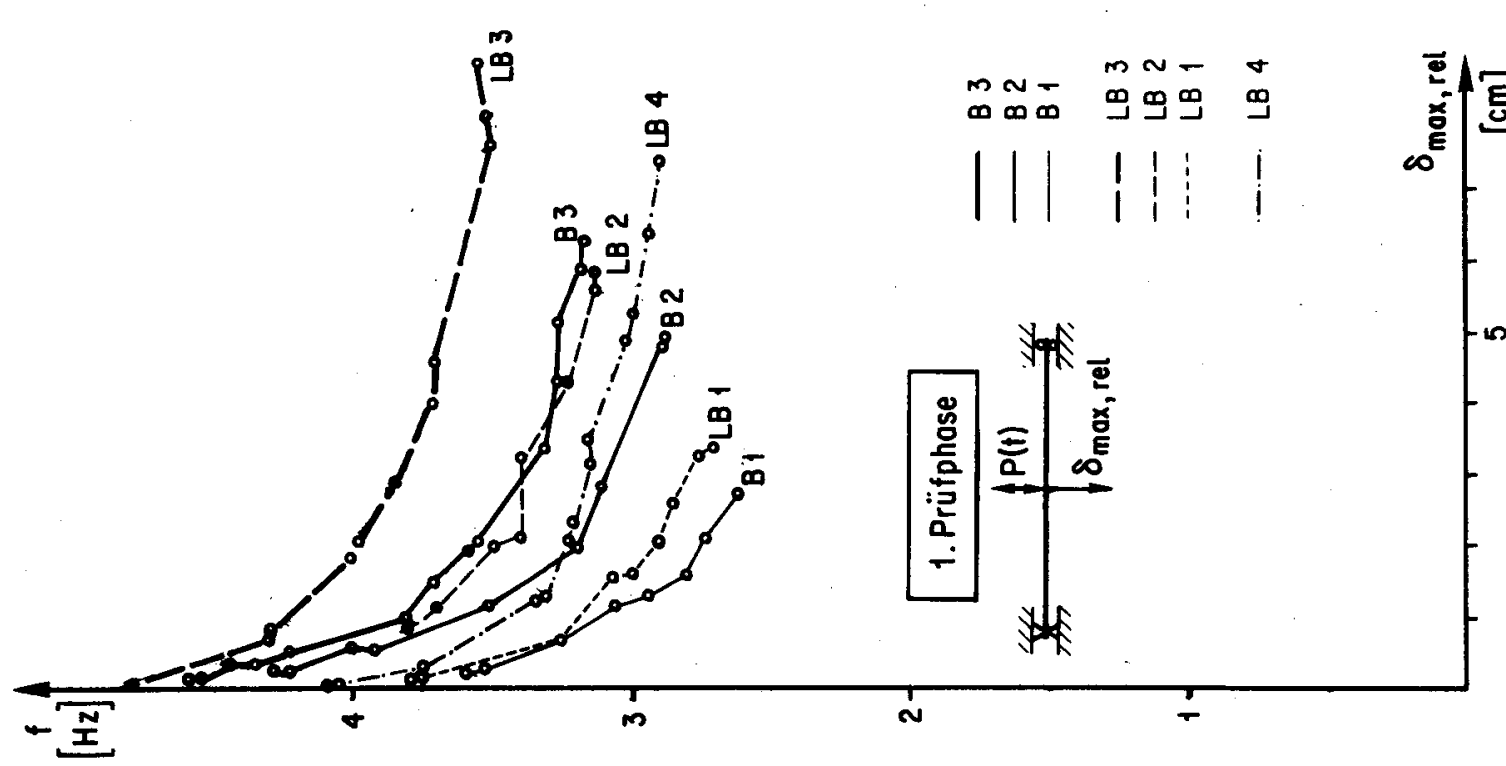

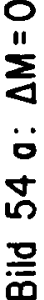




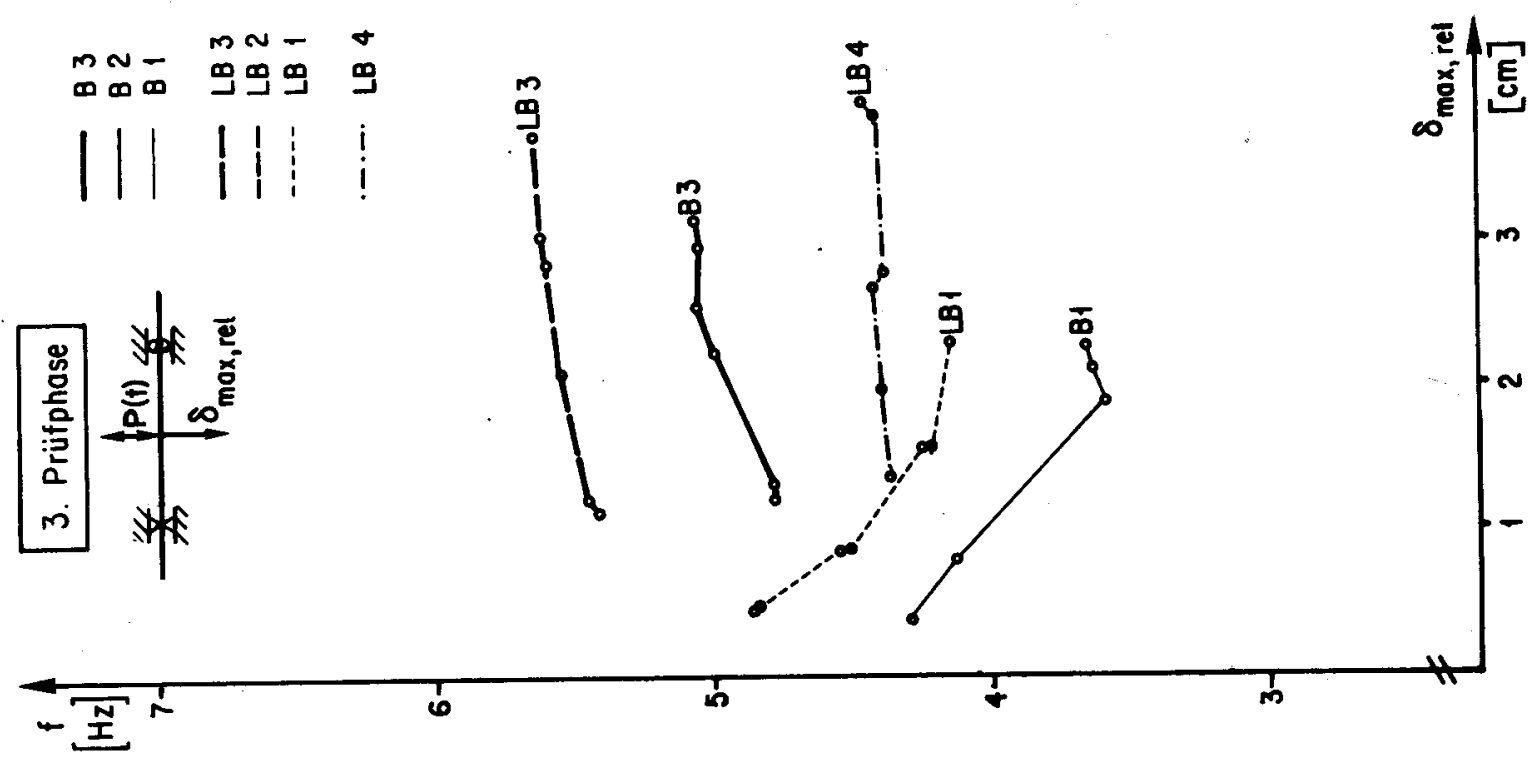

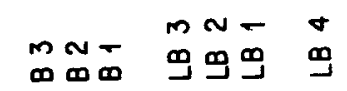

111 1 $11: 1$
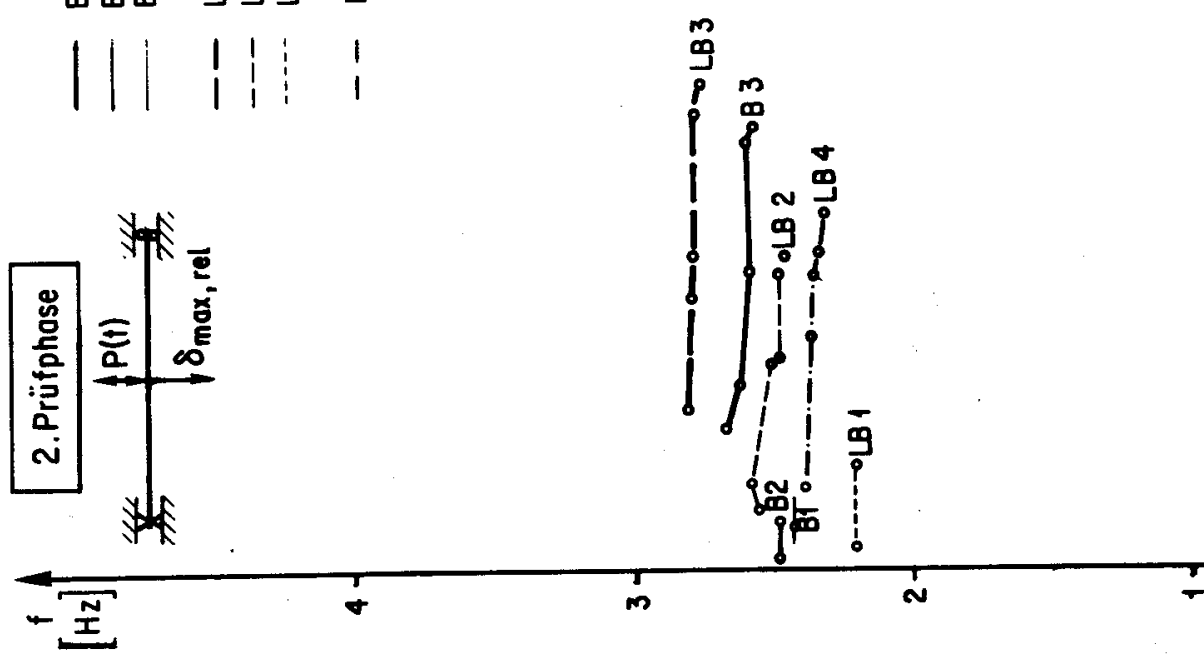

를
틍
흥
.

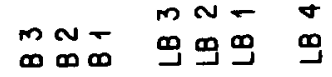

11111151
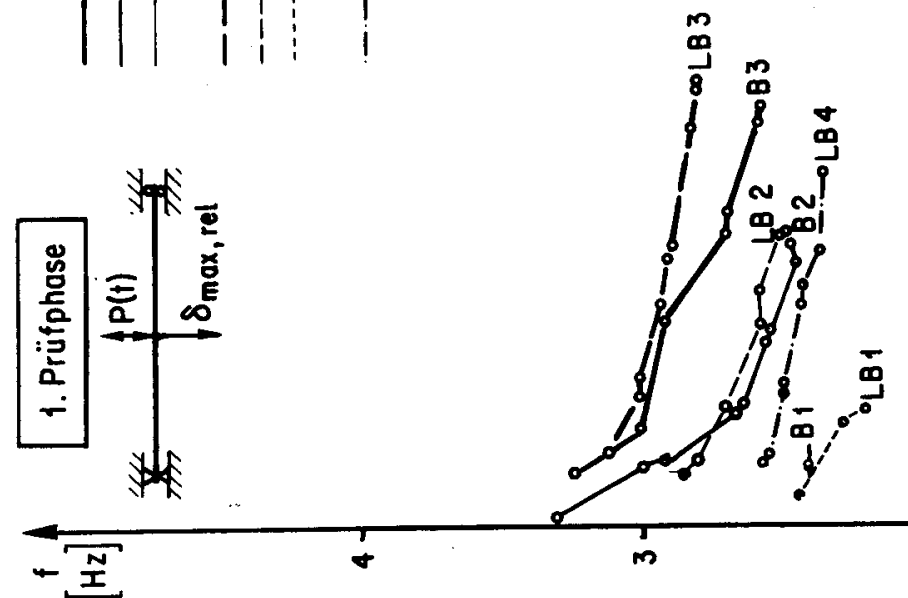






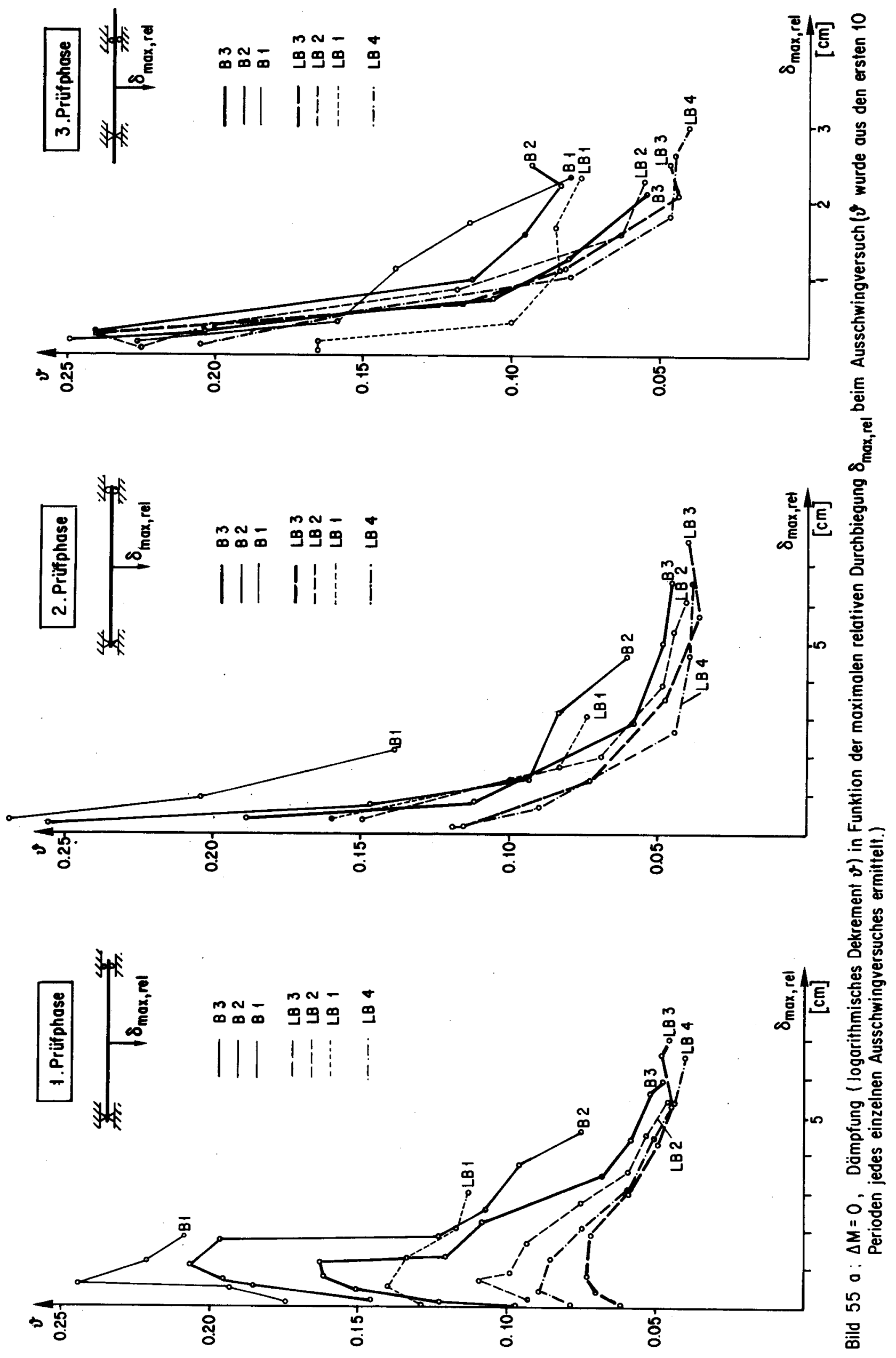


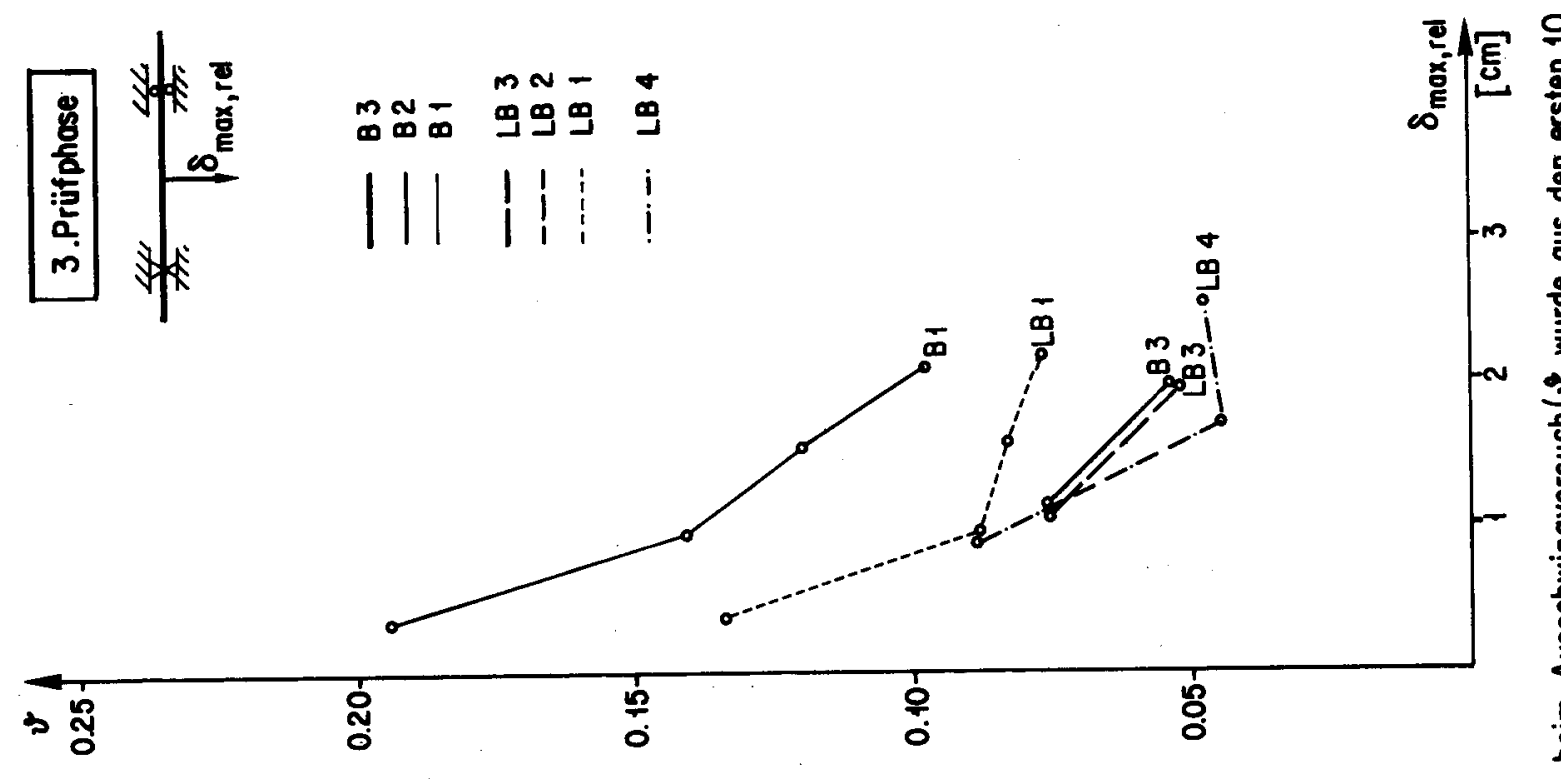

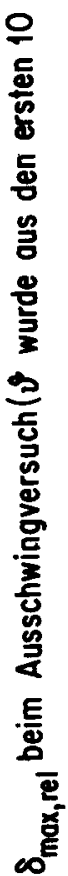

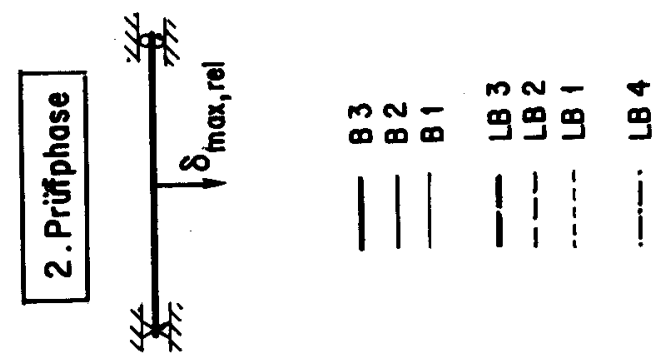

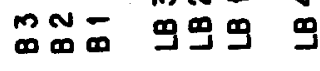

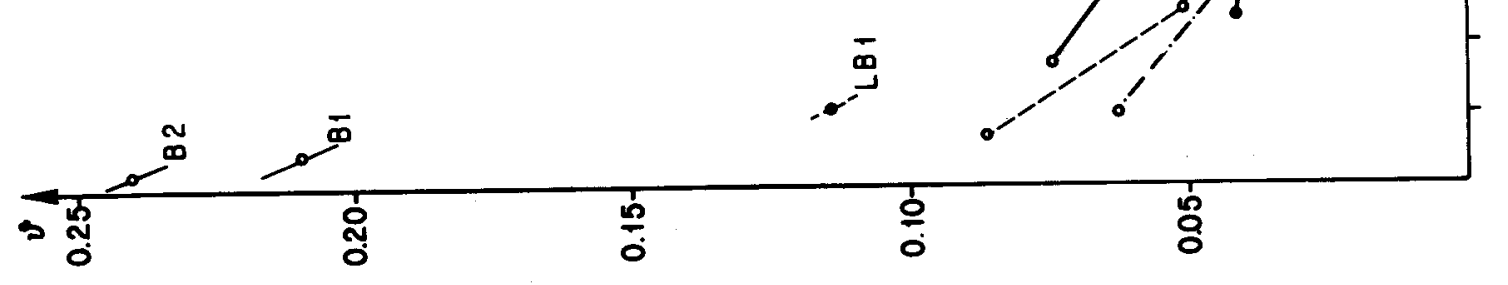

竞-

类
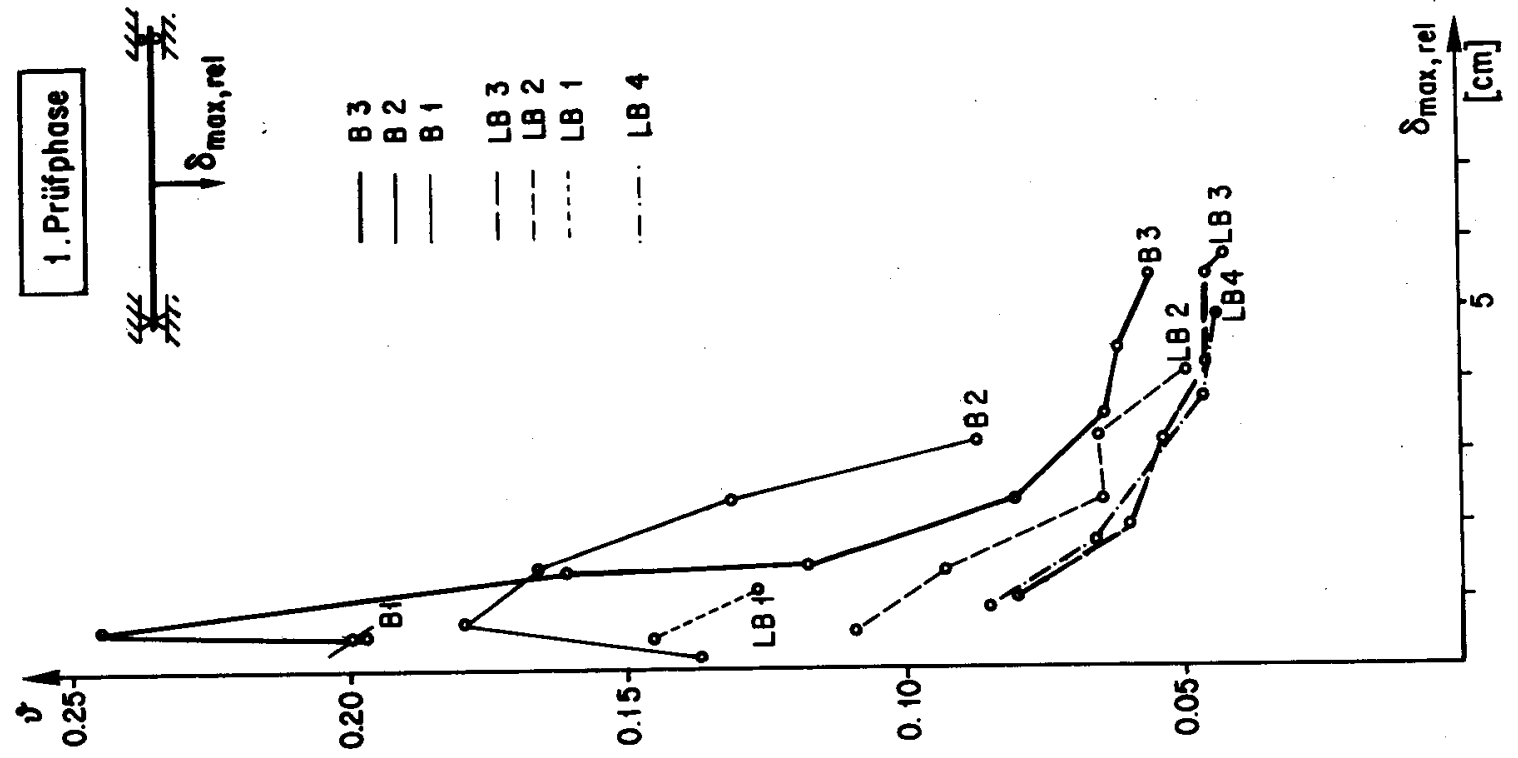

\&

耸 


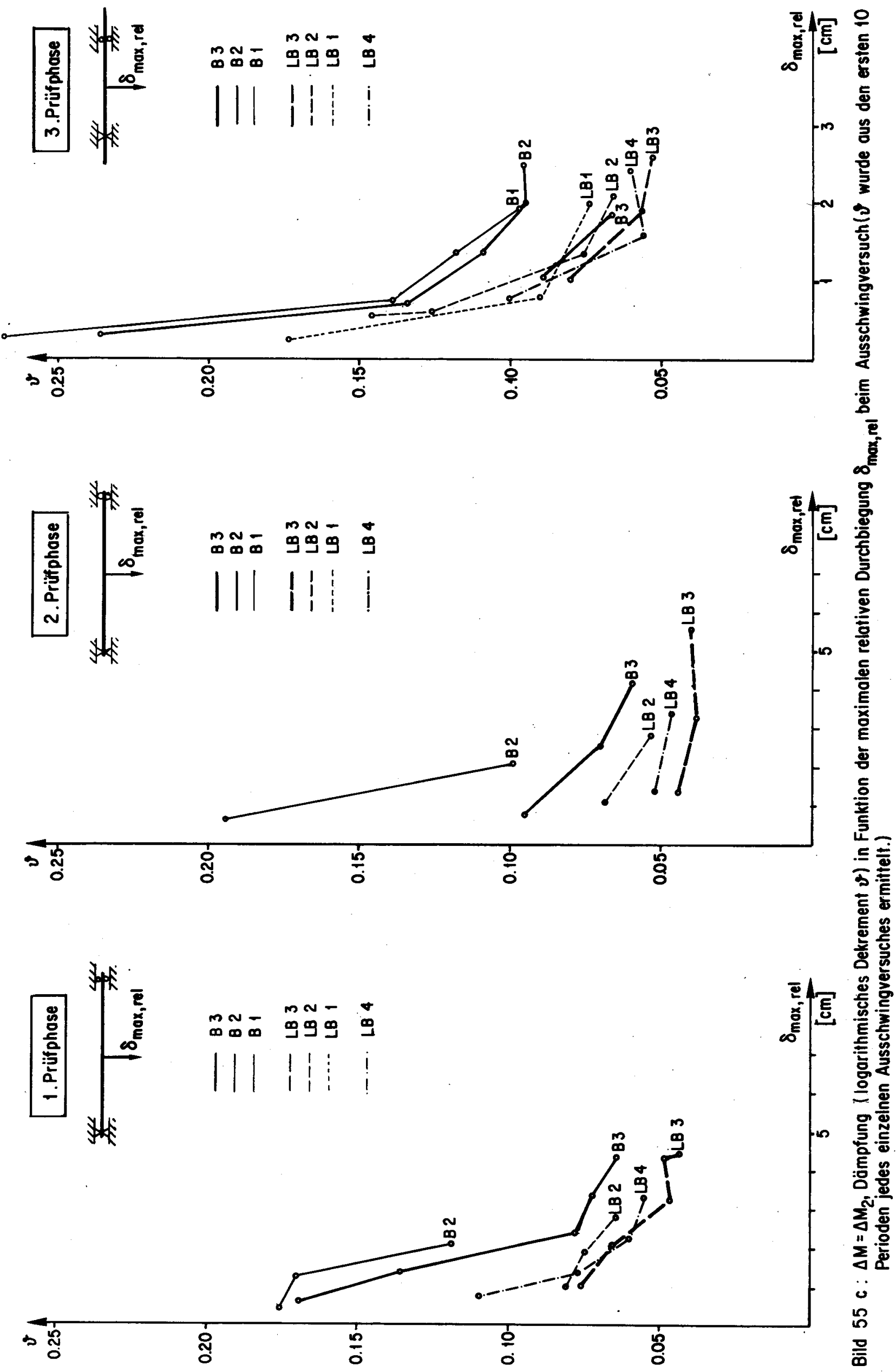




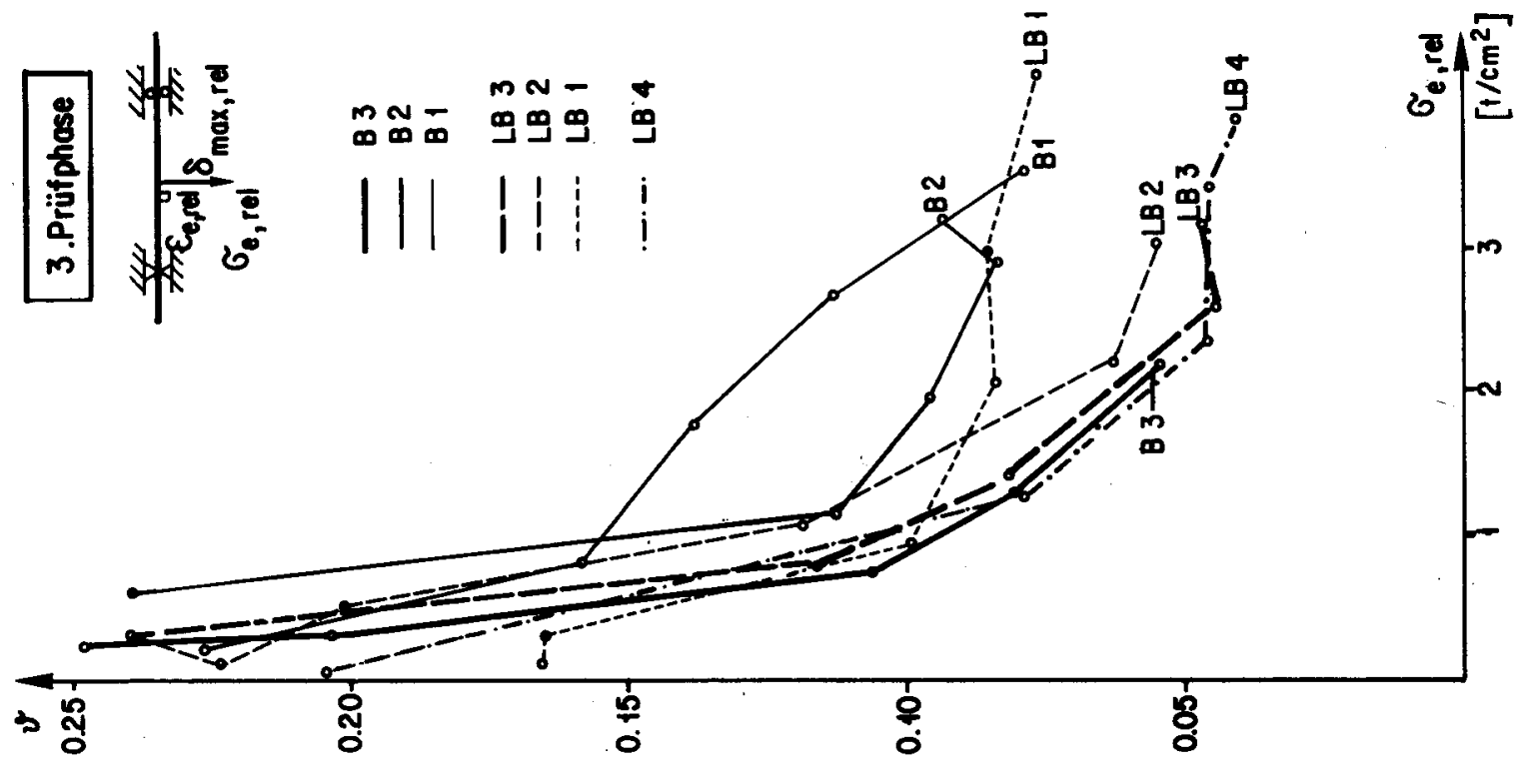

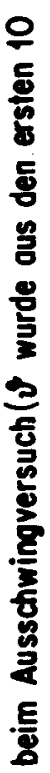

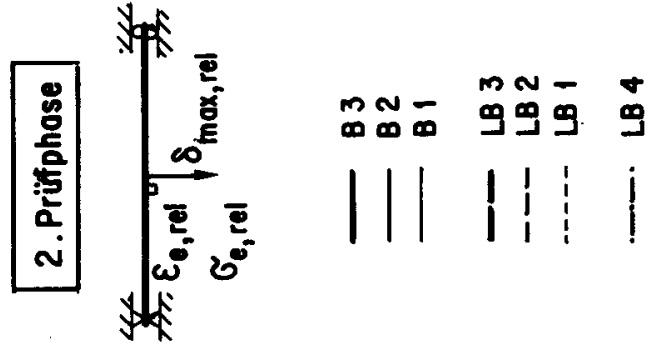




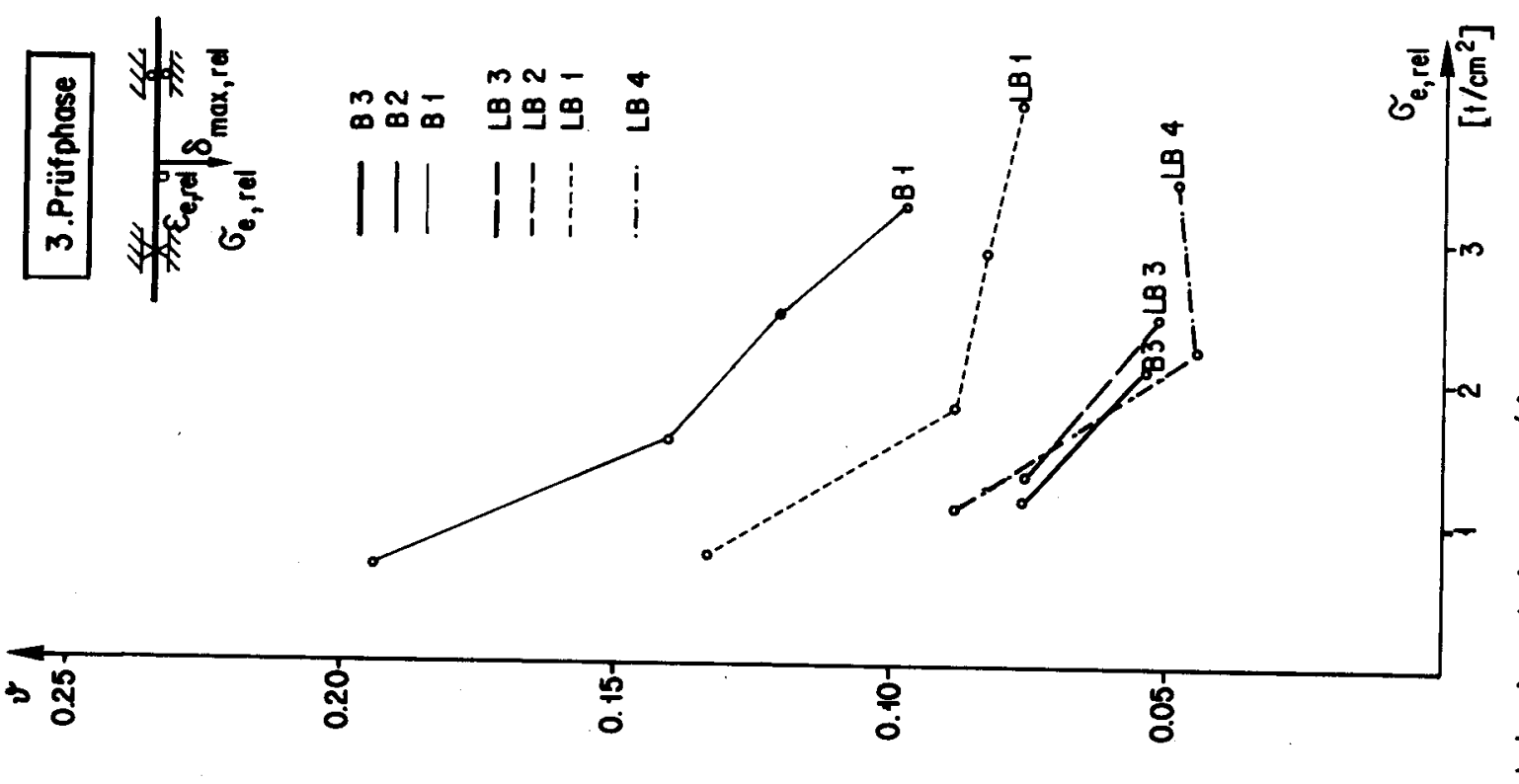

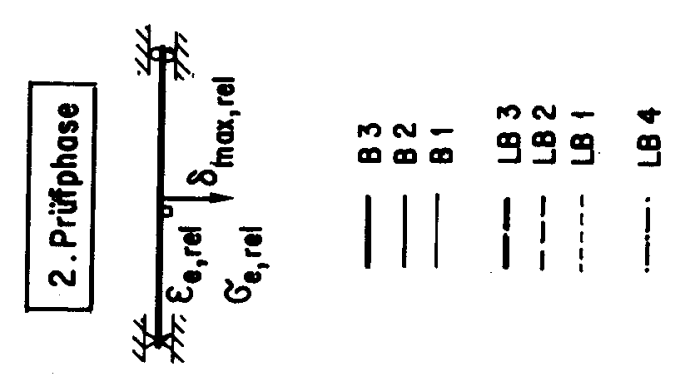
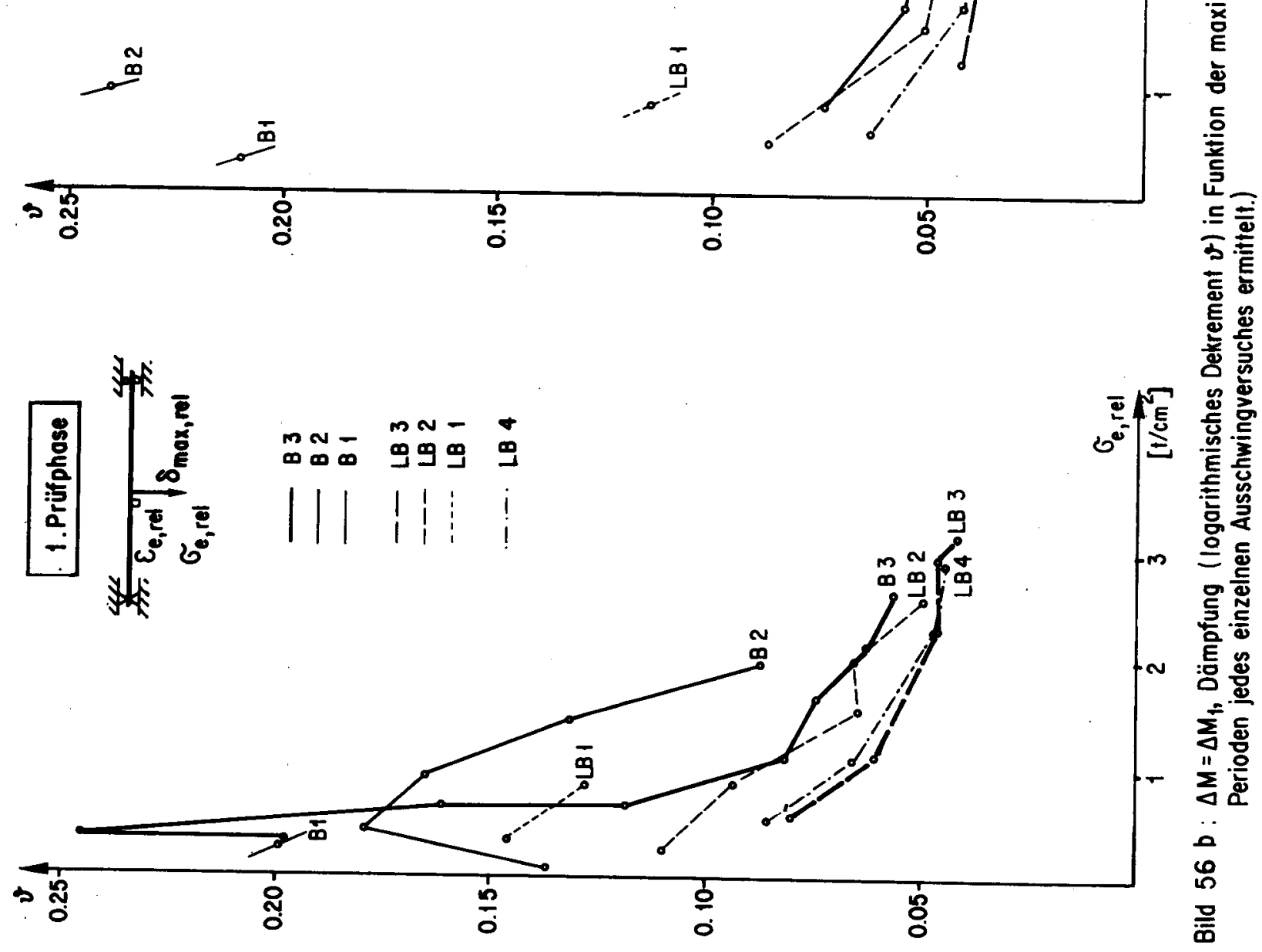


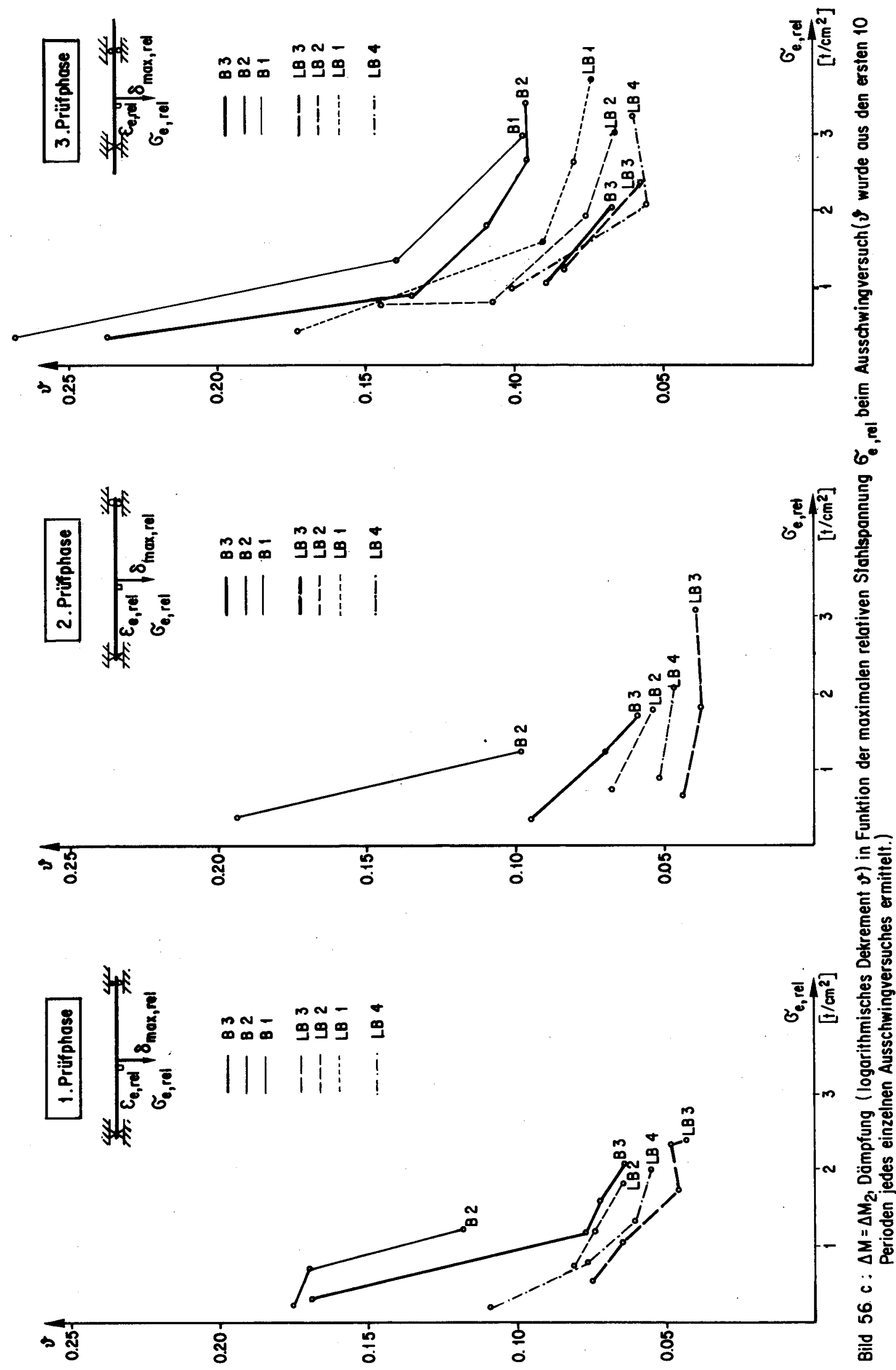




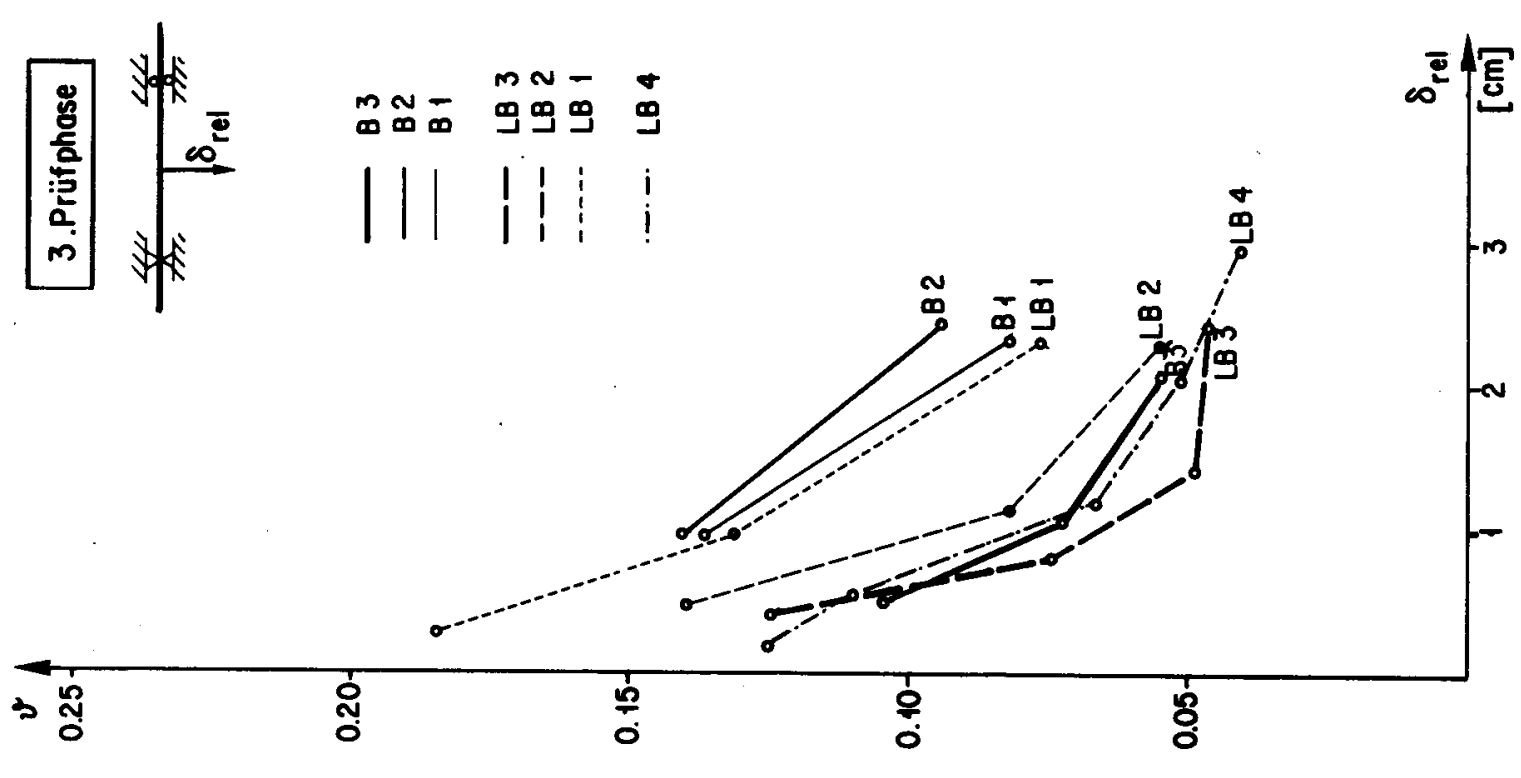


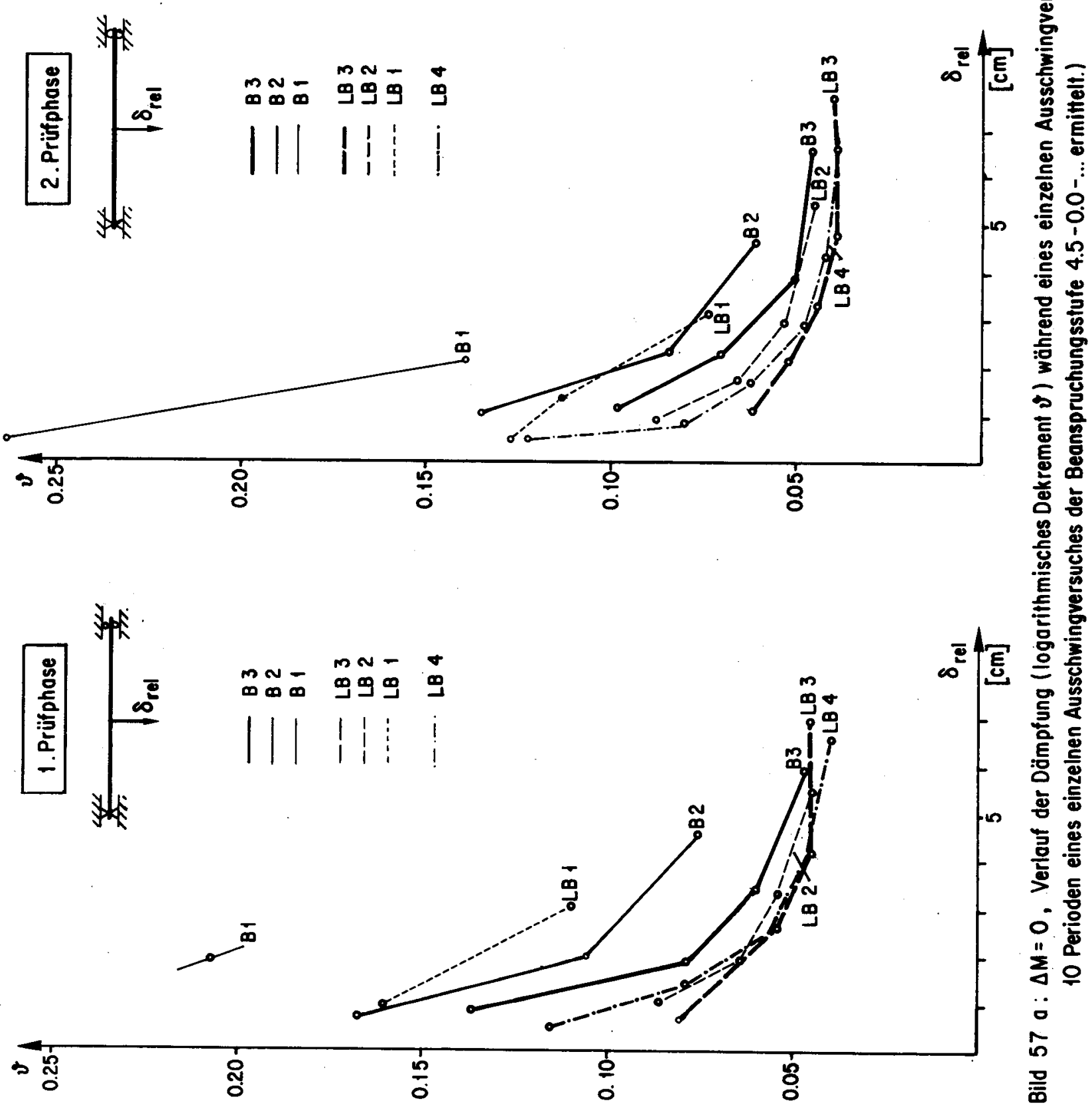

망

ถ

음 

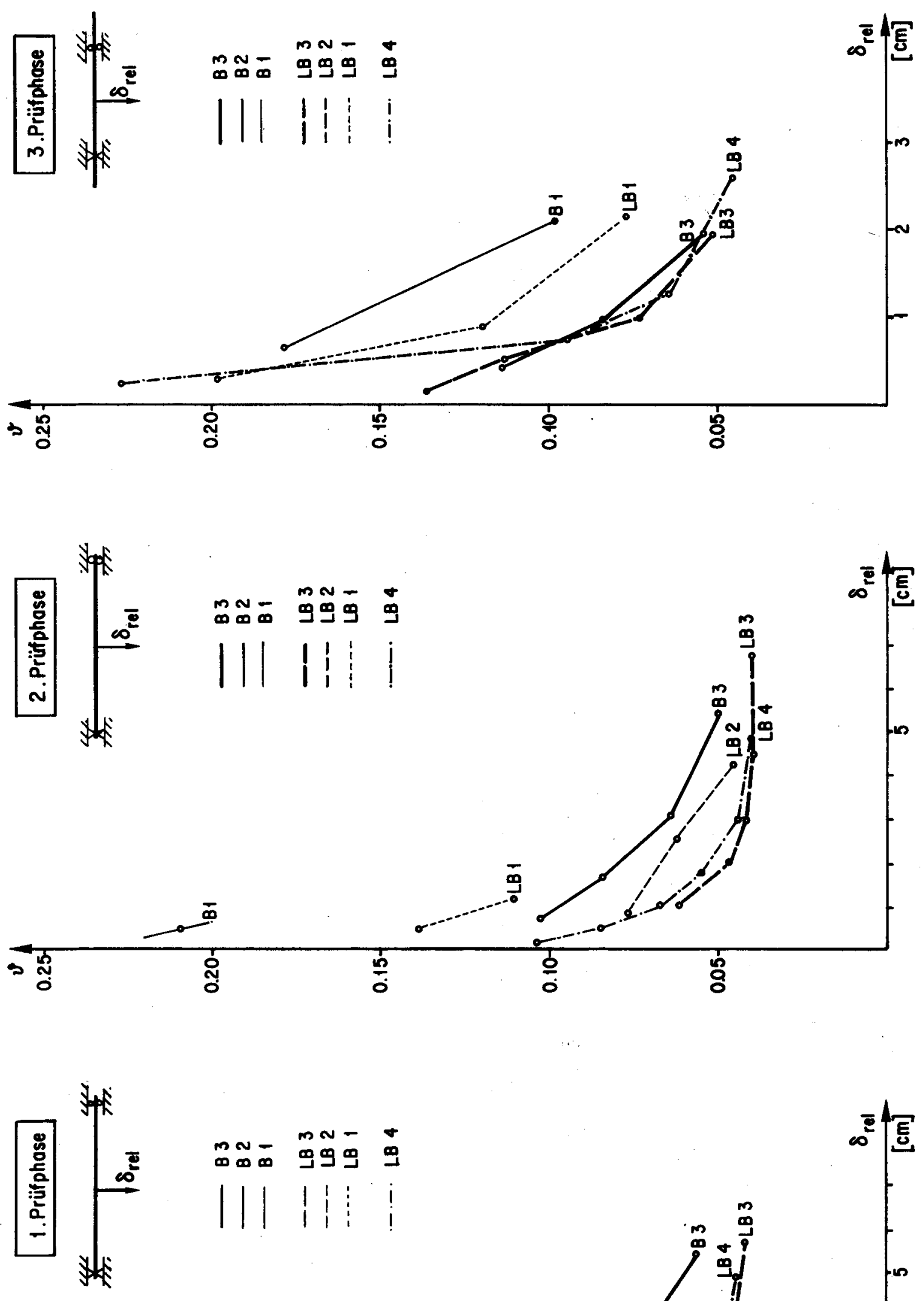

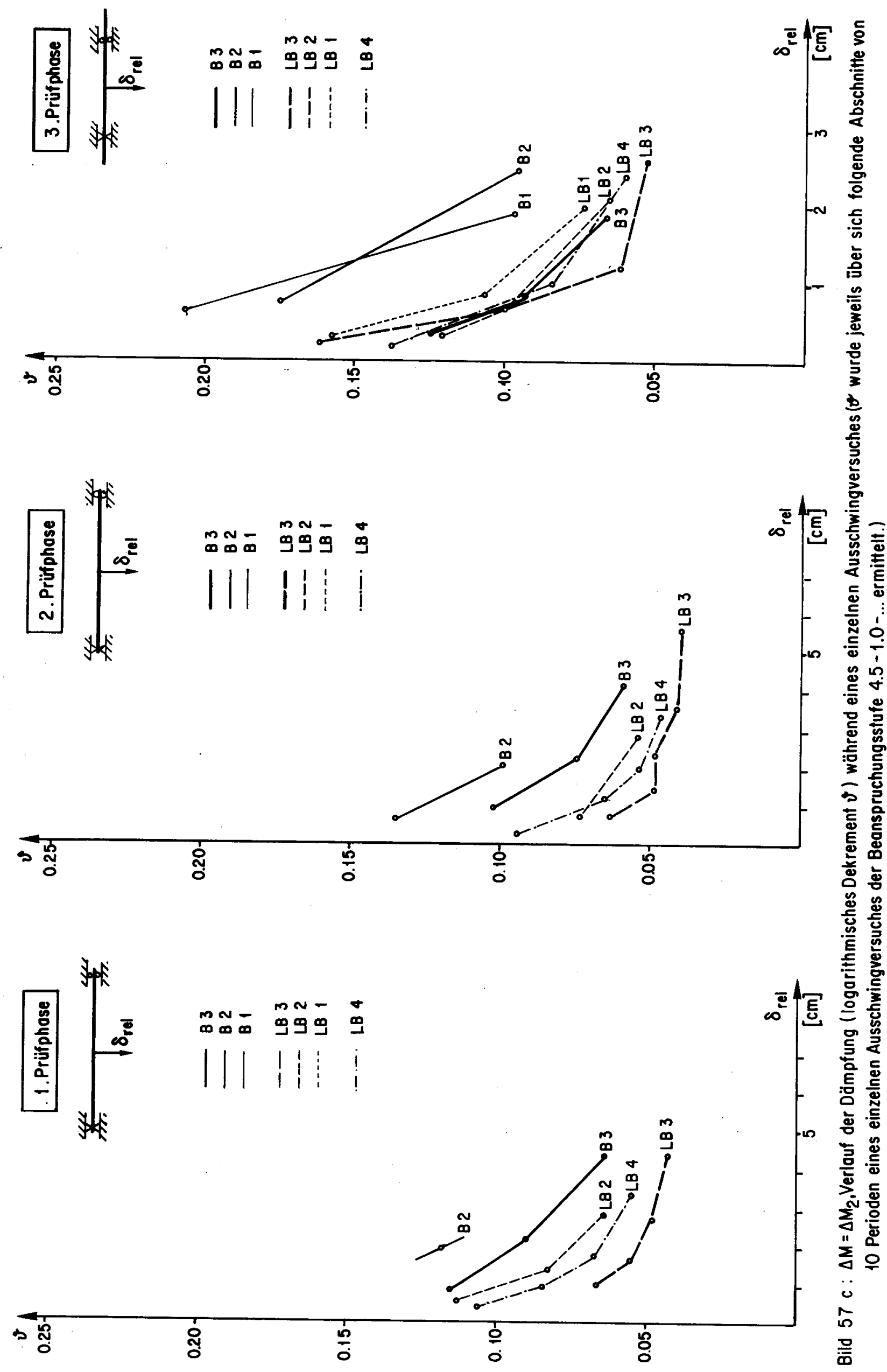

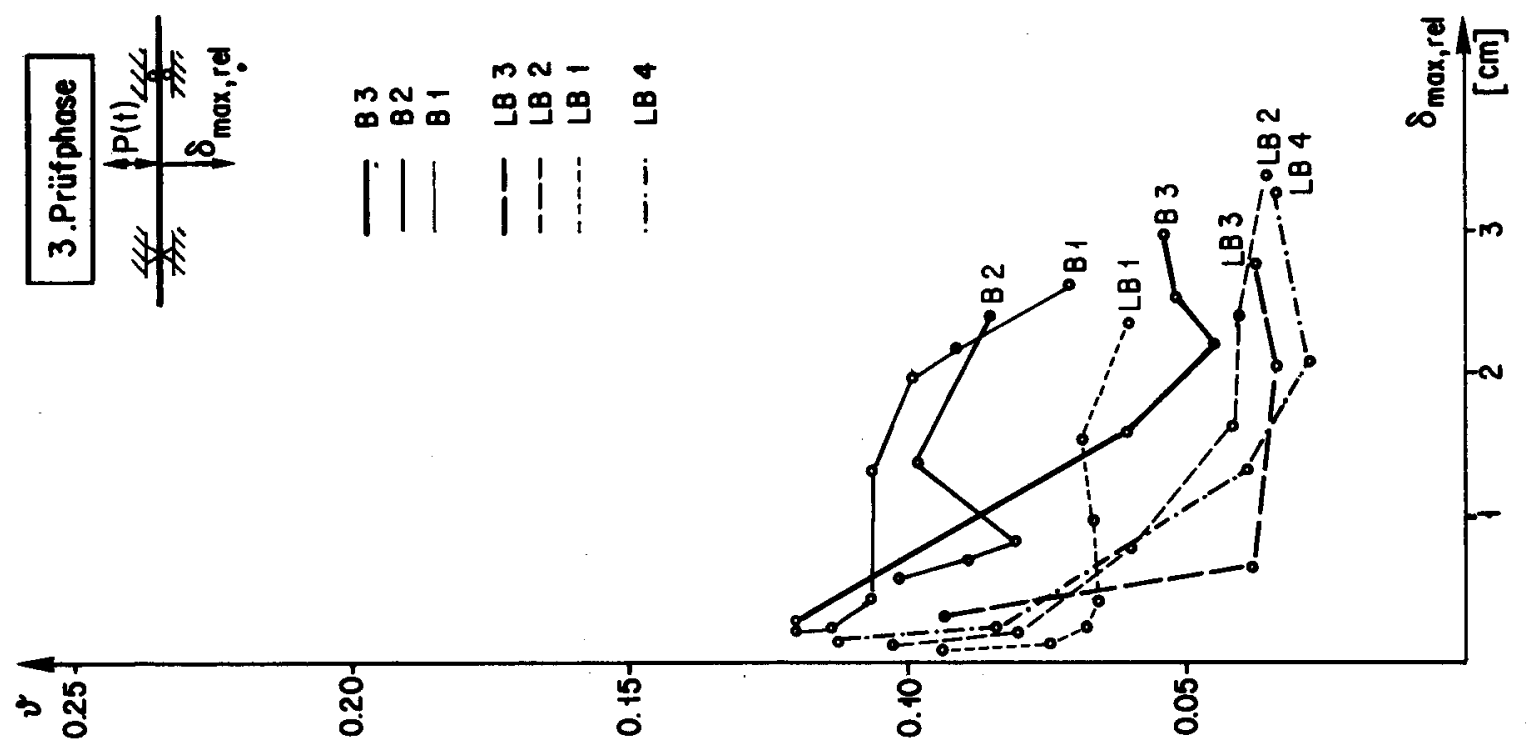

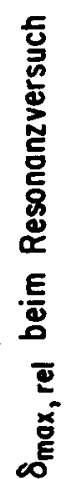
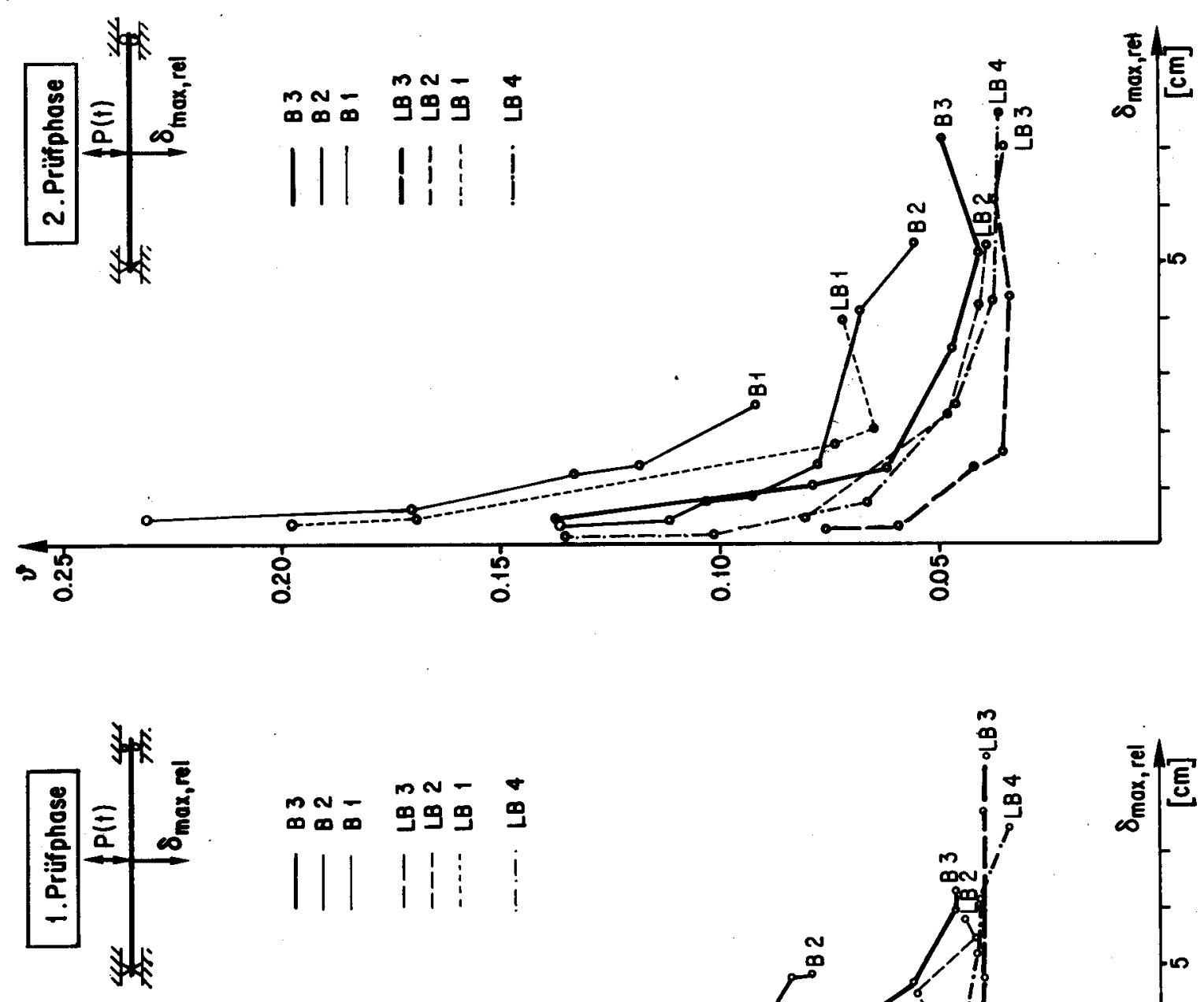


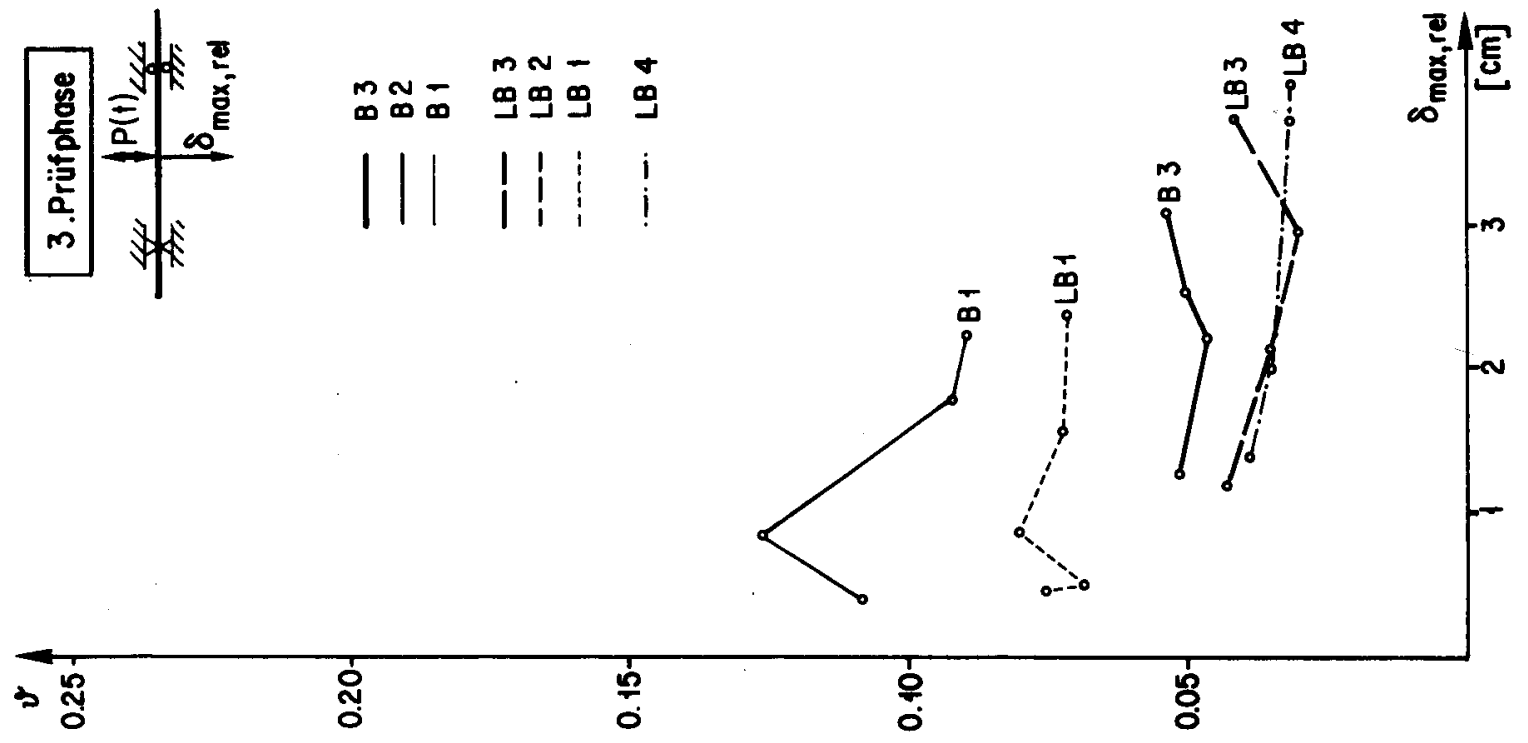

돓
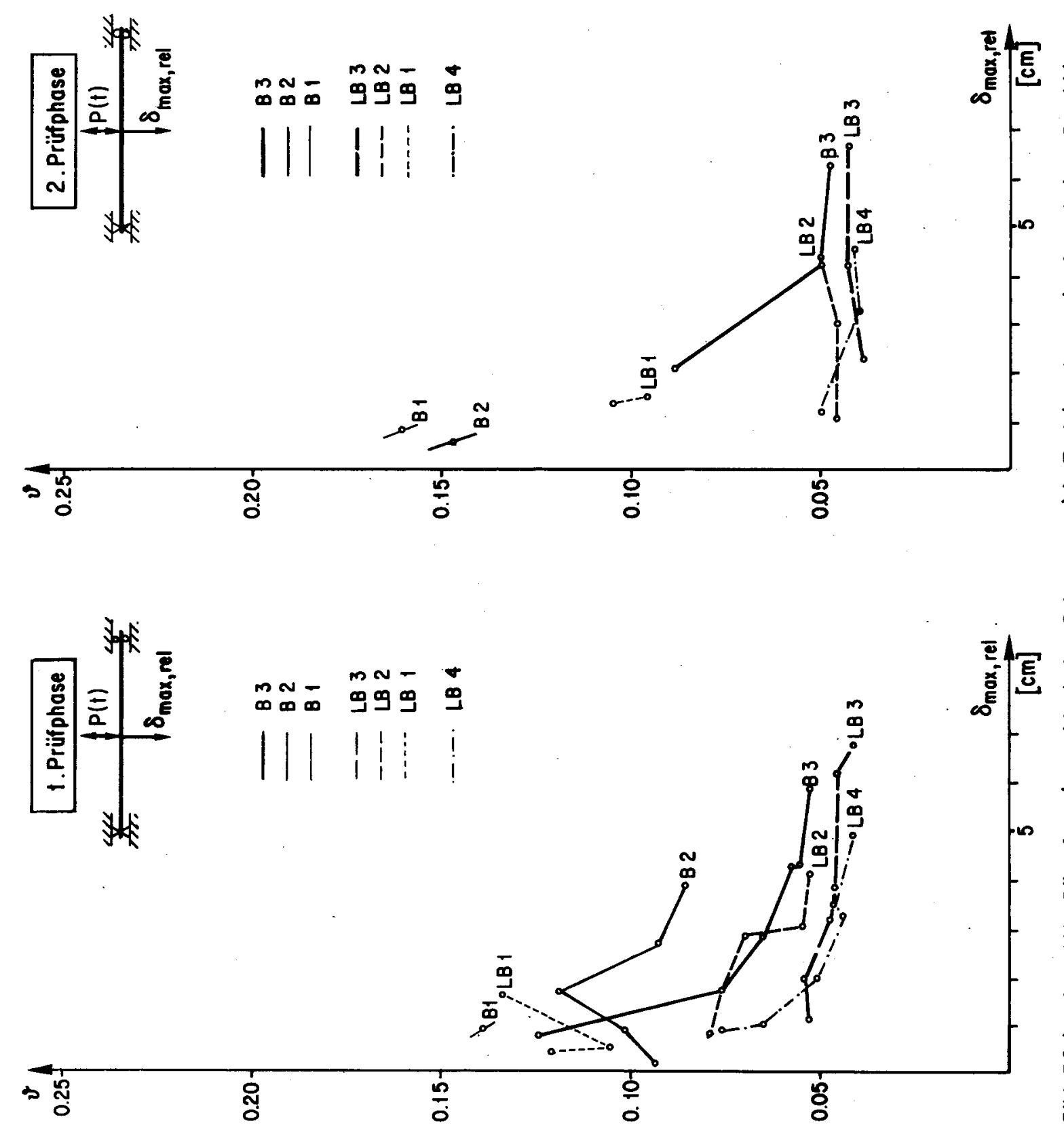

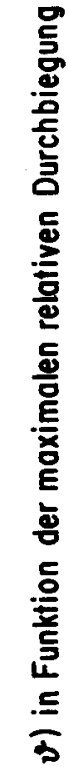

. 

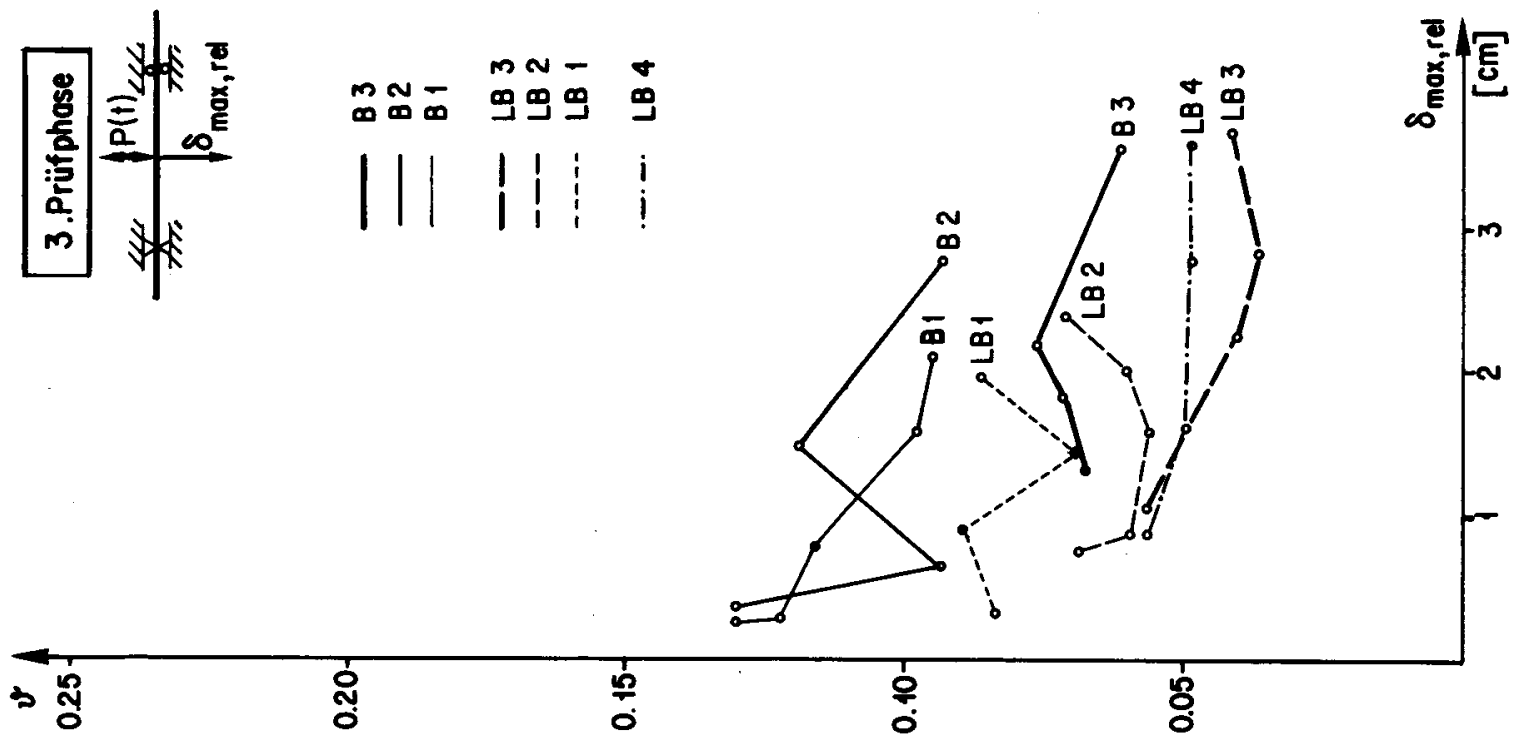

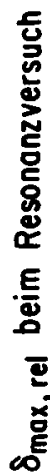
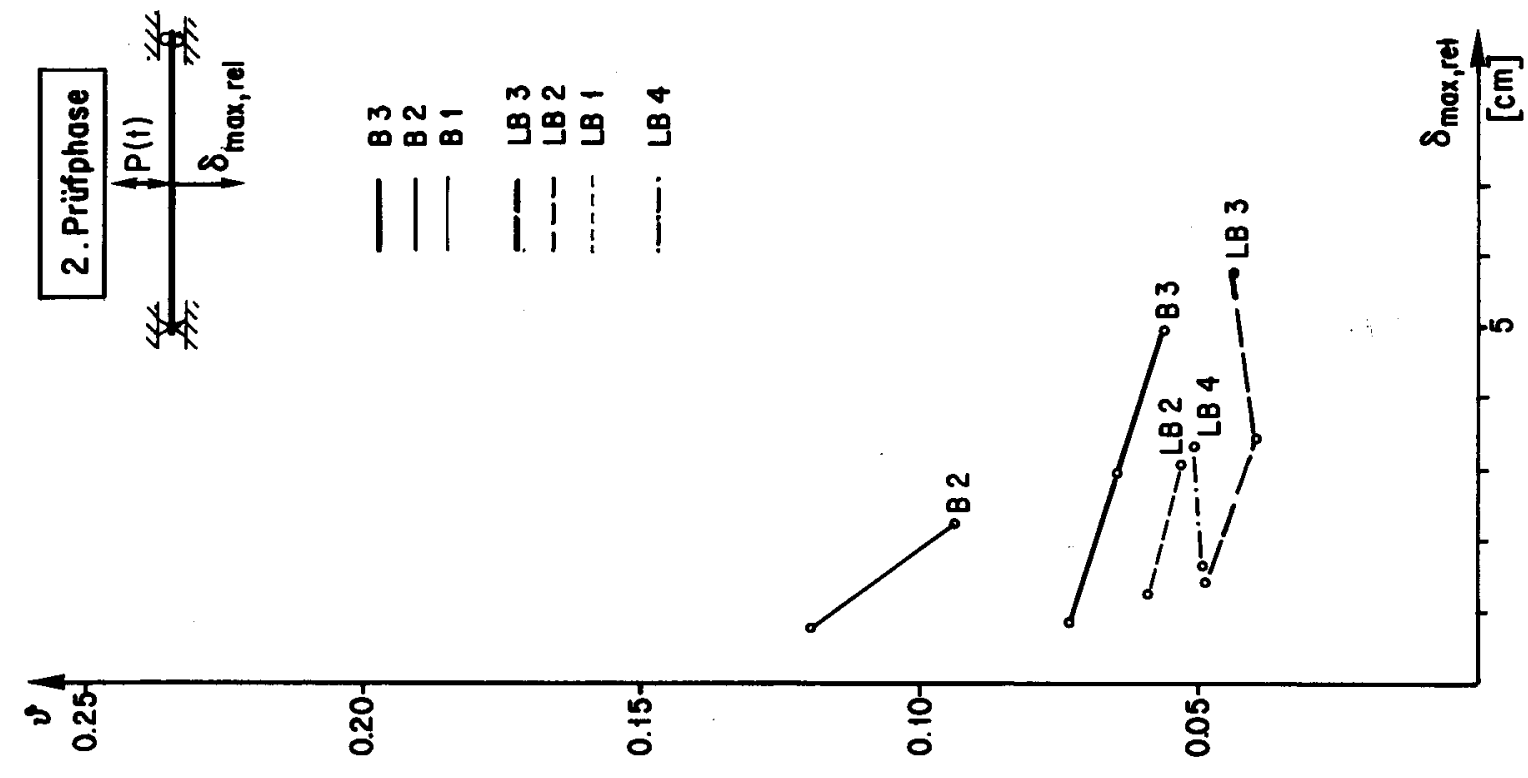

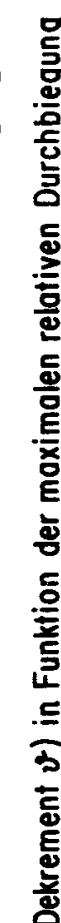
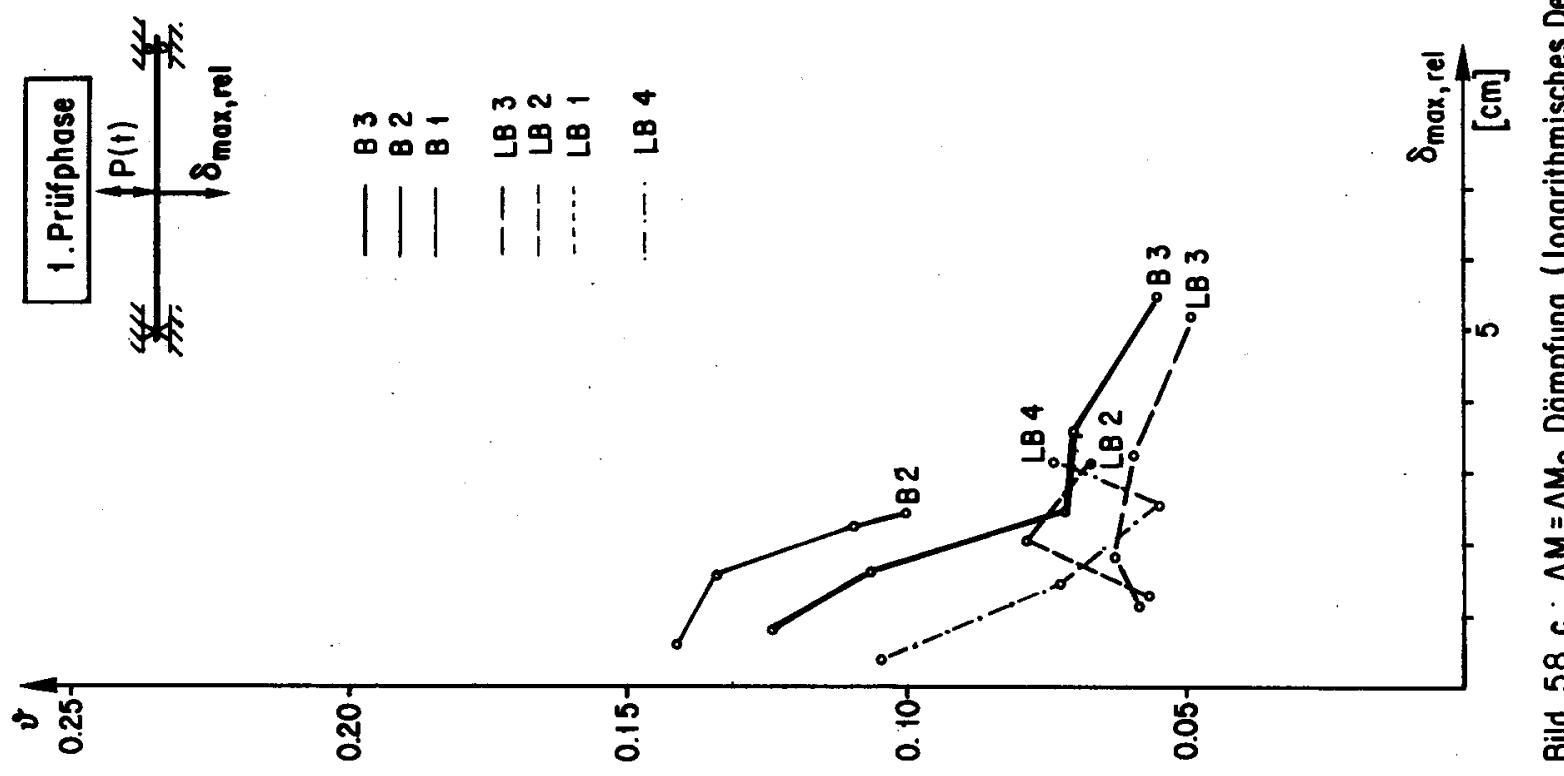


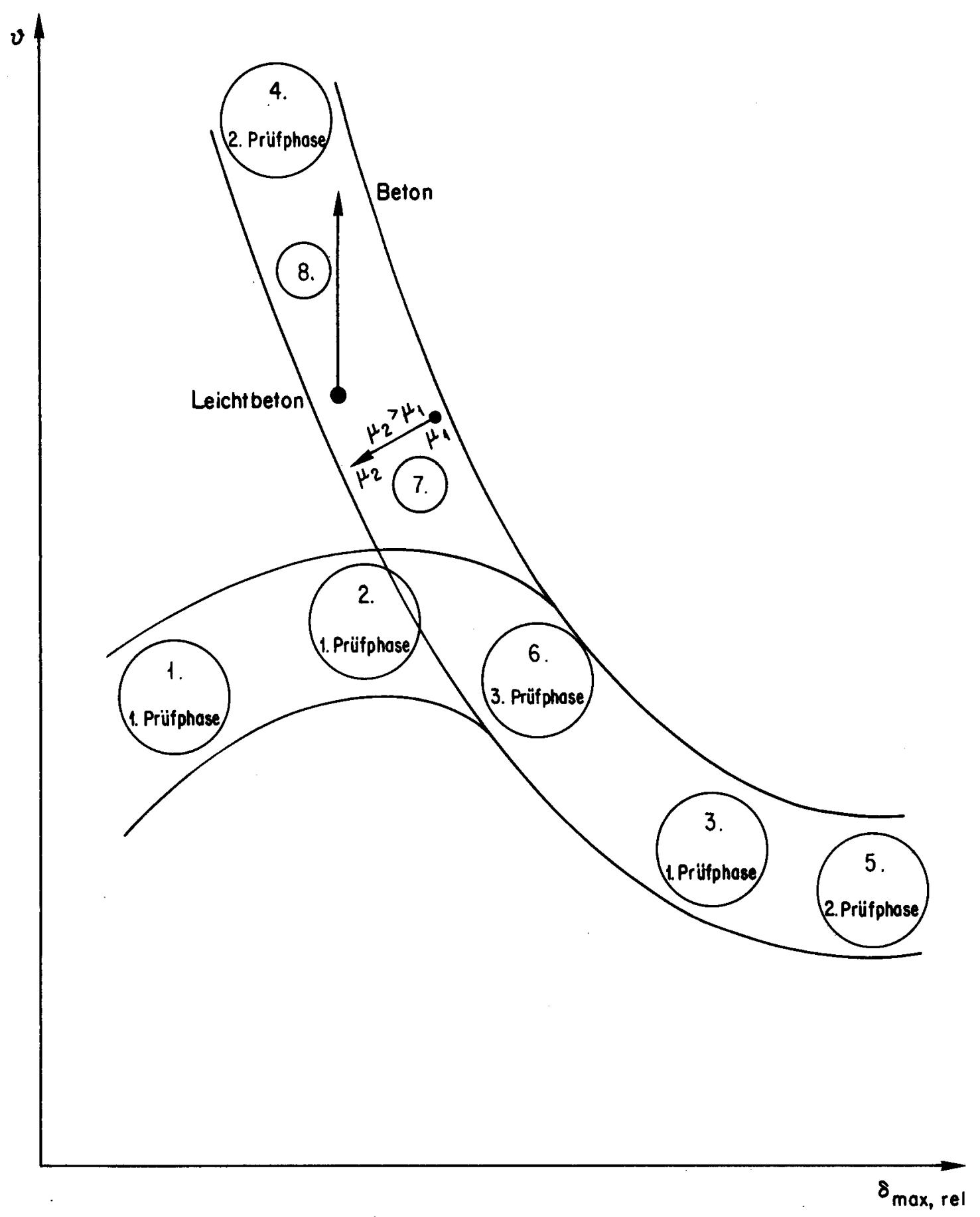

Bild 59: Dämpfung (logarithmisches Dekrement) in Funktion der maximalen relativen Durchbiegung (schematisch) 
Für die beschriebenen Versuche mussten neuartige Belastungs- und Messeinrichtungen entwickelt werden. Im Gegensatz zu den bisher gebräuchlichen Unwuchterregern erlaubt ein elektronisch gesteuerter servohydraulischer Schwingungserreger in einem weiten, baudynamisch interessanten Frequenzbereich innerhalb bestimmter Grenzen beliebige Kräfte auf einen Prüfkörper auszuüben. Dabei sind nebst sinusförmiger Belastung auch andere Zeitfunktionen möglich. Zur Registrierung von Stahl- und Betondehnungen dienen speziell entwickeltè Messgrössenaufnehmer, die sowohl die Aufzeichnungen des ganzen zeitlichen Ablaufs als auch von Spitzenwerten allein ermöglichen.

Servohydraulischer Schwingungserreger (siehe Bilder 24,60 und 61)

An den Schwingungserreger waren folgende Anforderungen zu stellen:

1. Unabhängige Steuerung von Erregerkraft und Erregerfrequenz.

2. Stufenlose Einstellung der Erregerkraft zwischen 10 und $10 \cdot 000 \mathrm{~N}$ und der Erregerfrequenz zwischen 1 und $25 \mathrm{~Hz}$ (Leistungsspektrum).

3. Geringe Eigenmasse.

4. Keine Behinderung der Schwingungen des Prüfkörpers, d.h. keine Abstützung gegen einen ruhenden Rahmen.

Vorerst wurden verschiedene in Hochschulinstituten, Prüfanstalten und Industrie vorhandene Schwingungserreger auf ihre Tauglichkeit geprüft:

- Bei den klassischen Unwuchtschwingern kann die Variation der Erregerkraft nur durch eine Verschiebung der Schwingmasse auf dem Schwungrad erreicht werden. Diese mechanische Einstellung ist umständlich und ungenau. Im übrigen führt eine Aenderung der Erregerfrequenz stets zwingend zu einer Aenderung der Erregerkraft (1. Anforderung nicht erfüllt).

- Elektrodynamische Schwingungserreger erlauben nur kleine Amplituden der Schwingmasse. Sie erzeugen bei den angestrebten Erregerfrequenzen zu kleine Erregerkräfte (2. Anforderung nicht erfülit).

- Ein in der Industrie verwendeter servohydraulischer Schwingungserreger weist mangels Veränderbarkeit der Schwingmasse ein zu geringes Kraft-Frequenz-Leistungsspektrum auf (2. Anforderung nicht erfüllt).

Da in den neuen Forschungsanlagen der Abteilung für Bauingenieurwesen an der ETH-Hönggerberg eine leistungsfähige älhydraulische Pumpenanlage zur Verfügung steht, wurde beschlossen, einen servohydraulischen Schwingungserreger mit dem gewünschten Leistungsspektrum selbst zu entwickeln.

\section{Funktionsprinzip}

Bild 24 zeigt den Schwingungserreger des Institutes für Baustatik und Konstruktion. Das Prinzip ist einfach: In einem Rahmen wird mittels eines hydraulischen Zylinders eine Schwingmasse hin und her bewegt.

Die Delzufuhr zum Zylinder wird durch ein elektronisch gesteuertes Servoventil derart 
geregelt, dass sich die Masse, die mit der Kolbenstange verbunden ist, entsprechend bewegt. Die nötige Delmenge fördert ein Pumpenaggregat. Der Schwingungserreger wird mit dem Prüfkörper fest verbunden. Die auf den Prüfkörper ausgeübten dynamischen kräfte entsprechen im wesentlichen den durch die Hin- und Herbewegung der Schwingmasse $\bar{m}$ entstehenden Trägheitskräften $m \cdot a ̈ ~(a=$ Weg der Masse = Kolbenweg).

\section{Mechanische Teile}

Der Rahmen hat die Aufgabe, die Schwingmasse im Frequenzbereich von 1 bis $25 \mathrm{~Hz}$ und im Wegbereich von 0 bis $10 \mathrm{~cm}$ spielfrei zu führen. Es dürfen keine inneren Resonanzschwingungen auftreten. Schwingungen, die aus Bewegungen des Prüfkörpers senkrecht zur Rahmenebene entstehen, müssen unbeschadet ertragen werden können. Diese Anforderungen werden erfüllt durch eine verhältnismässig steife Ausbildung des Rahmens und eine statisch unbestimmte Lagerung der Verbindungsstange Masse-Zylinder mittels Längskugellagern.

Die Schwingmasse aus Blei kann aus verschiedenen tellerförmigen Einzelmassen zu einer Gesamtmasse zwischen 10 und $200 \mathrm{~kg}$ zusammengesetzt werden. Dadurch ist es mäglich, innerhalb der Leistungsgrenzen der Servohydraulik jede beliebige Kraft-Frequenz-Kombination zu erzeugen.

Der hydraulische Zylinder arbeitet mit einem Systemdruck zwischen 0 und 280 bar. Infolge der Haft- und Gleitreibung im Zylinder eignet sich dieser nur für Frequenzen bis zu $25 \mathrm{~Hz}$. Der Kolbenhub beträgt maximal $10 \mathrm{~cm}$.

Das Servoventil mit einer maximalen Leistung von $57 \mathrm{l} / \mathrm{min}$ regelt den 0elfluss in die Zylinderkammern. Es ist direkt beim hydraulischen Zylinder angeordnet. Dadurch werden sekundäre Einflüsse, wie sie z.B. aus Querschnittsänderungen der Rohrleitungen durch die rasch wechselnden Drücke entstehen, weitgehend vermieden.

Das Pumpenaggregat zum Betrieb des Schwingungserregers fördert bei einem Betriebsdruck von 280 bar eine Delmenge von 10,40 oder $50 \mathrm{l} / \mathrm{min}$.

\section{Elektronische Steuerung und Ueberwachung}

Die Steuerung des Schwingungserregers (Bild 60) erfolgt über das Servoventil als Bindeglied zwischen Elektronik und Hydraulik derart, dass Amplitude und Frequenz der Kolbenbewegung den bestimmten gewünschten Grössen entsprechen. Das erforderliche Steuersignal wird im elektronischen Analog-Regler aus der Differenz zwischen Ist- und Sollwert ermittelt. Der Ist-Wert (oder Messwert) lässt sich mit dem Kolbenwegmesser oder mit dem Kraftmesser erfassen. Als Sollwertgeber dient ein Funktionsgenerator.

Der Kolbenwegmesser besteht aus einem induktiven Wegaufnehmer, der in der hohlen Kolbenstange untergebracht ist. Er erzeugt - entsprechend dem Standort der Schwingmasse - ein Spannungssignal.

Der Kraftmesser zwischen Kolbenstange und Schwingmasse ist ein speziell entwickeltes ringförmiges Element. Die Biegedehnungen der beiden Schenkel werden mit Dehnungsmessstreifen in Vollbrückenschaltung erfasst. Die Kraft entspricht dem erzeugten Spannungssignal und ist - abgesehen von vernachlässigbar kleinen elastischen Kräften und Dämpfungskräften - gleich der vom Schwingungserreger auf den Prüfkörper ausgeübten dynamischen Kraft.

Der Funktionsgenerator hat die Aufgabe, ein Spannungssignal entsprechend dem Sollwert zu erzeugen. 
Der Analogregler vergleicht den Ist- mit dem Sollwert und liefert einen zur Differenz der beiden Spannungsignale proportionalen Steuerstrom.

Die Ueberwachung des Schwingungserregers geschieht durch Messung der wirksamen dynamischen Kraft in der Verbindungsstange Masse-Zylinder sowie durch einen entsprechenden Sicherheitsschaltkreis. Dieser ermäglicht eine akustische oder optische Warnung bzw. ein Abschalten des Schwingungserregers bei Ueberschreiten festgelegter Grenzen der Kraft.

\section{Charakteristische Grössen}

Die dynamische Kraft des Schwingungserzeugers ergibt sich theoretisch für eine sinusförmige Bewegung $=a_{0} \cdot \sin \Omega t$

zu

$P_{\text {Err,max }}=m \cdot a \cdot \Omega^{2}=\frac{G}{g} \cdot a \cdot 4 \pi^{2} \cdot f^{2}$

mit :

$\Omega=K r e i s f r e q u e n z$ der Bewegung $\left(\sec ^{-1}\right)$

$f=$ Frequenz der Bewegung $\left(\sec ^{-1}\right)$

$G=$ Gewichtskraft $(k N)$

$g=$ Erdbeschleunigung $\left(m \cdot \sec ^{-2}\right)$

Durch verschiedene Einflüsse wie Charakteristik des Servoventils, Reibung zwischen Kolben und Zylinder, Lagerreibung, usw. ergeben sich indessen gewisse Abweichungen. Die Leistungswerte des Schwingungserregers sind ferner durch die Kapazitäten von Pumpenaggregat, Servoventil und Hydraulikzylinder begrenzt. Bild 61 zeigt als Beispiel die im Prüfstand gemessene tatsächliche Kraft-Frequenz-Grenzkurve des Schwingungserregers für eine Masse von $104 \mathrm{~kg}$. Zum Vergleich ist die theoretische, aufgrund der Leistungsfähigkeiten von Hydraulik und Elektronik ermittelte Kurve gestrichelt eingetragen. Unterhalb der gemessenen Grenzkurve kann jede beliebige Kraft-Frequenz-Kombination realisiert werden.

\section{Messeinrichtungen (siehe Bilder 62 und 63)}

Analoge Aufzeichnung und Spitzenwertmessung:

Messungen bei dynamischen Beanspruchungen bedingen eine umfangreiche Ausrüstung, sofern jeder Messstelle während des ganzen Versuchsablaufs eine komplette Registriereinheit (Kanal) zur analogen Aufzeichnung zugeordnet werden muss. Wenn jedoch die zeitliche Funktion der Bewegung als solche stationär und zudem bekannt ist, kann der Aufwand mittels einer Umschaltanlage und nachgeschalteter Spitzenwertmessung stark verringert werden. Die am Institut für Baustatik und Konstruktion zur Verfügung stehende Anlage erlaubt 100 Messpunkte seriell nach dem oberen und unteren Spitzenwert abzutasten. Diese Messart erfordert für jede Messstelle nur den fest verdrahteten Messwertgeber. Die entsprechenden Messwerte werden dann chronologisch durch eine einzige Gerätegruppe registriert. Das Abtasten und Registrieren der Spitzenwerte von 100 Messstellen dauert etwa 100 sec.

\section{Dreigelenk-Dehnungsmessrahmen:}

Die Deformationsmessung beim Beton schliesst zum vorneherein den direkt applizierten Dehnungsmessstreifen aus, da dieser durch Rissbildung Schaden nimmt und infolge von Inhomogenitäten der Betonoberfläche oft zu Fehlmessungen führt. Induktive Wegaufnehmer sind nur geeignet, wenn die hohen Beschaffungskosten und der beträchtliche Montageaufwand keine Rolle spielen. Aus diesen und weiteren Gründen musste für die erwähnten Balkenversuche eine neuartige Messeinrichtung gesucht werden.

Die Aufgabe bestand darin, über einer Basislänge von $20 \mathrm{~cm}$ die Dehnung auf dem Versuchsobjekt mit einer Genauigkeit von etwa $1 \%$ zu erfassen. Die Lösung wurde im sog. Dreigelenk- 
Dehnungsmessrahmen gefunden (Bild 62). Das mittlere Gelenk, eine $2.85 \mathrm{~cm}$ lange Leichtmetall-Lamelle, dient als eigentliches Messelement. Die Fusspunkte des Dehnungsmessrahmens sind als wirkliche Gelenke mit konischen Axen zum spielfreien Einstellen ausgebildet. Die Fixierung des Gerätes auf dem Beton oder Bewehrungsstahl des Versuchsobjektes erfolgt mit normalem Dehnungsmessstreifen-Klebstoff.

Ụm eine vollständige Endeinspannung der Lamelle zu erhalten, wurde sie aus dem vollen Material herausgefräst. Diese Ausbildung bewirkt eine konzentrierte Biegeverformung über die Länge der Lamelle und bildet die Grundlage einer exakten Verformungsmessung durch zwei beidseitig auf die Lamelle geklebte Dehnungsmessstreifen in Halbbrückenschaltung. Bei dieser Schaltung werden die absoluten Dehnungsbeträge der Zug- und Druckfaser der Lamelle addiert. Eine günstige Kombination von Rahmenhöhe, Gelenklänge und Gelenkhöhe führt zu einer kalibrierten Aussage mittels herkömmlicher Messbrücken. Im verwendeten Messgerät kann als kleinste Einheit am manuellen Kompensator bei einer k-Wert-Einstellung von 2.0 noch eine Dehnung von $0.001 \%$, d.h. $10^{-5}$ abgelesen werden. Bei einer direkten Messung über einen Messverstärker lässt sich die Auslösung noch um den Faktor 100 verbessern.

Der geometrische Zusammenhang, der innerhalb der üblichen Verformungen von Betonprüfkörpern die Genauigkeitsanforderungen erfüllt, ist in Bild 63 festgehalten. Weitere beeinflussende Parameter sind K-Faktor der verwendeten Dehnungsmessstreifen und Umschaltgeräte. Die Reibungskraft im Fussgelenk ist so klein, dass sie die Reproduzierbarkeit der Messung nur unmerklich beeinflusst. Die Dreigelenk-Dehnungsmessrahmen können im elastischen Bereich des verwendeten Materials - im vorliegenden Fall bis zu Längenänderungen von $\pm 4 \mathrm{~mm}$ - verwendet werden. Selbst bei Schwingungen von $25 \mathrm{~Hz}$ sind noch exakte Aussagen über das tatsächliche Dehnungsverhalten möglich. 


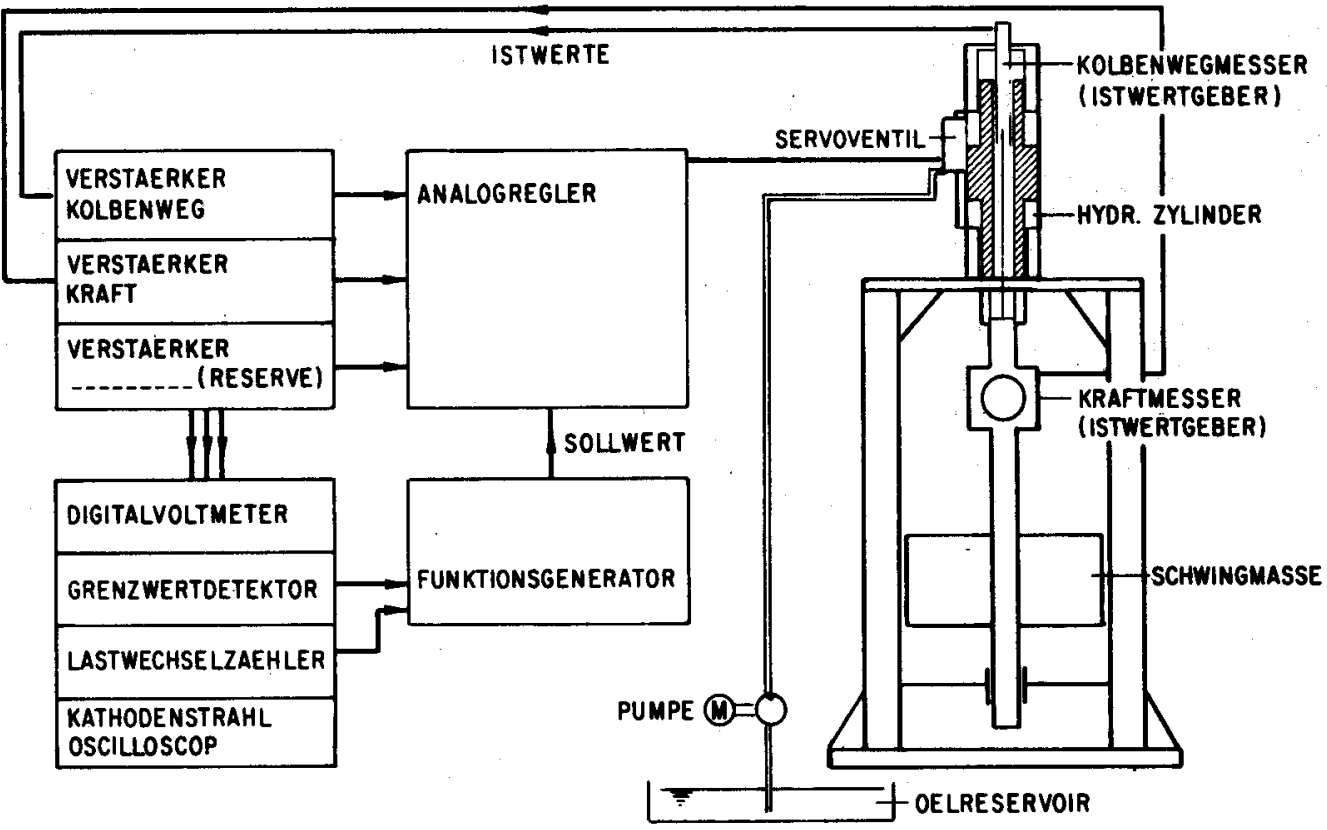

Bild 60: Schemo der Steuerung (Regelkreis)

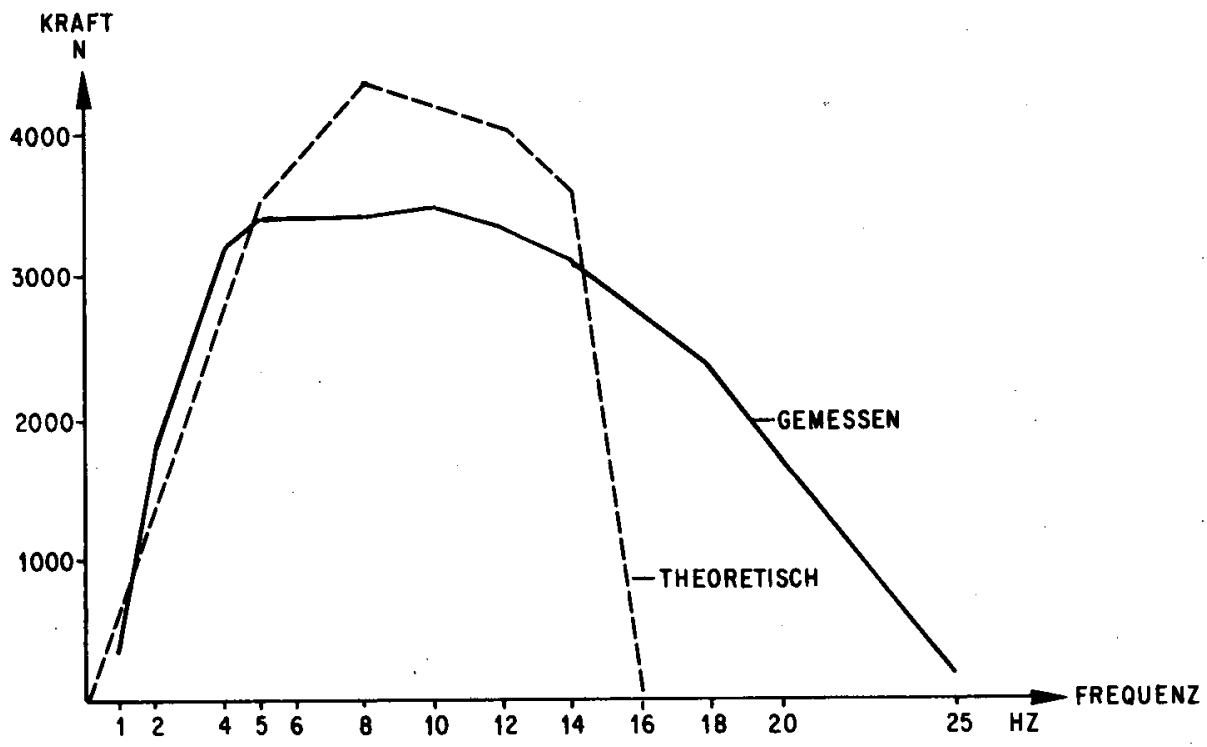

Bild 61: Kraft -Frequenz-Grenzkurve des Schwingungserregers für eine Masse von $104 \mathrm{~kg}$ 


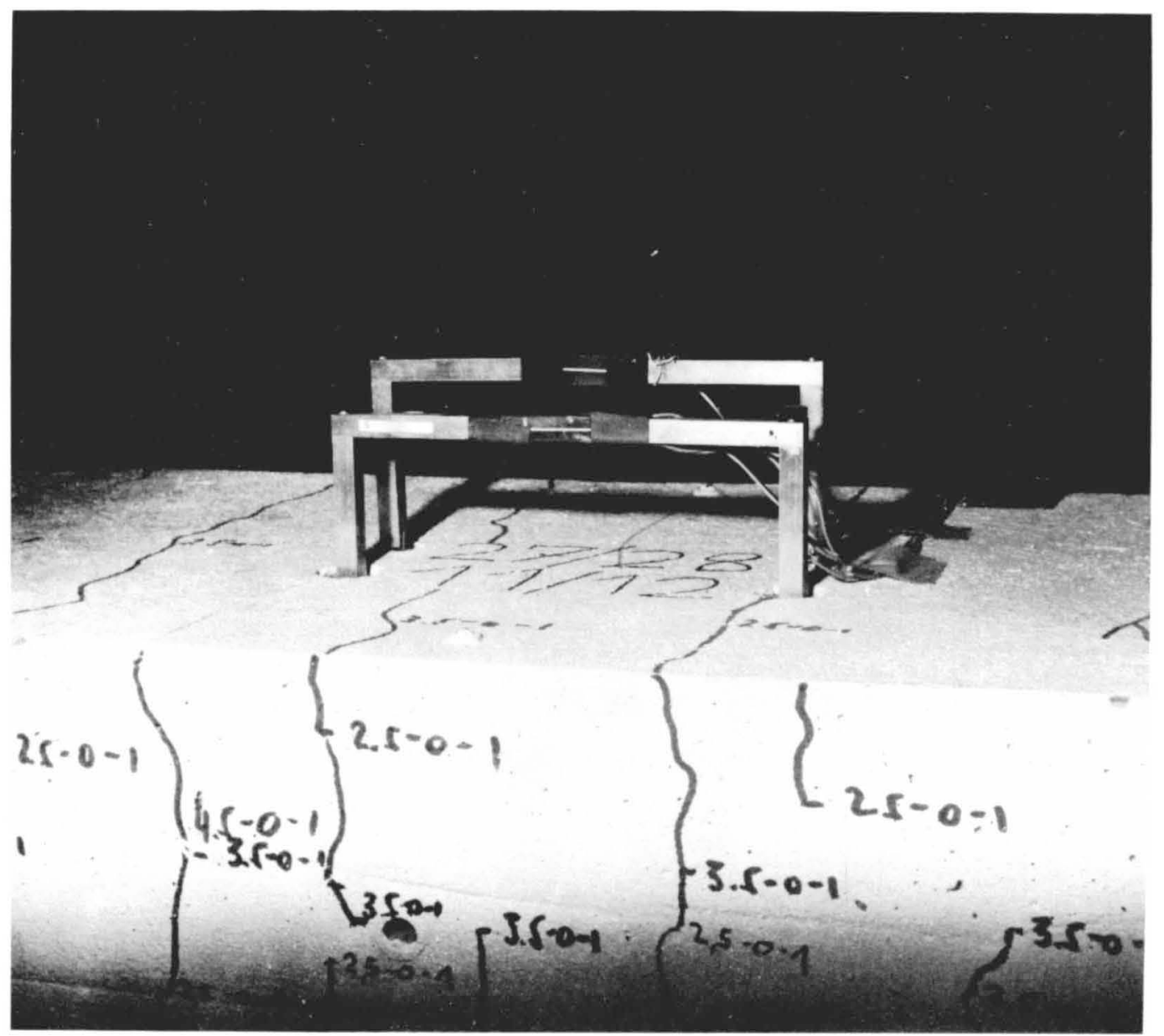

Bild 62: Dreigelenk-Dehnungsmessrahmen

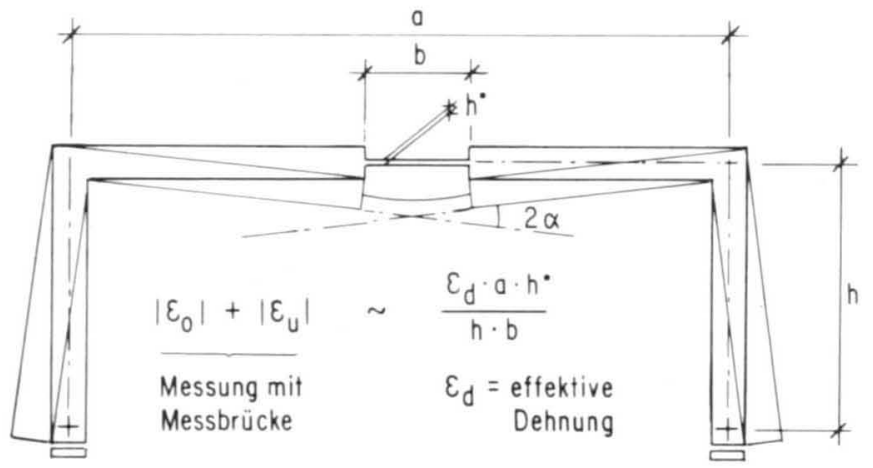

Bild 63: Geometrie des Dreigelenk-Dehnungsmessrahmens 